\title{
Aufbau einer HPLC-UV-ESI-MS/MS-Datenbank und ihre Anwendung im Screening arktischer und antarktischer Meeresbakterien
}

\author{
Dissertation \\ zur Erlangung des Doktorgrades \\ der Mathematisch-Naturwissenschaftlichen Fakultäten \\ der Georg-August-Universität zu Göttingen
}

\author{
vorgelegt von \\ Imelda Schuhmann \\ aus Surabaya, Indonesien
}

Göttingen 2005 
D7

Referent: Prof. Dr. H. Laatsch

Korreferent: Prof. Dr. A. Zeeck

Tag der mündlichen Prüfung: 29. Juni 2005 
Die vorliegende Arbeit wurde im Institut für Organische und Biomolekulare Chemie der Universität Göttingen in der Zeit von Juni 2002 bis Mai 2005 unter Anleitung von Prof. Dr. H. Laatsch durchgeführt.

Herrn Prof. Dr. H. Laatsch danke ich für die interessante Aufgabenstellung, sein stetes Interesse am Fortgang dieser Arbeit sowie für viele wertvolle Diskussionen und Anregungen. 


\section{Inhaltsverzeichnis}

1 Einleitung ................................................................................................................. 1

$1.1 \quad$ Naturstoffe aus marinen Organismen............................................................

1.2 Arktische und antarktische Meereisbakterien ................................................ 4

1.3 Symbiotische und pathogene Mikroorganismen .............................................. 6

2 Aufgabenstellung................................................................................................. 9

$3 \quad$ Screening der Bakterienstämme.............................................................................. 11

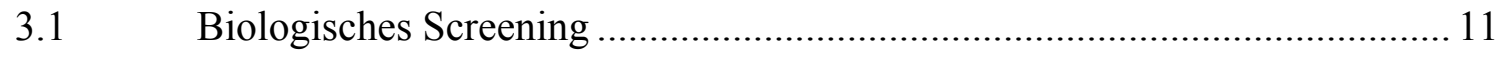

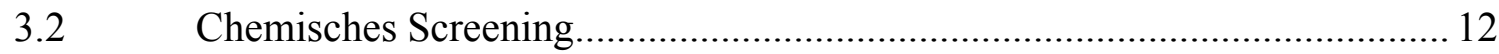

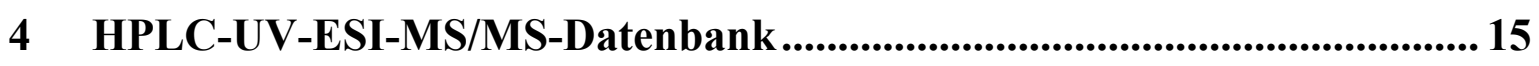

4.1 Aufbau einer HPLC-UV-ESI-MS/MS-Datenbank......................................... 15

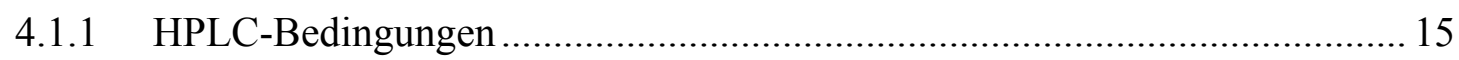

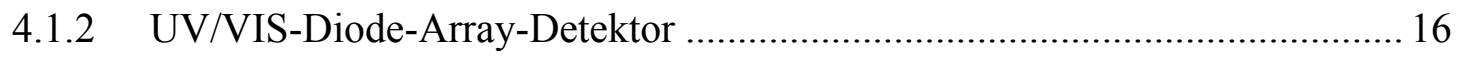

4.1.3 Electrospray-Massenspektrometrie ……………..................................... 16

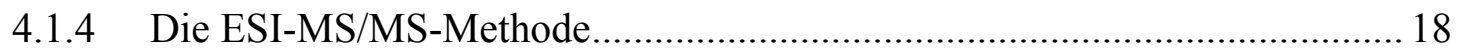

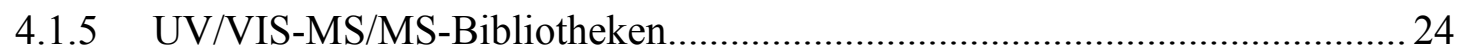

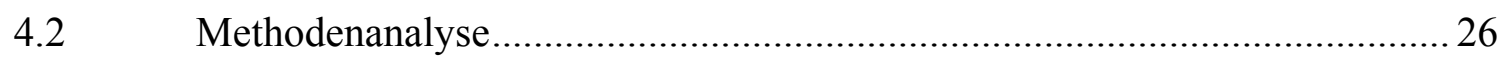

5 Fragmentierungen ausgewählter Substanzen .....................................................30

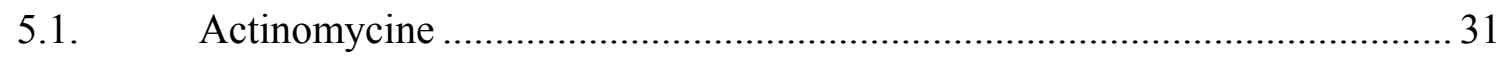

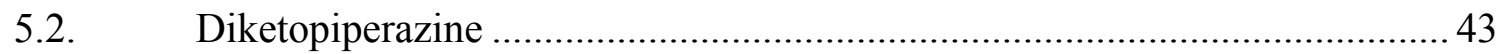

6 Untersuchung ausgewählter Stämme aus arktischen und antarktischen

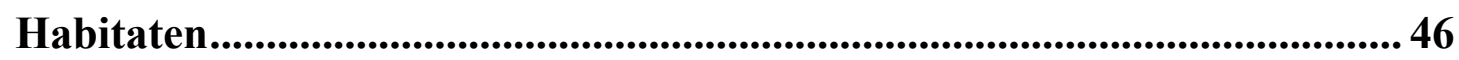

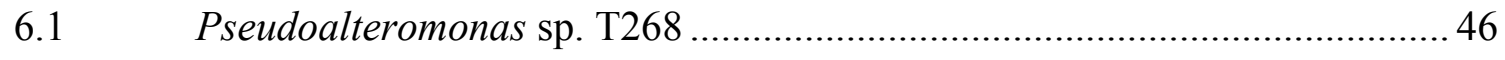

6.1.1 Bestimmung der Taxonomie ...................................................................... 46

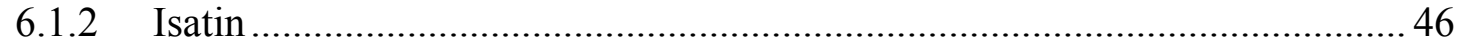

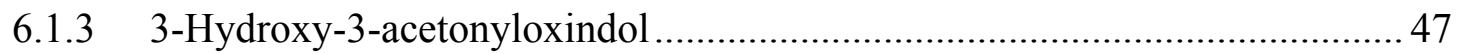

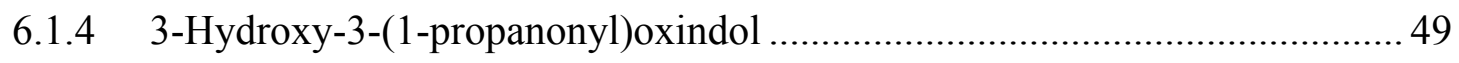

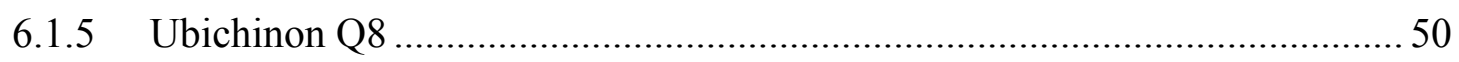




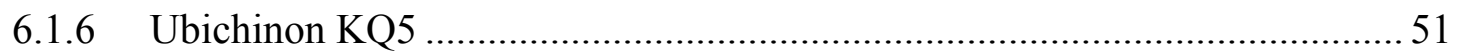

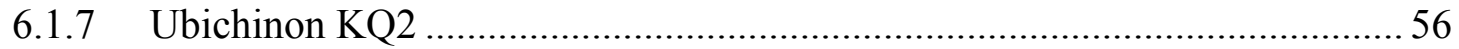

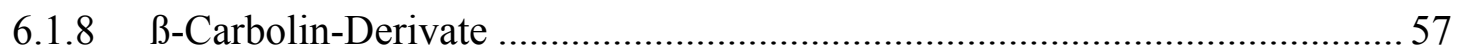

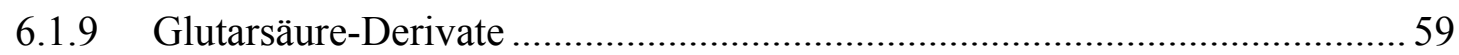

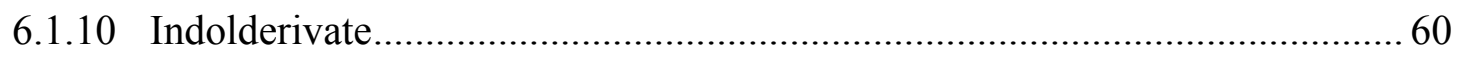

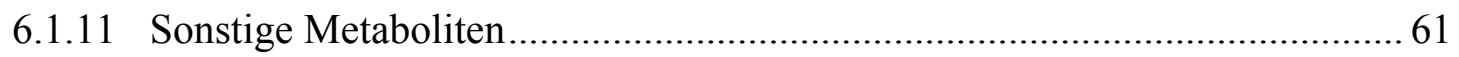

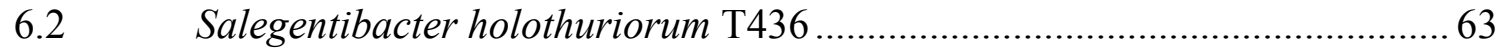

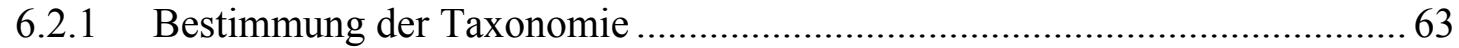

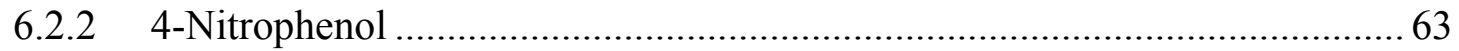

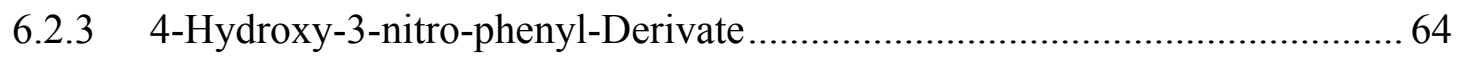

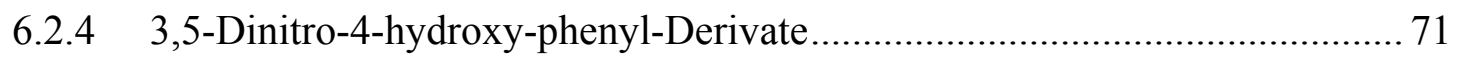

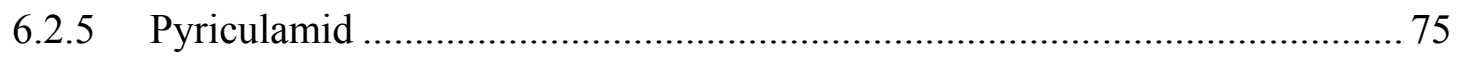

6.2.6 7-Hydroxy-3-(4-hydroxy-3-nitrophenyl)-4H-1-benzopyran-4-on (3'-Nitro-

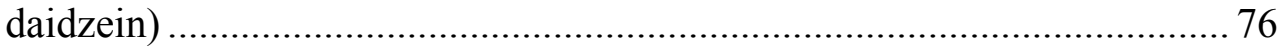

6.2.7 5,7-Dihydroxy-3-(3,5-dinitro-4-hydroxy-phenyl)-4H-1-benzopyran-4-on

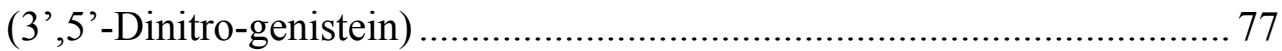

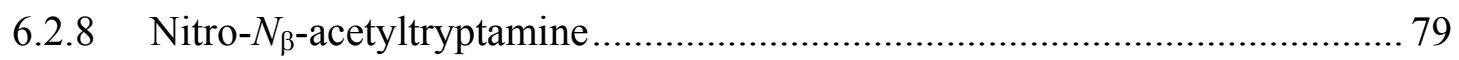

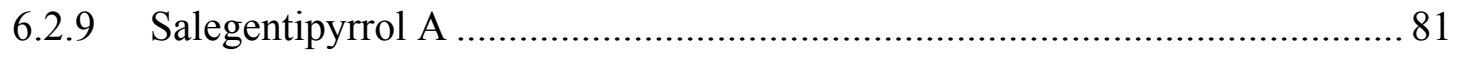

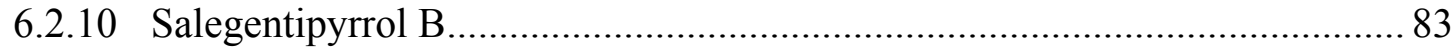

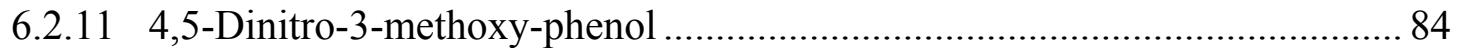

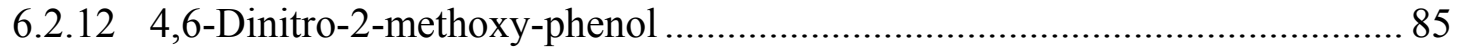

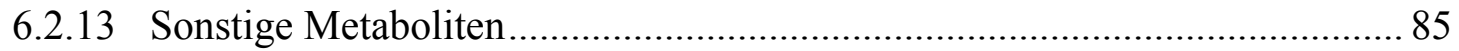

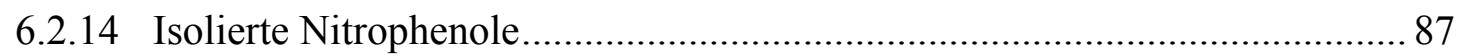

6.2.15 ESI-Fragmentierung der aromatischen Nitroverbindungen ......................... 91

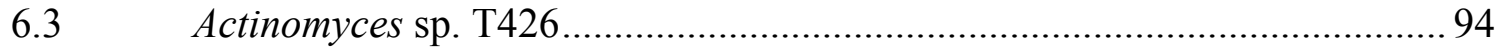

6.3.1 Tryptophol und $p$-Hydroxybenzoesäuremethylester .................................... 94

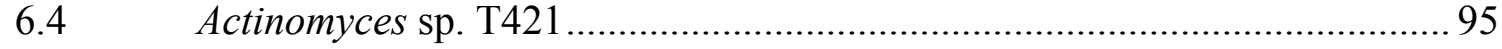

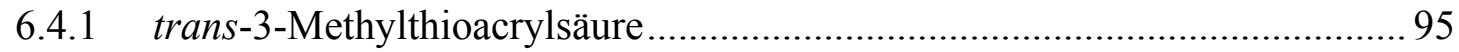

6.4.2 Benzoesäure und Phenylessigsäure ............................................................. 96

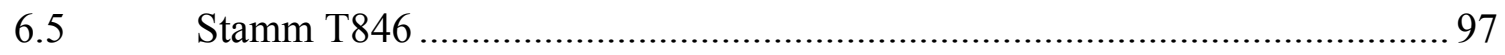

6.5.1 3-Indolylcarbonsäure und 3-Indolylcarbaldehyd ......................................... 97

6.5.2 3-(Methylthio)propansäure ……............................................................... 97

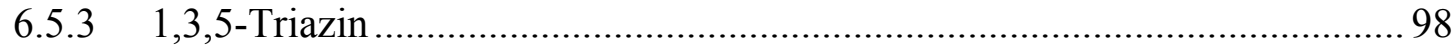

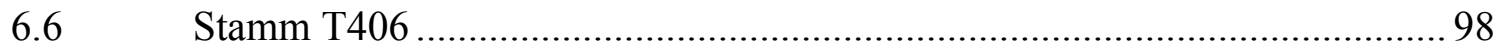


6.6.1 2-(Indol-3-ylmethyl)-indol-3-ylethanol …................................................. 99

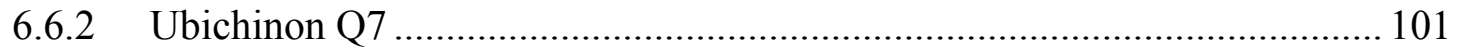

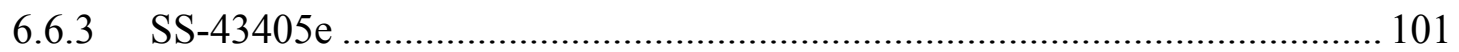

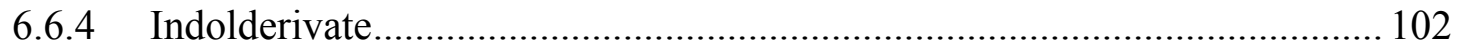

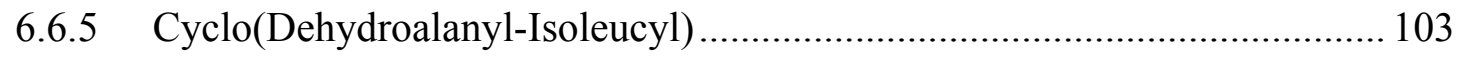

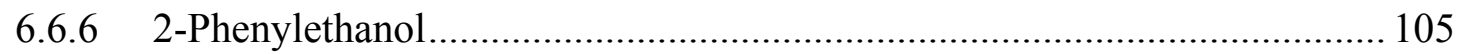

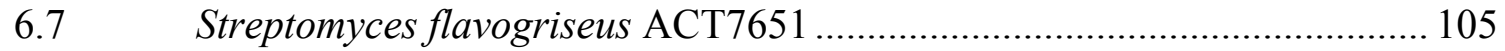

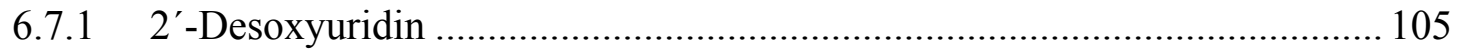

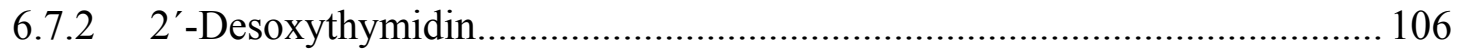

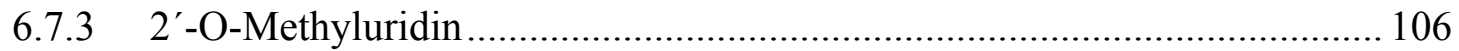

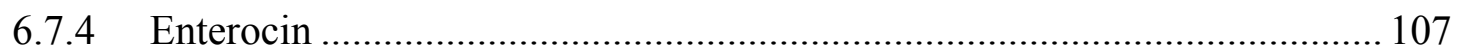

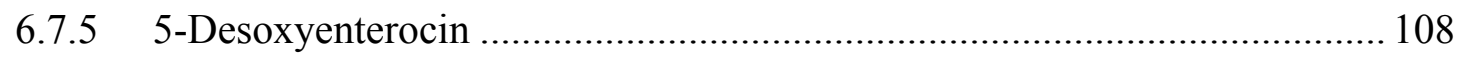

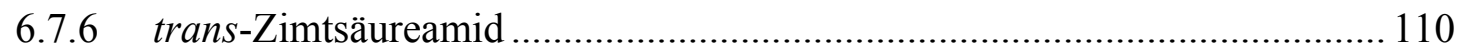

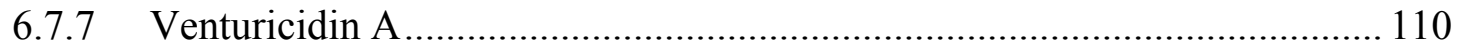

7 Untersuchungen ausgewählter Stämme aus anderen Habitaten............... 112

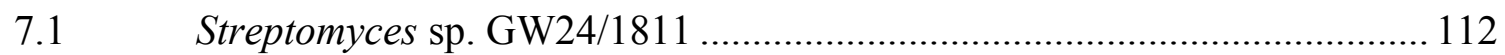

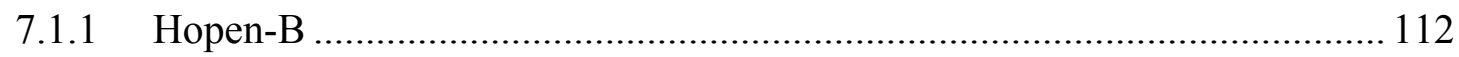

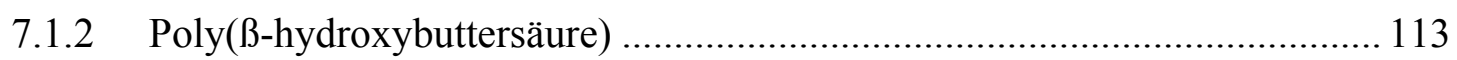

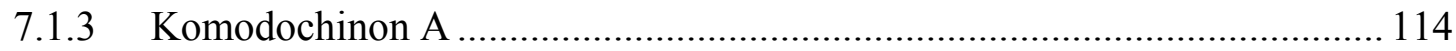

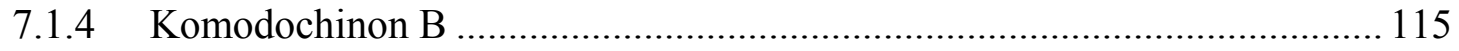

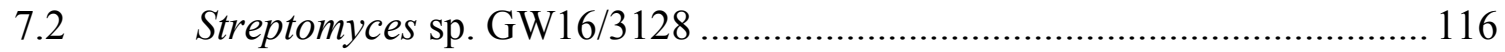

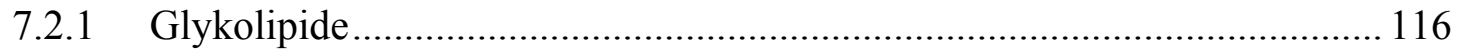

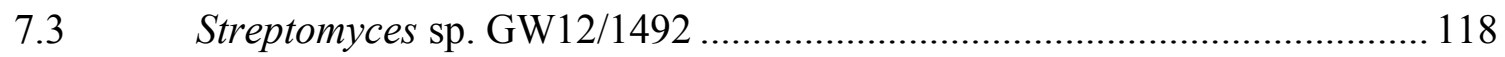

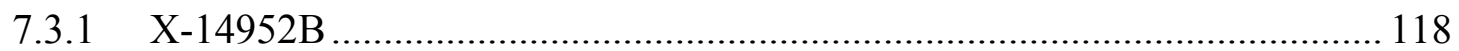

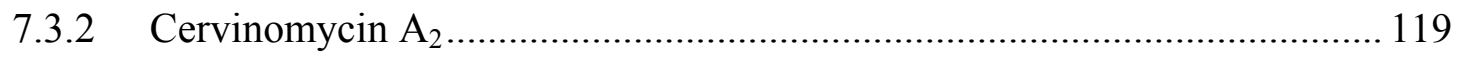

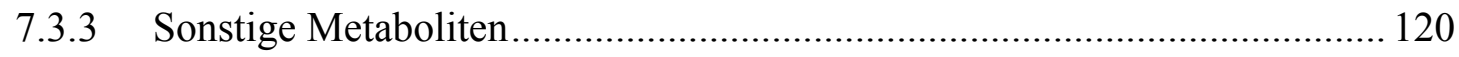

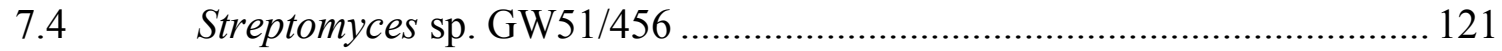

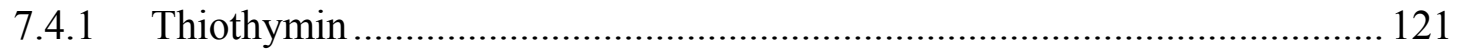

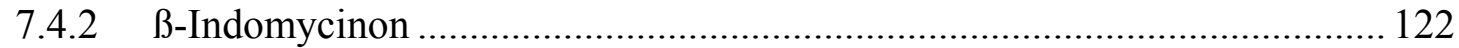

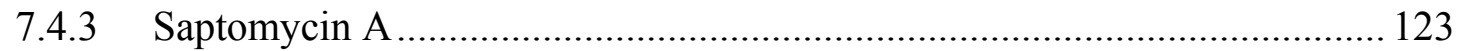

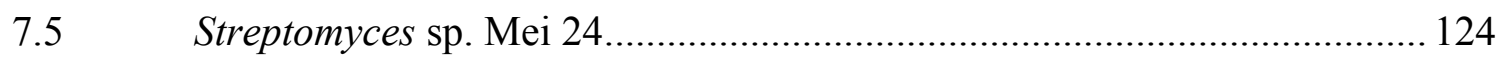

7.5.1 4-Methylamino-7-methyl-isoquinolin-3,6-dion ........................................ 124

7.5.2 4-Methylamino-7,8-dimethyl-isoquinolin-3,6-dion .................................. 124 
$8 \quad$ Zusammenfassung.................................................................................................... 126

9 Materialien und Methoden............................................................................. 134

10 Experimenteller Teil ........................................................................................... 142

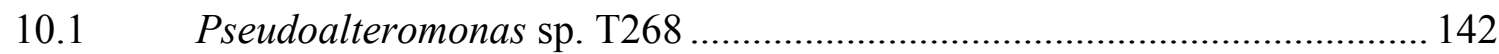

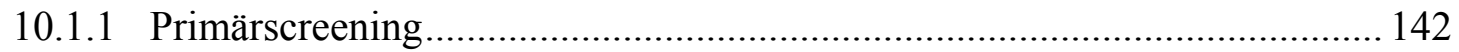

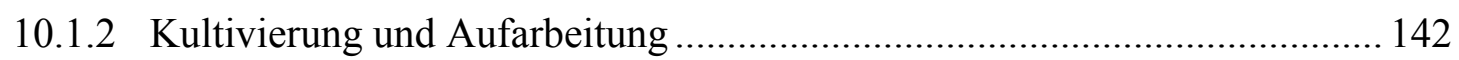

10.1.3 Isolierung und Charakterisierung der Metaboliten...................................... 143

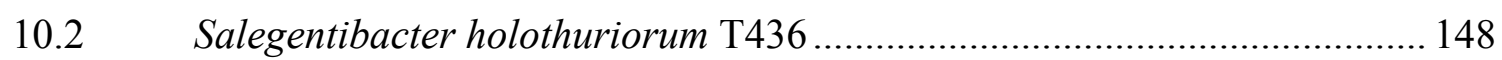

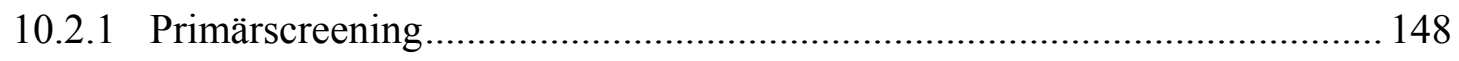

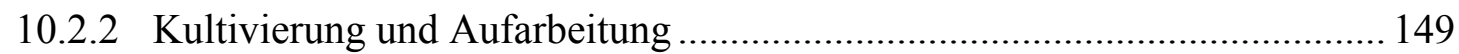

10.2.3 Isolierung und Charakterisierung der Metaboliten..................................... 149

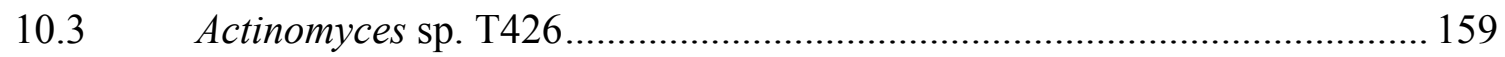

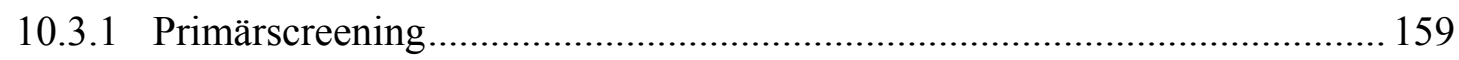

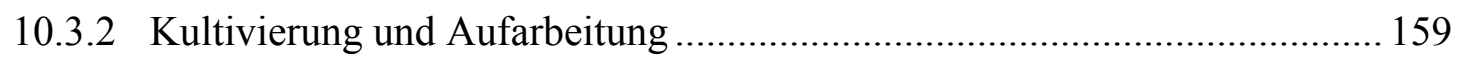

10.3.3 Isolierung und Charakterisierung der Metaboliten..................................... 160

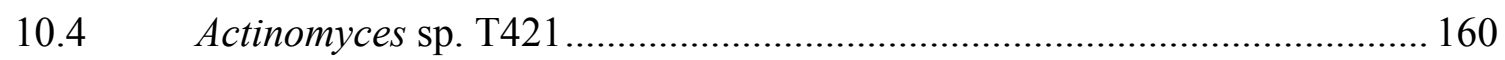

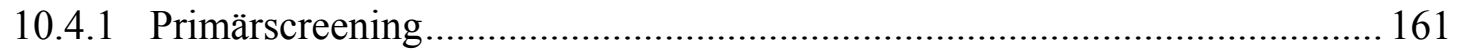

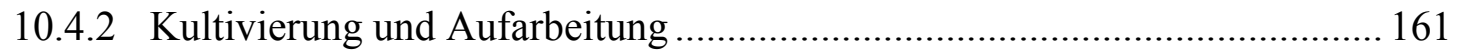

10.4.3 Isolierung und Charakterisierung der Metaboliten...................................... 161

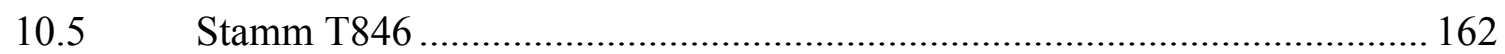

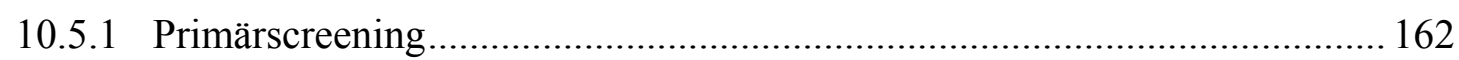

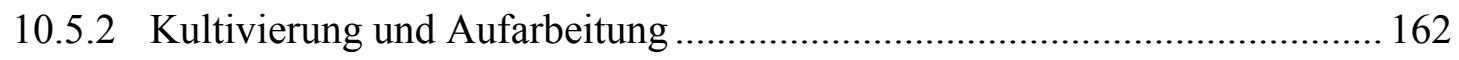

10.5.3 Isolierung und Charakterisierung der Metaboliten.................................... 162

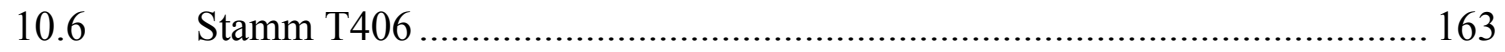

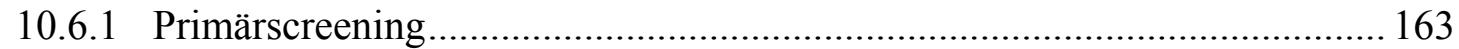

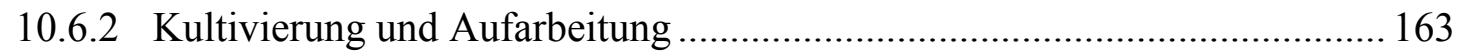

10.6.3 Isolierung und Charakterisierung der Metaboliten..................................... 163

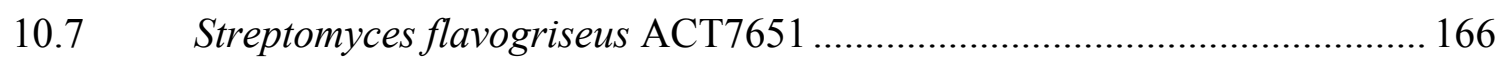

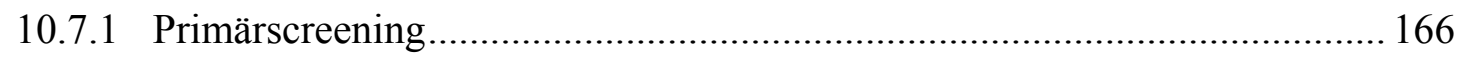

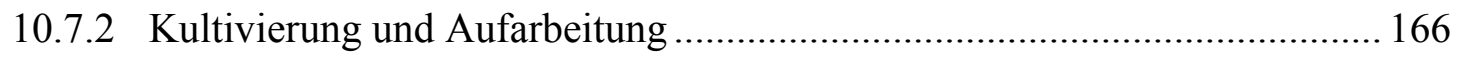

10.7.3 Isolierung und Charakterisierung der Metaboliten..................................... 167 


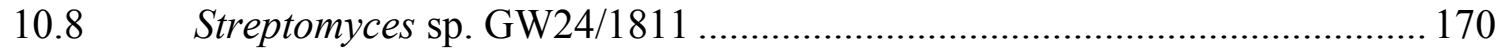

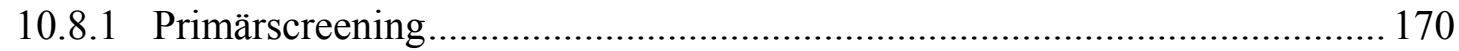

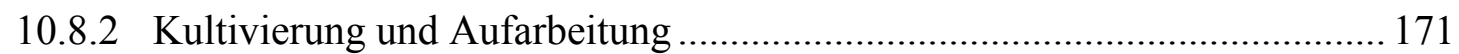

10.8.3 Isolierung und Charakterisierung der Metaboliten........................................ 171

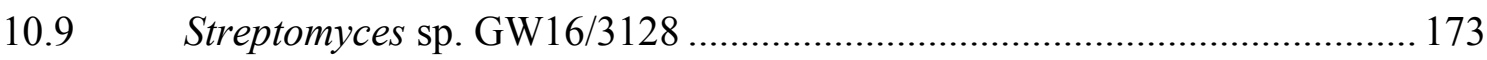

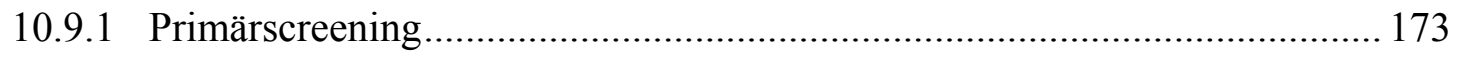

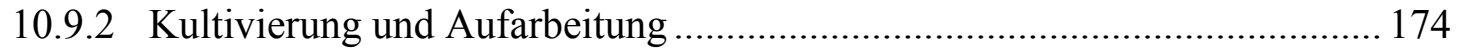

10.9.3 Isolierung und Charakterisierung der Metaboliten........................................ 174

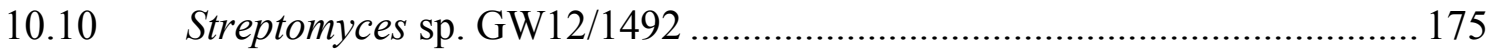

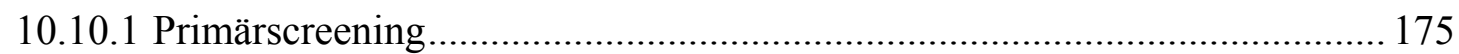

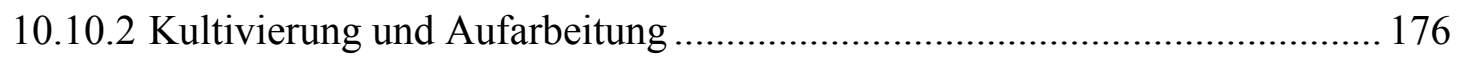

10.10.3 Isolierung und Charakterisierung der Metaboliten....................................... 176

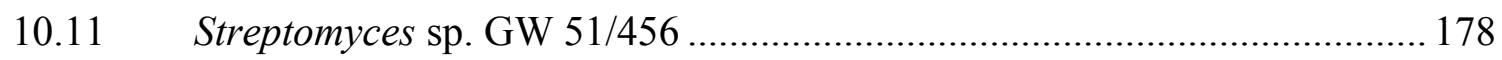

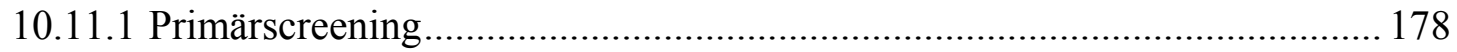

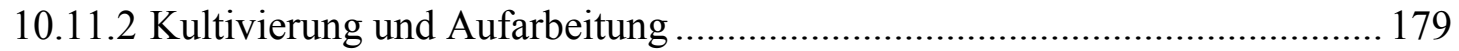

10.11.3 Isolierung und Charakterisierung der Metaboliten........................................ 179

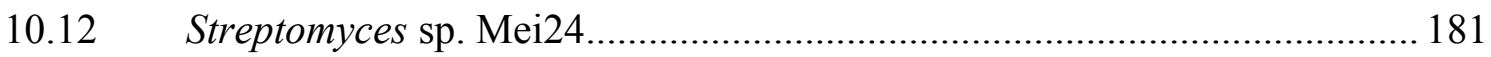

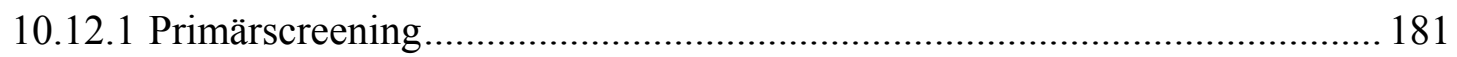

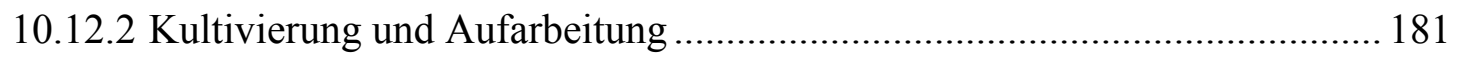

10.12.3 Isolierung und Charakterisierung der Metaboliten....................................... 182

11 Literaturverzeichnis........................................................................................... 183 


\section{$1 \quad$ Einleitung}

Die Untersuchung von Naturstoffen findet an der Schnittstelle zwischen Biologie und Chemie statt. Durch die Methoden der modernen Forschung ist die Aufklärung der molekularen Strukturen, welche den biologisch relevanten Stoffen zugrunde liegen, in hohem Maße vereinfacht worden. Dies trug maßgeblich dazu bei, dass man heute ein recht detailliertes Verständnis der von der Natur zum Aufbau chemischer Verbindungen mit völlig unterschiedlichen Aufgabenbereichen genutzten Prinzipien hat. Der Zusammenhang zwischen der chemischen Struktur einerseits und den resultierenden biologischen Eigenschaften andererseits ist jedoch sehr komplex und bis heute Gegenstand intensiver Forschung.

In vielen Fällen bringen insbesondere die im Sekundärmetabolismus von Pflanzen und Tieren gebildeten Naturstoffe dem produzierenden Organismus einen entscheidenden Vorteil beim täglichen Kampf ums Überleben, wenngleich der ursächliche Zusammenhang im Detail oft nicht geklärt ist. Bis heute ist dabei die überwiegende Zahl an Naturstoffen aus Pflanzenextrakten isoliert worden. Betrachtet man solche Sekundärmetaboliten im Hinblick auf ihre mögliche Eignung als Arzneimittel, fällt auf, dass Pflanzen nur bedingt als Produzenten hochwirksamer Antibiotika auftreten. Letzteres ist vielmehr eine Domäne der Mikroorganismen.

Darüber hinaus erweisen sich Bakterien und Pilze, die als einzige Spezies alle Lebensräume erobert haben, unabhängig von ihrer phylogenetischen Einordnung als Produzenten einer weiten Bandbreite interessanter Substanzen. In den letzten Jahren galt die besondere Aufmerksamkeit der Naturstoffchemie vor allem der Untersuchung terrestrischer Actinomyceten und Pilze. Die Suche nach neuen, biologisch aktiven Naturstoffen hat das Interesse in jüngster Zeit jedoch verstärkt auf solche Organismen gerichtet, welche sich als Bewohner bisher wenig untersuchter Habitate auszeichnen. Ihre besondere Anpassungsfähigkeit, mitunter auch an extremste Bedingungen, lässt vermuten, dass sie zur Produktion von Substanzen mit spezieller Wirksamkeit befähigt sind.

Naturstoffe verfügen über eine hohe strukturelle Diversität. Fast alle bekannten Strukturelemente sind vertreten, wobei die zugrunde liegenden Molekülgrößen über einen großen Bereich variieren. Um diese große Substanzklasse einer effizienten Untersuchung zugänglich zu machen und die biologische Aktivität neu gefundener Verbindungen rasch ermitteln zu können, bedient man sich heute der Methode des High-Throughput-Screening (HTS). 


\subsection{Naturstoffe aus marinen Organismen}

Seit Beginn der siebziger Jahre des vergangenen Jahrhunderts wurden mehr als 10000 strukturell unterschiedliche Naturstoffe aus marinen Organismen isoliert, welche durch eine Reihe biologischer Aktivitäten auffielen. ${ }^{1}$ Die größte Gruppe unter allen marinen Lebewesen bilden die Mikroorganismen, wobei sich die Zahl der vorkommenden Arten nur schwer abschätzen lässt. Es wird zudem vermutet, dass viele Wirkstoffe aus höheren marinen Organismen zu einem Großteil von mit ihnen assoziiert auftretenden Mikroorganismen gebildet werden. Bei der Betrachtung der isolierten Substanzen fällt auf, dass diese bereits teilweise aus terrestrischen Quellen bekannt sind.

Bislang erweist sich die medizinische Anwendung mariner Naturstoffe aus verschiedenen Gründen als problematisch. So können in diesem Kontext vielversprechende Substanzen oft nur in sehr geringen Mengen isoliert werden. Um die begrenzten natürlichen Ressourcen zu schonen, ist man auf eine Kultivierung der betreffenden Organismen im Labor angewiesen, welche sich insbesondere bei Invertebraten und/oder deren Symbionten als schwierig erweist. Hauptsächlich dann, wenn es um die Fermentation von Mikroorganismen geht, erweist sich die Etablierung geeigneter Systeme, welche die komplexen, marinen Umweltbedingungen im Labormaßstab nachbilden, als limitierender Faktor. Trotz dieser Hindernisse sind einige marine Naturstoffe bereits Gegenstand klinischer bzw. präklinischer Studien.

Tabelle 1 Beispiele von marinen Naturstoffen, welche sich in der klinischen oder präklinischen Phase befinden. ${ }^{2,3}$

\begin{tabular}{|c|c|c|c|}
\hline Substanz & Organismen & Krankheit & $\begin{array}{c}\text { Klinische } \\
\text { Phase } \\
\end{array}$ \\
\hline Ziconotide (Prialt ${ }^{\mathrm{TM}}$ ) & Conus magnus (Molusk) & Neuropathien & III \\
\hline $\begin{array}{l}\text { Ecteinascidin } 743 \\
\left(\text { Yondelis }^{\mathrm{TM}} \text { ) }\right.\end{array}$ & Ecteinascidia turbinata (Tunikate) & Krebs & II/III \\
\hline Bryostatin 1 & Bugula neritina (Bryozoon) & Krebs & II \\
\hline Dolastatin 10 & $\begin{array}{l}\text { Dolabella auricularia (Molusk, } \\
\text { später in Cyanobakterien gefunden) }\end{array}$ & Krebs & II \\
\hline IPL-576092 & Petrosia contignata (Schwamm) & Psoriasis & II \\
\hline IPL-512602 & Petrosia contignata (Schwamm) & $\begin{array}{l}\text { Asthma/ } \\
\text { Entzündung }\end{array}$ & II \\
\hline Thiocoraline & $\begin{array}{l}\text { Micromonospora marina } \\
\text { (Bakterium) }\end{array}$ & Krebs & präklinisch \\
\hline
\end{tabular}


Bei dem ersten marinen Naturstoff, der sich als Krebsmittel in klinischen Untersuchungen befand, handelt es sich um Didemnin B (1). Dieser Wirkstoff konnte bereits im Jahre 1981 aus Tunikaten isoliert werden. ${ }^{4}$

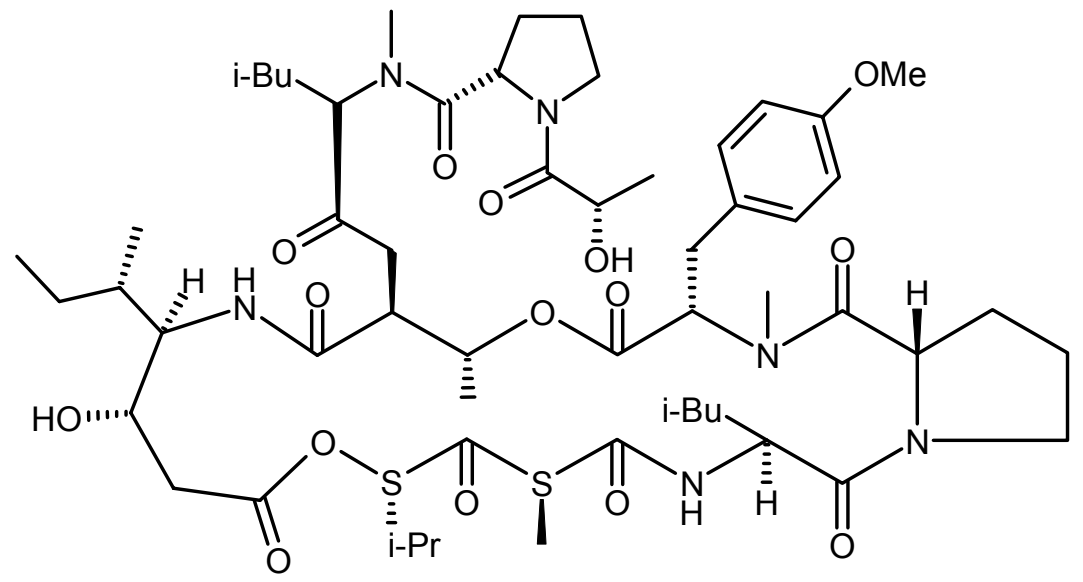

1

Das Antikrebsmittel Bryostatin 1 (2), welches zum ersten Mal in den siebziger Jahren als Metabolit des Bryozoon Bugula neritina gefunden wurde, konnte erst im Jahre 1982 per Röntgenkristallographie in seiner Struktur vollständig aufgeklärt werden. ${ }^{5}$ Neuere Befunde deuten auf einen mikrobiellen Ursprung als Quelle von 2 hin, was die Möglichkeit zur Kultivierung des produzierenden Symbionten eröffnen könnte. ${ }^{6}$<smiles>CCC/C=C/C=C/C(=O)O[C@H]1/C(=C/C(=O)OC)C[C@H](C[C@@H](O)[C@H](C)O)OC1(O)C(C)(C)/C=C/C1C/C(=C/C(=O)OC)CC(C[C@]2(O)OC(C[C@H](O)CC(=O)OC)C[C@H](OC(C)=O)C2(C)C)O1</smiles>

2

Ein anderes Beispiel für einen marinen Naturstoff mit interessanter biologischer Aktivität ist das aus dem Dinoflagellaten Gambierdiscus toxicus isolierte Maitotoxin (3). ${ }^{7,8}$ Diese zur Klasse der Polyether gehörende Verbindung zählt zu den potentesten bislang gefun- 
denen Giften und stellt das nicht-peptidische Toxin mit dem größten bekannten Molekulargewicht dar. Zur Strukturaufklärung wurde das Molekül chemisch gespalten und die erhaltenen Bruchstücke wurden anschließend per HPLC getrennt und mittels NMR-Spektroskopie analysiert.
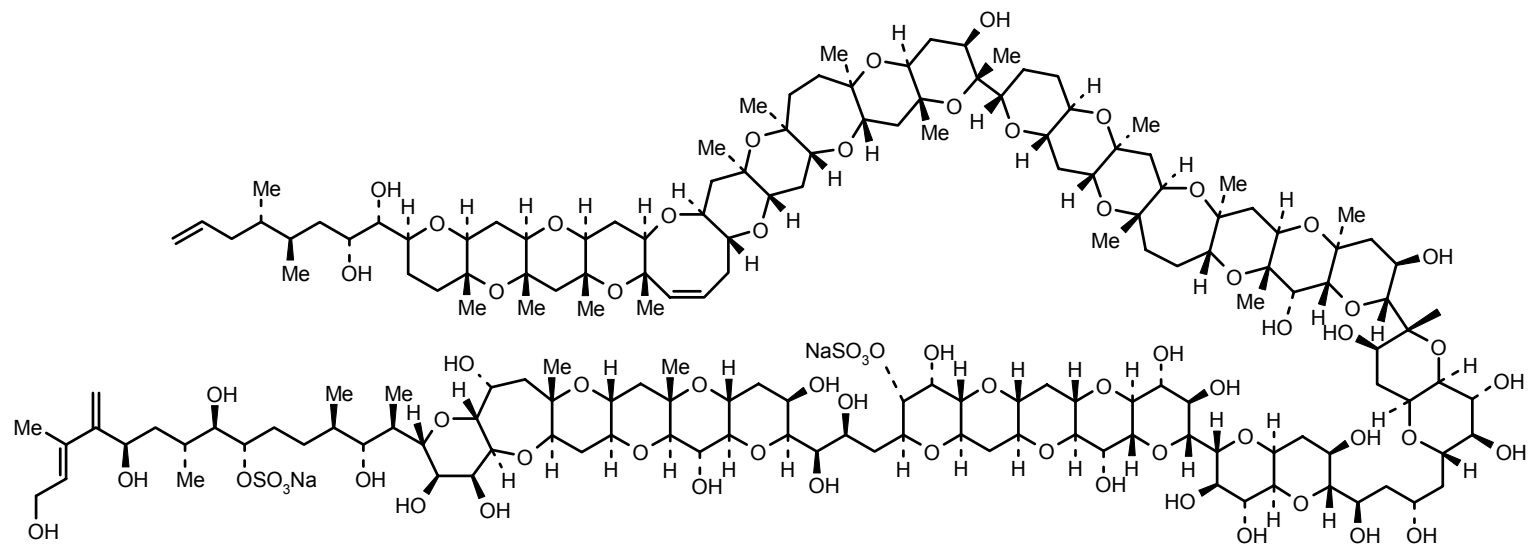

\subsection{Arktische und antarktische Meereisbakterien}

Ein außergewöhnliches marines Habitat bildet das arktische und antarktische Eis. Hier findet man neben extremophilen Mikroorganismen wie Bakterien und Algen auch höher entwickelte Lebewesen wie Krebse und Würmer. Offenbar bietet Meereis für das Wachstum von Mikroorganismen günstige Voraussetzungen, auf gleiche Volumina bezogen ist es sogar dichter besiedelt als Nordseewasser während der Algenblüte. ${ }^{9}$

Meereis wird aus zunächst entstehenden Süßwassereiskristallen gebildet, welche unter dem Einfluss von Wind und Wellen zu kompakterem Eis zusammengepresst werden. Bei diesem Prozess wird kontinuierlich Salzwasser eingeschlossen, aus dem wiederum Süßwasser auskristallisieren kann. Ein Teil der dabei gebildeten konzentrierten Sole sinkt aufgrund ihrer höheren Dichte während der Eisbildung ins Meerwasser, ein anderer verbleibt in abgeschlossenen Eiszwischenräumen im Eis. In den sogenannten Salzlaugenkanälen reichert sich also eine hochgesättigte Salzlösung an, deren Konzentration nach Temperatur und Eishöhe abhängt. In den sich hier bietenden ungewöhnlichen Lebensräumen finden besonders angepasste Kieselalgen, Dinoflagellaten, Mikroalgen, Pilzen und Bakterien eine ökologische Nische. 
Kälteadaptierte Mikroorganismen, welche nur in einem Temperaturbereich von ca. -2 bis $20{ }^{\circ} \mathrm{C}$ existieren können, bezeichnet man als psychrophil. Das Wachstumsoptimum findet man hier zwischen 4 und $10{ }^{\circ} \mathrm{C}$. Psychrotrophe Arten hingegen können auch Temperaturen bis $40{ }^{\circ} \mathrm{C}$ tolerieren. Mikroorganismen, die an normale (mesophile) Bedingungen angepasst sind und trotzdem kurzzeitige Kälteperioden ohne Schaden überdauern, werden als psychrotolerant bezeichnet.

Über die niedermolekularen Stoffwechselprodukte, die von kälteadaptierten Organismen produziert werden, ist derzeit noch recht wenig bekannt. Ein Beispiel an dieser Stelle ist die Verbindung $N_{B}$-Acetylkynuramin (4), die in unserer Arbeitsgruppe aus antarktischem Meereisbakterium ANT V/2 253 isoliert und identifiziert wurde. ${ }^{10}$<smiles>CC(=O)NCCC(=O)c1ccccc1N</smiles>

4

Andere Beispiele sind die Verbindungen Haliclamin C (5) und D (6) genannt, die zur Substanzklasse der 3-Alkyltetrahydropyridin-Alkaloide gehören und als Metabolite des arktischen Schwamms Haliclona viscosa isoliert wurden. ${ }^{11}$

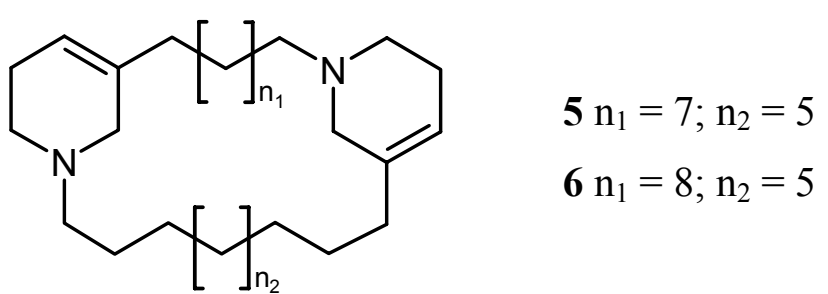

Weitere Vertreter von Stoffwechselprodukten kälteadaptierter Organismen sind die aus Pseudomonas fluorescens 51W isolierten Verbindungen Pyoverdin 51W (7), sucPyoverdin 51W und suca-Pyoverdin 51W. ${ }^{12}$ Diese Substanzen bilden Chelatkomplexe mit Eisen(III)-Ionen, was die Aufnahme dieses essentiellen Elements durch die Zelle erleichtert. Die Produktion solcher als Siderophore bezeichneten Chelatbildner wird unter Eisenmangelbedingungen induziert; bei den Eisenkomplexen handelt es sich meist um rotbraun gefärbte Verbindungen. Die auffällige gelb-grüne Farbe der fluoreszierenden Pseudomonaden ist jedoch durch Pyoverdine bedingt. ${ }^{13}$ 
<smiles>CC(NC(=O)C(CO)NC(=O)C(CCC(N)=O)NC(=O)C(NC(=O)CNC(=O)CNC(=O)C(CCCCN)NC(=O)C(C)NC(=O)C1CCNc2c(NC(=O)CCC(=O)C(=O)O)cc3cc(O)c(O)cc3[n+]21)C(O)C(=O)O)C(=O)NCC(=O)NC(C(=O)NC1CCCN(O)C1=O)C(C)O</smiles>

\subsection{Symbiotische Mikroorganismen}

Mikroorganismen treten oft im Verbund mit höher entwickelten Lebewesen auf. Der Begriff „Symbiose“ wird im klassischen Sinn verwendet, um Beziehungen zwischen zwei Organismen zu beschreiben, die unterschiedlichen Spezies angehören und physikalisch nahe assoziiert auftreten. Eine für beide Seiten nützliche Symbiose wird dabei als Mutualismus bezeichnet. Profitiert nur ein Organismus von der Lebensgemeinschaft und geht diese zu Lasten des anderen, so spricht man von parasitären Verhältnissen. Im Fall von pathogenen Keimen führen diese zusätzlich zu einer Erkrankung des befallenen Wirts. Die Forschung auf dem Gebiet der symbiotisch lebenden Bakterien und Pilze ist durch den Umstand, dass es sich hierbei vielfach um nur schwer oder nicht kultivierbare Mikroorganismen handelt, stark beeinträchtigt.

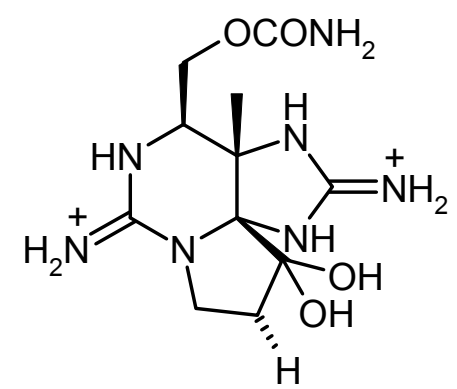

8

Ein Beispiel für einen Naturstoff, welcher von symbiotisch mit Dinoflagellaten assoziierten Mikroorganismen gebildet wird, stellt Saxitoxin (8) dar. Während der Dinoflagellaten-Blüte, die auch als ,rote Schleife“ bekannt ist, kommt es zur Freisetzung verschiedener Gifte in die marine Nahrungskette, wobei 8 eine wesentliche Komponente 
darstellt. Der Verzehr von Fischen und Schalentieren, in denen diese Toxine angereichert sind, kann zu einer Reihe von Vergiftungen beim Menschen wie beispielsweise dem PSP (paralytic shellfish poisoning) führen. ${ }^{14}$ KODAMA und Mitarbeiter konnten bereits 1988 ein intrazelluläres Bakterium aus dem Dinoflagellaten Alexandrium tamarense isolieren, welches Saxitoxin $(\mathbf{8})$ produziert. ${ }^{15}$ Seitdem wurden zahlreiche weitere Toxin produzierende Bakterien aus Dinoflagellaten isoliert. ${ }^{16}$<smiles>O=C1Nc2ccccc2C1=O</smiles>

9<smiles>OCCc1ccc(O)cc1</smiles>

10

Extern ausgebrütete Embryonen der Shrimpsart Palaemon macrodactylus sind resistent gegen Infektionen, die von dem Pilz Lagenidium callinectes hervorgerufen werden. FENICAL und Mitarbeiter fanden ${ }^{17}$, dass eine bestimmte Alteromonaden-Art, die auf der Oberfläche der gesunden Embryonen lokalisiert ist, Isatin (9) produziert. Diese Verbindung hemmt das Wachstum von L. callinectes. Ein ähnlicher Abwehrmechanismus, welcher auf von Gram-negativen Bakterien produziertem Tyrosol (10) beruht, wurde auch bei Hummer-Embryonen gefunden. ${ }^{18}$

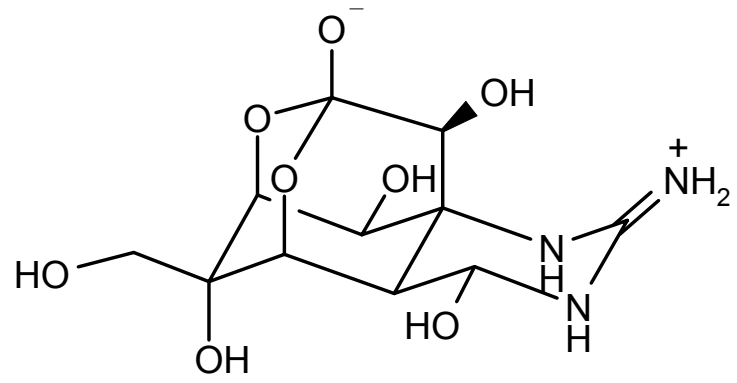

11

Fugu-Fisch ist eine Delikatesse in der japanischen Küche. Da sowohl Eierstöcke als auch Leber dieser Fischart hoch toxisch sind, darf sie nur von speziell ausgebildeten Köchen zubereitet und serviert werden. Das enthaltene Neurotoxin, welches zur Blockierung der spannungsabhängigen Natrium-Kanäle führt, ${ }^{19}$ wurde als Tetrodotoxin (11) identifiziert. ${ }^{20}$ Diese Verbindung konnte ebenfalls als Metabolit von Bakterien gefunden werden, welche man aus dem Fisch isolierte. ${ }^{21,22}$ 
Pathogene Keime bedienen sich vielfach der Hilfe von Siderophoren, um durch Chelatbildung die Aufnahme essentieller Eisenionen aus dem Wirt zu ermöglichen. Dieses im Wirt in vergleichsweise großer Menge vorhandene Element ist stark an intra- und extrazelluläre Komponenten gebunden. ${ }^{23}$ Sinkt die Menge an freien Eisenionen unter einen bestimmten Wert, kann das pathogene Bakterium seinen Bedarf nicht mehr decken und stirbt ab. ${ }^{24}$ Aus diesem Grund können höhere Organismen die Konzentration freier Eisenionen im Zuge einer Abwehrreaktion bei einer Infektion bis auf Werte reduzieren, die noch keinen Einfluss auf lebenswichtige Funktionen beim Wirt haben, eine Ausbreitung der Bakterien aber erschweren. Ein Vertreter eines solchen eisenabhängigen pathogenen Keims ist das Pyochelin (12) produzierende Bakterium Pseudomonas aeruginosa, welches eine Infektion der menschlichen Lunge verursachen kann. ${ }^{25}$

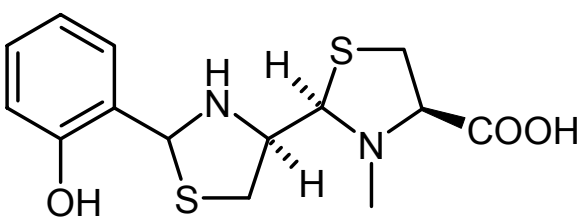

12

Einige Pflanzen, insbesondere Apfelbäume, produzieren bei einer Infektion der Blätter (Apfelschorf) oder der Haarwurzeln die sog. Opine, weil die für deren Biosynthese verantwortlichen Gene über ein bakterielles Plasmid in das pflanzliche Genom eingeschleust wurden. ${ }^{26}$ Die Opine wiederum werden von Parasiten wie Agrobacterium tumefaciens und A. rhizogenes als Wachstumsfaktoren benötigt. Eine derartige PflanzenBakterien-Beziehung ist ein Beispiel für eine natürliche genetische Manipulation. 


\section{$2 \quad$ Aufgabenstellung}

Ökologische oder symbiotische Wechselwirkungen sind also überwiegend chemischer Natur, wobei sicherlich auch die Habitate der jeweiligen Organismen eine gewichtige Rolle spielen. Die Aufklärung der zugrunde liegenden Mechanismen wird durch die Komplexität ökologischer Systeme allerdings erheblich erschwert, weshalb am Anfang Untersuchungen an Modellsystemen oder zumindest einfachen Lebensgemeinschaften sinnvoll sind. Die Aufklärung einer chemischen Kommunikation erfordert immer erst die Bilanzierung des chemischen Vokabulars, welches unter anderem aus mikrobiellen Sekundärmetaboliten aufgebaut ist. Die vorliegende Arbeit beschäftigt sich mit der Untersuchung polarer Mikroorganismen, welche aufgrund ihres Habitates von besonderem Interesse sind. So handelt es sich bei Arktis und Antarktis um einzigartige, nahezu abgeschlossene Ökosysteme, deren Lebensgemeinschaften im Hinblick auf ökologische und symbiotische Zusammenhänge bisher nur wenig untersucht wurden. Zudem bestehen in unserer Arbeitsgruppe bereits erste Erfahrungen im Umgang mit polaren Mikroorganismen, ${ }^{10}$ welche eine gute Basis für weitere Arbeiten auf diesem Gebiet darstellen.

Die für die Untersuchungen benötigten Bakterienstämme sind über bestehende Zusammenarbeiten mit dem Institut für Biotechnologie und Wirkstoffforschung (Kaiserslautern) und dem Alfred-Wegener-Institut (Bremerhaven) zugänglich. Rohextrakte müssten nach einem biologischen und chemischen Screening chromatographisch aufgetrennt und die Strukturen der so erhaltenen Reinsubstanzen mit geeigneten Methoden aufgeklärt werden.

Voraussetzung für die geplanten Arbeiten ist eine leistungsfähige Analytik, für die sich aus verschiedenen Gründen besonders die ESI-Massenspektrometrie in Kombination mit weiteren analytischen Methoden, wie UV-Spektroskopie und HPLC, anbietet.

Mithilfe dieser Techniken ist zunächst eine HPLC-UV-ESI-MS/MS-Datenbank von mikrobiellen Sekundärmetaboliten aufzubauen, die in unserer Arbeitsgruppe isoliert wurden. Die erstellte Datenbank würde anschließend nicht nur zur Dereplikation bekannter Verbindungen im Screening von Meereisbakterien nutzbar sein, sondern sich allgemein auch zur Dereplikation von Metaboliten aus anderen Bakterienstämmen eignen.

Es liegt nahe, die erhaltenen Daten zu einer genaueren Untersuchung von ESI-Fragmentierungswegen unterschiedlicher Substanzklassen zu nutzen, um auch die Strukturzuordnung bislang nicht bekannter bzw. nicht in der Datenbank vorhandener Substanzen zu ermöglichen. 
Einen weiteren Aspekt der vorliegenden Arbeit bildet die Strukturaufklärung von Sekundärmetaboliten aus terrestrischen sowie marinen Actinomyceten-Stämmen, deren Rohextrakte sich durch biologische Aktivität bzw. interessante Zonen im chemischen Screening auffällig zeigen. Die vom Institut für Bodenkunde (Lohra-Kirchvers), dem Alfred-Wegener-Institut (Bremerhaven) und der Fachhochschule Ostfriesland (Emden) bereitgestellten Stämme sollten in größerem Maßstab kultiviert und die gebildeten Sekundärmetaboliten mithilfe chromatographischer Methoden isoliert und in ihrer Struktur aufgeklärt werden. 


\section{$3 \quad$ Screening der Bakterienstämme}

Die von der Natur zur Synthese bakterieller Sekundärstoffe verwendeten Mechanismen ermöglichen eine nahezu unbegrenzte Strukturvielfalt, welche ein enormes Potenzial an unterschiedlichsten biologischen Aktivitäten birgt. Um das Metabolitenspektrum eines Stammes beurteilen und geeignete Produzenten auswählen zu können, führt man zunächst ein Screening durch. Dies kann wirkungsorientiert als biologisches Screening mit Hilfe lebender Organismen oder entsprechend angepasster Modelle erfolgen oder im Fall des chemischen Screening auf bestimmten chemischen bzw. physikalischen Stoffeigenschaften basieren. Zur experimentellen Durchführung bedient man sich der Rohextrakte der zu untersuchenden Stämme. Diese wurden in der vorliegenden Arbeit durch Kultivierung in Schüttelkolben und anschließende standardisierte Aufarbeitung der Kulturbrühen erhalten.

\subsection{Biologisches Screening}

Die Prüfung der biologischen Aktivität eines Rohextraktes bzw. bereits vorgetrennter Fraktionen gegenüber bestimmten Testorganismen (z. B. Bakterien, Pilze, Algen und Zelllinien) kann in Form eines Plattendiffusionstests erfolgen. In Abhängigkeit von der Größe des sich ergebenden Hemmhofes kann hier eine Beurteilung hinsichtlich der Aktivität einer Substanz bzw. eines Extrakts vorgenommen werden. Zur Bewertung der Cytotoxizität gegenüber höheren Organismen dienen Tests z. B. mit Salinenkrebsen (Artemia salina) und Nematoden (Caenorhabditis elegans), wobei die cytotoxische Aktivität hier anhand der Mortalitätsrate der Larven bestimmt wird. Als Nachteil dieses Screeningsansatzes erweist sich dessen Limitierung auf die verwendeten Testsysteme. Wird ein Extrakt als nicht aktiv eingestuft, erfolgt dessen Aussortierung. Dies birgt allerdings die Gefahr, dass Substanzen mit einem im Testsystem nicht erfassten Wirkspektrum wegen der vermeintlich fehlenden biologischen Aktivität nicht weiter bearbeitet werden. Als weiterhin problematisch erweisen sich falsch positive Ergebnissen, welche beispielsweise auf synergistischen Effekten im untersuchten Substanzgemisch beruhen, sowie (hoch)aktive Substanzen, welche aufgrund ihrer gering Konzentration nicht in ausreichenden Mengen isoliert werden können.

In neuerer Zeit finden verstärkt Target-orientierte Testsysteme Verwendung, welche allerdings ein detailliertes Verständnis von Krankheitsursachen und -symptomen auf molekularer Ebene voraussetzen. Man bedient sich hier Rezeptor- oder Enzym-basierter 
Assays, welche im High-Throughput-Verfahren betrieben werden und ein zeit- und kosteneffizientes industrielles Screening großer Substanzbibliotheken ermöglichen. Durch sorgfältige Auswahl der verwendeten Target-Systeme kann hiermit gezielt nach solchen Stoffen gesucht werden, welche als Therapeutika mit ursächlichem Wirkprinzip zur effizienten Behandlung bestimmter Erkrankungen eingesetzt werden können.

\subsection{Chemisches Screening}

Im klassischen chemischen Screening werden Extrakte dünnschichtchromatographisch untersucht, wobei substanzspezifische Parameter wie Eigenfarbe, UV-Löschung, $\mathrm{R}_{\mathrm{f}}$-Wert sowie Anfärbeverhalten mit Sprühreagenzien als Auswahlkriterien zur Bewertung des Metabolitenspektrums eines Produzenten herangezogen werden. Hierbei werden meist Kombinationen unterschiedlicher Sprühreagenzien wie Anisaldehyd/Schwefelsäure (charakteristische Anfärbung von Glykosiden, Steroiden, Terpenen, Makroliden, Phenolen) und Ehrlich Reagenz (Amine, Indole, Furane, Pyrrole) verwendet. Dies dient dazu, eine möglichst große Zahl an Verbindungen sichtbar zu machen und erlaubt vielfach erste Rückschlüsse auf das Vorliegen bestimmter Substanzklassen. Eine exakte Identifizierung von bereits bekannten Metaboliten kann hier jedoch nicht erfolgen, was sich als einer der größten Nachteile dieser Form des chemischen Screenings erweist.

Die insbesondere in den letzten Jahrzehnten intensivierte Forschung auf dem Gebiet der Naturstoffisolierung hat mittlerweile nämlich dazu geführt, dass zunehmend häufig - bis zu über $90 \%$ bei terrestrischen Streptomyceten - bereits bekannte Verbindungen isoliert werden. Um ein effizientes Auffinden neuer Substanzen sicherzustellen, ist es daher unumgänglich, in einem möglichst frühen Stadium bereits bekannte Verbindungen zu erkennen und auszumustern. Dieser als Dereplikation bezeichnete Vorgang ist Gegenstand aktueller Forschung.

Die Kopplung von chromatographischen mit spektroskopischen Untersuchungsmethoden, wie z. B. LC-NMR, LC-CD, LC-UV/VIS, GC-EI-MS sowie LC-ESI-MS ermöglicht die Erstellung mehrdimensionaler Substanzdatenbanken und trägt damit erheblich zu einer Vereinfachung des Dereplikationsprozesses bei. Diese in den letzten Jahren entwickelten Methoden erlauben die Wiedererkennung von Komponenten komplexer Gemische ohne vorherige präparative Trennung, was drastische Zeit- und Kosteneinsparungen mit sich bringt. 
Da die LC-NMR-Kopplung auf relativ hohe Substanzmengen angewiesen sowie relativ teuer ist, kann sie nicht für Routineuntersuchungen eingesetzt werden. Als zusätzlicher Nachteil erweist sich die nicht immer vollständige Substanztrennung mittels HPLC, welche zu komplexen, nicht auswertbaren NMR-Spektren von Stoffgemischen führt. Ein HPLC-CD-basiertes Screening andererseits setzt das Vorhandensein chiraler Kohlenstoffatome sowie geeigneter chromophorer Gruppen in einem Molekül voraus, wobei sich nur wenige Informationen über die vorliegenden Substanzen aus den erhaltenen CD-Spektren ableiten lassen. Die mangelnde Spezifizität von UV-Spektren (mehrere Substanzen können ähnliche UV-Spektren besitzen), ihre Abhängigkeit vom jeweiligen pH-Wert und die Tatsache, dass viele Substanzen keine oder nur geringe UV-Absorption zeigen, macht auch ein HPLC-UV/VIS-basiertes Screening zu einem wenig zuverlässigen Ansatz. Der Einsatz der GC-MS-Kopplung birgt den Nachteil, dass nur thermisch stabile, flüchtige und zumeist unpolare Substanzen zugänglich sind. Die darin begründete Notwendigkeit einer nicht immer gelingenden Derivatisierung von ansonsten hier nicht detektierbaren Substanzen erschwert den Identifikationsprozess. Weiterhin wirkt sich bei dieser Methode die häufig beobachtete Bildung von Artefakten störend aus. ${ }^{27}$ Der Einsatz von HPLC-MS im Screeningsprozess schließlich bringt als Nachteil mit sich, dass die Messparameter Retentionszeit und Molekulargewicht in vielen Fällen nicht ausreichen, um eine Substanz eindeutig zu charakterisieren. Dies kann zu einem versehentlichen Aussortieren von noch unbekannten Verbindungen führen.

Aus den angegebenen Gründen wurde eine Methode weiterentwickelt, welche die umfassende, treffsichere und zugleich sensitive Analyse unterschiedlicher Verbindungsklassen durch gleichzeitige Aufnahme verschiedener spektroskopischer Daten gestattet. Hier erwies sich die Kopplung von HPLC und UV/VIS-DAD als sinnvoll, welche die parallele Messung eines UV-Chromatogramms ${ }^{*}$ und -Spektrums der einzelnen Verbindungen in einem Substanzgemisch ermöglicht. Durch Kopplung mit einem ESIMassenspektrometer können zusätzlich deren Molekulargewichte sowie mittels MS/MS das für viele Verbindungen charakteristische Fragmentierungsmuster ermittelt werden. Somit können während eines einzelnen Messvorgangs mehrere substanzspezifische Daten erhalten werden, welche in den meisten Fällen zur eindeutigen Identifizierung einer Verbindung ausreichen. Voraussetzung für diesen Ansatz ist der Zugang zu einer großen Zahl an Reinsubstanzen, die möglichst den Strukturraum der zu untersuchenden

\footnotetext{
* Gemeint ist ein Chromatogramm mit Detektion durch Messung der Extinktion.
} 
Naturstoffe abdecken sollten. Diese bilden ferner die Grundlage zur Erstellung einer umfassenden Substanzbibliothek.

Der Einsatz der HPLC-UV/VIS-MS/MS-Kopplung erfolgt dabei im Rahmen des chemischen Screenings und liefert ergänzende Informationen, welche über die Ergebnisse aus dünnschichtchromatographischen Untersuchungen und die Resultate aus biologischen Testungen hinausgehen. Produziert ein Stamm ausschließlich bereits bekannte Sekundärstoffe, können diese mittels Datenbankvergleich zugeordnet und das Metabolitenspektrum somit vollständig charakterisiert werden. Weiterhin ist ein Einsatz der HPLC-UV/VIS-ESIMS/MS-Kopplung zur raschen Identifizierung von gesuchten neuen bzw. bereits bekannten Verbindungen während des Isolierungsprozesses mitunter gewinnbringend (Abbildung 1). Ebenso ist diese Screeningsmethode für eine effiziente Identifizierung von zu untersuchenden Verbindungen im Rahmen von Fütterungs- und Medienvariationsansätzen geeignet.

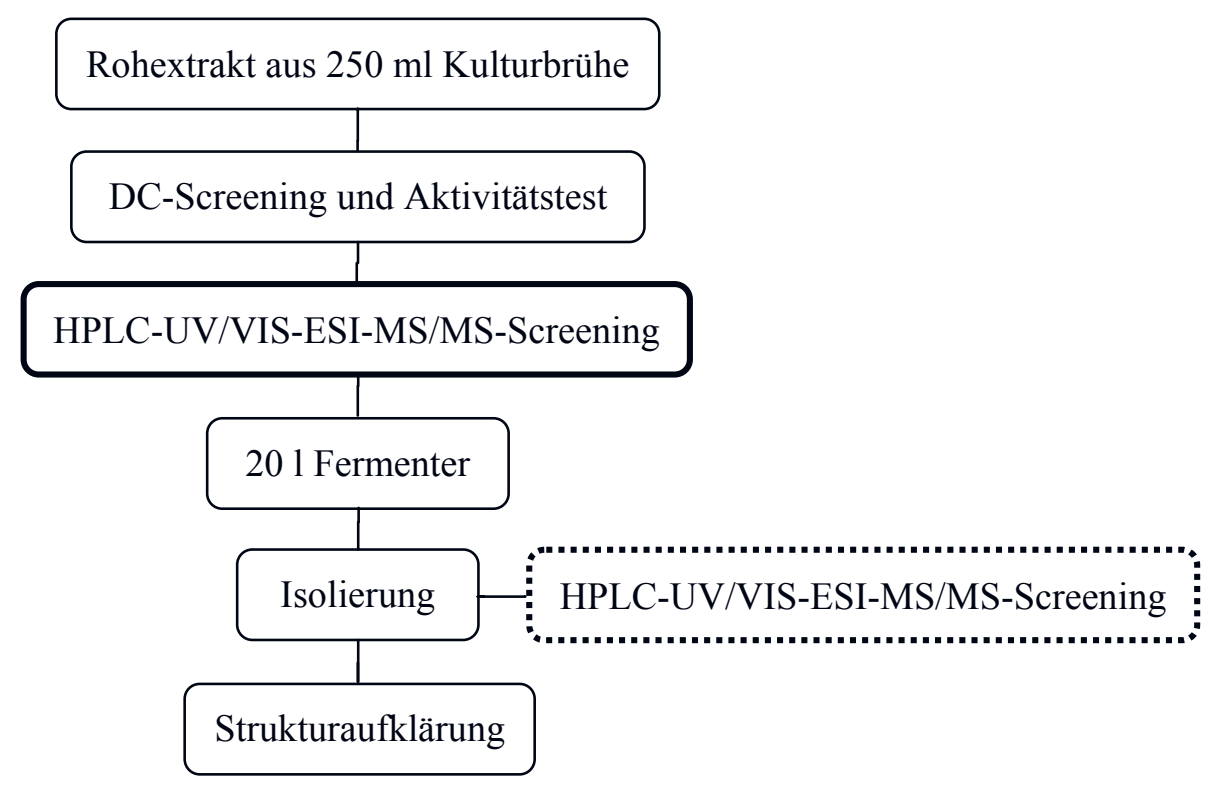

Abbildung 1 Vorgang der Naturstoffisolierung aus Mikroorganismen. 


\section{HPLC-UV-ESI-MS/MS-Datenbank}

\subsection{Aufbau einer HPLC-UV-ESI-MS/MS-Datenbank}

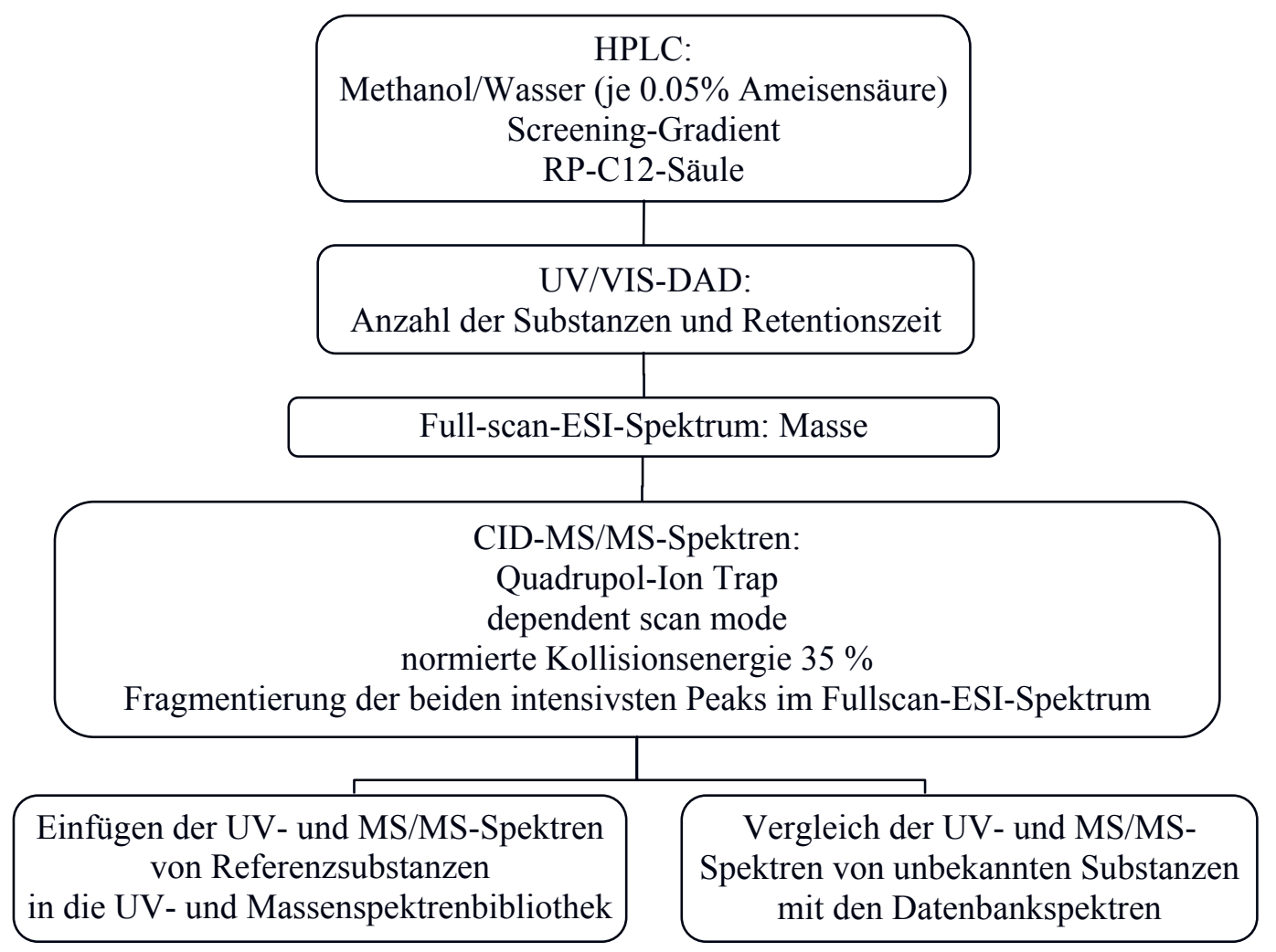

Abbildung 2 Schematischer Verlauf der Messung.

\subsubsection{HPLC-Bedingungen}

Für die chromatographische Trennung wurden zwei HPLC-Säulen mit unterschiedlichen Säulenmaterialien und -größen von zwei verschiedenen Herstellern zu Vergleichzwecken herangezogen. Bei gleichem Durchmesser von zwei Millimetern besaß die RP-C18Kieselgelsäule (Macherey \& Nagel) eine Länge von 125 mm, während eine mit RP-C12Material befüllte Säule (Phenomenex) $150 \mathrm{~mm}$ lang war. Aufgrund der geringeren Zahl freier Silanolgruppen und der sich daraus ergebenden besseren Trenneigenschaften, insbesondere für basische Verbindungen, wurde letztere für die Erstellung der Spektrenbibliothek ausgewählt. Als mobile Phase diente ein Methanol/Wasser-Gradient (Abbildung 3), welcher zur Erhöhung der Trennschärfe jeweils mit $0.05 \%$ Ameisensäure versetzt wurde. Ohne Säurezusatz war die Tendenz zu einer Peakverbreiterung erkennbar, was die 
automatisierte Fragmentierung der gewünschten Verbindung im dependent scan mode* erschwerte (siehe Kapitel 4.1.4). Vergleichende Untersuchungen mit dem Laufmittelsystem Acetonitril/Wasser führten nicht zu generell besseren Ergebnissen, weswegen dies aufgrund schlechterer Lösungseigenschaften bei der Probenvorbereitung nicht zum Einsatz kam. Eine Flussrate von $300 \mu 1 /$ min sowie ein Injektionsvolumen von $5 \mu$ erwiesen sich für die angestrebten Analysen als optimale Messparameter. Die HPLC-chromatographischen Trennungen fanden durchweg bei Raumtemperatur statt.

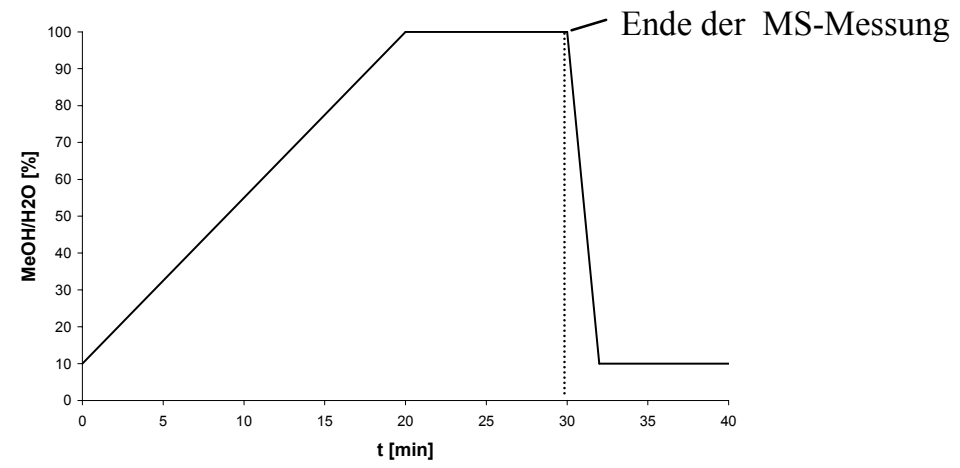

Abbildung 3 Verlauf des zur Erstellung der HPLC-UV-ESI-MS/MS-Datenbank verwendeten Methanol/Wasser-Gradienten.

\subsubsection{UV/VIS-Diode-Array-Detektor}

Die per HPLC getrennten Substanzen wurden mittels eines UV/VIS-DAD im Bereich von 200 bis $800 \mathrm{~nm}$ bei einer Auflösung von $5 \mathrm{~nm}$ spektroskopisch analysiert. Anhand des so erhaltenen UV-Chromatogramms konnte die Anzahl der in der Probe vorhandenen Verbindungen abgeschätzt werden. Die dadurch ebenfalls zugänglichen UV-Spektren beinhalteten zusätzlich oft wertvolle Informationen über die vorliegenden Substanzklassen. Verbindungen, welche in dem verwendeten UV/VIS-Messbereich keine oder nur geringe Absorptionseigenschaften besaßen, konnten hier jedoch nicht detektiert werden.

\subsubsection{Electrospray-Massenspektrometrie}

Bei dem zum Aufbau der Datenbank eingesetzten Messaufbau war das Massenspektrometer in Serie an den UV/VIS-Detektor gekoppelt. Dadurch ergab sich eine

* Automatischer Messablauf, in dem durch die Voreinstellungen nur bestimmte Ionensignale fragmentiert werden. 
Zeitverzögerung von etwa fünf Sekunden zwischen UV/VIS- und MS-Signal. Aufgrund des verwendeten flüssigchromatographischen Trennverfahrens war der Einsatz von ESI (Electrospray Ionisation) als Ionisierungsart vorgegeben. Diese schonende Methode erwies sich bei der Analyse der vorliegenden Naturstoffe als durchaus vorteilhaft, da sie in den meisten Fällen die Bildung eines Molekülions gewährleistete. Um trotz der vorgegebenen hohen Flussrate die Erzeugung eines optimalen Sprays mit möglichst geringen Durchmessern zu erreichen, wurde eine erhöhte Kapillartemperatur von $220{ }^{\circ} \mathrm{C}$ gewählt.

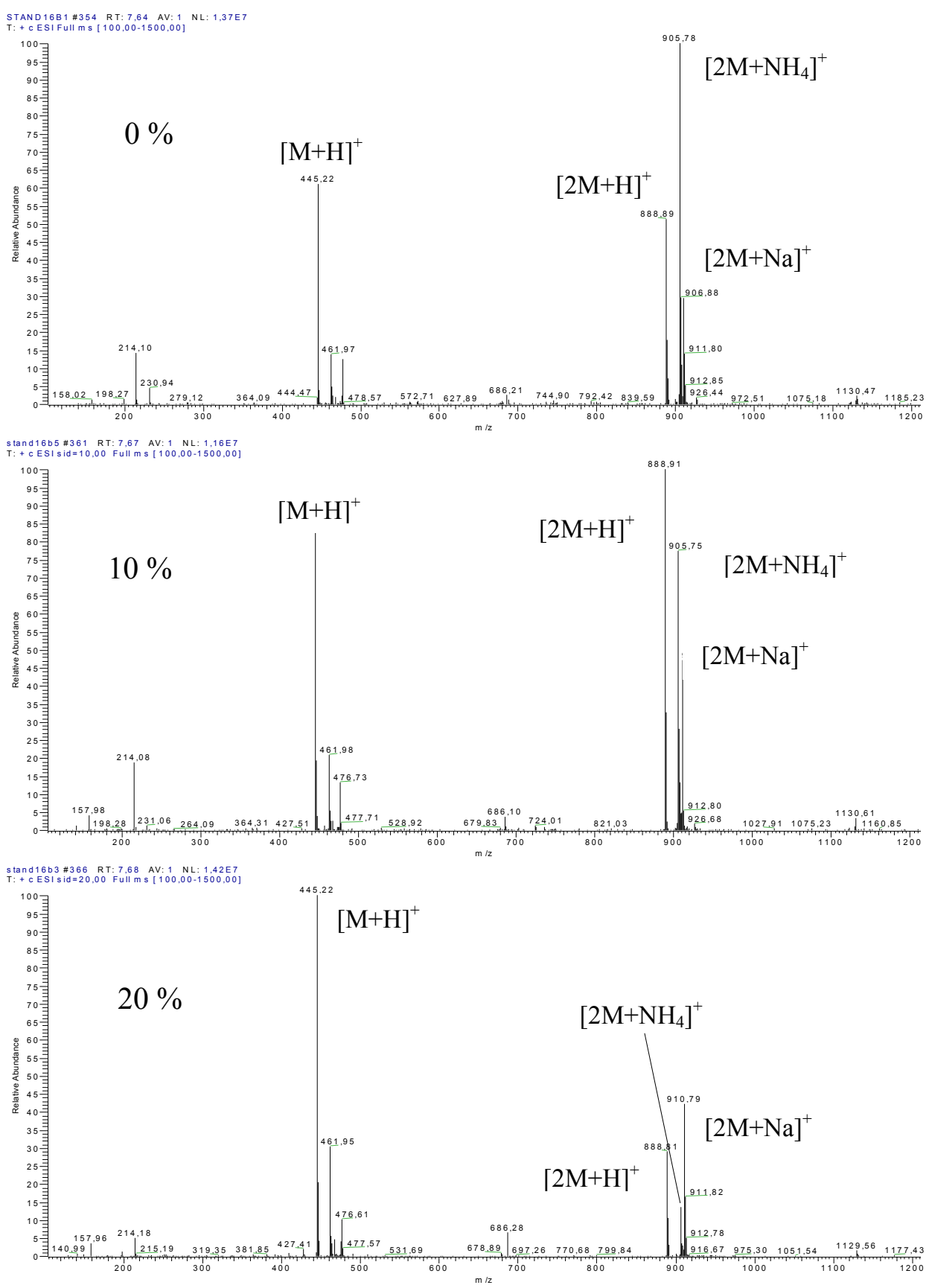

Abbildung 4 Mit unterschiedlichen Kollisionsenergien (ISCID) erhaltene MS-Spektren. 
Die Massendetektion wurde im positiven und im negativen Modus durchgeführt, wobei es in den meisten Fällen zur Bildung von Monomer-Ionen ([M+H $]^{+}-,[\mathrm{M}+\mathrm{Na}]^{+}-$und $\left.[\mathrm{M}-\mathrm{H}]^{-}\right)$ sowie Dimer-Ionen $\left([2 \mathrm{M}+\mathrm{H}]^{+}-,[2 \mathrm{M}+\mathrm{Na}]^{+}\right.$- und $\left.[2 \mathrm{M}-\mathrm{H}]^{-}\right)$kam. Die häufig in den Massenspektren beobachteten Dimer-Ionen führten $\mathrm{zu}$ keinem interpretierbaren Fragmentierungsmuster und störten zudem aufgrund ihrer hohen Intensitäten eine automatisierte Selektion der zu fragmentierenden Ionen (siehe Kapitel 4.1.4). Somit war es zweckmäßig, die Dimerbildung zu unterdrücken und damit eine höhere Signalintensität der Monomerionen $\mathrm{zu}$ gewährleisten. Um dies $\mathrm{zu}$ ermöglichen, musste eine zusätzliche Kollisionsenergie in der Quelle (In Source Collision-induced Dissociation, ISCID) angelegt werden, so dass auftretende Dimere zurück in die Monomeren zerfielen, wobei letztere wiederum nicht zur Fragmentbildung angeregt werden durften. Aufgrund der unterschiedlichen Stabilität der Moleküle musste eine mittlere Energie dafür ausgewählt werden. Anhand von Experimenten mit verschiedenen Energien zeigte sich, dass ein Wert von $10 \mathrm{~V}$ für die durchgeführten Untersuchungen am besten geeignet ist (Abbildung 4).

Datenbanken, welche als Moleküleigenschaften die Retentionszeit, das UV-Spektrum sowie das Molekulargewicht beinhalten, sind bereits weit verbreitet und werden vielfach eingesetzt. Konstitutionsisomere ohne charakteristische UV-Spektren können anhand dieser Daten allerdings nicht unterschieden werden. Isomere Verbindungen, welche iden-

tische Chromophor-Grundgerüste, aber unterschiedliche Seitenkette besitzen, weisen gleiche UV- und Massenspektren sowie ähnliche Retentionszeiten auf und sind somit ebenfalls nur schwer zu identifizieren. Aus diesen Gründen liegt die Einbeziehung zusätzlicher Informationen in eine Datenbank nahe, welche die Unterscheidung solcher Verbindungen ermöglicht. In der vorliegenden Arbeit wurde diesbezüglich auf MS/MS-spektrometrische Daten zurückgegriffen.

\subsubsection{Die ESI-MS/MS-Methode}

Die bisher vorhandenen MS/MS-Techniken basieren auf einer durch Zusammenstöße mit Argon- bzw. Heliumatomen induzierten Fragmentierung (Collision-induced Dissociation, CID). Diese wird bei Triple-Quadrupol- und Ion-trap-Massenspektrometern in einem separaten Prozess vollzogen. ${ }^{28}$ Bei Einsatz der beiden letztgenannten Instrumente findet somit eine Vorauswahl der $\mathrm{zu}$ fragmentierenden Massenionen statt, was $\mathrm{zu}$ geringeren Störsignalen und demgemäß zu einer deutlich höheren Qualität der CID-Spektren führt. Im 
Zuge dieser Arbeit wurde auf die Verwendung eines Ion-Trap-Massenspektrometers zurückgegriffen.
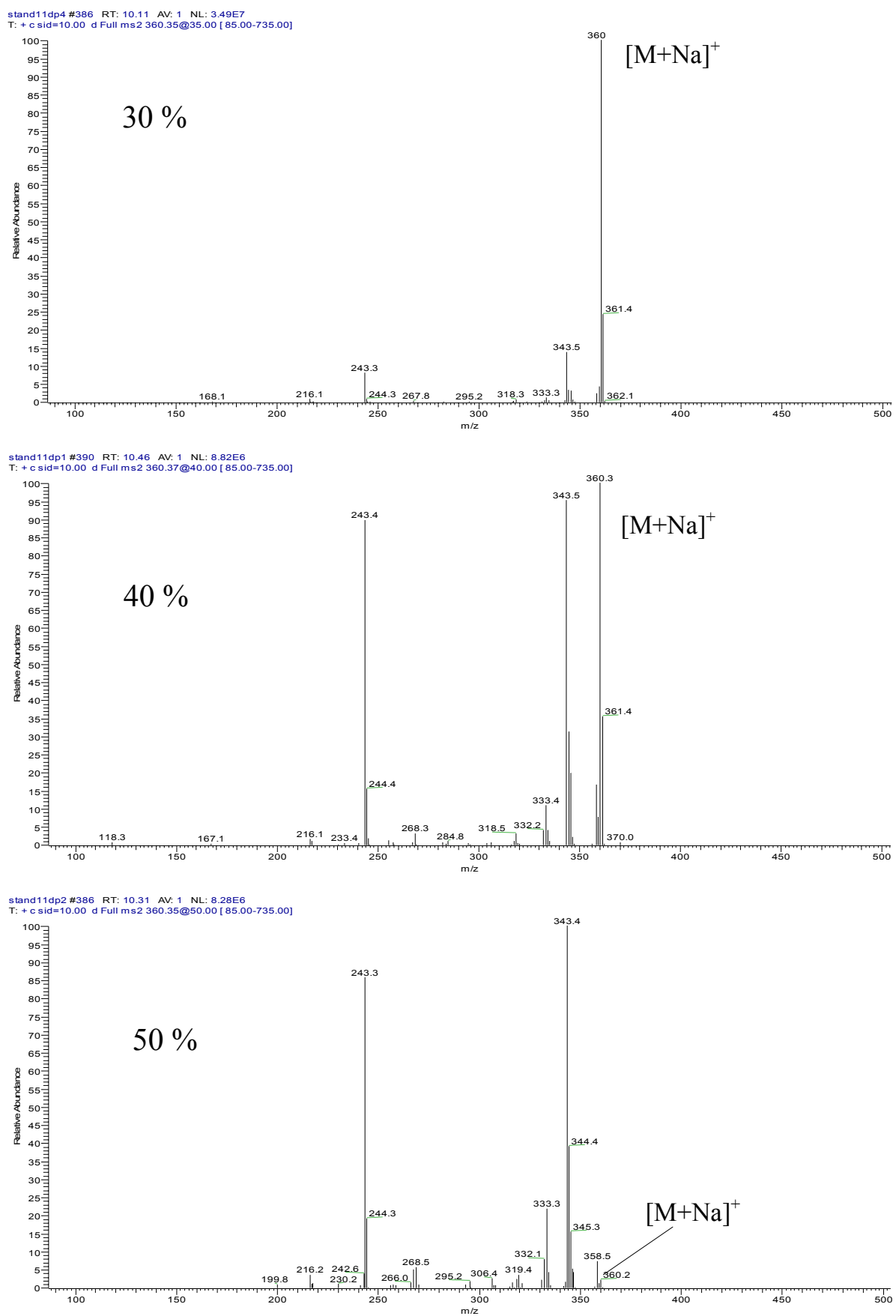

Abbildung 5 Mit unterschiedlichen Kollisionsenergien erhaltene MS/MS-Spektren.

Die zur Induktion der Fragmentierung erforderliche Kollisionsenergie nimmt mit steigenden $\mathrm{m} / \mathrm{z}$-Werten $\mathrm{zu}^{29} \mathrm{Um}$ eine bessere Vergleichbarkeit zu ermöglichen, werden in der Regel normierte Kollisionsenergien angegeben, welche um den masseabhängigen sowie um einen gerätespezifischen Teil korrigiert sind. Wählt man für das Experiment zu 
niedrige Kollisionsenergien, so kommt es zu keiner ausreichenden Fragmentierung, während zu hohe Energien niedrigere Fragmentintensitäten zur Folge haben (Abbildung 5). Um den für die Erstellung einer Naturstoff-Datenbank geeigneten Energiewert zu ermitteln, wurden im Vorfeld systematische Untersuchungen durchgeführt. Diese führten zu dem Ergebnis, dass eine mittlere Kollisionsenergie von $35 \%$ zur Fragmentierung der vorliegenden, strukturell hoch variablen Verbindungsklassen am zweckmäßigsten erschien. Fragmentierungen erfolgen im Regelfall an der Stelle des Moleküls, an der die positive bzw. negative Ladung lokalisiert ist. Seltener kommt es zur sogenannten „charge-remote“Fragmentierung, welche unabhängig von der Position der Ladung ist. In diesem Zusammenhang ist auffällig, dass protoniert vorliegende Molekülionen im Vergleich zu entsprechenden Natriumaddukten über deutlich unterschiedliche Fragmentierungsmuster verfügen. Dies kann dadurch erklärt werden, dass $\mathrm{H}^{+}$- und $\mathrm{Na}^{+}$-Ionen an unterschiedlichen Stellen des zu fragmentierenden Moleküls binden und damit auch die Ladung an verschiedenen Positionen eingeführt wird. Zusätzlich findet eine Wanderung der $\mathrm{H}^{+}$-Ionen innerhalb eines Moleküls während des Fragmentierungsprozesses statt.

Die Selektion der Massenionen, welche fragmentiert werden sollten, erfolgte im dependent scan Modus. Bei der vorliegenden Arbeit wurden die zugrunde liegenden Parameter so gewählt, dass es zur sukzessiven Fragmentierung der beiden intensivsten Ionen im Fullscan-Spektrum* kam.

Die Fragmentierung des zweit-intensivsten Ions im dependent scan Modus wurde aus folgenden Gründen durchgeführt:

1. $[\mathrm{M}+\mathrm{H}]^{+}$- und $[\mathrm{M}+\mathrm{Na}]^{+}$-Ionen einer Substanz weisen unterschiedliche Fragmentierungsmuster und Intensitäten auf. Im Fall des cyclischen Peptides F-36 (13) konnten die Peaks der beiden Addukt-Ionen mit deutlich unterschiedlichen Intensitäten detektiert werden (Abbildung 6). Das $[\mathrm{M}+\mathrm{Na}]^{+}$-Ion wies hier eine höhere Intensität im MS-Spektrum auf als das $[\mathrm{M}+\mathrm{H}]^{+}$-Ion. Die regelmäßige cyclische Anordnung der Carbonylsauerstoffatome begünstigt die Bildung des Natriumadduktes. Die anschließende Fragmentierung dieses Ions wiederum lieferte ein MS/MS-Spektrum mit geringeren Fragmenten (Abbildung 7).

\footnotetext{
* Das direkt nach ES-Ionisierung aufgenommene Massenspektrum.
} 


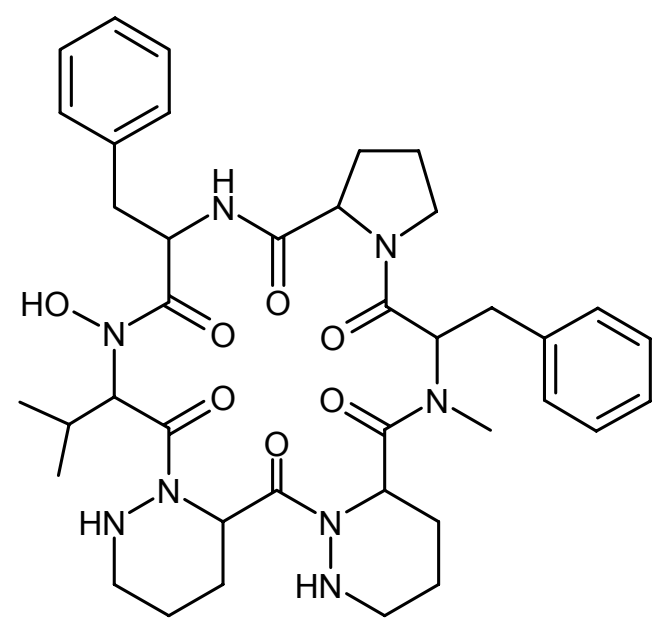

13

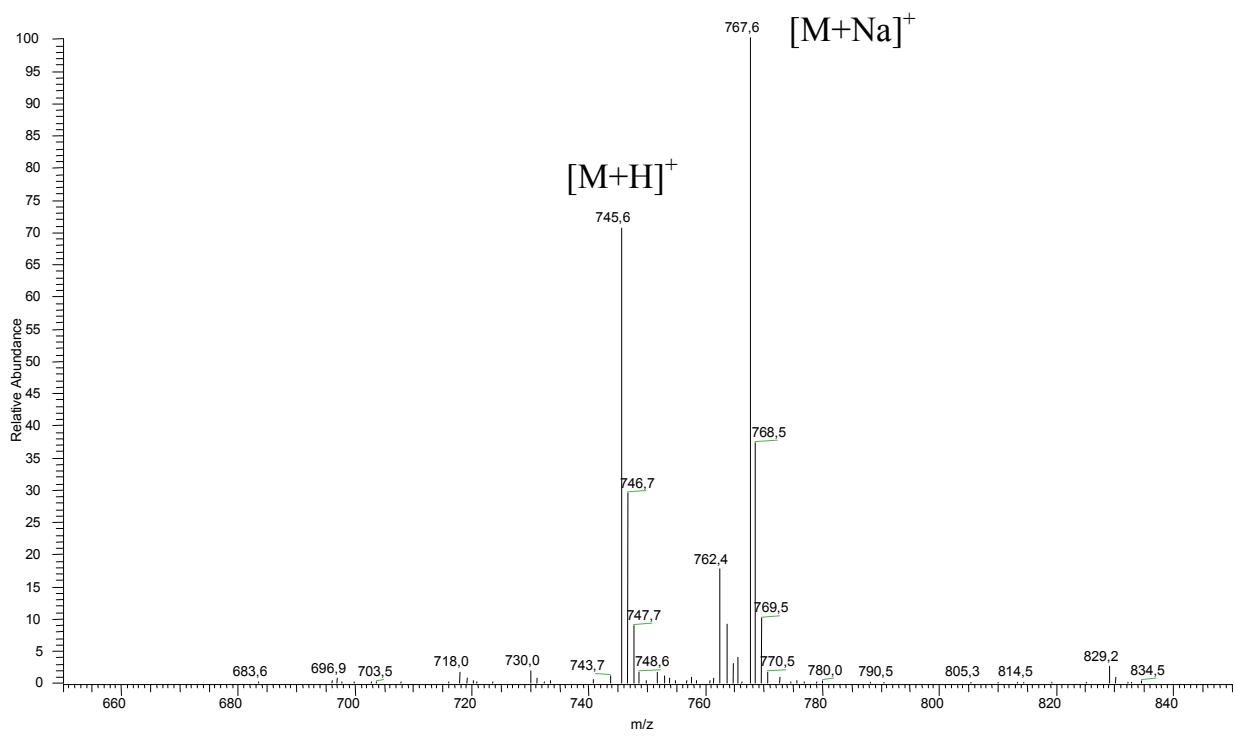

Abbildung 6 ESI-Massenspektrum von F-36 (13) (mit unterschiedlichen Intensitäten des $[\mathrm{M}+\mathrm{H}]^{+}$- und $[\mathrm{M}+\mathrm{Na}]^{+}$-Ions).
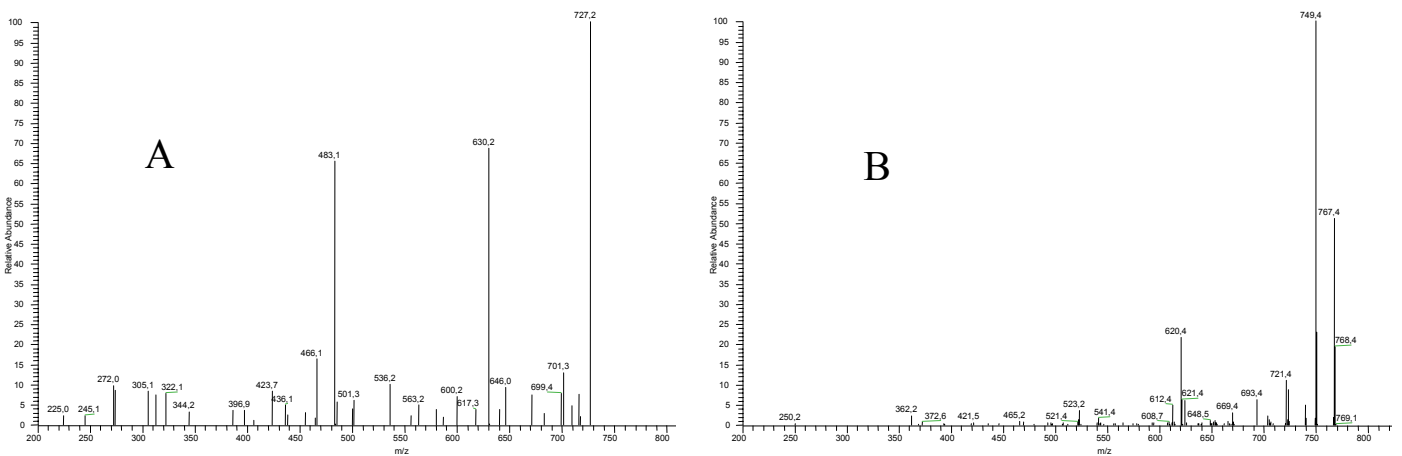

Abbildung 7 ESI-MS/MS-Spektrum des $[\mathrm{M}+\mathrm{H}]^{+}-(\mathrm{A})$ und des $[\mathrm{M}+\mathrm{Na}]^{+}-$Ions (B) von 13. 
2. In manchen Fällen ist die Intensität des Dimer-Peaks höher als die des MonomerIons. Da ersterer meist kein interpretierbares Fragmentierungsmuster liefert, ist man zur Erstellung eines aussagekräftigen MS/MS-Spektrums hier auf das zweitintensivste Ion angewiesen. In Abbildung 8 ist das Massenspektrum von Carquinostatin A (14) als Beispiel angeführt. Im Falle dieser Verbindung weist das $[2 \mathrm{M}+\mathrm{Na}]^{+}$-Ion etwa doppelte Intensität im Vergleich mit dem $[\mathrm{M}+\mathrm{H}]^{+}$-Ion auf.<smiles>CC(C)=CCc1ccc2[nH]c3c(c2c1)C(=O)C(=O)C(C)=C3C[C@H](C)O</smiles>

14

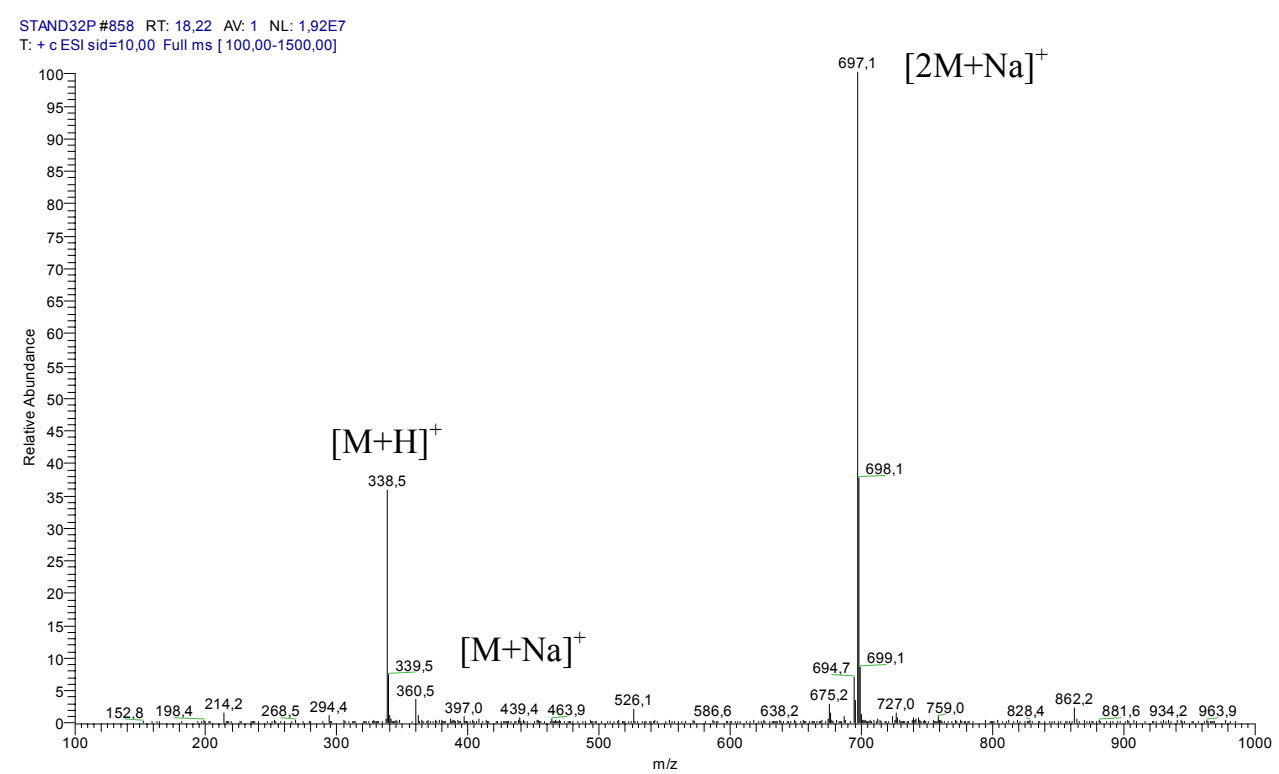

Abbildung 8 ESI-Massenspektrum von Carquinostatin A (14) mit unterschiedlichen Intensitäten der $[\mathrm{M}+\mathrm{H}]^{+}-,[\mathrm{M}+\mathrm{Na}]^{+}$- und $[2 \mathrm{M}+\mathrm{Na}]^{+}$-Ionen.

3. Die Intensität der Peaks von Hintergrund- und überlappenden Ionen ist in einigen Fällen größer als die der untersuchten Ionen. Dies ist teilweise auf Verunreinigungen aus dem System zurückzuführen. Die Trennung konnte durch Zusatz von Ameisensäure in den Lösungsmitteln verbessert werden. ${ }^{*}$ In Abbildung 9 ist das Massenspektrum eines Gemisches von Phthalsäureester und einer zu unter-

\footnotetext{
* Keine Verwendung von TFA, da diese Säure sehr gut ionisierbar ist und im negativen Modus zu einer hohen Zahl an Untergrundsignalen führt.
} 
suchenden Substanz wiedergegeben. Phthalsäureester sind als Weichmacher in Schläuchen weit verbreitet und wurden aufgrund ihrer hohen Empfindlichkeit bei jeder Messung detektiert. $[\mathrm{M}+\mathrm{H}]^{+}$- und $[2 \mathrm{M}+\mathrm{Na}]^{+}$-Ionen zeigen hohe PeakIntensitäten bei einer Retentionszeit zwischen 23 und 24 Minuten. Besitzt die interessierende Substanz eine vergleichbare Retentionszeit, musste in zahlreichen Fällen von einer Überlagerung der Massenionen ausgegangen werden. In Abbildung 9 ist zur Verdeutlichung ein MS-Spektrum dargestellt, in welchem das Ion der untersuchten Verbindung $\left(\mathrm{m} / \mathrm{z} 815,\left[\mathrm{M}_{2}+\mathrm{Na}\right]^{+}\right)$eine geringere Intensität als das Dimer-Ion von Phthalsäureester $\left(m / z\right.$ 803, $\left.\left[2 \mathrm{M}_{1}+\mathrm{Na}\right]^{+}\right)$aufweist.

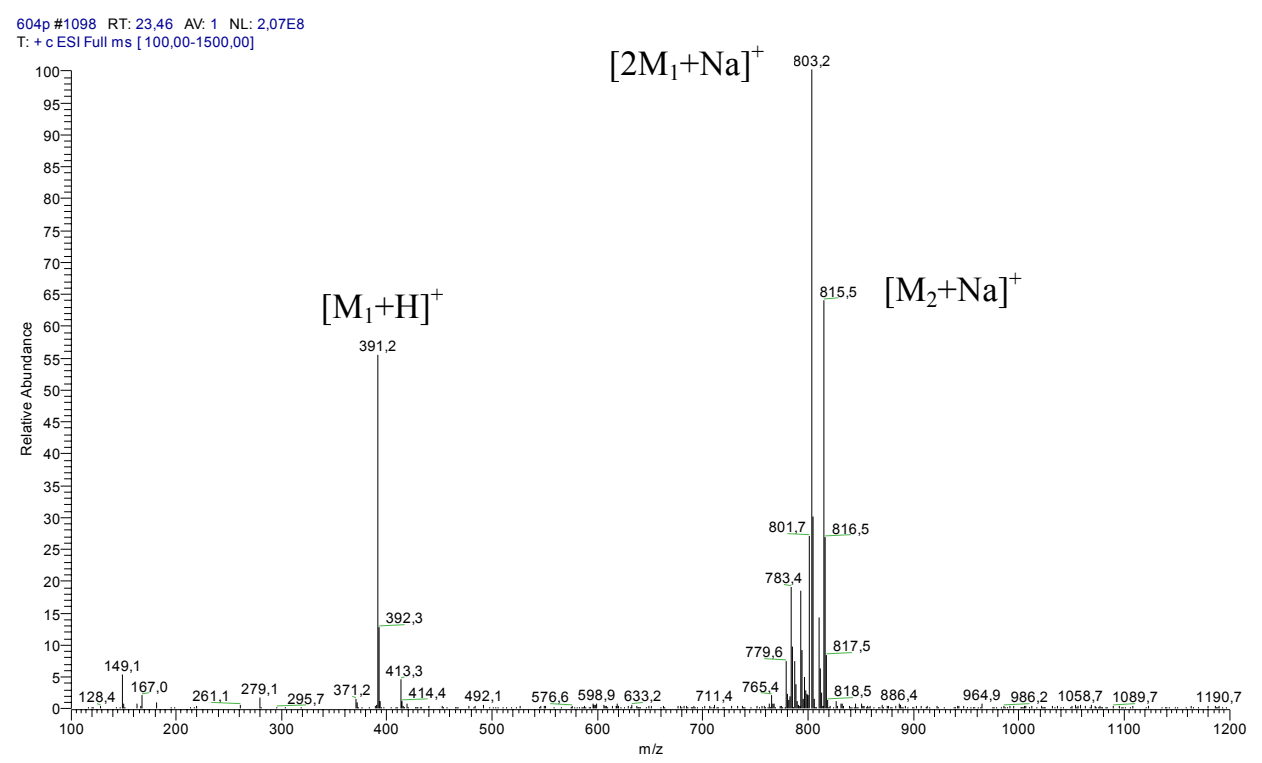

Abbildung 9 ESI-Massenspektrum eines Gemisches von Phthalester $\left(\mathrm{M}_{1}\right)$ und einer zu untersuchenden Substanz $\left(\mathrm{M}_{2}\right)$.

Als problematisch erwies sich jedoch nach wie vor die Trennung homologer Verbindungen. Eine Überlappung der Quasimolekülionen mit unterschiedlichen Intensitäten war auch bei den schwer zu trennenden Actinomycinen häufig anzutreffen. Abbildung 10 zeigt das Massenspektrum eines Gemisches von zwei Actinomycinen, welche über eine Massendifferenz von zwei Einheiten verfügen. Actinomycin $\mathrm{C}_{2}$ weist ein $[\mathrm{M}+\mathrm{H}]^{+}$-Ion bei $\mathrm{m} / \mathrm{z} 1270$ und ein $[\mathrm{M}+\mathrm{Na}]^{+}$-Ion bei $m / z 1292$ auf, während Actinomycin $\mathrm{X}_{0 \beta}$ ein $[\mathrm{M}+\mathrm{H}]^{+}$-Ion bei $m / z 1272$ sowie ein $[\mathrm{M}+\mathrm{Na}]^{+}$-Ion bei $m / z 1294$ zeigt. Da die $[\mathrm{M}+\mathrm{Na}]^{+}$-Ionen der Actinomycine ein spezifischeres und intensiveres Fragmentierungsmuster ergaben, wurde deren Fragmentierung bei der Erstellung der Datenbank bevorzugt. Eine Fragmentierung beider Substanzen im dependent scan Modus setzte die automatische Selek- 
tion des zweitintensivsten Ions voraus. Diese war ebenso unumgänglich, wenn lediglich die Fragmentierung der Substanz mit der geringeren Quasimolekülionenintensität das Ziel war.

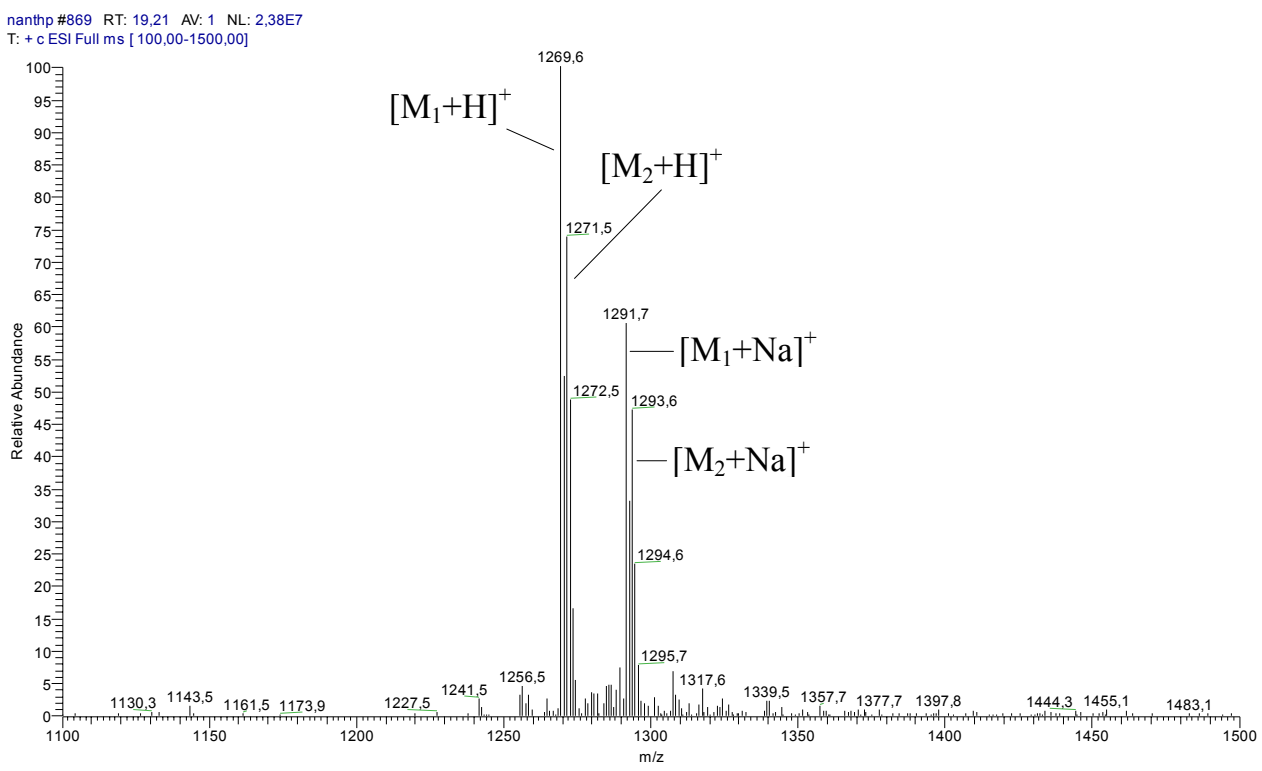

Abbildung 10 ESI-Massenpektrum eines Gemisches von Actinomycin $\mathrm{C}_{2}\left(\mathrm{M}_{1}\right)$ und Actinomycin $\mathrm{X}_{0 \beta}\left(\mathrm{M}_{2}\right)$.

\subsubsection{UV/VIS-MS/MS-Bibliotheken}

Zur Erstellung der Datenbank wurde ein Programm benötigt, welches die Eintragung der UV- und MS/MS-Spektren sowie weiterer Informationen über die untersuchten Verbindungen, wie Retentionszeit, Substanzname, Struktur und Summenformel, erlaubt. Im Rahmen dieser Arbeit wurden zwei für diesen Zweck geeignete Softwarelösungen verglichen.

\section{NIST Algorithmus}

In der Software Xcalibur werden MS/MS-Massenspektren automatisch in das von der Datenbank NIST benutzte Dateiformat umgewandelt. Letztere wurde ursprünglich für EIMS-Spektren entwickelt, woraus sich einige Beschränkungen bei der Verwendung von ESI-MS/MS-Spektren ergeben. So ist es beispielsweise nicht möglich, zusätzliche Informationen wie Retentionszeiten oder die verwendete Messmethode einzubeziehen.

Sind andere spektroskopische Daten wie z. B. UV/VIS-Spektren zugänglich, können diese ebenfalls nicht ergänzend in die zu erstellende Datenbank auf der Basis von NIST einge- 
tragen werden. An dieser Stelle wird ein zusätzliches Programm für die Erstellung der UV/VIS-Datenbank benötigt. Bisher ist ein derartiges Modul, welches die Kopplung mit der NIST-Datenbank ermöglicht, aber nicht kommerziell verfügbar. Das führt dazu, dass Totalionenstrom- und UV/VIS-Chromatogramm unabhängig voneinander in unterschiedlichen Datenbanken bzw. Programmen bearbeitet werden müssen. Dies erschwert die Durchführung einer Datenbanksuche und kann unter Umständen zu einer falschen Interpretation der Peaks führen, weswegen auf die Verwendung dieser Software verzichtet wurde.

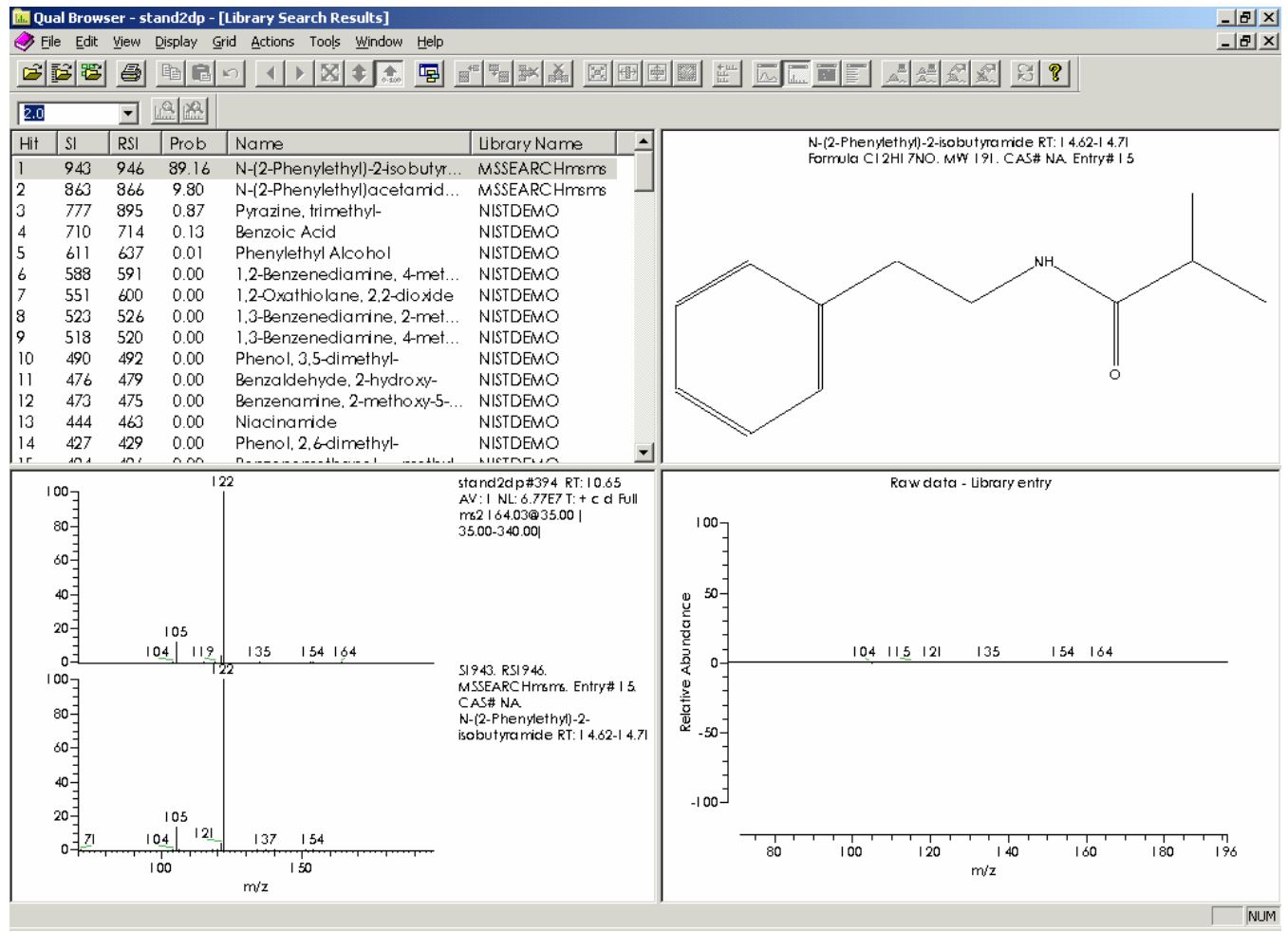

Abbildung 11 Eine auf dem NIST-Algorithmus basierende Datenbank.

\section{MS-Manager von ACD-Labs}

Dieses Programm eröffnet verschiedene Bearbeitungsmöglichkeiten. So kann es nicht nur zur Erstellung einer Datenbank dienen, es ermöglicht ebenfalls eine Bearbeitung der erhaltenen Massenspektren. Der größte Vorteil dieses Programms liegt darin, dass mehrere spektroskopische Daten, wie MS/MS-, UV-, IR- sowie NMR-Daten, hier miteinander gekoppelt werden können. Ebenso besteht die Möglichkeit, die im positiven wie die im negativen Modus erhaltenen MS/MS-Spektren zu kombinieren. Ergänzend können zusätzliche Informationen wie Name und Struktur einer Verbindung, deren Summenfor- 
mel, Retentionszeit sowie die verwendete Messmethode einbezogen werden. Die Eintragung der beiden letztgenannten Parameter erfolgt dabei automatisch. Eine Datenbanksuche mit diesem Programm erlaubt durch die vielseitige Verknüpfung verschiedener Optionen die gezielte Ermittlung der gewünschten Informationen. Bezieht man unterschiedliche spektroskopische Informationen in die Datenbank ein, können diese kombiniert und so ein effizienteres Auffinden der gesuchten Struktur ermöglicht werden. Das Importieren der MS-Spektren aus Xcalibur in den MS-Manager ist jedoch recht zeitaufwendig.

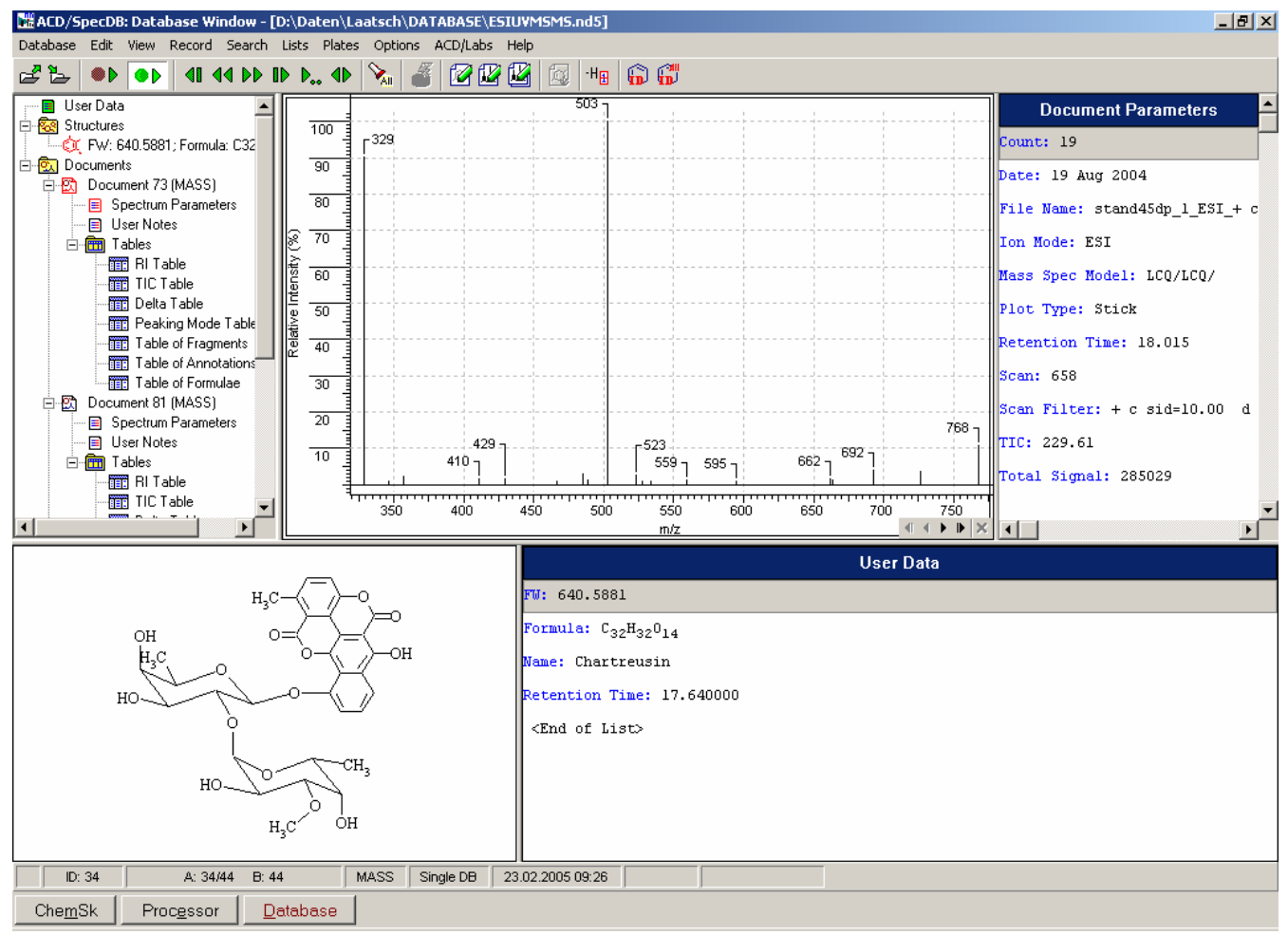

Abbildung 12 Eine im MS-Manager geöffnetes HPLC-UV-MS/MS-Datenbankfenster.

\subsection{Methodenanalyse}

Die erstellte HPLC-UV-ESI-MS/MS-Datenbank basiert auf in unserer Arbeitsgruppe isolierten bakteriellen Naturstoffen und beinhaltet bisher etwa 250 Verbindungen. Durch die Kopplung von HPLC, UV/VIS-DAD und Massenspektrometer sowie die induzierte Fragmentierung konnten die zu untersuchenden Substanzen getrennt und gleichzeitig deren Retentionszeiten, UV-Spektren, Massenzahlen sowie Fragmentierungsmuster ermittelt werden. Die in Abhängigkeit von den jeweiligen Moleküleigenschaften entstandenen $[\mathrm{M}+\mathrm{H}]^{+}$- und $[\mathrm{M}+\mathrm{Na}]^{+}$-Ionen führten ebenso zu unterschiedlichen, spezifischen Fragment- 
ierungsmustern. Sofern es die Peakintensitäten erlaubten, wurden beide MS/MS-Spektren in die Datenbank eingetragen.

Die Erstellung der Datenbank war mit einem hohen Zeit- und Arbeitsaufwand verbunden, da zunächst mehrere Experimente durchgeführt werden mussten, um optimale Messparameter und -bedingungen für die zu untersuchenden Substanzen zu ermitteln. Anschließend wurden die einzelnen Verbindungen untersucht und die erhaltenen UV- und Fragmentierungsspektren sowie weitere erforderliche Daten in die Datenbank eingetragen. Der vergleichsweise hohe Aufwand ist jedoch insofern gerechtfertigt, als es sich hier um eine Datenbank mit sehr hohem Informationsgehalt handelt, welche die effiziente und zweifelsfreie Identifikation von Verbindungen gewährleistet.

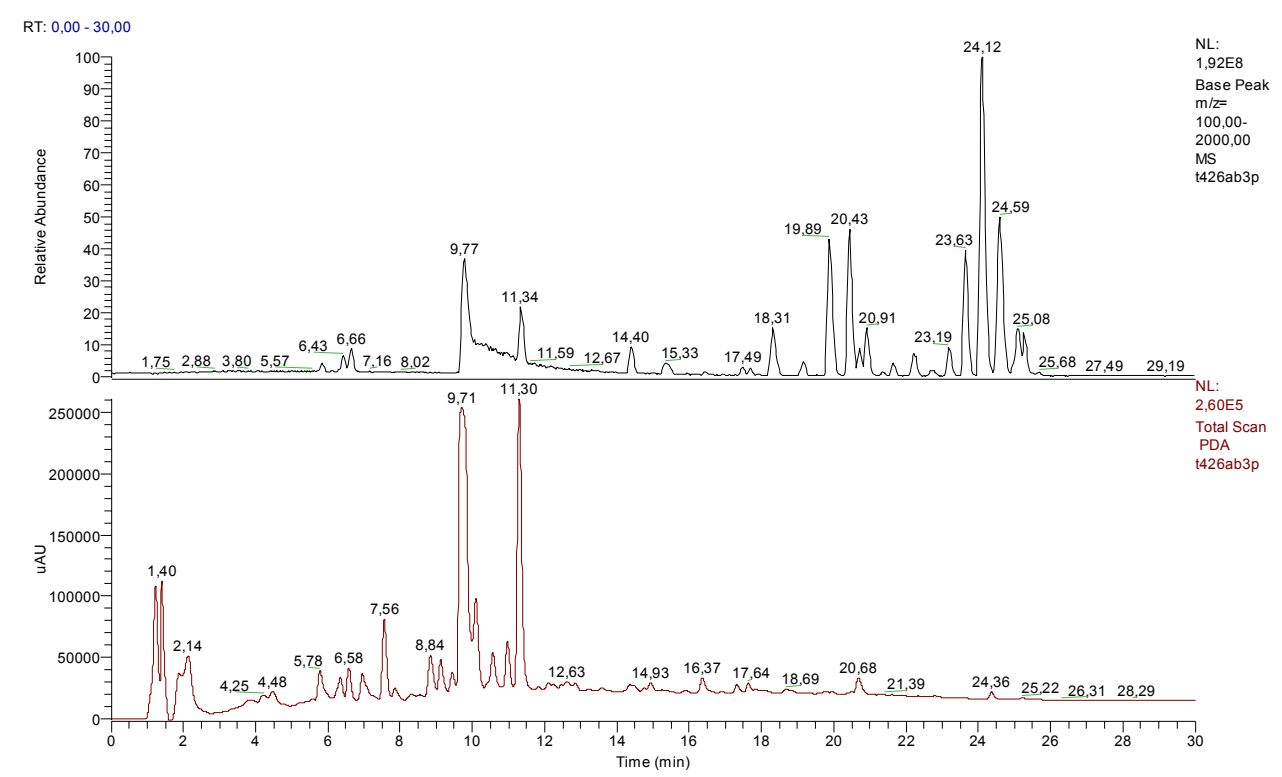

Abbildung 13 Ein Vergleich des Base-Peak- (oben) und UV/VIS-Chromatogramms (unten) eines Rohextraktes.

Sowohl Rohextrakte als auch durch chromatographische Trennung erhaltene Fraktionen wurden unter Verwendung identischer Bedingungen gemessen. Die Kombination der erhaltenen Daten führte in den meisten Fällen zu einer raschen Identifizierung der einzelnen Substanzen mit Hilfe der HPLC-UV-ESI-MS/MS-Datenbank. Darin gespeicherte Verbindungen wurden mit einer 100proz. Trefferausbeute wiedererkannt. Soll die Anzahl der in einer Probe enthaltenen Verbindungen abgeschätzt werden, muss darauf geachtet werden, dass nicht oder nur schwer ionisierbare Moleküle nicht unmittelbar im Base-PeakChromatogramm detektierbar sind. In derartigen Fällen ist jedoch die zusätzliche Verfügbarkeit des UV-Chromatogramms als ergänzende Informationsquelle häufig hilfreich 
(Abbildung 13). Umgekehrt können UV-inaktive Verbindungen vielfach im Ionenstromchromatogramm nachgewiesen werden. Liegt eine Substanz vor, welche nicht in der Datenbank enthalten ist, wurden Molekulargewicht und UV/VIS-Daten als Ausgangspunkt für eine weitere Suche in AntiBase ${ }^{30}$ bzw. Chapmann \& Hall ${ }^{31}$ mit dem Ziel der Strukturzuordnung durchgeführt.

Kann eine Substanz nicht identifiziert werden, bestehen folgende Möglichkeiten:

- Es handelt sich um eine bislang unbekannte Verbindung.

- Die Substanz ist zwar bekannt, aber nicht in der Datenbank enthalten.

- Die Substanz kann mittels ESI nicht oder nur schwer ionisiert werden, so dass kein Massenspektrum zugänglich ist.

- Die Peak-Intensität des Dimers bzw. deren Isotope sind trotz der verwendeten Kollisionsenergie (ISCID) wesentlich höher als die des Monomers.

- Es erfolgt keine vollständige Trennung per HPLC, so dass mehrere überlappende Ionen mit unterschiedlichen Intensitäten im Massenspektrum zu erkennen sind.

- Die in der Probe enthaltene Substanzmenge ist zu gering, so dass die Intensität des Quasimolekülions im Vergleich mit dem Hintergrund nur sehr niedrig ist.

Abbildung 14 zeigt das Beispiel einer Strukturidentifizierung im Rohextrakt des Stammes Alteromonas sp. T268. Die von den einzelnen Substanzen ermittelten MS/MS-Spektren wurden zur Suche in der HPLC-UV-ESI-MS/MS-Datenbank herangezogen, was zu den häufig von Bakterien produzierten Diketopiperazinen führte. Ein zusätzlicher Vergleich der Retentionszeiten und UV-Spektren bestätigte die Zuordnung. In diesem Zusammenhang wurde festgestellt, dass die vorhandenen Diastereomeren anhand des Fragmentierungsmusters nicht unterschieden werden können. Dies gelingt in vielen Fällen jedoch über einen Vergleich der Retentionszeiten. 

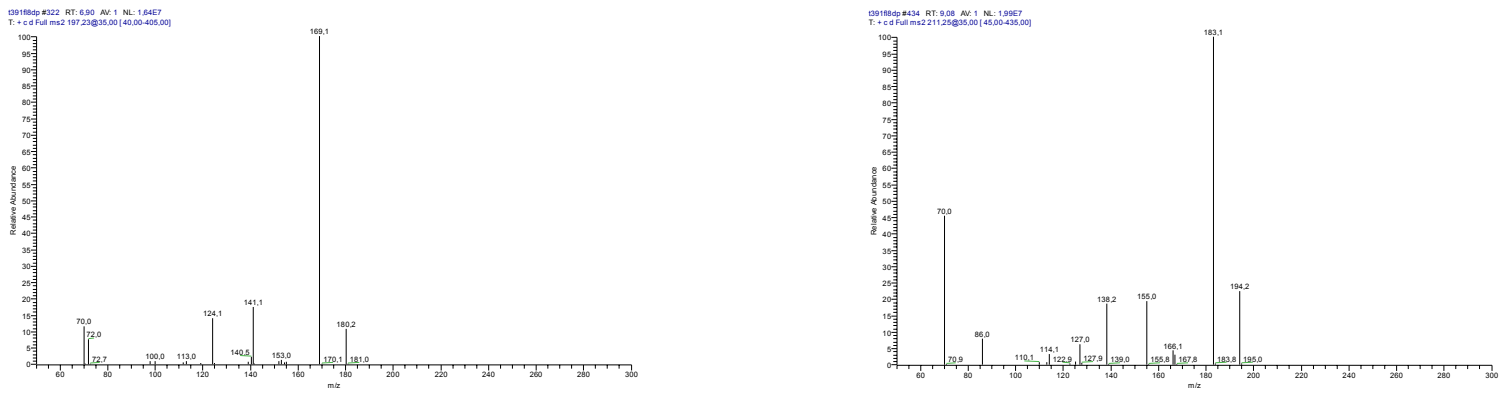

ESI-MS/MS-Spektrum von Cyclo(Val-Pro)

ESI-MS/MS-Spektrum von Cyclo(Leu-Pro)

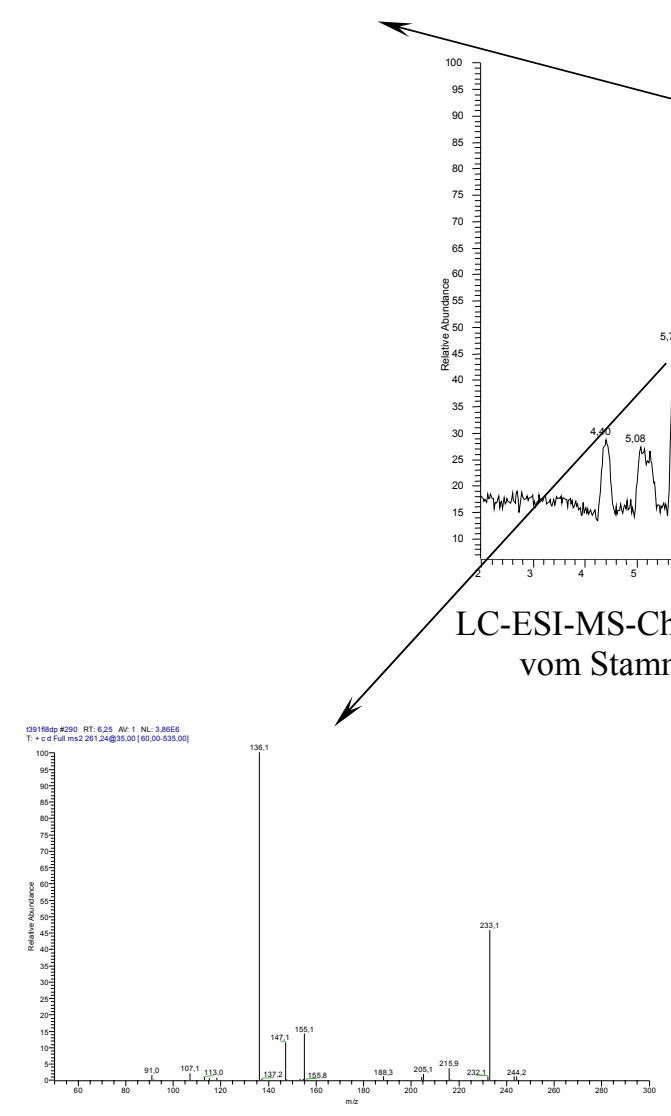

ESI-MS/MS-Spektrum von Cyclo(Tyr-Pro)
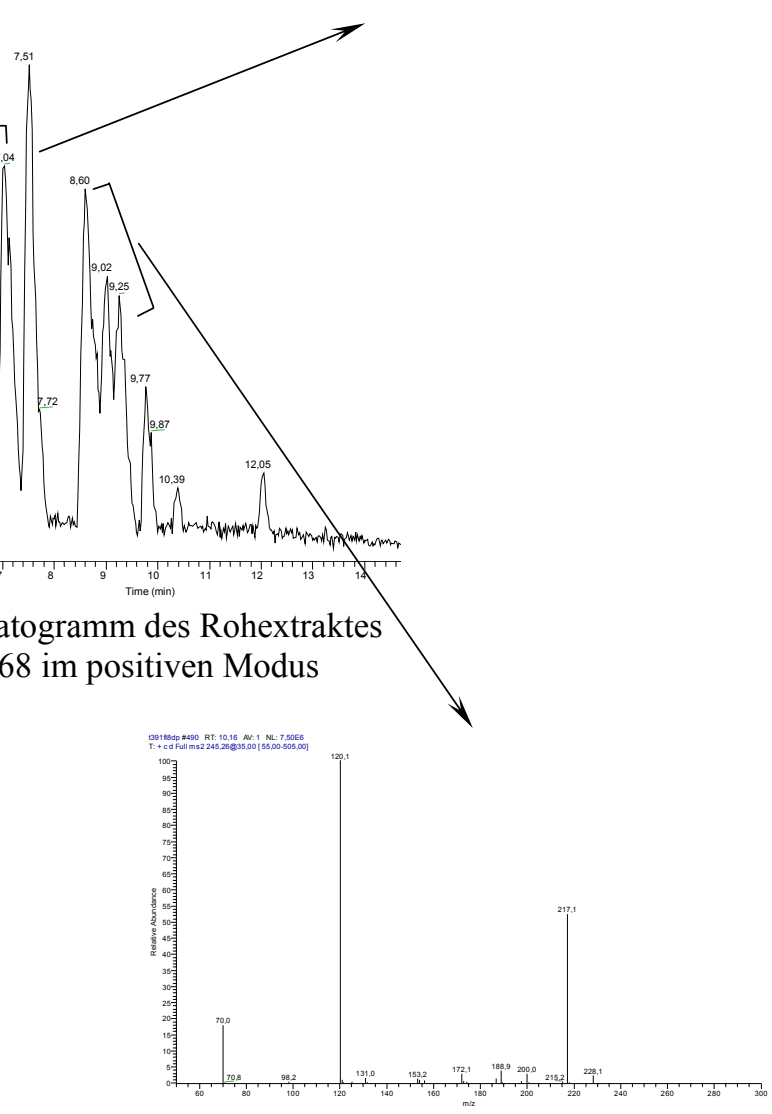

ESI-MS/MS-Spektrum von Cyclo(Phe-Pro)

Abbildung 14 Identifizierung der im Rohextrakt von Stamm T268 enthaltenen Substanzen mit Hilfe der HPLC-UV-ESI-MS/MS-Datenbank. 


\section{$5 \quad$ Fragmentierungen ausgewählter Substanzen}

Die moderne Massenspektrometrie hat sich im Bereich der Naturstoffchemie zu einem mächtigen Werkzeug entwickelt. Außer dem reinen Molekulargewicht liefert sie eine Vielzahl weiterer, wertvoller Informationen über eine Verbindung. Durch deren Kombination können bekannte Substanzen unter Nutzung von Datenbanken rasch identifiziert werden. Liegt eine noch unbekannte Verbindung vor, kann die Massenspektrometrie die Molekulargewichtsbestimmung sowie den Nachweis funktioneller Gruppen erbringen und in einigen Fällen eine komplette Strukturzuordnung ermöglichen. Letztere ist insbesondere dann möglich, wenn die Fragmentierungswege strukturanaloger Substanzen bereits im Vorfeld aufgeklärt werden konnten.

Die Sequenzanalyse von Proteinen stellt heute bereits eines der wichtigsten Anwendungsgebiete der modernen, schonenden MS-Ionisierungsverfahren in Kombination mit geeigneten MS/MS-Techniken dar. Bei der Aufklärung der Primärstruktur dieser Biopolymere handelt es sich um einen Kernbereich des als Proteomics bezeichneten Forschungsfeldes, welches die Analyse der gesamten in genomischer DNA codierten Proteine zum Ziel hat. ${ }^{32}$ Das große Interesse am Einsatz der MS-Analyse für die Peptidsequenzierung gründet sich auf der Hoffnung, durch deren Anwendung einige Nachteile des klassisch eingesetzten Edman-Abbaus ${ }^{33}$ wie den großen Arbeits- und Zeitaufwand sowie den hohen Substanzbedarf überwinden zu können.

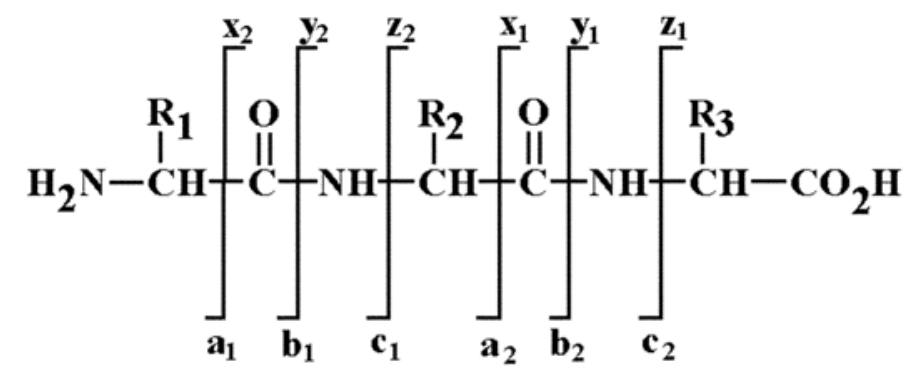

Abbildung 15 Schematische Darstellung der Fragmentierungswege eines linearen Peptides nach ROEPSTORFF. ${ }^{34}$

Bei der MS/MS-Analyse von Peptiden kommt es zur Bildung unterschiedlicher FragmentIonen (Abbildung 15), welche zum großen Teil aus der Spaltung der amidischen Bindungen resultieren. Befindet sich die Ladung am N-terminalen Fragment, spricht man von Ionen der a-, b- bzw. c-Reihe, ist sie am C-terminalen Fragment lokalisiert, bezeichnet man dieses als x-, y- bzw. z-Ion. 
Als besonders schwierig erwies sich die Aufklärung der Fragmentierungsmechanismen von Cyclopeptiden. Die hier beobachteten Fragmentierungsmuster sind durch konkurrierende Primärspaltungen verschiedener Peptidbindungen sehr komplex. Bei dieser Verbindungsklasse musste darüber hinaus auch das Problem Sequenz/Retrosequenz gelöst werden.

Das Fragmentierungsmuster eines Moleküls hängt von der verwendeten Ionisierungsmethode, der Art der Kollisionsaktivierung sowie vom eingesetzten Massenanalysator ab. ${ }^{35}$ Im Vergleich zur EI-Massenspektrometrie sind die bei der ESI-MS/MS-Massenspektrometrie erhaltenen Fragmentierungswege bisher nur in wenigen Fällen untersucht worden. ${ }^{36}$ Um deren Verständnis zu fördern, wurden einige der im Rahmen der Erstellung der HPLCUV-ESI-MS/MS-Datenbank gemessenen MS/MS-Spektren von ausgewählten Verbindungsklassen analysiert.

Die Anlagerung von $\mathrm{Na}^{+}$- bzw. $\mathrm{H}^{+}$-Ionen, welche zur Ionisierung der untersuchten Moleküle führt, erfolgt an freien Eletronenpaaren von Heteroatomen. Die geladenen Moleküle spalten nach Stoßaktivierung mit Edelgasatomen neutrale Fragmente ab. Es wird vermutet, dass die Fragmentierung in der Nähe des protonierten bzw. mit einem $\mathrm{Na}^{+}$-Ion assoziierten Atoms erfolgt. Die $\mathrm{MS}^{\mathrm{n}}$-Fragmentierung einer Verbindung mit einem Ion-Trap-Massenspektrometer kann dabei $\mathrm{zu}$ anderen Ionen führen als die MS/MS-Fragmentierung mit einem Triple-Quadrupol-Instrument. Bei ESI-Ion-Trap-Experimenten, welche auch zur Erstellung der Datenbank herangezogen wurden, kommt es ferner in manchen Fällen aufgrund von Protonwanderung zur Bildung protonierter Fragmente in der Ionenfalle.

Das zu beobachtende Fragmentierungsmuster ist auch von der Art des gebildeten MolekülAddukts abhängig. Einige Beispiele hiervon sind in der Literatur beschrieben, wie verschiedene Digitoxin-Fragmente von $[\mathrm{M}+\mathrm{H}]^{+}$- und $[\mathrm{M}+\mathrm{Na}]^{+}-$Ionen $^{37}$ sowie Unterschiede in den MS/MS-Spektren von $[\mathrm{M}+\mathrm{Na}]^{+}$- und $\left[\mathrm{M}+\mathrm{NH}_{4}\right]^{+}$-Ionen bei Oligosacchariden. ${ }^{38}$

\subsection{Actinomycine}

Bei den Actinomycinen handelt es sich um orangefarbene Sekundärmetaboliten von Streptomyceten. Ein Gemisch dieser Substanzklasse wurde 1940 von WAKSMAN als erstes aus Actinomyceten bekanntes Antibiotikum beschrieben. ${ }^{39}$ Den ersten Strukturbeweis eines Actinomycins erbrachten schließlich BROCKMANN und LACKNER 1960 mittels einer Total- 
synthese von Actinomycin $\mathrm{C}_{3}{ }^{40}$ Bis heute sind über 40 Actinomycine aus natürlichen Quellen isoliert worden.

Actinomycine interkalieren reversibel in doppelsträngige DNA, worauf ihre effiziente antitumorale Wirkung beruht. ${ }^{41}$ Dieser nicht selektive Mechanismus geht jedoch mit einer ausgeprägten Toxizität einher und führt so zu einer stark limitierten klinischen Anwendbarkeit dieser Substanzklasse. Um diesem Problem entgegenzutreten wurden zahlreiche Ansätze verfolgt, welche die Herstellung von Analoga mit verbessertem Wirkprofil zum Ziel hatten.

Chemisch bestehen Actinomycine aus einer Chromophoreinheit (3-Amino-1,8-dimethyl-2phenoxazone-4,5-dicarbonsäure), welche zwei Pentapeptidreste trägt, die ihrerseits amidisch an die beiden Carboxylgruppen gebunden sind. Jeder dieser Reste bildet einen Cyclodepsipeptidring. Bei den Aminosäuren A und V, die die Lactonringe mit dem Phenoxazinon-chromophor verbinden, handelt es sich fast ausschließlich um L-Threoninreste, deren $\beta$-Hydroxylgruppe mit der Carboxylgruppe der fünften Aminosäure in derselben Pentapeptidkette lactonisiert ist.

Bei der Strukturaufklärung von Actinomycinen spielte die Massenspektrometrie bisher eine eher untergeordnete Rolle. So wurde von einigen auf electron impact (EI), ${ }^{42}$ field desorption (FID), ${ }^{43}$ plasma desorption, ${ }^{44}$ chemical ionization $(\mathrm{CI})^{45}$ und fast atom bombardment $(\mathrm{FAB})^{46,47}$ beruhenden MS-Analysen berichtet. THOMAS und Mitarbeiter publizierten erstmals die Aufklärung der durch ES-Ionisierung (ESI) in Verbindung mit einem Tripel-Quadrupol-Detektor erhaltenen Actinomycin-Fragmente. ${ }^{48}$ Diese Experimente beruhten vor allem auf der Auswertung der von den $[\mathrm{M}+\mathrm{H}]^{+}$-Ionen gebildeten Fragmente. Die Fragmentierungswege einiger anderer Cyclodepsipeptide wurden hingegen bereits eingehend untersucht. ${ }^{49,50}$ Hier zeigte sich, dass primär Esterspaltungen bei dieser Verbindungsklasse bevorzugt ablaufen. ${ }^{51,52}$

Ein Ziel der vorliegenden Arbeit war, bereits aufgeklärte Fragmentierungswege bekannter Actinomycine zur Strukturaufklärung von neuen Vertretern dieser Substanzklasse heranzuziehen. Für die Untersuchung wurden verschiedene Actinomycine ausgewählt, die über identische wie auch unterschiedliche Pentapeptidreste verfügten. Als strukturell interessante Variationen erwiesen sich Actinomycine mit chlor- bzw. hydroxysubstituiertem Threonin sowie solche, welche über eine Esterbindung des Threoninrestes mit dem Chromophor verknüpft sind. Tabelle 2 gibt einen Überblick über die untersuchten Actinomy- 
cine, deren Fragmentierungswege ausgehend vom $[\mathrm{M}+\mathrm{Na}]^{+}$-Ion in der vorliegenden Arbeit aufgeklärt wurden.

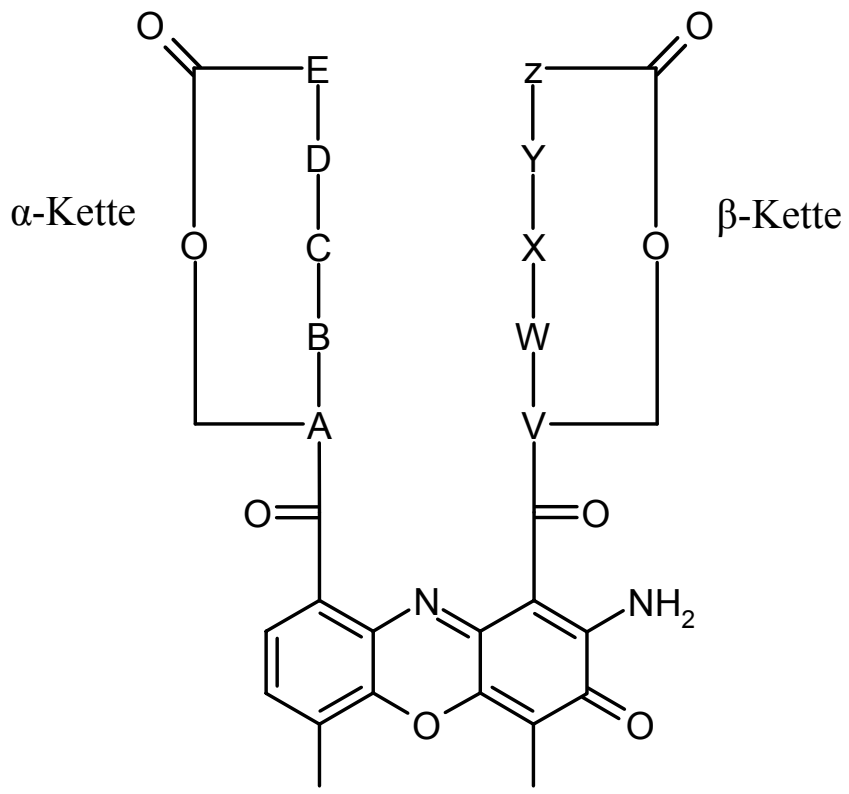

Tabelle 2 Systematische Darstellung der untersuchten Actinomycine.

\begin{tabular}{|c|c|c|c|c|c|c|c|c|c|c|}
\hline \multirow{2}{*}{$\begin{array}{c}\text { Actino- } \\
\text { mycin }\end{array}$} & \multicolumn{9}{|c|}{$\alpha$-Kette } & \multicolumn{7}{c|}{$\beta$-Kette } \\
\cline { 2 - 12 } & A & B & C & D & E & V & W & X & Y & Z \\
\hline \multicolumn{1}{|c|}{ Actinomycine mit identischen cyclischen Peptidketten } \\
\hline $\mathbf{C}_{\mathbf{3}}$ & Thr & D- $\boldsymbol{a l l e}$ & Pro & Sar & MeVal & Thr & D- $\boldsymbol{a l l e}$ & Pro & Sar & MeVal \\
\hline D & Thr & D-Val & Pro & Sar & MeVal & Thr & D-Val & Pro & Sar & MeVal \\
\hline $\mathbf{F}_{\mathbf{3}}$ & Thr & D- $\boldsymbol{a I l e}$ & Sar & Sar & MeVal & Thr & D- $\boldsymbol{a l l e}$ & Sar & Sar & MeVal \\
\hline
\end{tabular}

Actinomycine mit unterschiedlichen cyclischen Peptidketten

\begin{tabular}{|c|c|c|c|c|c|c|c|c|c|c|}
\hline $\mathbf{C}_{2}$ & Thr & D-Val & Pro & Sar & MeVal & Thr & D-aIle & Pro & Sar & MeVal \\
$\mathbf{X}_{2}$ & Thr & D-Val & Pro & Sar & MeVal & Thr & D-Val & OPro & Sar & MeVal \\
$\mathbf{X}_{\mathbf{0 \beta}}$ & Thr & D-Val & Pro & Sar & MeVal & Thr & D-Val & Hyp & Sar & MeVal \\
\hline
\end{tabular}

Actinomycine mit unterschiedlichen cyclischen Peptidketten und substituiertem Threonylrest

\begin{tabular}{|c|c|c|c|c|c|c|c|c|c|c|}
\hline $\mathbf{Z}_{\mathbf{1}}$ & Thr & D-Val & HMPro & Sar & MeVal & HThr & D-Val & MOPro & Sar & MeAla \\
$\mathbf{Z}_{\mathbf{7}}$ & Thr & D-Val & HMPro & Sar & MeVal & CIThr & D-Val & Hyp & Sar & MeAla \\
$\mathbf{G}_{\mathbf{2}}$ & Thr & D-Val & HMPro & Sar & MeVal & CIThr & D-Val & Pro & Sar & MeAla \\
$\mathbf{G}_{3}$ & Thr & D-Val & HMPro & Sar & MeVal & HThr & D-Val & Pro & Sar & MeAla \\
\hline
\end{tabular}

Actinomycine mit unterschiedlichen cyclischen Peptidketten und außergewöhnlicher Verknüpfung des Threonylrestes

\begin{tabular}{|l|c|c|c|c|c|c|c|c|c|c|}
$\mathbf{G}_{\mathbf{x}}$ & Thr & D-Val & HMPro & Sar & MeVal & HThr $^{*}$ & D-Val & Pro & Sar & MeAla \\
\hline
\end{tabular}


Unterschiede zu Actinomycin D sind fett gedruckt $(\mathrm{MeVal}=N$-Methylvalin, alle $=$ alloIsoleucin, MeAla = N-Methylalanin, HMPro = trans-3-Hydroxy-cis-5-methylprolin, OPro = 3-Oxoprolin, MOPro = cis-5-Methyl-3-oxoprolin, Hyp = trans-4-Hydroxyprolin, HThr = 4-Hydroxy-threonin, ClThr = 4-Chlorthreonin, *Verknüpfung von Threonin zwischen dem Chromophor und Peptidkette ist umgekehrt).

Abbildung 16 zeigt das MS/MS-Spektrum des $[\mathrm{M}+\mathrm{Na}]^{+}$-Ions von Actinomycin D ( $m / z$ 1277). Eine schematische Darstellung der zugrundeliegenden Fragmentierungswege ist in Abbildung 17 wiedergegeben.

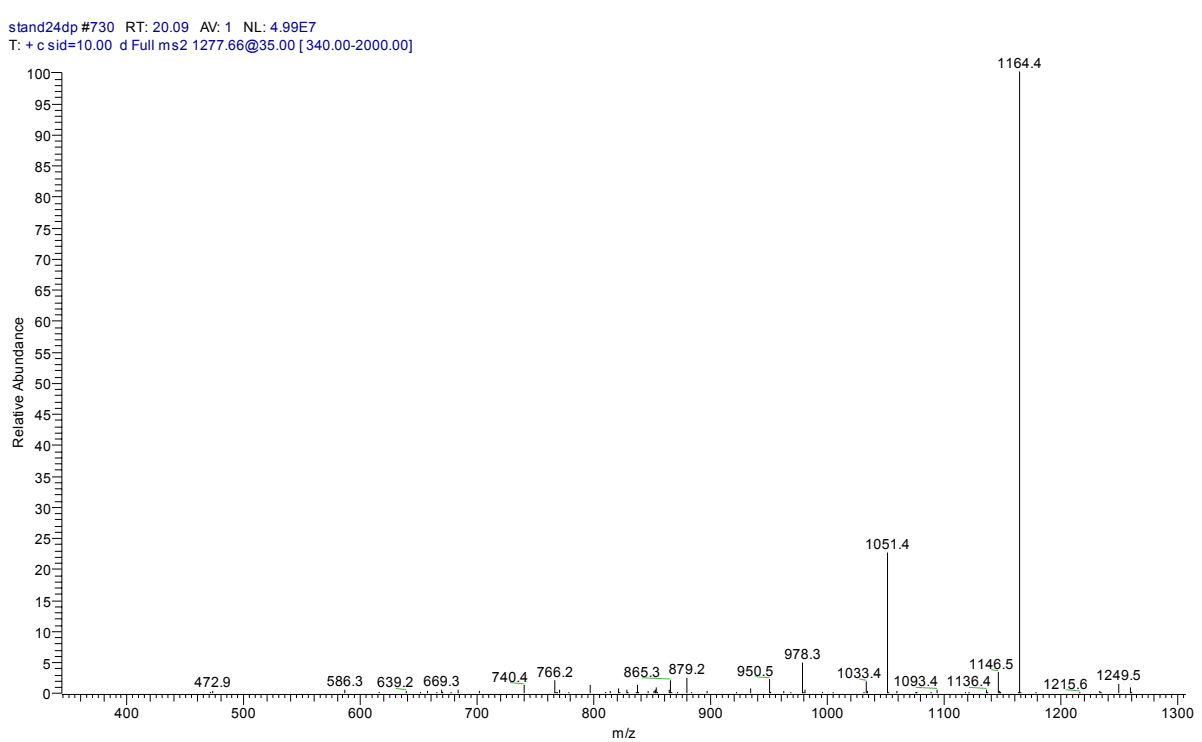

Abbildung 16 MS/MS-Spektrum des $[\mathrm{M}+\mathrm{Na}]^{+}$-Ions von Actinomycin D.

Gemäß bereits bekannten Mechanismen findet hier zunächst vermutlich eine Öffnung der beiden Peptidringe per Esterspaltung statt. Im nächsten Schritt werden nacheinander die in Position 5 befindlichen Aminosäuren abgespalten. Es folgt die schrittweise Freisetzung der Aminosäuren einer Kette, wobei gelegentlich Kohlenmonoxid- sowie Wassermoleküle eliminiert werden. Da $\alpha$ - und $\beta$-Kette dieser Substanz identisch sind, sind auch die gebildeten Fragmente gleich, was einerseits das MS/MS-Spektrum vereinfacht, eine Unterscheidung der beiden Cyclopeptidylreste jedoch verhindert. 

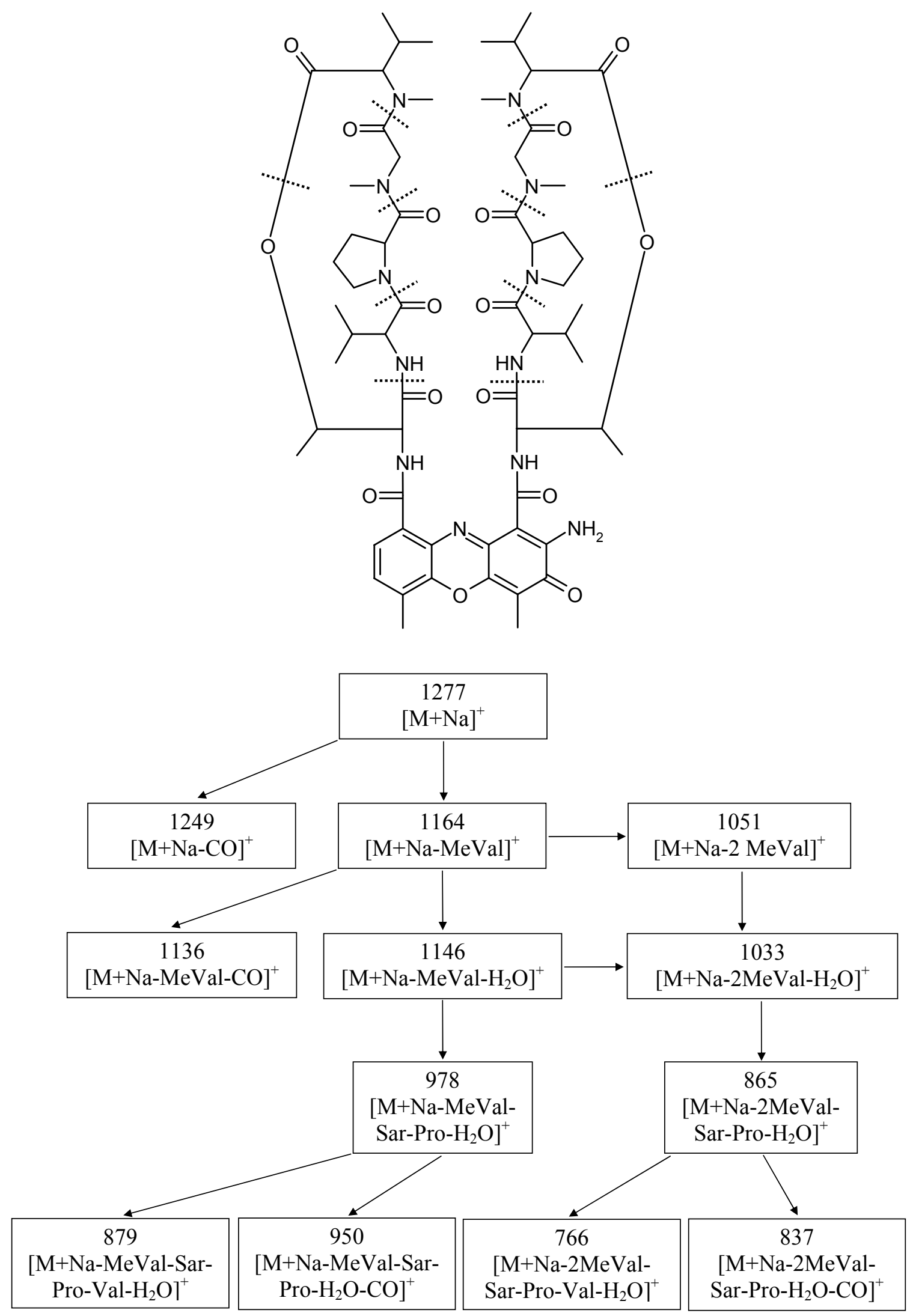

Abbildung 17 Schematische Darstellung der Fragmentierungswege von Actinomycin D. 
Tabelle 3 Beobachtete Fragmentbildung für $[\mathrm{M}+\mathrm{Na}]^{+}$-Ionen von Actinomycinen mit identischen cyclischen Peptidketten.

\begin{tabular}{|c|c|c|c|}
\hline \multirow{2}{*}{ Fragmention } & \multicolumn{3}{|c|}{ Fragmentmassen von Actinomycin $[\mathrm{m} / z]$} \\
\cline { 2 - 4 } & $\mathrm{C}_{3}$ & $\mathrm{D}$ & $\mathrm{F}_{3}$ \\
\hline$[\mathrm{M}+\mathrm{Na}]^{+}$ & 1305 & 1277 & 1253 \\
\hline$[\mathrm{M}+\mathrm{Na}-\mathrm{CO}]^{+}$ & 1277 & 1249 & 1225 \\
\hline$[\mathrm{M}+\mathrm{Na}-\mathrm{E}]^{+}$ & 1192 & 1164 & 1140 \\
\hline$\left[\mathrm{M}+\mathrm{Na}-\mathrm{E}-\mathrm{H}_{2} \mathrm{O}\right]^{+}$ & 1174 & 1146 & 1122 \\
\hline$[\mathrm{M}+\mathrm{Na}-\mathrm{E}-\mathrm{CO}]^{+}$ & - & 1136 & - \\
\hline$[\mathrm{M}+\mathrm{Na}-\mathrm{E}-\mathrm{Z}]^{+}$ & 1079 & 1051 & 1027 \\
\hline$\left[\mathrm{M}+\mathrm{Na}-\mathrm{E}-\mathrm{Z}-\mathrm{H}_{2} \mathrm{O}\right]^{+}$ & 1061 & 1033 & 1009 \\
\hline$\left[\mathrm{M}+\mathrm{Na}-\mathrm{E}-\mathrm{D}-\mathrm{H}_{2} \mathrm{O}\right]^{+}$ & - & - & 1051 \\
\hline$\left[\mathrm{M}+\mathrm{Na}-\mathrm{E}-\mathrm{D}-\mathrm{C}-\mathrm{H}_{2} \mathrm{O}\right]^{+}$ & 1006 & 978 & 980 \\
\hline$\left[\mathrm{M}+\mathrm{Na}-\mathrm{E}-\mathrm{D}-\mathrm{C}-\mathrm{H}_{2} \mathrm{O}-\mathrm{CO}\right]^{+}$ & 978 & 950 & - \\
\hline$\left[\mathrm{M}+\mathrm{Na}-\mathrm{E}-\mathrm{D}-\mathrm{C}-\mathrm{B}-\mathrm{H}_{2} \mathrm{O}\right]^{+}$ & 893 & 879 & 867 \\
\hline$\left[\mathrm{M}+\mathrm{Na}-\mathrm{E}-\mathrm{D}-\mathrm{C}-\mathrm{B}-\mathrm{A}-\mathrm{H}_{2} \mathrm{O}\right]^{+}$ & - & - & 784 \\
\hline$\left[\mathrm{M}+\mathrm{Na}-\mathrm{E}-\mathrm{Z}-\mathrm{D}-\mathrm{C}-\mathrm{H}_{2} \mathrm{O}\right]^{+}$ & 893 & 865 & 867 \\
\hline$\left[\mathrm{M}+\mathrm{Na}-\mathrm{E}-\mathrm{Z}-\mathrm{D}-\mathrm{C}-\mathrm{H}_{2} \mathrm{O}-\mathrm{CO}\right]^{+}$ & 865 & 837 & - \\
\hline$\left[\mathrm{M}+\mathrm{Na}-\mathrm{E}-\mathrm{Z}-\mathrm{D}-\mathrm{C}-\mathrm{B}-\mathrm{H}_{2} \mathrm{O}\right]^{+}$ & 780 & 766 & 754 \\
\hline
\end{tabular}

Eine Variation der dritten Aminosäure in einer der Peptidketten, wie im Fall von Actinomycin $\mathrm{X}_{0 ß}$, führt nach einer bestimmten Anzahl von Fragmentierungsschritten zur Bildung unterschiedlicher Bruchstücke für beide Cyclen. Dies setzt jedoch eine Fragmentierung mindestens bis zur dritten Position voraus. Abbildung 18 zeigt eine schematische Darstellung der Fragmentierungswege des $\left[\mathrm{M}+\mathrm{Na}^{+}\right]$-Ions $(\mathrm{m} / \mathrm{z} 1293)$ von Actinomycin $\mathrm{X}_{0 \beta}$. Eine Übersicht über die Fragmentionen einiger unsymmetrischer Actinomycine gibt Tabelle 4. 

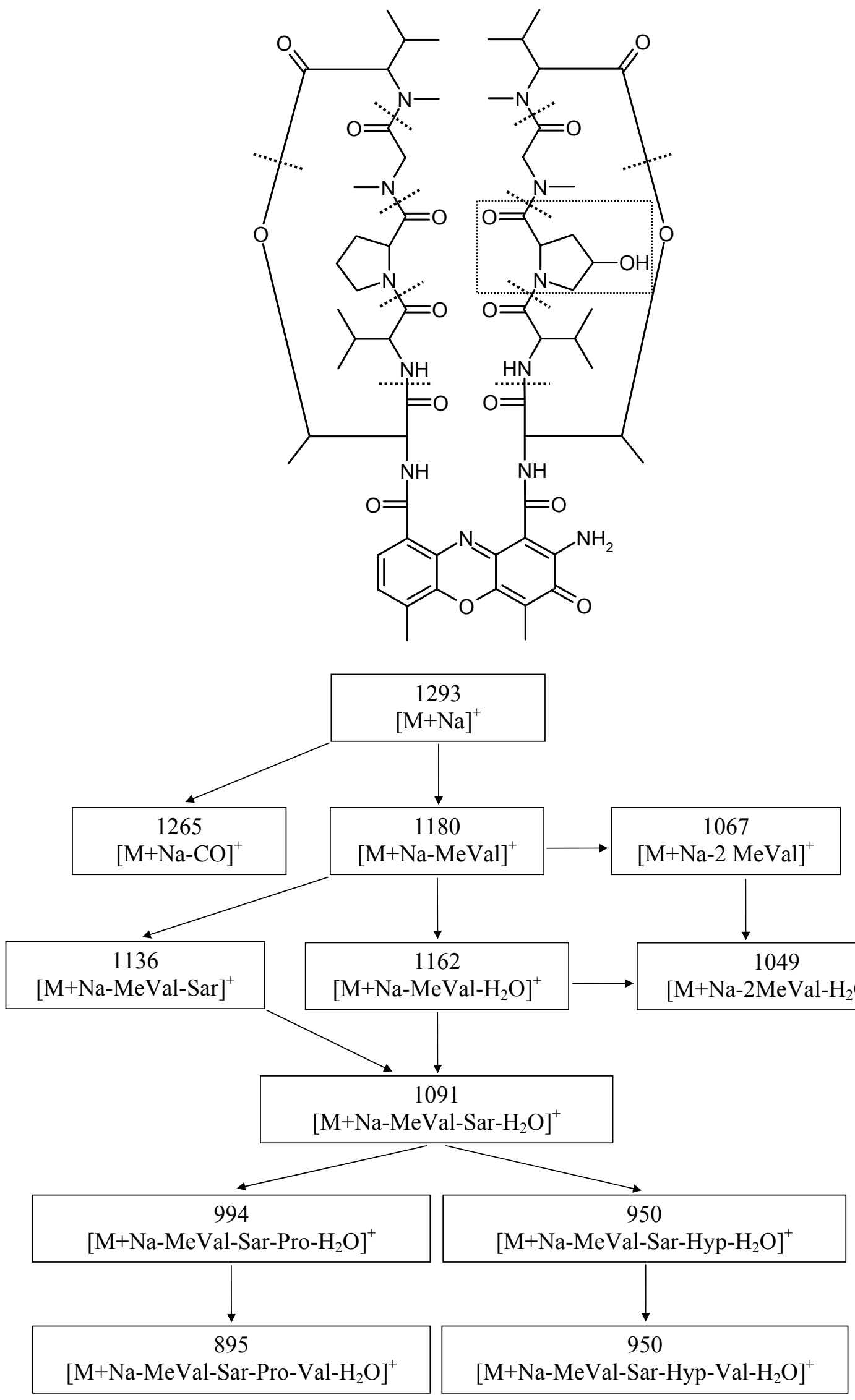

Abbildung 18 Schematische Darstellung der Fragmentierungswege von Actinomycin $\mathrm{X}_{0 \beta}$. 
Tabelle 4 Beobachtete Fragmentbildung der $[\mathrm{M}+\mathrm{Na}]^{+}$-Ionen von Actinomycinen mit unterschiedlichen cyclischen Peptidketten.

\begin{tabular}{|c|c|c|c|}
\hline \multirow{2}{*}{ Fragmention } & \multicolumn{3}{|c|}{ Fragmentmassen von Actinomycin $[\mathrm{m} / z]$} \\
\cline { 2 - 4 } & $\mathrm{C}_{2}$ & $\mathrm{X}_{2}$ & $\mathrm{X}_{0 \beta}$ \\
\hline$[\mathrm{M}+\mathrm{Na}]^{+}$ & 1291 & 1291 & 1293 \\
\hline$[\mathrm{M}+\mathrm{Na}-\mathrm{CO}]^{+}$ & 1263 & 1263 & 1265 \\
\hline$[\mathrm{M}+\mathrm{Na}-\mathrm{E}]^{+}$ & 1178 & 1178 & 1180 \\
\hline$\left[\mathrm{M}+\mathrm{Na}-\mathrm{E}-\mathrm{H}_{2} \mathrm{O}\right]^{+}$ & 1160 & 1160 & 1162 \\
\hline$[\mathrm{M}+\mathrm{Na}-\mathrm{E}-\mathrm{D}]^{+}$ & - & 1107 & 1109 \\
\hline$\left[\mathrm{M}+\mathrm{Na}-\mathrm{E}-\mathrm{D}-\mathrm{H}_{2} \mathrm{O}\right]^{+}$ & 1089 & - & 1091 \\
\hline$[\mathrm{M}+\mathrm{Na}-\mathrm{E}-\mathrm{Z}]^{+}$ & 1065 & 1065 & 1067 \\
\hline$\left[\mathrm{M}+\mathrm{Na}-\mathrm{E}-\mathrm{Z}-\mathrm{H}_{2} \mathrm{O}\right]^{+}$ & 1047 & - & 1049 \\
\hline$\left[\mathrm{M}+\mathrm{Na}-\mathrm{E}-\mathrm{D}-\mathrm{C}-\mathrm{H}_{2} \mathrm{O}\right]^{+}$ & 992 & 992 & 994 \\
\hline$\left[\mathrm{M}+\mathrm{Na}-\mathrm{Z}-\mathrm{Y}-\mathrm{X}-\mathrm{H}_{2} \mathrm{O}\right]^{+}$ & 992 & 978 & 978 \\
\hline$\left[\mathrm{M}+\mathrm{Na}-\mathrm{E}-\mathrm{D}-\mathrm{C}-\mathrm{H}_{2} \mathrm{O}-\mathrm{CO}\right]^{+}$ & 964 & 964 & 966 \\
\hline$\left[\mathrm{M}+\mathrm{Na}-\mathrm{E}-\mathrm{D}-\mathrm{C}-\mathrm{B}-\mathrm{H}_{2} \mathrm{O}\right]^{+}$ & 893 & 893 & 895 \\
\hline$\left[\mathrm{M}+\mathrm{Na}-\mathrm{Z}-\mathrm{Y}-\mathrm{X}-\mathrm{W}-\mathrm{H}_{2} \mathrm{O}\right]^{+}$ & 879 & 879 & 879 \\
\hline$\left[\mathrm{M}+\mathrm{Na}-\mathrm{Z}-\mathrm{Y}-\mathrm{X}-\mathrm{W}-\mathrm{H}_{2} \mathrm{O}-\mathrm{CO}\right]^{+}$ & 851 & 851 & - \\
\hline
\end{tabular}

Bei den Actinomycinen der Z- und G-Reihe, welche unterschiedliche Aminosäurereste in Position 2 aufweisen, sind bereits bei den ersten Fragmentierungsschritten unterschiedliche Fragmentmassen für beide Peptidketten erkennbar. Trotz der Möglichkeit einer Sequenzzuordnung der einzelnen Aminosäure-Ketten können diese mittels der eingesetzten Methode nicht eindeutig einer Position des Chromophorgerüsts zugeteilt werden.

Liegt ein Threoninrest chlorsubstituiert vor, ergibt sich das Fragment höchster Intensität aus einer Abspaltung von Chlorwasserstoff. Ob dieses Fragment vor oder nach Öffnung der Peptidringe gebildet wird, ist nicht bekannt. Befindet sich hingegen ein hydroxysubstituierter Threoninrest im Molekül, resultieren die Fragmentionen höchster Intensität aus einer Abspaltung der Aminosäuren in Position 5. Nach sukzessiver Spaltung aller Amidbindungen der Peptidketten kommt es zur Bildung eines Acetaldehyd-Fragments der Masse 44. Dieses entsteht vermutlich über eine $\alpha$-Spaltung des ursprünglichen Threoninrests. 

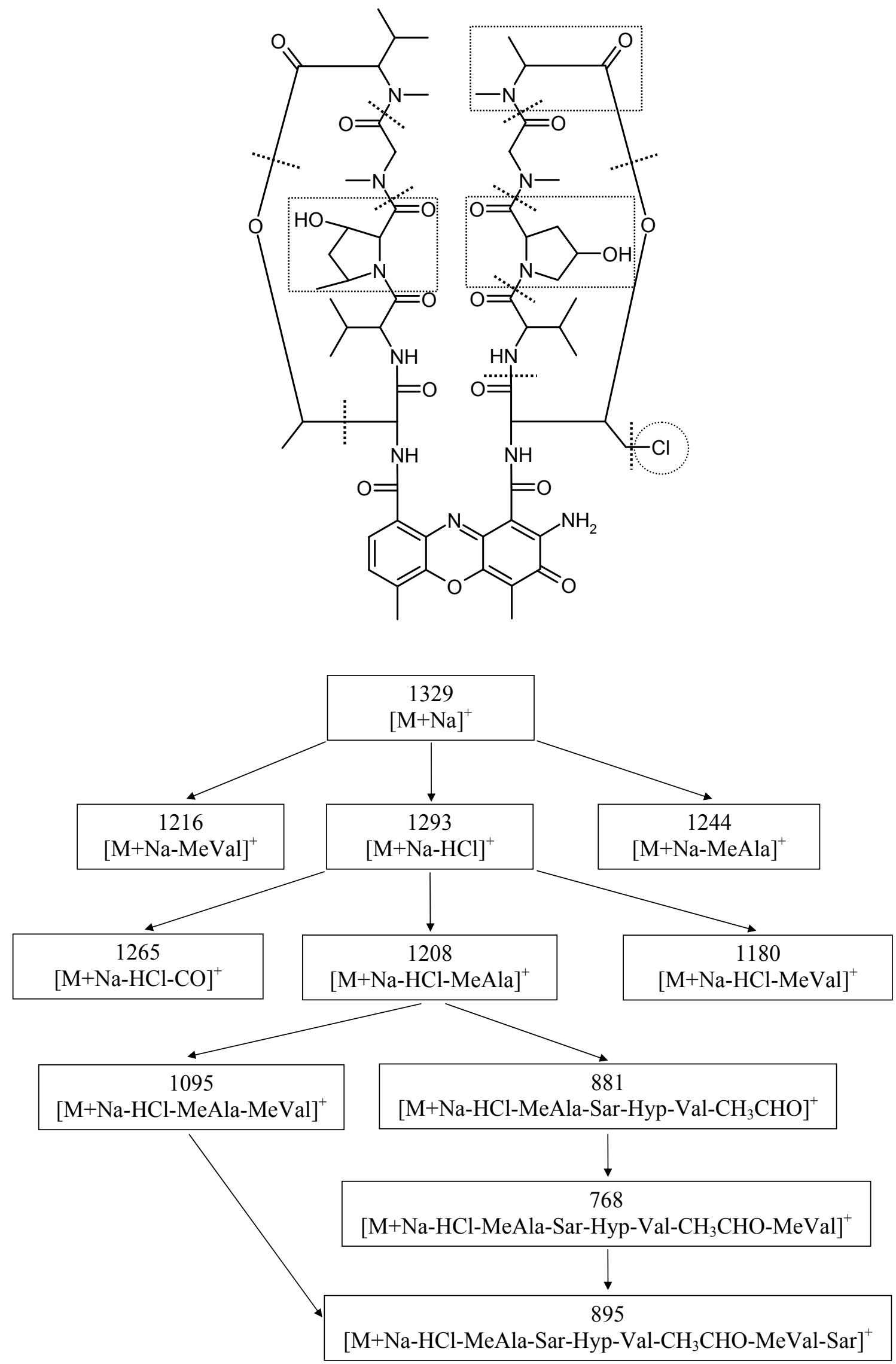

Abbildung 19 Schematische Darstellung der Fragmentierungswege von Actinomycin $Z_{7}$. 
Tabelle 5 Beobachtete Fragmentierung von $[\mathrm{M}+\mathrm{Na}]^{+}$-Ionen unsymmetrischer Actinomycine mit unterschiedlichen Aminosäuren in der 5. Position sowie Hydroxybzw. Chloridsubstitution (Q) an einem der Threoninreste.

\begin{tabular}{|c|c|c|c|c|}
\hline \multirow{2}{*}{ Fragmention } & \multicolumn{4}{|c|}{ Fragmentmassen von Actinomycin $[\mathrm{m} / \mathrm{z}]$} \\
\hline & $\mathrm{Z}_{1}$ & $\mathrm{Z}_{7}$ & $\mathrm{G}_{2}$ & $\mathrm{G}_{3}$ \\
\hline$[\mathrm{M}+\mathrm{Na}]^{+}$ & 1323 & 1329 & 1313 & 1295 \\
\hline$[\mathrm{M}+\mathrm{Na}-\mathrm{Q}]^{+}$ & 1305 & 1293 & 1277 & 1277 \\
\hline$[\mathrm{M}+\mathrm{Na}-\mathrm{CO}]^{+}$ & 1295 & - & - & 1267 \\
\hline$[\mathrm{M}+\mathrm{Na}-\mathrm{Q}-\mathrm{CO}]^{+}$ & - & 1265 & 1249 & - \\
\hline$[\mathrm{M}+\mathrm{Na}-\mathrm{E}]^{+}$ & 1238 & 1216 & 1200 & 1182 \\
\hline$[\mathrm{M}+\mathrm{Na}-\mathrm{Z}]^{+}$ & 1210 & 1244 & 1228 & 1210 \\
\hline$[\mathrm{M}+\mathrm{Na}-\mathrm{E}-\mathrm{Q}]^{+}$ & 1192 & 1180 & 1164 & 1164 \\
\hline$[\mathrm{M}+\mathrm{Na}-\mathrm{Z}-\mathrm{Q}]^{+}$ & 1220 & 1208 & 1192 & 1192 \\
\hline$[\mathrm{M}+\mathrm{Na}-\mathrm{E}-\mathrm{Z}]^{+}$ & 1125 & - & - & 1097 \\
\hline$[\mathrm{M}+\mathrm{Na}-\mathrm{E}-\mathrm{Z}-\mathrm{Q}]^{+}$ & 1107 & 1095 & 1079 & 1079 \\
\hline$[\mathrm{M}+\mathrm{Na}-\mathrm{E}-\mathrm{D}]^{+}$ & - & - & - & 1111 \\
\hline$[\mathrm{M}+\mathrm{Na}-\mathrm{E}-\mathrm{Z}-\mathrm{D}]^{+}$ & 1054 & - & - & - \\
\hline$[\mathrm{M}+\mathrm{Na}-\mathrm{E}-\mathrm{D}-\mathrm{C}-\mathrm{Q}]^{+}$ & 994 & - & - & 966 \\
\hline$[\mathrm{M}+\mathrm{Na}-\mathrm{Z}-\mathrm{Y}-\mathrm{X}-\mathrm{Q}]^{+}$ & 1024 & - & - & - \\
\hline$[\mathrm{M}+\mathrm{Na}-\mathrm{E}-\mathrm{D}-\mathrm{C}-\mathrm{Q}-\mathrm{CO}]^{+}$ & 966 & - & - & 938 \\
\hline$[\mathrm{M}+\mathrm{Na}-\mathrm{E}-\mathrm{D}-\mathrm{C}-\mathrm{B}-\mathrm{Q}]^{+}$ & 895 & - & - & - \\
\hline$[\mathrm{M}+\mathrm{Na}-\mathrm{Z}-\mathrm{Y}-\mathrm{X}-\mathrm{W}-\mathrm{Q}]^{+}$ & 925 & - & - & - \\
\hline$\left[\mathrm{M}+\mathrm{Na}-\mathrm{Z}-\mathrm{Y}-\mathrm{X}-\mathrm{W}-\mathrm{Q}-\mathrm{CH}_{3} \mathrm{CHO}\right]^{+}$ & 881 & 881 & 881 & 881 \\
\hline$\left[\mathrm{M}+\mathrm{Na}-\mathrm{E}-\mathrm{Z}-\mathrm{Y}-\mathrm{X}-\mathrm{W}-\mathrm{Q}-\mathrm{CH}_{3} \mathrm{CHO}\right]^{+}$ & 768 & 768 & 768 & 768 \\
\hline$\left[\mathrm{M}+\mathrm{Na}-\mathrm{E}-\mathrm{D}-\mathrm{Z}-\mathrm{Y}-\mathrm{X}-\mathrm{W}-\mathrm{Q}-\mathrm{CH}_{3} \mathrm{CHO}\right]^{+}$ & 697 & 697 & 697 & 697 \\
\hline
\end{tabular}

Tabelle 5 zeigt eine Liste der detektierten Fragmentionen einiger Actinomycine der Reihen $\mathrm{Z}$ und G. Hierbei ist auffällig, dass Actinomycine, welche einen chlorsubstituierten Threoninrest beinhalten (Actinomycin $\mathrm{Z}_{7}$ und $\mathrm{G}_{2}$ ), eine geringere $\mathrm{Zahl}$ an Fragmenten bildet als solche, welche über eine hydroxysubstituierte Threoningruppierung verfügen (Actinomy$\operatorname{cin} Z_{1}$ und $G_{3}$ ). Je mehr Fragmente eine Substanz nach Beschuss mit einer bestimmten Kollisionsenergie liefert, desto mehr Informationen können aus den entsprechenden Spektren erhalten werden. In Fällen von Actinomycinen mit hydroxylierten Threoninresten konnte die schrittweise Zuordnung der einzelnen Aminosäuren erfolgen, während es bei 
Anwesenheit chlorierter Threoningruppen zu einer unspezifischen Abspaltung mehrerer Aminosäuren gleichzeitig kam.

Kürzlich konnten die neuartigen Actinomycine $\mathrm{Z}_{7}, \mathrm{G}_{2}$ und $\mathrm{G}_{3}$ aus Streptomyces sp. CS210 isoliert werden. ${ }^{*}$ Zusätzlich wurde aus diesem Stamm ein Actinomycin mit ungewöhnlicher Verknüpfung am Threoninrest gefunden. Bei der Strukturaufklärung ergab sich, dass hier eine cyclische Peptidkette über eine Esterbindung mit dem Chromophorgerüst verknüpft ist. Dies hat deutliche Konsequenzen für die mit ESI-MS/MS erhaltenen Fragmente. Es zeigte sich, dass die Aminosäurereste der betreffenden Peptidkette nicht nacheinander abgespalten werden, sondern es vielmehr zur Freisetzung der gesamten Kette kommt. Die Fragmentierung der zweiten Peptidkette verläuft nach dem üblichen Muster, was deren Sequenzzuordnung erlaubt. Abbildung 20 zeigt eine schematische Darstellung der Fragmentierungswege von Actinomycin $\mathrm{G}_{6}$.

Die Interpretation der Fragmentierung des Natriumadduktes bereits bekannter Actinomycine lieferte wertvolle Informationen, die in den meisten Fällen zu einer eindeutigen Identifizierung der vorliegenden Verbindung ausreichten. Handelt es sich um noch unbekannte Actinomycine, ermöglicht die beschriebene, zeitsparende Methode vielfach die Zuordnung der kompletten Aminosäuresequenz. Als schwierig erwiesen sich jedoch die Bestimmung des Substitutionsortes der Peptidketten am Chromophorgerüst sowie die Unterscheidung von Aminosäureisomeren. Hier musste auf die Hilfe von NMR-spektroskopischen Daten zurückgegriffen werden.

\footnotetext{
* J. Bitzer und Prof. Dr. A. Zeeck danke ich für die Bereitstellung der Actinomycine.
} 

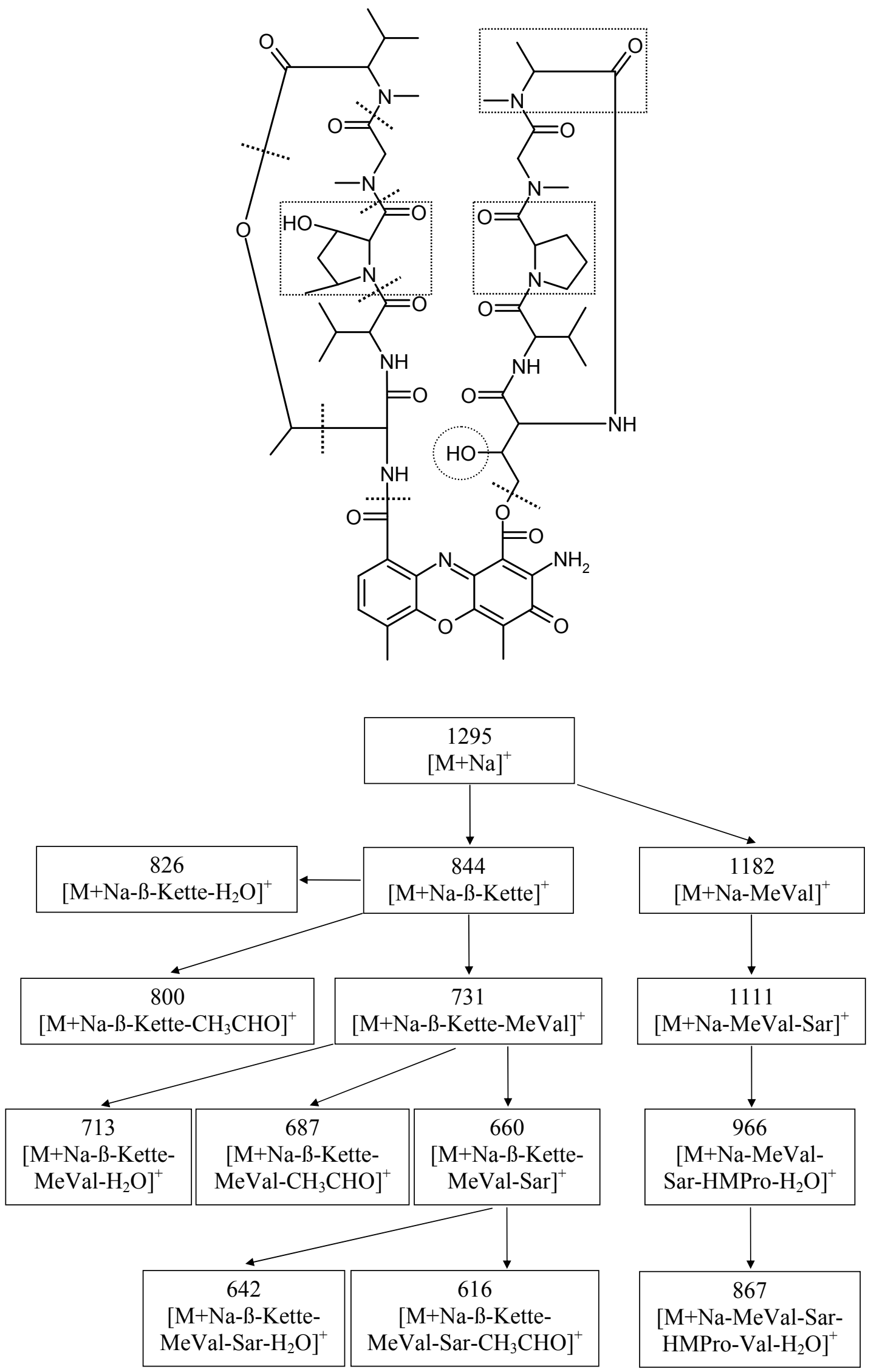

Abbildung 20 Schematische Darstellung der Fragmentierungswege von Actinomycin $\mathrm{G}_{6}$. 


\subsection{Diketopiperazine}

Eine aus Bakterienkulturen häufig isolierte Verbindungsklasse ist die der cyclischen Dipeptide, welche unter dem Name Diketopiperazine bekannt sind. Diese Substanzen beinhalten häufig nichtribosomal synthetisierte Aminosäuren, was die Bildung isomerer Strukturen ermöglicht. Die meisten in den Rohextrakten gefundenen Diketopiperazine weisen eine Prolylgruppe auf, wobei am häufigsten die Verbindungen Cyclo(prolylvalyl) (15), Cyclo(leucylprolyl) (16), Cyclo(phenylalanylprolyl) (17) und Cyclo(prolyltyrosyl) (18) gefunden werden.<smiles>CC(C)C1NC(=O)C2CCCN2C1=O</smiles>

15<smiles>O=C1NC(Cc2ccccc2)C(=O)N2CCCC12</smiles>

17<smiles>CC(C)CC1NC(=O)C2CCCN2C1=O</smiles>

16<smiles>O=C1NC(Cc2ccc(O)cc2)C(=O)N2CCCC12</smiles>

18

Aufgrund der guten Ionisierbarkeit von Peptiden und der vergleichsweise hohen gebildeten Substanzmengen waren die entsprechenden Peaks im Totalionenstrom-Chromatogramm der Rohextrakte sehr auffällig. Anhand des Fragmentierungsmusters und der Retentionszeiten konnten die gefundenen Diketopiperazine durch eine Suche in der HPLC-UV-ESIMS/MS-Datenbank schnell identifiziert werden. Die Methode stößt jedoch an ihre Grenze, wenn es um die Unterscheidung von Isomeren geht, welche verschiedene Konfiguration (cis- und trans-Form) bzw. unterschiedliche Konstitution (z. B. Leucin und Isoleucin) aufweisen. Aufgrund der identischen Fragmentierungsmuster sowie der sehr ähnlichen Retentionszeiten können solche Verbindungen per HPLC-MS/MS nicht eindeutig identifiziert werden. 
Um die Fragmentierungswege von mittels ESI ionisierten Peptiden besser verstehen zu können, wurde im Rahmen dieser Arbeit versucht, die in den MS/MS-Spektren auftretenden Diketopiperazin-Fragmente aufzuklären.<smiles>CC(C)C1NC(=O)C2CCCN2C1=O</smiles>

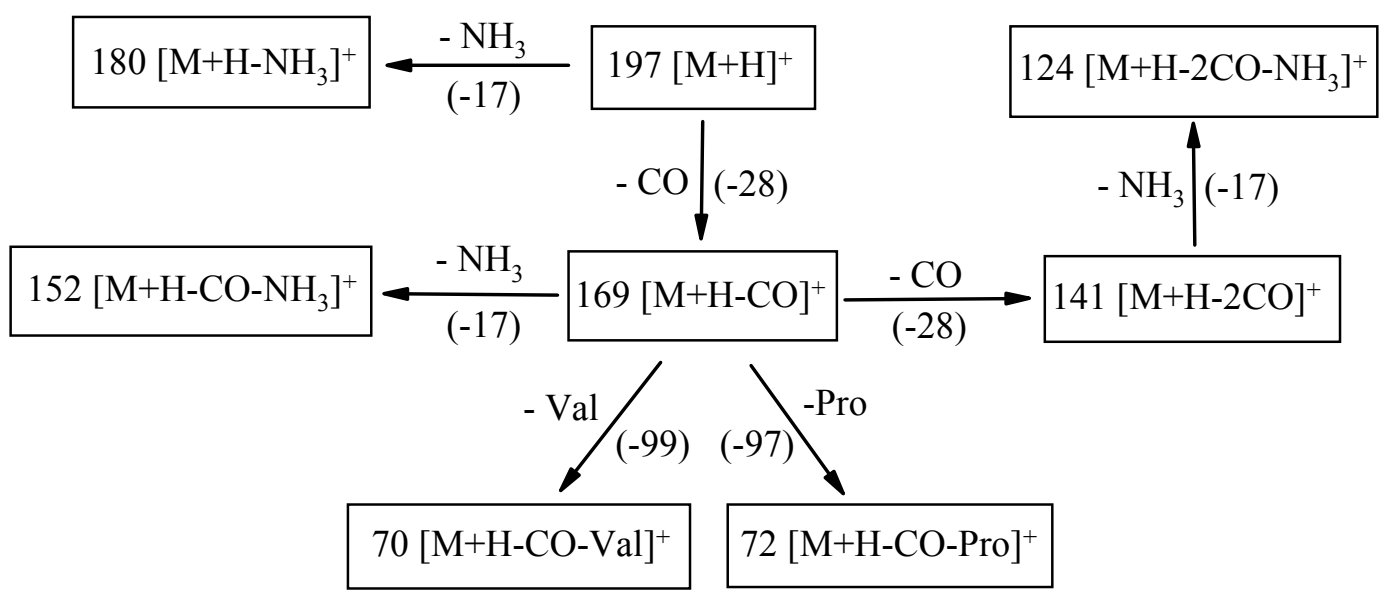

Abbildung 21 Vorschlag eines Fragmentierungsschemas für Cyclo(prolylvalyl) (15).

In Abbildung 21 ist das Fragmentierungsschema eines Diketopiperazins dargestellt. Die Zusammensetzung aller gefundenen Fragmente wurde per Hochauflösung verifiziert. Ammoniak- und Kohlenstoffmonoxidabspaltung konnten hier häufig beobachtet werden. Zusätzlich konnte die Bildung von Immoniumionen der beiden Aminosäuren nachgewiesen werden. Aus diesem Grund wurde vermutet, dass die Wahrscheinlichkeit einer Protonierung des Moleküls an den beiden Aminosäureresten gleich ist. Der genaue Fragmentierungsmechanismus dieser Substanzklasse ist bisher jedoch nicht vollständig aufgeklärt.

Tabelle 6 zeigt eine Liste der Fragmentionen, die von den vier am häufigsten vorkommenden Diketopiperazinen gebildet werden. 
Tabelle 6 Fragmente von häufig isolierten Diketopiperazinen. (X: Aminosäure außer Prolin)

\begin{tabular}{|c|c|c|c|c|}
\hline Fragmention & $\begin{array}{c}\text { Cyclo(Pro- } \\
\text { Val) (15) }\end{array}$ & $\begin{array}{c}\text { Cyclo(Leu- } \\
\text { Pro) (16) }\end{array}$ & $\begin{array}{c}\text { Cyclo(Phenyl- } \\
\text { ala-Pro) (17) }\end{array}$ & $\begin{array}{c}\text { Cyclo(Pro- } \\
\text { Tyr) (18) }\end{array}$ \\
\hline$[\mathrm{M}+\mathrm{H}]^{+}$ & 197 & 211 & 245 & 261 \\
\hline$\left[\mathrm{M}+\mathrm{H}-\mathrm{NH}_{3}\right]^{+}$ & 180 & 194 & 228 & 244 \\
\hline$[\mathrm{M}+\mathrm{H}-\mathrm{CO}]^{+}$ & 169 & 183 & 217 & 233 \\
\hline$[\mathrm{M}+\mathrm{H}-2 \mathrm{CO}]^{+}$ & 141 & 155 & 189 & 205 \\
\hline$\left[\mathrm{M}+\mathrm{H}-\mathrm{CO}-\mathrm{NH}_{3}\right]^{+}$ & 152 & 166 & 200 & 216 \\
\hline$\left[\mathrm{M}+\mathrm{H}-2 \mathrm{CO}-\mathrm{NH}_{3}\right]^{+}$ & 124 & 138 & 172 & 188 \\
\hline$[\mathrm{M}+\mathrm{H}-\mathrm{CO}-\mathrm{X}]^{+}$ & 70 & 70 & 70 & - \\
\hline$[\mathrm{M}+\mathrm{H}-\mathrm{CO}-\mathrm{Pro}]^{+}$ & 72 & 86 & 120 & 136 \\
\hline$\left[\mathrm{M}+\mathrm{H}-\mathrm{NH}{ }_{3}-\mathrm{Pro}\right]^{+}$ & - & - & 131 & 147 \\
\hline
\end{tabular}




\section{Untersuchung ausgewählter Stämme aus arktischen und antarktischen Habitaten}

\subsection{Pseudoalteromonas sp. T268}

Der Stamm T268 wurde aus dem Darminhalt der antarktischen Krillart Euphausia superba isoliert. Im Dünnschichtchromatogramm des nach Kultivierung erhaltenen Rohextraktes fielen zwei Zonen auf, die weder UV-löschend noch fluoreszierend waren. Nach Besprühen mit Anisaldehyd und Erwärmung zeigte eine der Zonen eine blaue Anfärbung, welche auf das Vorliegen von Fettsäuren hindeutete. Die andere Zone färbte sich weder mit Anisaldehyd noch mit Ehrlichs Reagenz an. Im biologischen Screening zeigte der Rohextrakt des Stammes keine auffälligen Aktivitäten.

\subsubsection{Bestimmung der Taxonomie}

T268 wächst in Form beiger, glänzender Kolonien. Die 16S rRNA-Sequenz zeigte 99 \% Homologie zu Pseudoalteromonas sp. Der Stamm ist Gram-negativ und psychrotolerant, d.h. er wächst sowohl bei Temperaturen um $1^{\circ} \mathrm{C}$ als auch oberhalb von $30^{\circ} \mathrm{C}$, wobei das Wachstum stark vom Gehalt an Seewassersalzen im Nährmedium abhängt. T268 ist Oxidase- und Katalase-positiv, vermag Nitrat nicht zu reduzieren und zeigt keine Bildung von Schwefelwasserstoff. Gelatine, Chitin und Casein können abgebaut werden, Cellulose und Stärke hingegen nicht. Der Stamm zeigt weder $\beta$-Galactosidase- noch Arginin-, Lysinsowie Ornithin-Decarboxylaseaktivität. Im SFN-Biolog Verwertungstest werden die fettähnlichen Substanzen Tween 40 und Tween 80 metabolisiert.

\subsubsection{Isatin}

Die orange-farbene Verbindung 19 fiel im Dünnschichtchromatogramm $\left(R_{f}=0.35\right.$, $\mathrm{CH}_{2} \mathrm{Cl}_{2} / \mathrm{MeOH}$ 95:5) durch die intensive Eigenfarbe sowie UV-Löschung bei $254 \mathrm{~nm}$ auf. Die Substanz ging beim Besprühen mit Anisaldehyd in der Wärme einen Farbumschlag von Orange nach Hellgelb ein.

Das ${ }^{1} \mathrm{H}-\mathrm{NMR}-$ Spektrum zeigte nur Resonanzen im Aromatenbereich. Die Signale höherer Ordnung bei $\delta=7.6,7.57,7.14$ und 6.90 konnten einem 1,2-disubstituierten Benzolring zugeordnet werden. Weiterhin war ein breites Signal bei $\delta=7.81$ sichtbar, das auf das 
Vorliegen einer OH- oder NH-Gruppe hindeutete. Eine auf dem EI-Fragmentierungsmuster basierende Datenbanksuche mit NIST zeigte an, dass Isatin (19) isoliert wurde. Ein Vergleich der spektroskopischen Daten mit denen von authentischem Isatin (19) ergab vollständige Übereinstimmung und bestätigte somit die Zuordnung.<smiles>O=C1Nc2ccccc2C1=O</smiles>

19

Isatin (19) zeigt antifungische Aktivität gegen den Pilz Lagenidium callinectes, der Infektionen bei extern ausgebrüteten Embryonen der Shrimpart Palaemon macrodactylus hervorruft. In einer von FENICAL und Mitarbeitern aufgestellten Symbiose-Theorie ${ }^{53}$ geht man davon aus, dass eine Isatin (19) produzierende Alteromonaden-Art, die sich auf der Oberfläche von gesunden Embryonen befindet, essentiell für deren Überleben ist.

\subsubsection{3-Hydroxy-3-acetonyloxindol}

Der farblose Feststoff 20 war im Dünnschichtchromatogramm $\left(R_{f}=0.23, \mathrm{CH}_{2} \mathrm{Cl}_{2} / \mathrm{MeOH}\right.$ 95:5) durch UV-Löschung bei $254 \mathrm{~nm}$ auffällig und färbte sich beim Besprühen mit Anisaldehyd violett und mit Ehrlichs Reagenz grün an.

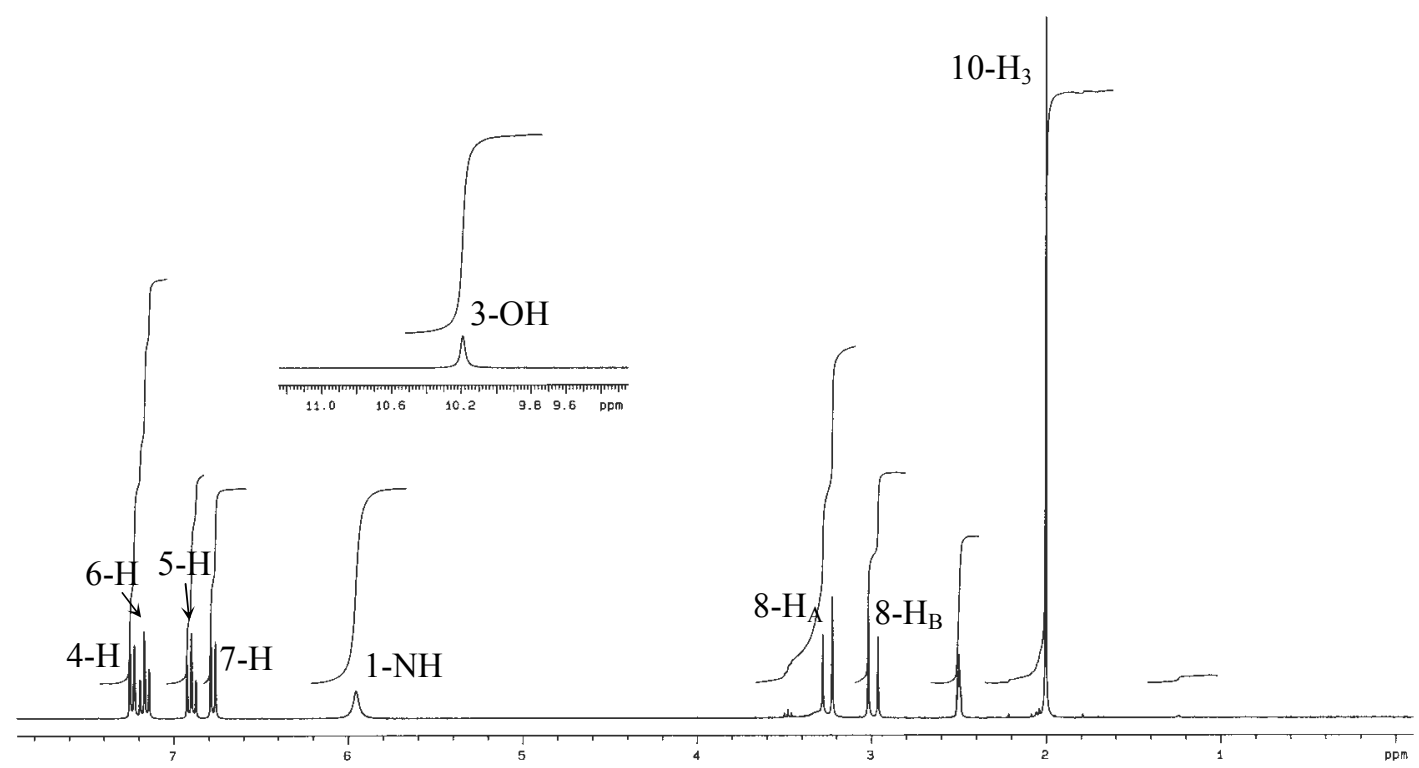

Abbildung $22{ }^{1} \mathrm{H}-\mathrm{NMR}-\mathrm{Spektrum}\left(300 \mathrm{MHz},\left[\mathrm{D}_{6}\right] \mathrm{DMSO}\right)$ von 20. 
Verbindung 20 zeigte im EI-Massenspektrum das Ion höchster Masse bei $m / z=205$. Dem ${ }^{1}$ H-NMR-Spektrum waren im Aromatenbereich vier Signale bei $\delta=7.23,7.16,6.90,6.77$ zu entnehmen, die einem ortho-disubstituierten Benzolring zuzuordnen waren. Weiterhin waren zwei breite Signale bei $\delta=10.19$ und 5.96 erkennbar, die auf OH- bzw. NH-Gruppen im Molekül hindeuteten, sowie eine Methylgruppe bei $\delta=2.00$. Zusätzlich waren Resonanzen bei $\delta=3.25$ und 2.98 sichtbar, welche über eine geminale Kopplung wechselwirkten und das Vorliegen einer diastereotopen Methylengruppe anzeigten.

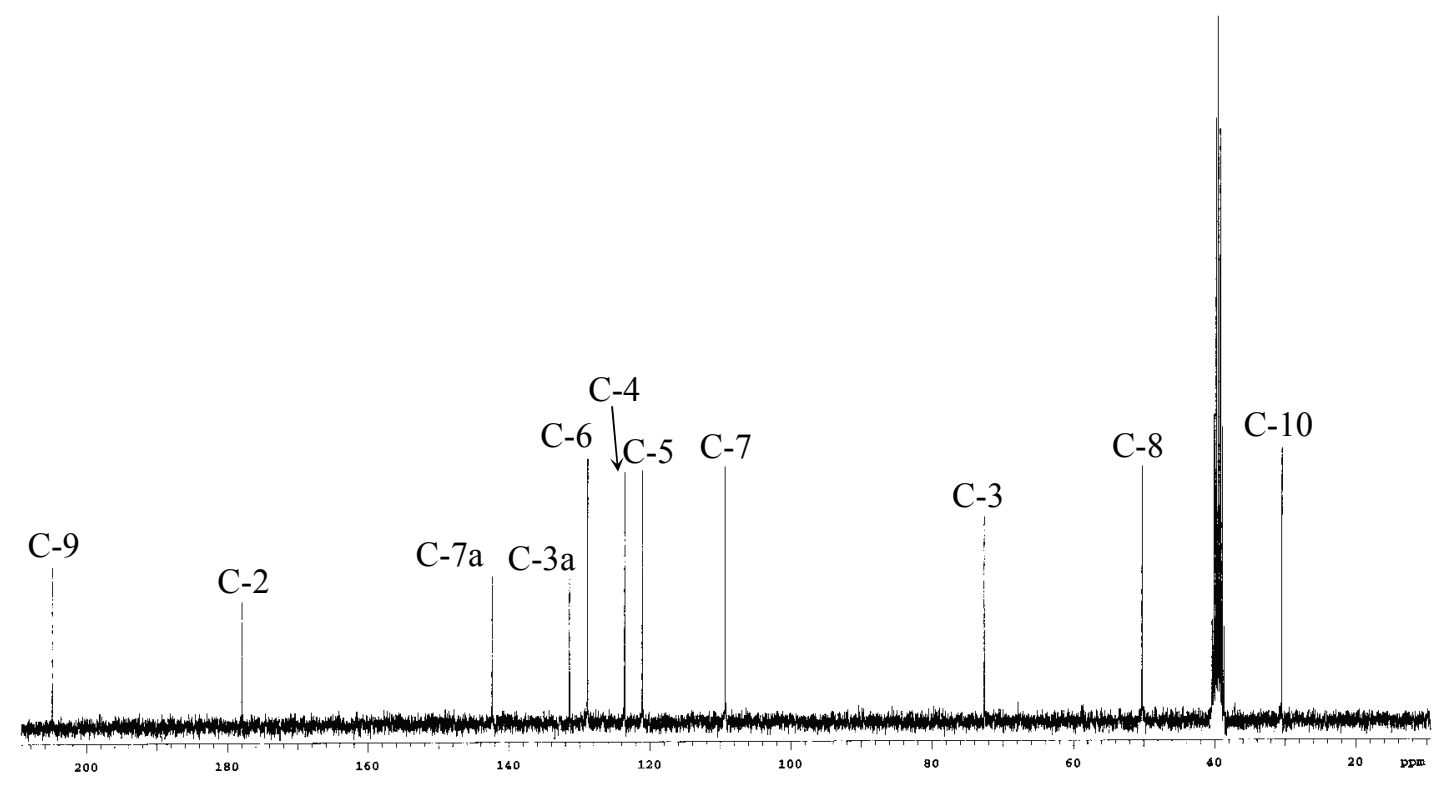

Abbildung $23{ }^{13} \mathrm{C}-\mathrm{NMR}-S p e k t r u m\left(300 \mathrm{MHz},\left[\mathrm{D}_{6}\right] \mathrm{DMSO}\right)$ von 3-Hydroxy-3-acetonyloxindol (20).

Das ${ }^{13} \mathrm{C}$-NMR-Spektrum zeigte ein Signal bei $\delta=205.0$, das für eine Ketogruppe sprach, ein Signal für eine Carbonylgruppe von Estern bzw. Amiden bei $\delta=178.0$ und sechs Signale im Aromaten- bzw. Olefinbereich bei $\delta=142.4-109.3$. Eine Datenbanksuche mit diesen Informationen in AntiBase ${ }^{30}$ führte zu 3-Hydroxy-3-acetonyloxindol (20). Die Verbindung wurde zur Strukturabsicherung ausgehend von Isatin und Aceton synthetisiert. Das erhaltene Produkt stimmte in allen NMR-spektroskopischen Daten mit dem isolierten Naturstoff 20 überein. Auffällig war der vergleichsweise niedrige Drehwert der isolierten Verbindung, welcher bei $+1.9^{\circ}(\mathrm{c}=1 \mathrm{mg} / \mathrm{ml}, \mathrm{MeOH})$ lag. Nach Trennung mittels einer chiralen HPLC-Säule stellte sich heraus, dass es sich um ein 1:1-Gemisch aus beiden enantiomeren Formen handelte. Neben einer enzymkatalysierten Biosynthese kann aufgrund des Vorliegens als Racemat die Möglichkeit nicht ausgeschlossen werden, dass 20 ein

\footnotetext{
* Für die Synthese von 3-Hydroxy-3-acetonyloxindol danke ich Marc Corbella.
} 
Artefakt ist, welches während der Aufarbeitung über eine Aldol-Reaktion aus Isatin und Aceton entsteht.

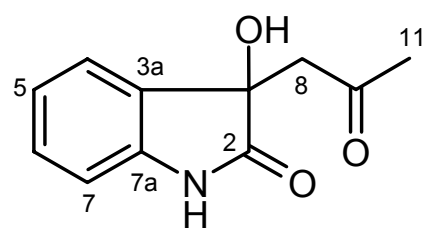

20

3-Hydroxy-3-acetonyloxindol (20) ist bereits seit längerem als Syntheseprodukt bekannt. Von der Arbeitsgruppe FENICAL wurde es erstmals als Naturstoff isoliert.

\subsection{4-Hydroxy-3-(1-propanonyl)oxindol}

Der im Dünnschichtchromatogramm $\left(R_{f}=0.25, \mathrm{CH}_{2} \mathrm{Cl}_{2} / \mathrm{MeOH}\right.$ 95:5) bei $254 \mathrm{~nm} \mathrm{UV-}$ löschende weiße Feststoff 21 färbte sich nach Besprühen mit Anisaldehyd grün und mit Ehrlichs Reagenz rot an.

Im EI-Massenspektrum wies 21 das Ion höchster Masse bei $m / z=219$ auf. Im Vergleich mit dem ${ }^{1}$ H-NMR-Spektrum von 20 zeigte 21 dieselben Signale im Aromatenbereich sowie für die diastereotope Methylengruppe. Es fehlte jedoch die Resonanz der Methylgruppe bei $\delta=2.21$. Stattdessen traten zwei zusätzliche Signale für eine Methylengruppe bei $\delta=2.44$ und eine Methylgruppe bei $\delta=1.00$ auf, die eine Ethyleinheit bilden. Verbindung 21 konnte somit als 3-Hydroxy-3-(1-propanonyl)oxindol (21) identifiziert werden. Diese Substanz ist bis jetzt weder als Naturstoff noch als Syntheseprodukt bekannt. Der Drehwert von 21 lag bei $+10.3^{\circ}$, die Trennung mittels einer chiralen HPLC-Säule lieferte die beiden Enantiomere im Verhältnis 17:20. Da ein Isomeres hier im Überschuss vorliegt, kann der Schluss gezogen werden, dass es sich bei beiden Enantiomeren um Naturstoffe handelt.<smiles>CCC(=O)CC1(O)C(=O)Nc2ccccc21</smiles>

21<smiles>O=C(O)C(=O)CC1(O)CNc2ccccc21</smiles>

22 
Der zu Verbindung 20 bzw. 21 ähnliche Naturstoff 22 wurde im Arbeitskreis ZEECK aus Halomonas sp. RK377 isoliert. ${ }^{54}$ Dieser 3-(3-Hydroxy-2,3-dihydro-1H-indol-3-yl)-2-oxopropionsäure (22) produzierende Stamm wurde aus einer in Norddeich-Mole gesammelten Wasserprobe isoliert. Bei den Convolutamydinen A (23), ${ }^{55}$ B (24), C (25) und D (26) handelt es sich um eine Reihe bromierter Hydroxyoxindole, ${ }^{56}$ welche von H. P. ZHANG et al. aus dem marinen Bryozoen Amathia convoluta isoliert wurden. Sie zeigen inhibitorische Aktivität gegenüber der Differenzierung von menschlichen Promyelozytenleukämie-Zellen der HL-60-Linie. ${ }^{57}$<smiles>[R]C1(O)C(=O)Nc2cc(Br)cc(Br)c21</smiles>

23: $\mathrm{R}=\mathrm{CH}_{2} \mathrm{COCH}_{3}$

24: $\mathrm{R}=\mathrm{CH}_{2} \mathrm{CH}_{2} \mathrm{Cl}$

25: $\mathrm{R}=\mathrm{CH}_{3}$

26: $\mathrm{R}=\mathrm{CHCH}_{2}$

\subsubsection{Ubichinon Q8}

Die sich mit Anisaldehyd in der Wärme violett anfärbende Substanz 27 fiel als gelbes Ö1 an, das im Dünnschichtchromatogramm eine bei $254 \mathrm{~nm}$ UV-Licht löschende Zone $\left(R_{f}=\right.$ 0.93, $\mathrm{CH}_{2} \mathrm{Cl}_{2} / \mathrm{MeOH}$ 95:5) zeigte.

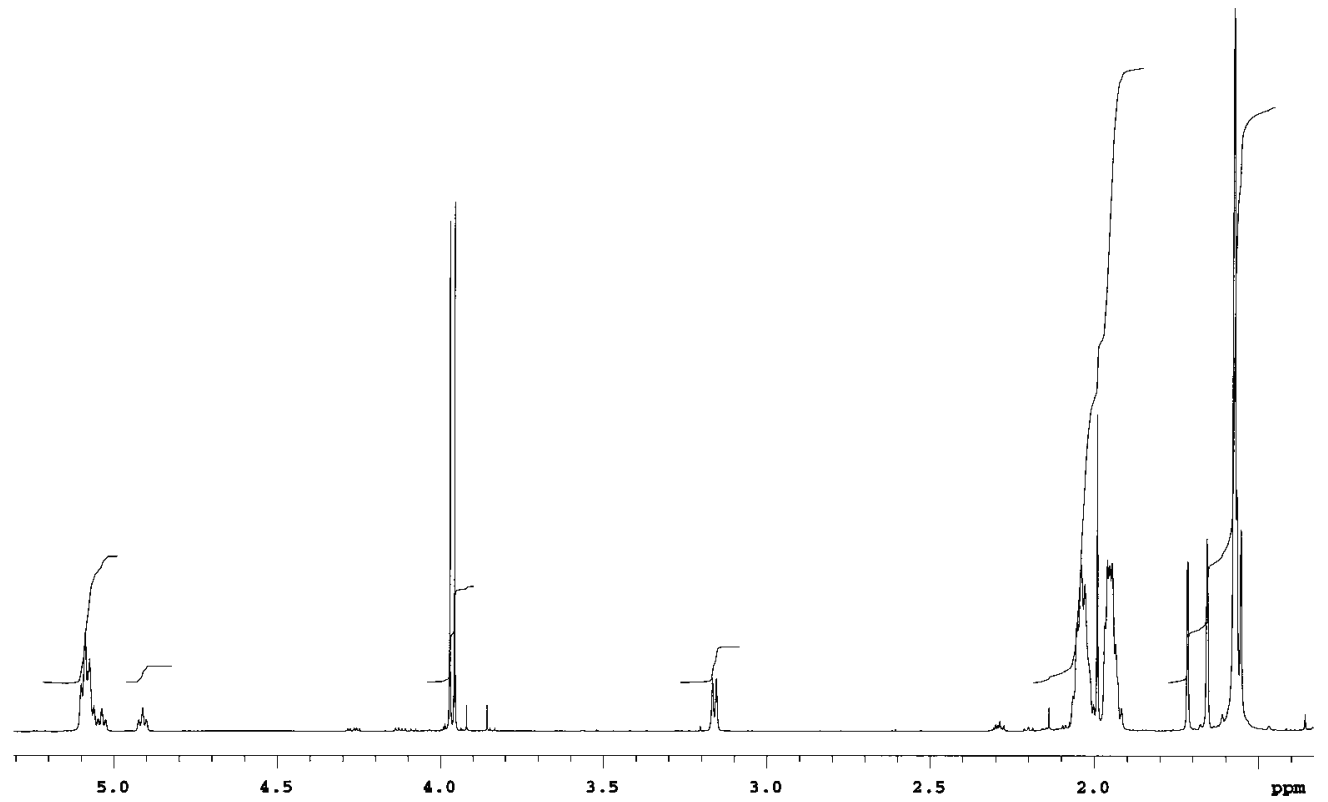

Abbildung $24{ }^{1} \mathrm{H}-\mathrm{NMR}-S p e k t r u m\left(600 \mathrm{MHz}, \mathrm{CDCl}_{3}\right)$ von Ubichinon Q8 (27). 
Im ${ }^{1}$ H-NMR-Spektrum waren acht Methingruppen $(\delta=5.09$ - 4.91), zwei Methoxygruppen $(\delta=3.97$, 3.96), fünfzehn Methylengruppen $(\delta=3.16,2.05-1.94)$ sowie zehn Methylgruppen $(\delta=1.99(3 \mathrm{H}), 1.72(3 \mathrm{H}), 1.66(3 \mathrm{H}), 1.56(21 \mathrm{H})) \mathrm{zu}$ erkennen. Im Aromatenbereich hingegen wurde kein Signal beobachtet. Das ESI-Massenspektrum zeigte ein Ion bei $m / z=749[\mathrm{M}+\mathrm{Na}]^{+}$. Eine Suche in AntiBase ${ }^{30}$ mit diesen Informationen lieferte Ubichinon Q8 (27) als mögliche Struktur, deren Vorliegen durch NMR-Datenvergleich mit in unserer Arbeitsgruppe vorhandenen Spektren bestätigt wurde.<smiles>COC1=C(OC)C(=O)C(C/C=C(\C)C/C=C(\C)CC/C=C(\C)CC/C=C(\C)CC/C=C(\C)CC/C=C(\C)CC/C=C(\C)CCC=C(C)C)=C(C)C1=O</smiles>

27

\subsubsection{Ubichinon KQ5}

Die ölige Substanz 28 zeigte im Dünnschichtchromatogramm eine gelbe Zone, die UVLicht der Wellenlänge $254 \mathrm{~nm}$ löschte und sich mit Anisaldehyd in der Wärme blau anfärbte. Die Polarität von $28\left(R_{f}=0.65, \mathrm{CH}_{2} \mathrm{Cl}_{2} / \mathrm{MeOH} 95: 5\right)$ war deutlich höher als diejenige von Verbindung 27.

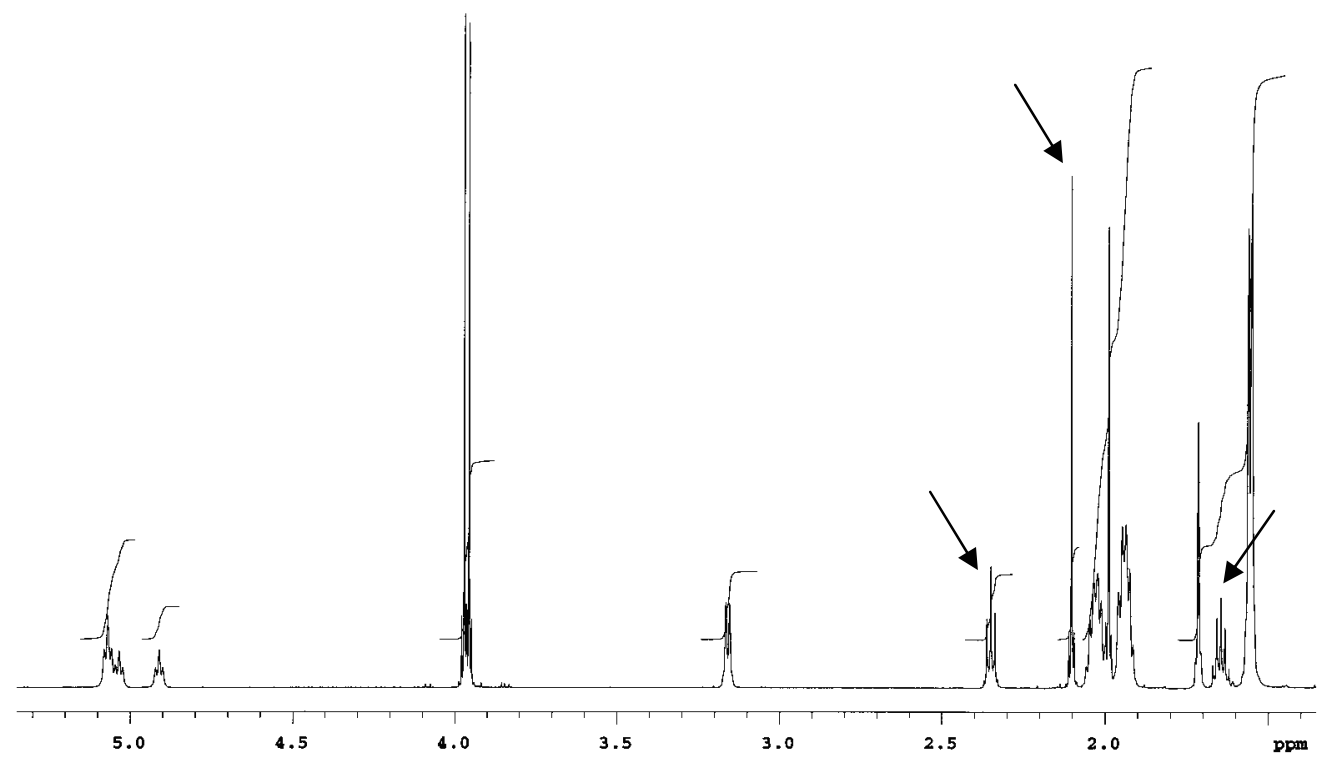

Abbildung $25{ }^{1} \mathrm{H}-\mathrm{NMR}-$ Spektrum $\left(600 \mathrm{MHz}, \mathrm{CDCl}_{3}\right)$ von 28. Im Vergleich mit 27 zusätzlich vorhandene Signale sind mit einem Pfeil markiert. 


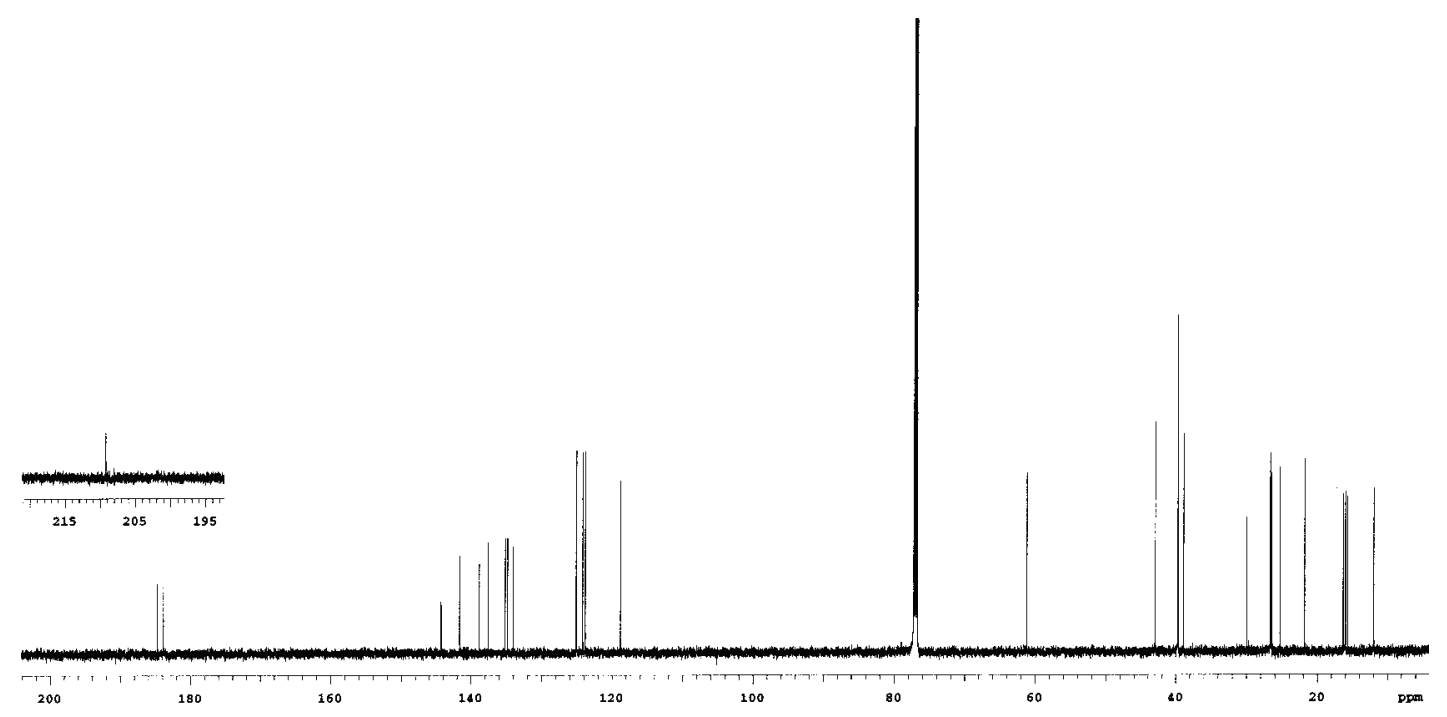

Abbildung $26{ }^{13} \mathrm{C}-\mathrm{NMR}-\mathrm{Spektrum}\left(300 \mathrm{MHz}, \mathrm{CDCl}_{3}\right)$ von 28.

Im ESI-Massenspektrum zeigte ein Ion bei $m / z=547[\mathrm{M}+\mathrm{Na}]^{+}$, aus dem per Hochauflösung die Summenformel $\mathrm{C}_{33} \mathrm{H}_{48} \mathrm{O}_{5}$ abgeleitet werden konnte. Im Vergleich mit dem ${ }^{1} \mathrm{H}$ NMR-Spektrum von 27 wies Verbindung 28 zusätzliche Signale für zwei Methylengruppen bei $\delta=2.35$ und 1.64 sowie eine Methylgruppe bei $\delta=2.10$ auf. Es fehlte jedoch das Singulett für die Methylgruppe bei $\delta=1.66$. Im ${ }^{13} \mathrm{C}-\mathrm{NMR}-$ Spektrum war das Signal einer zusätzlichen Ketogruppe bei $\delta=209.2$ auffällig. Das H,H-COSY-Spektrum zeigte die Kopplung der Methylenprotonen untereinander sowie mit einem Signal für eine weitere Methylengruppe bei $\delta \sim 1.9$ an, welches mit den Resonanzen der Isopren-Methylengruppen überlagerte. Somit konnte das in Abbildung 39 gezeigte Strukturelement abgeleitet werden. Die im HMBC-Experiment beobachteten Korrelationen erlaubten die Verknüpfung der gefundenen Fragmente. 


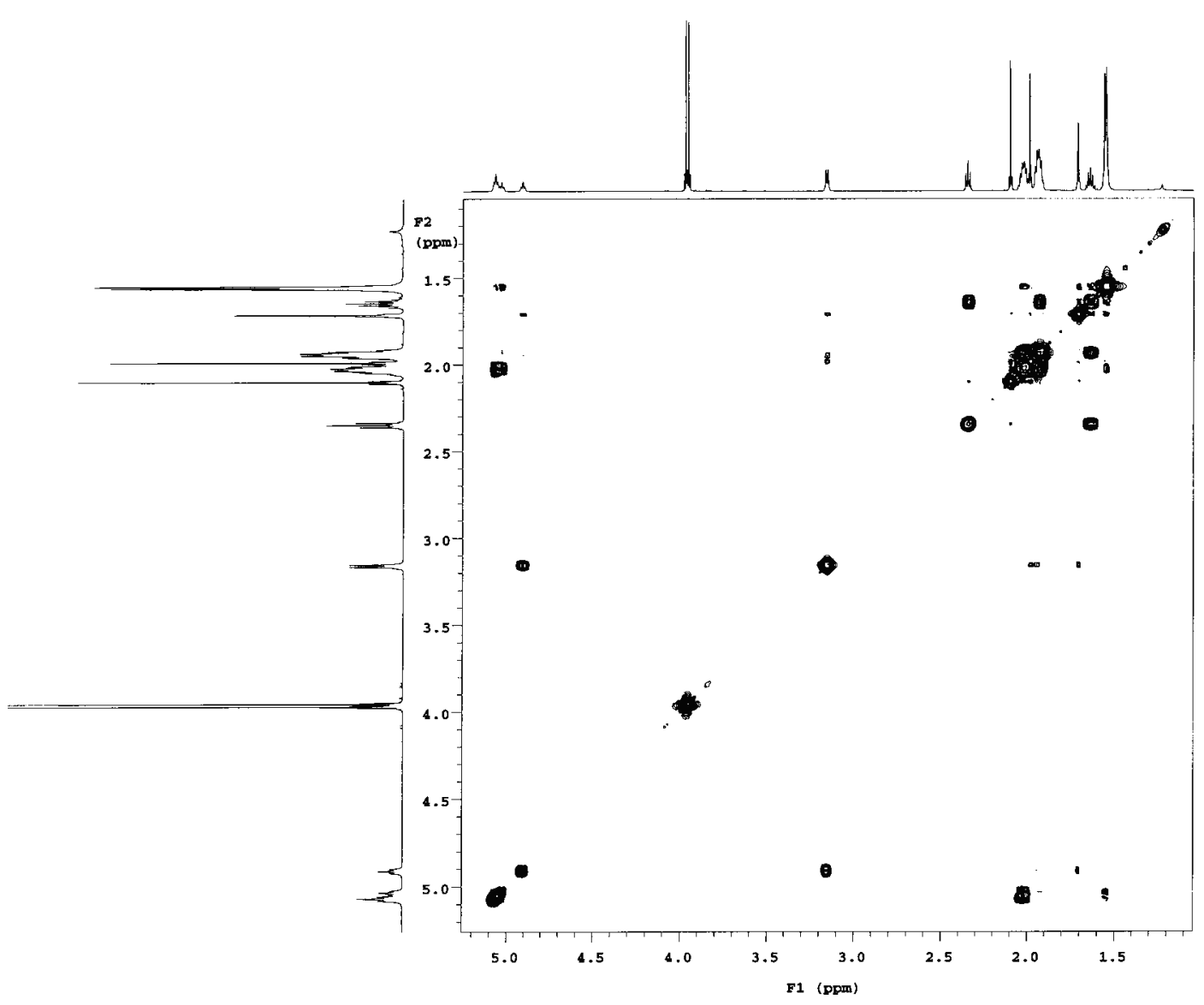

Abbildung 27 H,H-COSY-Spektrum (600 MHz, CDCl 3 ) von 28.<smiles>C1CCCCCC1</smiles>

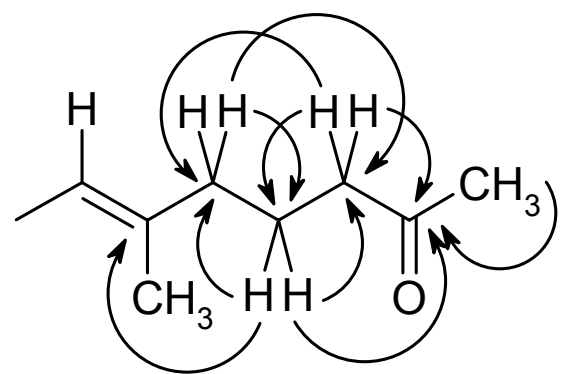

Abbildung 28 Für die Strukturzuordnung entscheidende H,H-COSY- und HMBC-Korrelationen von 28. 


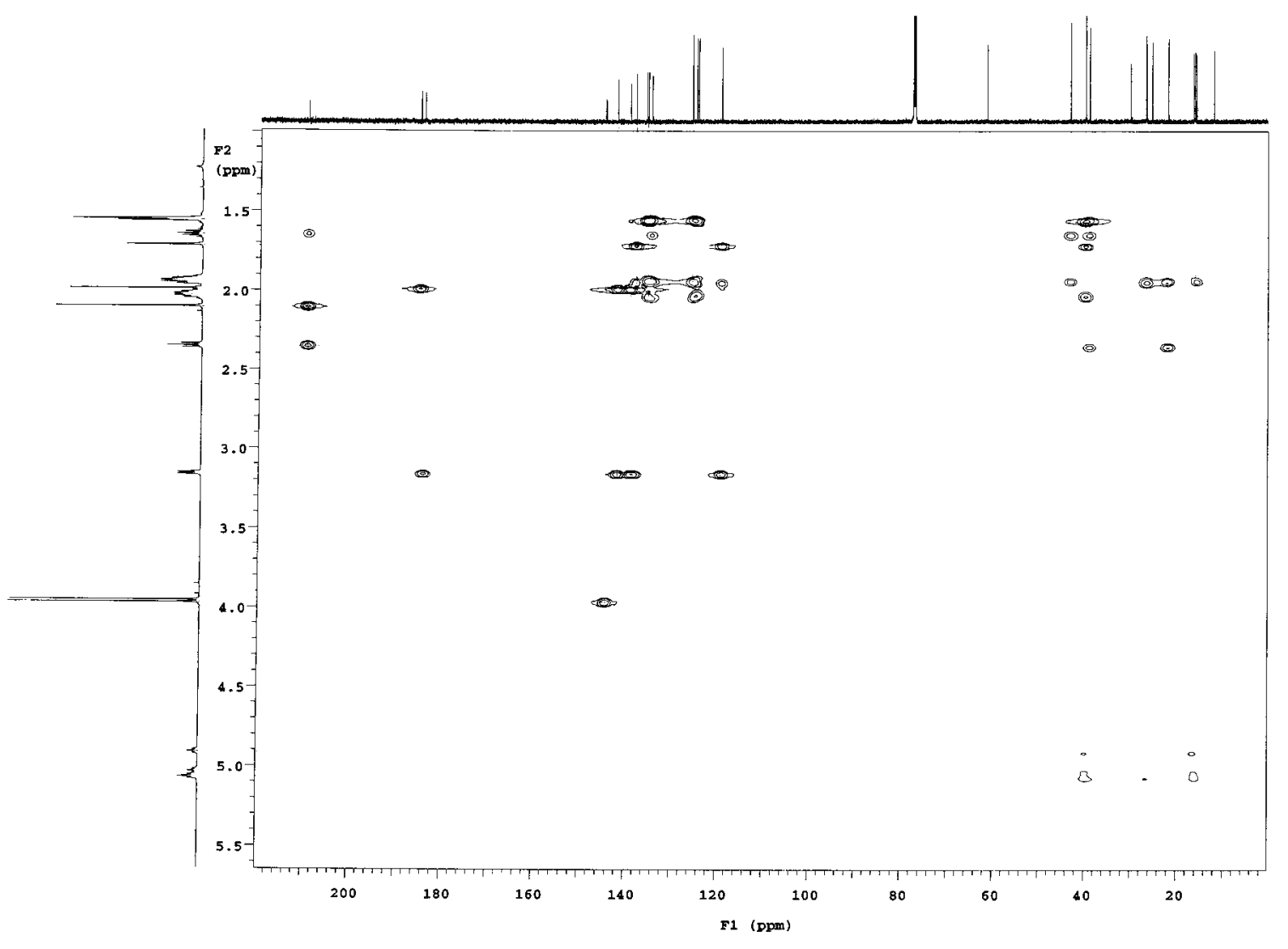

Abbildung 29 HMBC-Spektrum $\left(600 \mathrm{MHz}, \mathrm{CDCl}_{3}\right)$ von 28.<smiles>COC1=C(OC)C(=O)C(C/C=C(\C)C/C=C(\C)CC/C=C(\C)CC/C=C(\C)CCCC(C)=O)=C(C)C1=O</smiles>

Die weitere Auswertung der NMR-Daten führte zu dem Ergebnis, dass es sich um das Ubichinonderivat $\mathbf{2 8}$ handeln musste, in dem formal eine der terminalen Methylgruppen gegen eine Sauerstofffunktion ersetzt worden war. Damit wurde 28 als Ubichinon KQ5 identifiziert, wobei es sich um einen neuen Typ von Prenylchinonen handelt, der bislang weder als Naturstoff noch als Syntheseprodukt beschrieben worden ist. Gewisse Ähnlichkeiten zeigen lediglich die Trisporine und Trisporsäuren aus dem Pilz Blakeslea trispora, wie etwa Trisporin B (29). ${ }^{58}$ 
<smiles>CC(=O)CCC=C(C)C=CC1=C(C)C(=O)CCC1(C)C</smiles>

29

Ob 28 tatsächlich durch Demethylierung und Oxidation eines Pentaprenyl-Restes nach einem normalen Ubichinon-Biosyntheseverlauf ${ }^{59}$ entsteht oder vielmehr durch Addition von Geranyl-geranylaceton an ein 2,3-Dimethoxy-5,6-dimethylchinon gebildet wird (siehe Abbildung 30), muss anhand von Fütterungsexperimenten mit markierten Vorläufermolekülen geprüft werden. Diese konnten im Rahmen der vorliegenden Arbeit aus Zeitgründen nicht durchgeführt werden.<smiles>COC1=C(OC)C(=O)C(C)=C(C)C1=O</smiles>

Abbildung 30 Biosynthesevorschläge von Verbindung 28. 


\subsubsection{Ubichinon KQ2}

Die Substanz 30 fiel auf dem Dünnschichtchromatogramm durch ihre gelbe Farbe und UVLöschung sowie eine grüne Anfärbung mit Anisaldehyd/Schwefelsäure auf. Im Vergleich mit 27 und 29 zeigte sie eine höhere Polarität $\left(R_{f}=0.56, \mathrm{CH}_{2} \mathrm{Cl}_{2} / \mathrm{MeOH} 95: 5\right)$.

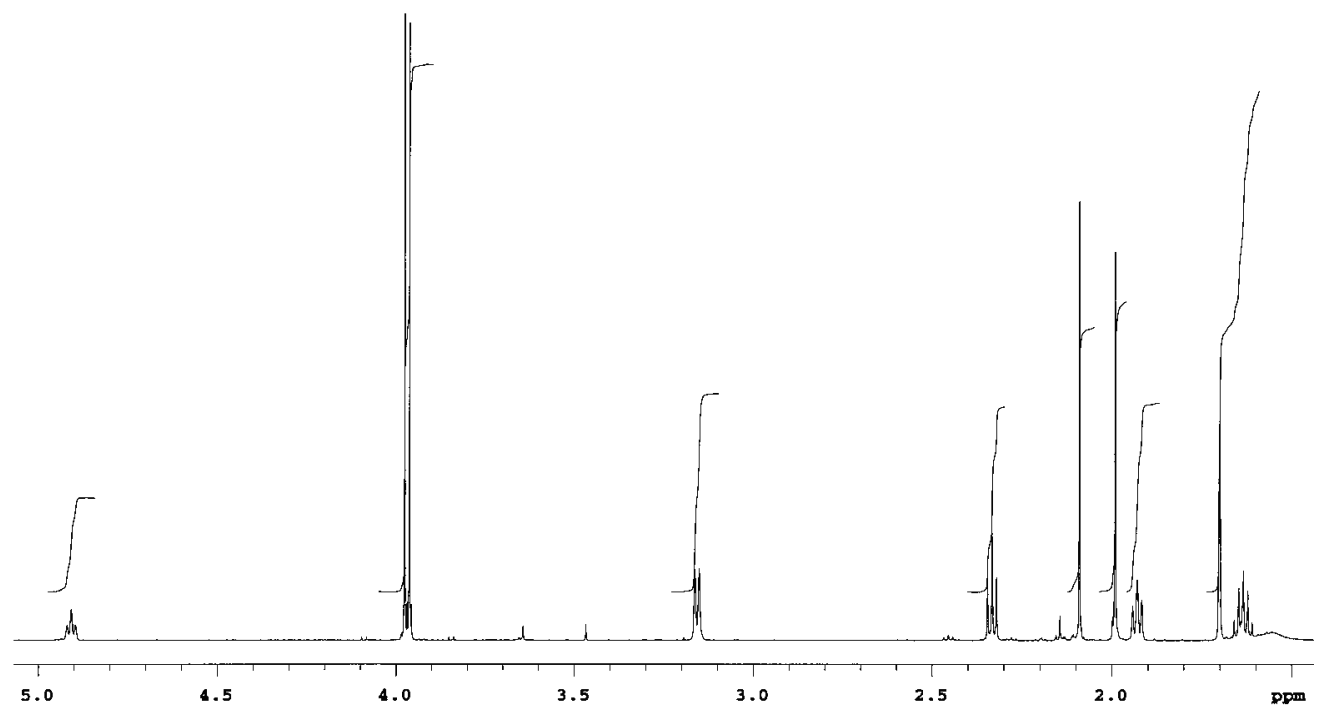

Abbildung $31{ }^{1} \mathrm{H}-\mathrm{NMR}-$ Spektrum $\left(600 \mathrm{MHz}, \mathrm{CDCl}_{3}\right)$ von 30.

Im ESI-Massenspektrum zeigte 30 ein Ion bei $m / z=343[\mathrm{M}+\mathrm{Na}]^{+}$. Aus dem ${ }^{1} \mathrm{H}-\mathrm{NMR}-$ Spektrum sind Resonanzen für eine Methingruppe bei $\delta=4.91$, zwei Methoxygruppen bei $\delta=3.98,3.96$, vier Methylengruppen bei $\delta=3.16,2.33$, 1.93, 1.64 sowie drei Methylgruppen bei $\delta=2.09,1.99,1.70$ zu erkennen. Das ${ }^{13} \mathrm{C}-\mathrm{NMR}-S p e k t r u m$ zeigte die Existenz von 28 Kohlenstoffatomen an, wobei anhand der typischen chemischen Verschiebungen eine Ketogruppe bei $\delta=208.9$ sowie zwei einem Chinonsystem zuzuordnende Carbonylsignale bei $\delta=184.7,183.9 \mathrm{zu}$ sehen waren. Im Vergleich mit den NMR-Daten von 29 fehlten die Signale für die Polyisopren-Seitenkette. Mit Hilfe von H,H-COSY- und HMBC-Experimenten konnten die Strukturelemente abgeleitet und die gefundenen Fragmente verknüpft werden. Diese Verbindung war bereits von der Arbeitsgruppe FENICAL aus dem Cyanobakterium Microcoleus lyngbyaceus isoliert und identifiziert worden. ${ }^{60}$<smiles></smiles> 
Von $p$-Chinon abgeleitete Strukturen mit Isopren-Seitenkette, deren Länge stark von der produzierenden Spezies abhängt, spielen aufgrund ihrer Redoxaktivität eine wichtige Rolle im pflanzlichen und tierischen Metabolismus. Sie eignen sich folglich zur taxonomischen Charakterisierung unterschiedlicher Organismen. ${ }^{61}$ Im Stoffwechsel von Menschen und Tabakpflanzen findet man Chinone mit zehn Isopren-Einheiten, während die von Nagetieren, E. coli und Hefe (Saccharomyces cerevisiae) produzierten aus sechs, acht oder neun Einheiten aufgebaut sind. Die Prenylchinone teilt man in zwei Gruppen ein, Ubichinone (Coenzym Q) und Menachinone (Vitamin $\mathrm{K}_{2}$ ) ein. Bei den meisten Gram-positiven sowie bei anaerob lebenden Gram-negativen Bakterien findet man ausschließlich Menachinone, während die meisten aeroben Gram-negativen Bakterien nur Ubichinone aufbauen. Hin und wieder findet man bei beiden Vertretern partiell gesättigte Polyisoprenyl-Seitenketten sowie andere Chinon-Derivate wie Chlorobiumchinone, $\alpha$-Tocopherolchinone, Rhodochinone, Epoxyubichinone und schwefelhaltige Caldariellachinone. ${ }^{61}$

Ubichinon (CoQ) wurde 1961 gleichzeitig von MORTON ${ }^{62}$ und CRANE $^{63}$ gefunden. Eine wichtige Eigenschaft von Ubichinonen ist ihre Hydrophobizität, welche Mobilität innerhalb der Zellmembran gewährleistet und für eine freie Wanderung zwischen den zu reduzierenden bzw. oxidierenden Spezies ermöglicht. Ubichinon spielt eine wichtige Rolle als Wasserstoffüberträger zwischen NADH und dem Enzym Succinat-Dehydrogenase sowie dem Cytochrom-System. Die Reduktion von CoQ beinhaltet eine Übertragung von zwei Elektronen sowie die Addition von zwei Protonen und führt zur Bildung von Ubichinol. Im umgekehrten Oxidations-Prozess erfolgt die Bildung von Ubichinon unter Freisetzung von zwei Elektronen bzw. Protonen. ${ }^{64}$

\subsubsection{B-Carbolin-Derivate}

\section{Norharman}

Der weiße Feststoff 31a fiel im Dünnschichtchromatogramm $\left(R_{f}=0.37, \mathrm{CH}_{2} \mathrm{Cl}_{2} / \mathrm{MeOH}\right.$ 9:1) durch UV-Löschung bei $254 \mathrm{~nm}$ sowie durch starke hellblaue Fluoreszenz bei $366 \mathrm{~nm}$ auf. Die Verbindung war weder mit Anisaldehyd/Schwefelsäure noch mit Ehrlichs Reagenz anfärbbar.

Im ${ }^{1}$ H-NMR-Spektrum waren ausschließlich Resonanzen im Aromatenbereich detektierbar. Die Signale bei $\delta=8.05,7.62,7.53$ und 7.25 konnten anhand ihres charakteristischen Kopplungsmusters einem 1,2-disubstituierten Benzolring zugeordnet werden. Das bei 
$\delta=10.78$ erscheinende breite Singulett deutete auf das Vorliegen einer OH- bzw. NHGruppe im Molekül hin. Ein weiteres Singulett bei $\delta=8.90$ sowie zwei Dubletts bei $\delta=8.35$ und 8.22 konnten jeweils einer aromatischen Methingruppe zugeschrieben werden. Im EI-Massenspektrum war das Ion höchster Masse bei $m / z=168$ sichtbar, dessen Fragmentierungsmuster mit dem von $\beta$-Carbolin 31a übereinstimmte. Dass es sich bei der isolierten Substanz tatsächlich um die auch unter dem Trivialnamen Norharman 31a bekannte Verbindung handelte, konnte durch ${ }^{1} \mathrm{H}-\mathrm{NMR}$-Datenvergleich mit einem in unserer Arbeitsgruppe vorhandenen Referenzspektrum von 31a bestätigt werden.<smiles>[R]c1nccc2c1[nH]c1ccccc12</smiles>

31a: $\mathrm{R}=\mathrm{H}$

31b: $\mathrm{R}=\mathrm{CH}_{3}$

\section{Harman}

Die Verbindung 31b war auf dem Dünnschichtchromatogramm hinsichtlich UV-Löschung und Anfärbeverhalten mit Anisaldehyd/Schwefelsäure identisch mit 31a, zeichnete sich aber durch eine höhere Polarität aus $\left(R_{f}=0.35, \mathrm{CH}_{2} \mathrm{Cl}_{2} / \mathrm{MeOH} 9: 1\right)$.

Der Vergleich mit dem ${ }^{1}$ H-NMR-Spektrum von 31a deutete auf das Vorhandensein desselben ß-Carbolin-Grundgerüsts hin. Es fehlte jedoch das Signal für ein aromatisches Proton bei $\delta=8.90$, dafür trat eine zusätzliche Methylgruppe bei $\delta=2.77$ auf. Das EI-Massenspektrum zeigte ein Ion höchster Masse bei $m / z=128$. Eine Datenbanksuche in AntiBase ${ }^{30}$ und anschließender Vergleich des ${ }^{1}$ H-NMR-Spektrums mit der in unserer Arbeitsgruppe vorhandenen Spektrensammlung führte zur Identifizierung von 31b als Harman.

Harman, ein typischer Pflanzenmetabolit, ${ }^{65}$ wurde bereits mehrfach als Stoffwechselprodukt bei Actinomyceten ${ }^{66}$ und Dinoflagellaten ${ }^{67}$ gefunden. 


\subsubsection{Glutarsäure-Derivate}

\section{Glutarsäure}

Bei dem Hauptmetaboliten des Stammes T268 handelte es sich um eine farblose Substanz, die weder UV-löschend noch fluoreszierend war und keine Farbreaktion beim Besprühen mit Anisaldehyd/Schwefelsäure sowie Ehrlichs Reagenz einging. Sie konnte lediglich als ausgedehnte farblose Zone im Dünnschichtchromatogramm $\left(R_{f}=0.25, \mathrm{CH}_{2} \mathrm{Cl}_{2} / \mathrm{MeOH}\right.$ 95:5) nach Anfärbung detektiert werden und wurde in einer Ausbeute von $6 \mathrm{mg} / \mathrm{l}$ isoliert.

Das ${ }^{1} \mathrm{H}-\mathrm{NMR}-\mathrm{Spektrum}$ zeigte zwei Signale für drei Methylengruppen bei $\delta=2.37$ und 1.85, die im Integralverhältnis 2:1 zueinander standen. Dies deutete auf das Vorliegen einer symmetrischen Struktur hin. Anhand der NMR-Daten und mittels des Fragmentierungsmusters im EI-Massenspektrum konnte die Substanz als Glutarsäure (32a) identifiziert werden.<smiles>[R]C(=O)CCCC([R])=O</smiles>

\section{Glutarsäuremonomethylester}

Die ebenfalls als farblose Flüssigkeit anfallende Verbindung 32b war auf dem Dünnschichtchromatogramm hinsichtlich UV- und Anfärbeverhalten mit Anisaldehyd/Schwefelsäure sowie Ehrlichs Reagenz identisch mit 32a zeichnete sich aber durch geringere Polarität $\left(R_{f}=0.42, \mathrm{CH}_{2} \mathrm{Cl}_{2} / \mathrm{MeOH} 95: 5\right)$ aus.

Im ${ }^{1} \mathrm{H}-\mathrm{NMR}-$ Spektrum waren drei Methylengruppen bei $\delta=2.44,2.42$ und $1.96 \mathrm{zu}$ erkennen. Zusätzlich konnte ein breites Singulett bei $\delta \sim 10$, das einer OH- bzw. NHGruppe zugeschrieben wurde, sowie eine Methoxygruppe bei $\delta=3.69$ detektiert werden. Das EI-Massenspektrum zeigte ein Ion höchster Masse bei $m / z=128$. Anhand des Fragmentierungsmusters und der ${ }^{1} \mathrm{H}-\mathrm{NMR}$-Daten konnte die Verbindung als Glutarsäuremonomethylester (32b) identifiziert werden. Es kann nicht ausgeschlossen werden, dass 32b im Zuge der chromatographischen Aufarbeitung durch Veresterung von Glutarsäure (32a) mit im Laufmittelgemisch vorhandenem Methanol gebildet wird. 


\subsubsection{Indolderivate}

\section{3-Indolylcarbonsäure}

Das farblose Öl 33 zeigte im Dünnschichtchromatogramm UV-Löschung bei $254 \mathrm{~nm}$ und färbte sich mit Anisaldehyd/Schwefelsäure orange und mit Ehrlichs Reagenz violett an $\left(R_{f}=0.44, \mathrm{CH}_{2} \mathrm{Cl}_{2} / \mathrm{MeOH} 9: 1\right)$, was auf ein Indol hinwies.

Im ${ }^{1} \mathrm{H}-\mathrm{NMR}-$ Spektrum waren lediglich aromatische Protonen sichtbar. Die Signale bei $\delta=8.09,7.40,7.16$ und 7.13 waren aufgrund des typischen Kopplungsmusters einem 1,2disubstituierten Phenylring zuzuordnen. Weiterhin trat ein Singulett für eine aromatische Methingruppe bei $\delta=7.88$ auf. Eine mit den aus Massen- und ${ }^{1} \mathrm{H}-\mathrm{NMR}-\mathrm{Spektrum}$ gewonnenen Informationen durchgeführte Suche in AntiBase ${ }^{30}$ lieferte 3-Indolylcarbonsäure (33) als mögliche Struktur. Ein Vergleich der ${ }^{1}$ H-NMR-Daten von 33 mit der Spektrensammlung ergab vollständige Übereinstimmung und bestätigte die Zuordnung.

Verbindung 33 ist auch unter den Namen Heteroauxin sowie Rhizopin bekannt und spielt als Wuchsstoff im Metabolismus von Pflanzen eine Rolle. ${ }^{68}$ Ebenso ist es als Metabolit mariner Mikroorganismen sowie halophiler Bakterien ${ }^{69}$ bekannt.

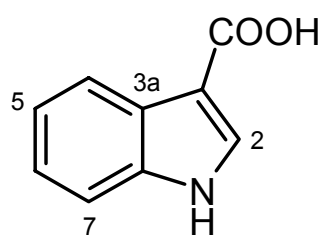

33

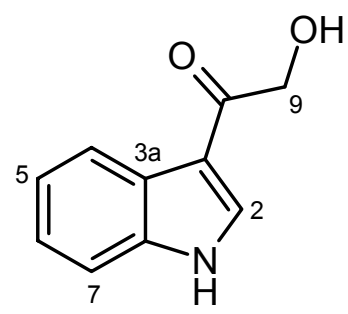

34

\section{3-Hydroxyacetylindol}

Der weiße Feststoff 34 fiel im Dünnschichtchromatogramm durch UV-Löschung bei $254 \mathrm{~nm}$ sowie durch hellrote Färbung beim Besprühen mit Ehrlichs Reagenz auf $\left(R_{f}=0.28, \mathrm{CH}_{2} \mathrm{Cl}_{2} / \mathrm{MeOH} 95: 5\right)$.

Der Vergleich des ${ }^{1}$ H-NMR-Spektrums mit dem von $\mathbf{3 3}$ zeigte, dass bei $\mathbf{3 4}$ wiederum ein Indolgerüst vorlag, jedoch trat eine zusätzliche Methylengruppe bei $\delta=4.72$ auf. Das EIMassenspektrum zeigte ein Ion höchster Masse bei $m / z=175$, welches ein für 3-Hydroxyacetylindol (34) typisches Fragmentierungsmuster aufwies. Ein Vergleich der Masse- und ${ }^{1}$ H-NMR-Daten mit den im Arbeitskreis vorhandenen Spektren von 34 bestätigte die Zuordnung. 


\subsubsection{Sonstige Metaboliten}

\section{Uracil}

Die polare Substanz 35 fiel im Dünnschichtchromatogramm $\left(R_{f}=0.25, \mathrm{CH}_{2} \mathrm{Cl}_{2} / \mathrm{MeOH}\right.$ 9:1) durch UV-Löschung bei $254 \mathrm{~nm}$ auf. Sie zeigte kein Anfärbeverhalten beim Besprühen mit Anisaldehyd und Ehrlichs Reagenz. Das ${ }^{1} \mathrm{H}-\mathrm{NMR}-$ Spektrum zeigte lediglich zwei Dubletts bei $\delta=7.38$ und 5.44. Diese Information führte in Kombination mit den aus dem EI-Massenspektrum erhaltenen Daten nach einer AntiBase-Suche ${ }^{30}$ rasch zu der im Arbeitskreis oft isolierten Verbindung Uracil (35). Ein Vergleich der Spektren mit den in unserer Sammlung vorhandenen zeigte eine vollständige Übereinstimmung.<smiles></smiles>

35<smiles>O=C(O)c1ccc(O)cc1</smiles>

36<smiles>OCCc1ccc(O)cc1</smiles>

37

\section{p-Hydroxybenzoesäure}

Die Verbindung 36 war im Dünnschichtchromatogramm als UV-löschende Zone $\left(R_{f}=\right.$ 0.49, $\mathrm{CH}_{2} \mathrm{Cl}_{2} / \mathrm{MeOH}$ 9:1) aufgefallen und wurde in einer Ausbeute von $2 \mathrm{mg} / \mathrm{l}$ als weißer Feststoff isoliert.

Das ${ }^{1}$ H-NMR-Spektrum von 36 war ähnlich dem von $\mathbf{3 5}$, jedoch wiesen die vorhandenen beiden Dubletts hier eine andere chemische Verschiebung auf. Mittels zusätzlicher Informationen aus dem EI-Massenspektrum konnte die Substanz als p-Hydroxybenzoesäure (36) identifiziert werden. Ein Vergleich der Masse- und ${ }^{1} \mathrm{H}-\mathrm{NMR}-\mathrm{Daten}$ mit denen der Spektrensammlung bestätigte die Struktur.

\section{Tyrosol}

Substanz 37 fiel als sich mit Anisaldehyd/Schwefelsäure lila anfärbende Zone $\left(R_{f}=0.44\right.$, $\mathrm{CH}_{2} \mathrm{Cl}_{2} / \mathrm{MeOH}$ 9:1) im Dünnschichtchromatogramm auf und konnte als weißer Feststoff isoliert werden.

Das ${ }^{1}$ H-NMR-Spektrum zeigte im Aromatenbereich zwei Dubletts, die für je zwei Protonen integrierten. Dies wies auf das Vorliegen eines 1,4-disubstituierten Aromaten hin. 
Zusätzlich waren zwei Tripletts für benachbarte Methylengruppen bei $\delta=7.10$ und 6.78 detektierbar. Die abgeleiteten Strukturfragmente führten zusammen mit den Informationen aus dem EI-Massenspektrum zu $p$-Hydroxyphenylethanol (37).

\section{Vanillinsäure}

Bei Verbindung 38 handelte es sich um einen weißen Feststoff, der auf dem Dünnschichtchromatogramm UV-Löschung zeigte und mit Anisaldehyd eine hellblaue Farbreaktion einging.

Im ${ }^{1}$ H-NMR-Spektrum waren drei Signale im Aromatenbereich zu sehen, die anhand ihres charakteristischen Kopplungsmusters $\mathrm{zu}$ einem 1,3,4-trisubstituierten Aromat gehören mussten. Zusätzlich konnte ein Signal für eine Methoxygruppe bei $\delta=3.89$ beobachtet werden. Das EI-Massenspektrum zeigte ein Ion höchster Masse bei $\mathrm{m} / \mathrm{z}=168$. Eine mit diesen Informationen durchgeführte Datenbanksuche in AntiBase ${ }^{30}$ ergab Vanillinsäure (38) als Strukturvorschlag. Ein Vergleich der ${ }^{1}$ H-NMR-Daten mit denjenigen aus der Spektrensammlung bestätigte die Zuordnung.<smiles>COc1cc(C(=O)O)ccc1O</smiles>

38<smiles>COc1cc(CCC(=O)O)ccc1O</smiles>

39

\section{4-Hydroxy-3-methoxy-phenyl-propionsäure}

Der schwach UV-löschende weiße Feststoff 39 zeigte im Dünnschichtchromatogramm nach Besprühen mit Anisaldehyd eine hellrote Färbung.

Die Analyse des ${ }^{1} \mathrm{H}-\mathrm{NMR}-$ Spektrums von 39 ergab, dass ebenfalls ein 1,3,4-trisubstituierter, methoxylierter Aromat vorlag. Im Vergleich mit Verbindung 38 waren jedoch zwei zusätzliche Signale für benachbarte Methylengruppen detektierbar. Das EI-Massenspektrum zeigte ein Ion höchster Masse bei $m / z=196$, was einem Unterschied von zwei Methylengruppen bezogen auf $\mathbf{3 8}$ entsprach. Eine Suche in AntiBase ${ }^{30}$ mit nachfolgendem Spektrenvergleich ergab, dass 4-Hydroxy-3-methoxy-phenyl-propionsäure (39) isoliert wurde. 


\subsection{Salegentibacter holothuriorum $\mathbf{T} 436$}

Der Stamm T436 wurde aus mehrjährigem arktischem Eis isoliert. Der Rohextrakt fiel im Dünnschichtchromatogramm $\left(\mathrm{CH}_{2} \mathrm{Cl}_{2} / \mathrm{MeOH}\right.$ 9:1) durch eine Reihe gelber Zonen auf, die sich sowohl in polaren als auch in unpolaren Bereichen befanden. Beim Besprühen mit Anisaldehyd- und Ehrlichs Reagenz sowie nach Zugabe von $2 \mathrm{~N} \mathrm{NaOH}$ wurde keine Farbänderung beobachtet. Letzteres deutete darauf hin, dass es sich wahrscheinlich nicht um Chinon-Derivate mit chelierten Hydroxygruppen handelte.

\subsubsection{Bestimmung der Taxonomie}

Der Stamm T436 wurde phänotypisch sowie genotypisch charakterisiert. Es zeigte sich, dass die 16S rRNA-Sequenz zu 98 \% jener von Salegentibacter holothuriorum homolog ist.

Der Stamm bildete auf Agar-Platten beige gefärbte Kolonien aus. Optimales Wachstum wurde bei Temperaturen zwischen 20 und $25{ }^{\circ} \mathrm{C}$ erreicht, wobei $30^{\circ} \mathrm{C}$ nicht überschritten werden durften. Es handelt sich somit um einen psychrotoleranten Stamm, der Gramnegativ, Oxidase- sowie Katalase-positiv ist und Nitrat zu reduzieren vermag. Gelatine und Äsculin können abgebaut werden, Schwefelwasserstoff wird im Stoffwechsel nicht gebildet. T436 zeigt weder $\beta$-Galaktosidase- noch Arginin-, Lysin- sowie Ornithin-Decarboxylaseaktivität. Im SFN-Biolog Verwertungstest können im Gegensatz zu Zuckerverbindungen fettähnliche Substanzen wie Tween 40 und Tween 80 metabolisiert werden.

\subsubsection{4-Nitrophenol}

Der hellgelbe Feststoff 40 fiel im Dünnschichtchromatogramm $\left(R_{f}=0.60, \mathrm{CH}_{2} \mathrm{Cl}_{2} / \mathrm{MeOH}\right.$ 9:1) durch UV-Löschung bei $254 \mathrm{~nm}$ auf.

Die Verbindung zeigte im APCI-Massenspektrum ein Ion bei $m / z=138[\mathrm{M}-\mathrm{H}]^{-}$. Im ${ }^{1} \mathrm{H}-$ NMR-Spektrum waren lediglich charakteristische Signale für zwei benachbarte aromatische Protonen bei $\delta=8.12$ und 6.88 detektierbar. Unter Einbeziehung der aus der Molmasse abgeleiteten Summenformel musste es sich daher um ein symmetrisches Molekül handeln. Im IR-Spektrum waren typische Banden für eine Nitrogruppe bei 1594 und $1339 \mathrm{~cm}^{-1}$ vorhanden. Dies legte die Vermutung nahe, dass ein hydroxy- sowie nitrosubs- 
tituierter Aromat vorlag, was durch die für 4-Nitrophenol (40) charakteristische Absorption bei $309 \mathrm{~nm}$ im UV-Spektrum bestätigt wurde.<smiles>O=[N+]([O-])c1ccc(O)cc1</smiles>

Verbindung 40 ist bereits als Stoffwechselprodukt von Stephanaspora caroticolor isoliert worden. ${ }^{70}$ Dieser auch unter dem Namen Karottentrüffel bekannte seltene Gasteromycet ist in Laub- und Mischwäldern beheimatet und bevorzugt lehmige, kalkhaltige Böden. Die etwa 1 bis $3 \mathrm{~cm}$ großen knollenförmigen Fruchtkörper wachsen hypogäisch und sind an ihrer orangegelben Farbe zu erkennen.

\subsubsection{4-Hydroxy-3-nitro-phenyl-Derivate}

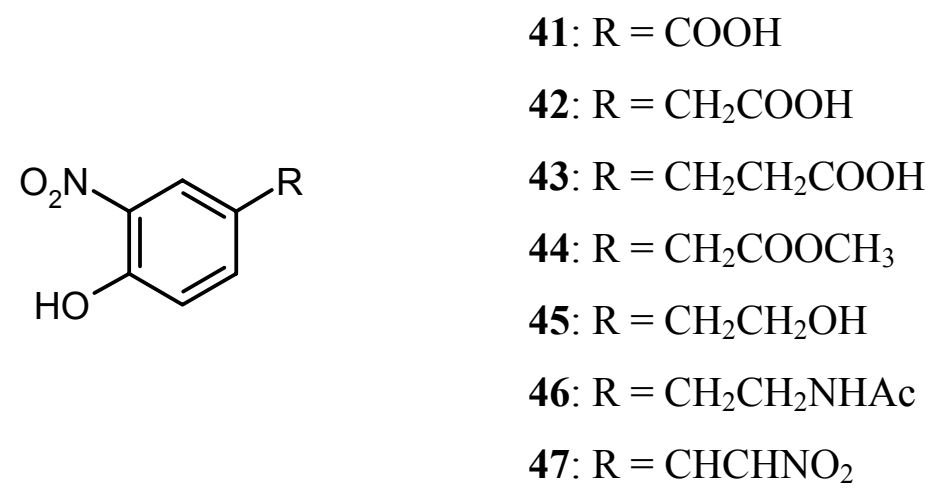

\section{4-Hydroxy-3-nitrobenzoesäure}

Verbindung 41 wurde in einer Ausbeute von $0.09 \mathrm{mg} / \mathrm{l}$ als gelber Feststoff isoliert. Die Substanz zeichnete sich durch relativ hohe Polarität aus, was sich im $R_{f}$-Wert von 0.25 bei Verwendung des Laufmittelgemischs $\mathrm{CH}_{2} \mathrm{Cl}_{2} / \mathrm{MeOH}$ 9:1 widerspiegelte.

Im EI-Massenspektrum zeigte 41 das Ion höchster Masse bei $m / z=183$. Im ${ }^{1} \mathrm{H}-\mathrm{NMR}$ Spektrum fanden sich für einen 1,2,4-trisubstituierten Aromaten charakteristische Signale bei $\delta=8.66,8.15$ und 7.13. Das ${ }^{13} \mathrm{C}-\mathrm{NMR}$-Spektrum wies eine auffällige Resonanz bei $\delta=169.8$ auf, die einem Carboxyl-Kohlenstoffatom zugeschrieben wurde. Im UV-Spek- 
trum war ein Absorptionsmaximum bei $340 \mathrm{~nm}$ vorhanden. Aus dem IR-Spektrum konnten Banden für das aromatische Grundgerüst bei 1626 und $1574 \mathrm{~cm}^{-1}$, für eine Nitrogruppe bei 1337 sowie $1542 \mathrm{~cm}^{-1}$ und für eine Arylcarbonsäure-Einheit bei $1685 \mathrm{~cm}^{-1}$ entnommen werden. In Kombination mit den aus einem HMBC-Spektrum erhaltenen Informationen konnte 41 als 4-Hydroxy-3-nitrobenzoesäure (41) identifiziert werden.

4-Hydroxy-3-nitrobenzoesäure (41) war bisher nur als Syntheseprodukt bekannt.

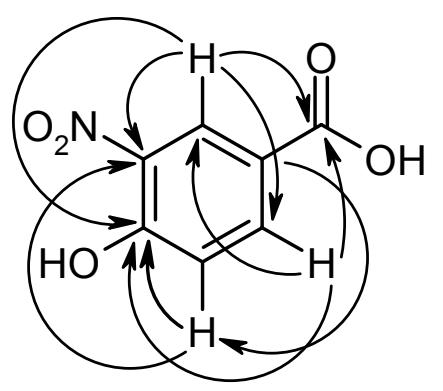

\begin{abstract}
Abbildung 32 Aus HMBC-Korrelationen abgeleitete Struktur von 4-Hydroxy-3-nitrobenzoesäure (41).
\end{abstract}

\title{
4-Hydroxy-3-nitrophenyl-essigsäure
}

Verbindung 42, die in einer Ausbeute von $0.15 \mathrm{mg} / \mathrm{l}$ als gelber Feststoff isoliert wurde, war im Dünnschichtchromatogramm $\left(R_{f}=0.39, \mathrm{CH}_{2} \mathrm{Cl}_{2} / \mathrm{MeOH} 9: 1\right)$ durch ihre Eigenfarbe und UV-Löschung bei $254 \mathrm{~nm}$ aufgefallen.

Im ${ }^{1} \mathrm{H}-\mathrm{NMR}-$ Spektrum zeigte $\mathbf{4 2}$ hinsichtlich des Kopplungsmusters im Aromatenbereich deutliche Ähnlichkeit zu 41. Außer einem geringen Unterschied in der chemischen Verschiebung der aromatischen Protonen war im ${ }^{1} \mathrm{H}-\mathrm{NMR}-S p e k t r u m$ von 42 im Gegensatz zu 41 ein zusätzliches Singulett bei $\delta=3.63$ vorhanden, das einer Methylengruppe zugeordnet wurde. Mittels EI-Massenspektrometrie wurde die Masse $m / z=197$ bestimmt, was einer Differenz von vierzehn im Vergleich zu Verbindung 41 entsprach. Im UV-Spektrum war ein Absorptionsmaximum bei $354 \mathrm{~nm}$ vorhanden. Das IR-Spektrum von 42 zeigte im Vergleich mit dem von 41 identische Banden für funktionelle Gruppen, jedoch lag die Absorption der Carboxylgruppe bei $1698 \mathrm{~cm}^{-1}$, was auf deren Verknüpfung mit einer gesättigten Alkylkette hindeutete. Diese Informationen führten in einer Datenbanksuche mit AntiBase ${ }^{30}$ zu 4-Hydroxy-3-nitrophenyl-essigsäure (42). Ein Vergleich der ${ }^{1} \mathrm{H}-\mathrm{NMR}$ Daten mit Literaturwerten bestätigte die Zuordnung. ${ }^{75}$ 


\section{4-Hydroxy-3-nitrophenyl-propionsäure}

Die Verbindung 43 war auf dem Dünnschichtchromatogramm hinsichtlich UV-Löschung und Anfärbeverhalten identisch mit 42, zeichnete sich aber durch eine niedrigere Polarität aus $\left(R_{f}=0.82, \mathrm{CH}_{2} \mathrm{Cl}_{2} / \mathrm{MeOH} 9: 1\right)$. Die Substanz konnte in einer Ausbeute von $0.08 \mathrm{mg} / 1$ als gelber Feststoff isoliert werden.

Das ${ }^{1}$ H-NMR-Spektrum von 43 stimmte im Aromatenbereich mit dem von 42 überein. Auffälligster Unterschied war das Fehlen der Methylengruppe bei $\delta=3.63$, an deren Stelle bei 43 die Signale für eine Ethylengruppe bei $\delta=2.93$ und 2.67 traten. Im EI-Massenspektrum zeigte 43 das Ion höchster Masse bei $m / z=211$. Dies entsprach einem im Vergleich zu 42 um vierzehn Einheiten höheren Molekulargewicht. Die UV- und IR-Spektren ergaben dieselben Absorptionsbanden wie bei Verbindung 42. Es musste sich folglich bei $\mathbf{4 3}$ um 4-Hydroxy-3-nitrophenyl-propionsäure (43) handeln. Ein Vergleich der spektroskopischen Daten mit Literaturwerten bestätigte die Zuordnung. ${ }^{71}$

4-Hydroxy-3-nitrophenyl-essigsäure (42) und 4-Hydroxy-3-nitrophenyl-propionsäure (43) wurden erstmals aus Streptomyces hygroscopicus isoliert und zeigen antibiotische Aktivität gegen Clostridium difficile. ${ }^{71}$ 4-Hydroxy-3-nitrophenyl-essigsäure (42) ist darüber hinaus phytotoxisch $^{72}$, verursacht eine 600proz. Erhöhung der Ammoniakfreisetzung bei Kiefersamen $^{73}$ und hemmt die Synthese von Chlorophyll bei Klee und Wasserlinse. ${ }^{74}$

\section{4-Hydroxy-3-nitrophenyl-essigsäuremethylester}

Substanz 44 wurde als bei $254 \mathrm{~nm}$ UV-löschender gelber Feststoff in einer Ausbeute von $0.09 \mathrm{mg} / 1$ erhalten, dessen Polarität zwischen der von 42 und 43 lag $\left(R_{f}=0.76, \mathrm{CH}_{2} \mathrm{Cl}_{2} /\right.$ $\mathrm{MeOH}$ 9:1).

Im ${ }^{1}$ H-NMR-Spektrum zeigte sich im Gegensatz zu demjenigen von Verbindung 42 eine zusätzliche Methoxygruppe bei $\delta=3.69$. Im EI-Massenspektrum war ein Ion höchster Masse bei $m / z=211 \mathrm{zu}$ erkennen, was einer Differenz von vierzehn Einheiten im Vergleich mit 42 entsprach. Im IR-Spektrum waren Banden für Phenyl- und Nitrogruppen sowie für eine Esterfunktion bei $1737 \mathrm{~cm}^{-1}$ sichtbar. Das UV-Spektrum war identisch mit dem von 42 und 43. Aus der Kombination dieser Informationen wurde Verbindung 44 als 4-Hydroxy-3-nitrophenyl-essigsäuremethylester (44) identifiziert. Ein Vergleich der NMRDaten mit Literaturwerten bestätigte die Zuordnung. ${ }^{75}$ 


\section{2-(4-Hydroxy-3-nitrophenyl)ethanol}

Die Substanz 45 wurde in einer Ausbeute von 0.13 mg/l aus einem 20 l-Fermentationsansatz von Stamm T436 isoliert. Die Verbindung fiel im Dünnschichtchromatogramm $\left(R_{f}=0.59, \mathrm{CH}_{2} \mathrm{Cl}_{2} / \mathrm{MeOH} 9: 1\right)$ als gelbe Zone auf, die UV-Licht bei $254 \mathrm{~nm}$ löschte.

UV- und ${ }^{1}$ H-NMR-Spektren von 45 zeigten wiederum fast vollständige Übereinstimmung mit denjenigen von 43. Beide ${ }^{1} \mathrm{H}-\mathrm{NMR}-\mathrm{Spektren}$ besaßen identische Signalaufspaltungen, jedoch war die chemische Verschiebung der Tripletts bei $\delta=2.93$ und 2.67 auf 3.86 und 2.84 verschoben. Im IR-Spektrum war eine zusätzliche Bande für eine Hydroxygruppe vorhanden, während die der Carboxyl-Funktion fehlte. Im EI-Massenspektrum zeigte 45 das Ion höchster Masse bei $m / z=183$. Die Substanz wurde als 2-(4-Hydroxy-3-nitrophenyl)ethanol (45) identifiziert. Ein Vergleich der NMR-Daten mit publizierten Werten bestätigte die Zuordnung. ${ }^{75}$

\section{$N$-(4-Hydroxy-3-nitrophenylethyl)acetamid}

Substanz 46 zeichnete sich durch UV-Löschung bei $254 \mathrm{~nm}$ und fehlende Farbreaktion beim Besprühen mit Anisaldehyd aus. Die Verbindung konnte als gelber Feststoff in einer Ausbeute von $0.4 \mathrm{mg} / \mathrm{l}$ aus dem Kulturfiltrat des Stammes T436 isoliert werden.

Das ${ }^{1}$ H-NMR-Spektrum von 46 zeigte im Gegensatz zu denjenigen von 43 und 45 ein zusätzliches Singulett bei $\delta=1.93$, welches einer Acetylgruppe zugeschrieben wurde. Im EI-Massenspektrum lag das Ion höchster Masse bei $m / z=224$. Das IR-Spektrum wies außer Hydroxy-, Phenyl-, und Nitro-Banden auch solche von sekundären Amin- sowie Amidgruppen bei $3300 \mathrm{~cm}^{-1}$ bzw. 1634 und $1532 \mathrm{~cm}^{-1}$ auf. Eine Suche in AntiBase ${ }^{30}$ und ein Vergleich der spektroskopischen Daten mit Literaturwerten ${ }^{75}$ führten zu $N$-(4-Hydroxy3-nitrophenylethyl)acetamid (46).

2-(4-Hydroxy-3-nitrophenyl)ethanol (45) und $N$-(4-Hydroxy-3-nitrophenylethyl)acetamid (46) wurden erstmals von SVIRIDOV et al. aus Pyricularia oryzae Cav. isoliert. ${ }^{75}$ Dieser Deuteromycet ist der Erreger von Pyriculariosis, einer bei Reispflanzen verbreiteten Erkrankung. 2-(4-Hydroxy-3-nitrophenyl)ethanol (45) zeigte beim Test eine wachstumshemmende Aktivität bei Pflanzen. 


\section{2-Nitro-4-(2'-nitroethenyl)phenol}

Auf dem Dünnschichtchromatogramm $\left(R_{f}=0.83, \mathrm{CH}_{2} \mathrm{Cl}_{2} / \mathrm{MeOH} 9: 1\right)$ fiel 47 durch gelbe UV-Löschung bei $254 \mathrm{~nm}$ auf, jedoch wies keine Anfärbung beim Besprühen mit Anisaldehyd auf. Die Verbindung wurde als orangefarbener Feststoff in einer Ausbeute von $0.03 \mathrm{mg} / \mathrm{l}$ isoliert.

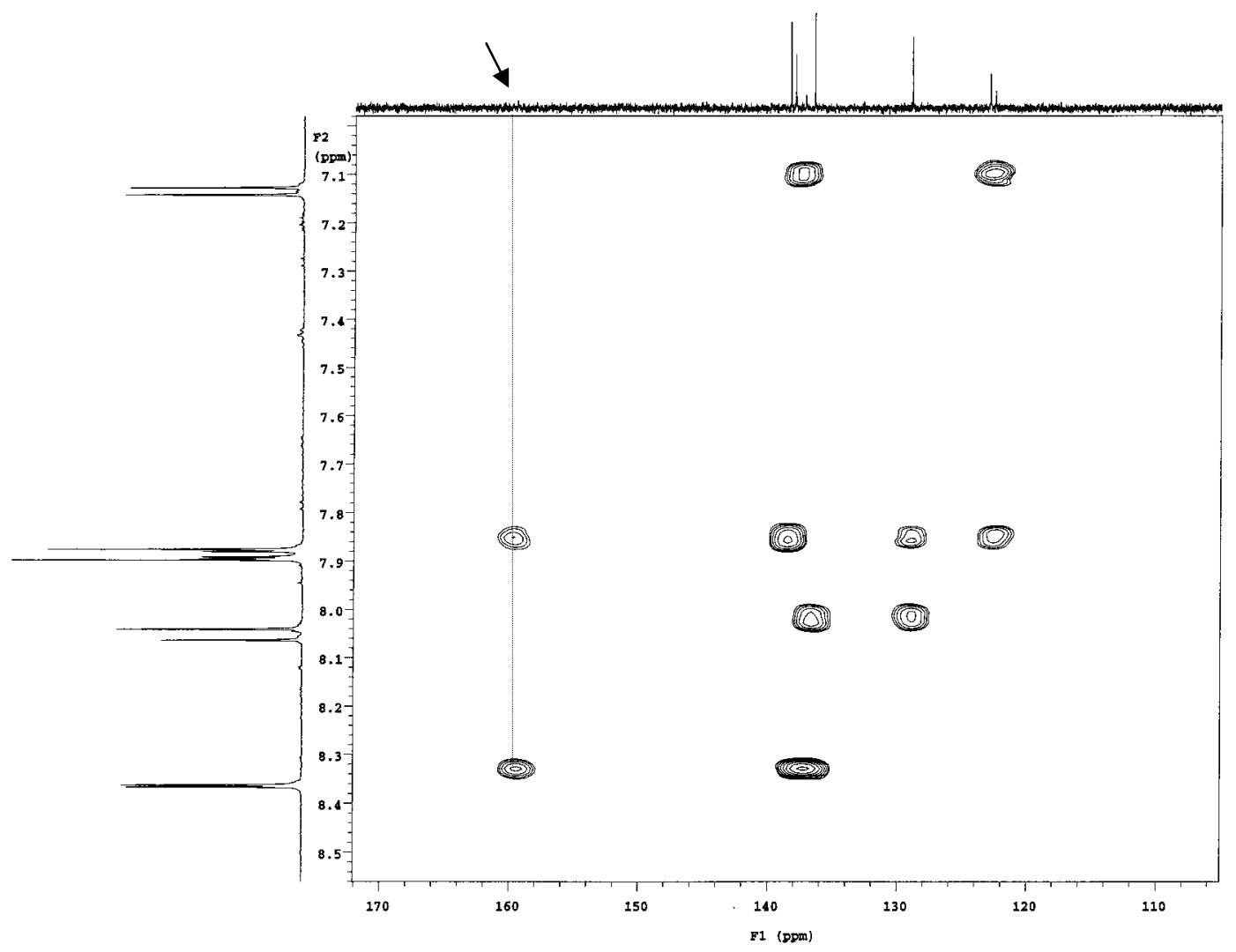

Abbildung 33 Das HMBC-Spektrum $\left(\mathrm{CD}_{3} \mathrm{OD}, 600 \mathrm{MHz}\right)$ von 2-Nitro-4-(2'-nitroethenyl)-phenol (47).

Im ${ }^{1}$ H-NMR-Spektrum waren drei Signale für aromatische Methingruppen bei $\delta=8.36$, 7.87 und 7.10 sichtbar, die anhand ihres Kopplungsmusters einem 1,2,4-trisubstituierten Aromaten zugeordnet werden konnte. Zusätzlich waren zwei Dubletts bei $\delta=8.05$ und 7.87 detektierbar, die aufgrund ihrer chemischen Verschiebung und der zugehörigen Kopplungskonstante $\left({ }^{3} J=13.5 \mathrm{~Hz}\right)$ zu einer trans-ständigen Doppelbindung gehören mussten. Das EI-Massenspektrum zeigte ein Ion höchster Masse bei $m / z=210$, aus dem durch Hochauflösung die Summenformel $\mathrm{C}_{8} \mathrm{H}_{6} \mathrm{~N}_{2} \mathrm{O}_{5}$ ermittelt wurde. Im ${ }^{13} \mathrm{C}$-NMR-Spektrum waren sieben Signale sichtbar. Erst im HMBC-Experiment konnte die fehlende achte Resonanz detektiert und die Strukturfragmente verknüpft werden. Dem UV-Spektrum 
waren Absorptionen bei 318, 333 und $435 \mathrm{~nm}$ zu entnehmen. Im IR-Spektrum waren eine Alkenbande bei $3110 \mathrm{~cm}^{-1}$, eine Arylalkenbande bei $1624 \mathrm{~cm}^{-1}$ sowie eine Nitrobande bei 1537 und $1342 \mathrm{~cm}^{-1}$ sichtbar. Die Verknüpfung der erhaltenen spektroskopischen Daten führte zu dem Ergebnis, dass 2-Nitro-4-(2'-nitroethenyl)phenol (47) vorlag.

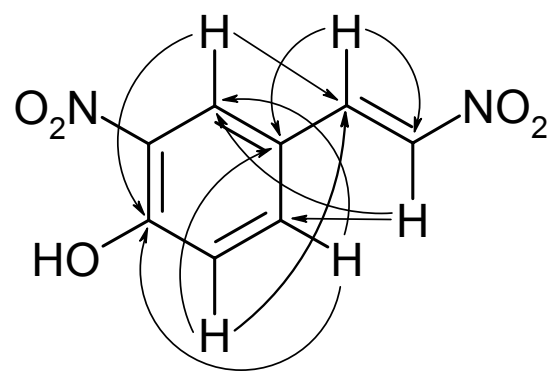

Abbildung 34 Aus HMBC-Korrelationen abgeleitete Struktur von 47

2-Nitro-4-(2'-nitroethenyl)phenol (47) wurde erstmals im Jahr 1992 aus Blättern der indischen Mangrove Sonneratia acida isoliert. $^{76}$

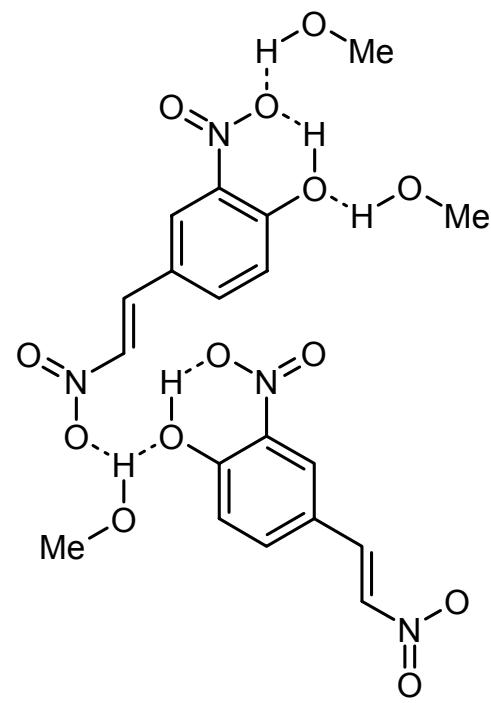

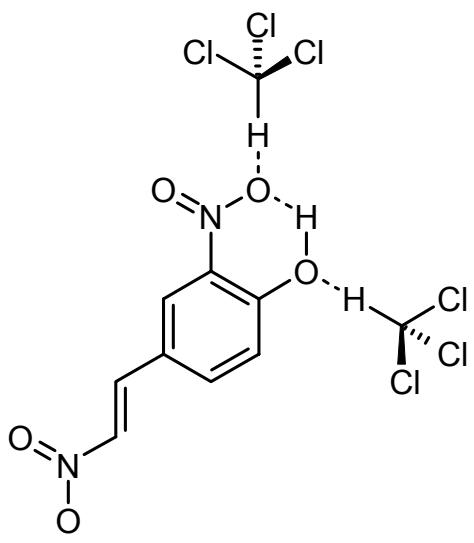

Abbildung 35 Schematische Darstellung der Wasserstoffbrückenbindungen zwischen den Molekülen der Lösungsmittel und den von 2-Nitro-4-(2'-nitroethenyl)phenol (47).

Bei 47 handelt es sich um ein annähernd planares Molekül, in dem ein Sauerstoffatom der ortho-ständigen Nitrogruppe eine intramolekulare Wasserstoffbrückenbindung mit der benachbarten Hydroxygruppe eingeht. Die Möglichkeit zur Ausformung zweier polymorpher Zustände liegt darin begründet, dass es beim Kristallisationsprozess zu einem Konkurrenzeffekt zwischen der Ausbildung von intra- und intermolekularen Wasserstoff- 
brückenbindungen kommt. So binden die aziden Protonen von Methanol an die Sauerstoffatome der Phenol- sowie der Nitrogruppe. Infolgedessen wird beim Entfernen des Lösungsmittels die Ausbildung intermolekularer Wasserstoffbrückenbindungen begünstigt, was zur gelben polymorphen Form führt. Das als schwächerer Protonendonator fungierende Chloroform hingegen bedingt die Ausformung intramolekularer Wasserstoffbrücken, was zu einem eingeschränkten gegenseitigen Kontakt der Moleküle führt und so die bevorzugte Bildung orangefarbener Kristalle hervorruft. Die letztgenannte polymorphe Form ist weniger stabil. $^{77}$

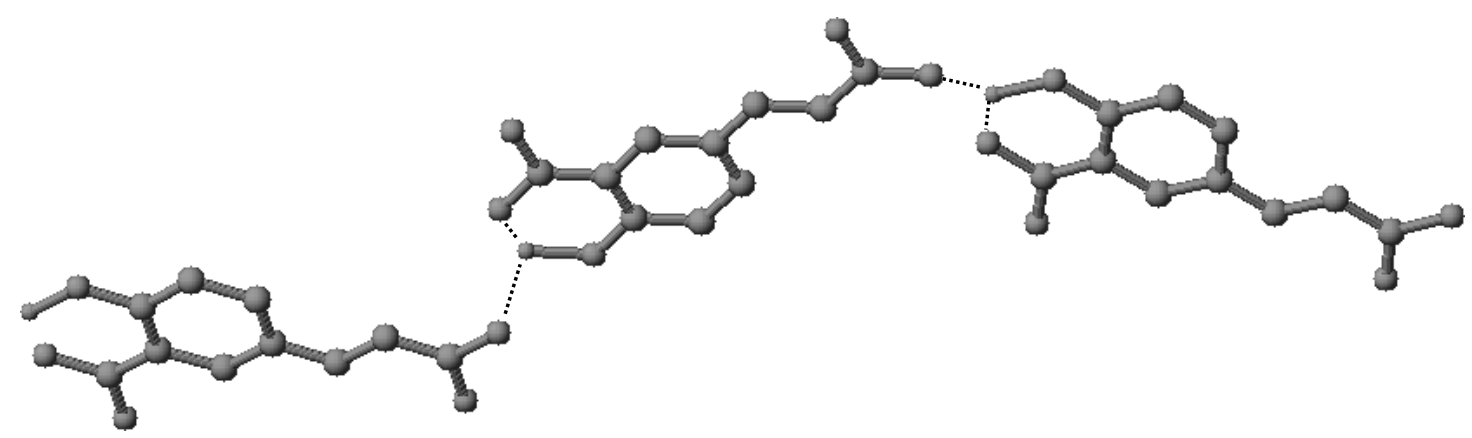

Abbildung 36 Dreidimensionale Darstellung der gelben polymorphen Form von 2-Nitro4-(2'-nitroethenyl)phenol (47).

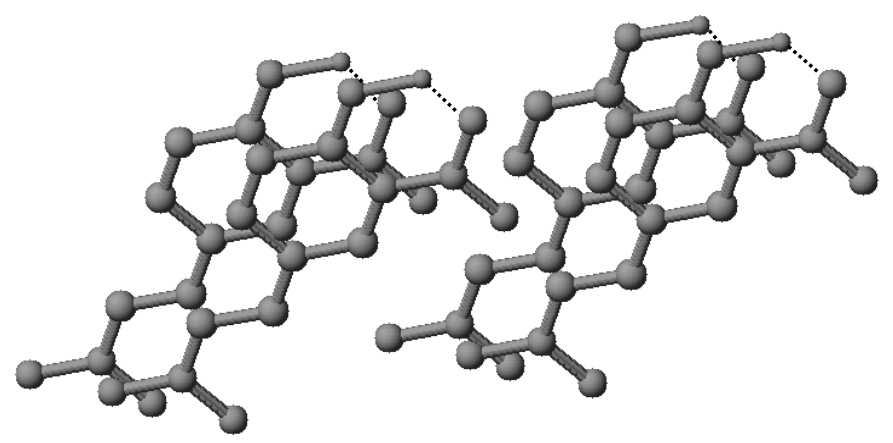

Abbildung 37 Dreidimensionale Darstellung der orangefarbenen polymorphen Form von 2-Nitro-4-(2'-nitroethenyl)phenol (47). 


\subsubsection{3,5-Dinitro-4-hydroxy-phenyl-Derivate}<smiles>[R]c1cc([N+](=O)[O-])c(O)c([N+](=O)[O-])c1</smiles>

48: $\mathrm{R}=\mathrm{CH}_{2} \mathrm{COOCH}_{3}$

49: $\mathrm{R}=\mathrm{CH}_{2} \mathrm{CH}_{2} \mathrm{COOH}$

50: $\mathrm{R}=\mathrm{CH}_{2} \mathrm{CH}_{2} \mathrm{Cl}$

51: $\mathrm{R}=\mathrm{CH}_{2} \mathrm{CHClCOOCH}_{3}$

52: $\mathrm{R}=\mathrm{CH}_{2} \mathrm{CH}_{2} \mathrm{NHCOCH}_{3}$

53: $\mathrm{R}=\mathrm{CHCHNO}_{2}$

\section{3,5-Dinitro-4-hydroxy-phenyl-essigsäuremethylester}

Der orangefarbene Feststoff 48 fiel im Dünnschichtchromatogramm $\left(R_{f}=0.36, \mathrm{CH}_{2} \mathrm{Cl}_{2} /\right.$ $\mathrm{MeOH}$ 9:1) durch die intensive Eigenfarbe sowie gelbe Fluoreszenz bei $254 \mathrm{~nm}$ auf. Verbindung 48 konnte in einer Ausbeute von $0.02 \mathrm{mg} / \mathrm{l}$ isoliert werden.

Im ${ }^{1}$ H-NMR-Spektrum waren drei Singulett-Signale bei $\delta=7.96,3.70$ und 3.60 sichtbar. Ersteres konnte zwei symmetrischen aromatischen Protonen zugeordnet werden, während die beiden letztgenannten zu jeweils einer Methyl- bzw. einer Methylengruppe gehörten. Verbindung 48 wies im ESI-Massenspektrum ein Ion bei $m / z=255[\mathrm{M}-\mathrm{H}]^{-}$auf, aus dem per Hochauflösung die Summenformel $\mathrm{C}_{9} \mathrm{H}_{8} \mathrm{~N}_{2} \mathrm{O}_{7}$ bestimmt werden konnte. Diese ergab in einer Datenbanksuche mit AntiBase ${ }^{30}$ in Kombination mit den aus den ${ }^{1} \mathrm{H}-\mathrm{NMR}-\mathrm{Daten}$ erhaltenen Strukturfragmenten keinen Treffer. Die geringe Substanzmenge sowie die ohnehin problematische Nachweisbarkeit von ${ }^{13} \mathrm{C}$-Resonanzen quartärer Kohlenstoffatome, welche mit Nitrogruppen funktionalisiert sind, führte dazu, dass die Gesamtzahl an Kohlenstoffatomen von 48 nur in einem HMBC-Experiment detektiert werden konnte. Hier waren sieben Resonanzen erkennbar, von denen eine einer Ester- bzw. Amidgruppe, vier aromatischen Kohlenstoffatomen sowie zwei einer aliphatischen Kette zugeordnet wurden. Die vier aromatischen Signale konnten aus Symmetriegründen einem 1,2,4,6-tetrasubstituierten Phenylring zugeschrieben werden. Im UV-Spektrum war die für substituierte Dinitrophenolsysteme charakteristische Absorption bei $432 \mathrm{~nm}$ zu erkennen. Dem IR-Spektrum konnten Banden für Phenyl- bzw. Nitrogruppen sowie für eine Esterfunktion entnommen werden. Diese Informationen führten dazu, dass 48 als 3,5-Dinitro-4-hydroxyphenyl-essigsäuremethylester (48) identifiziert wurde. In der Literatur ist $\mathbf{4 8}$ bislang nicht als Naturstoff bekannt. 


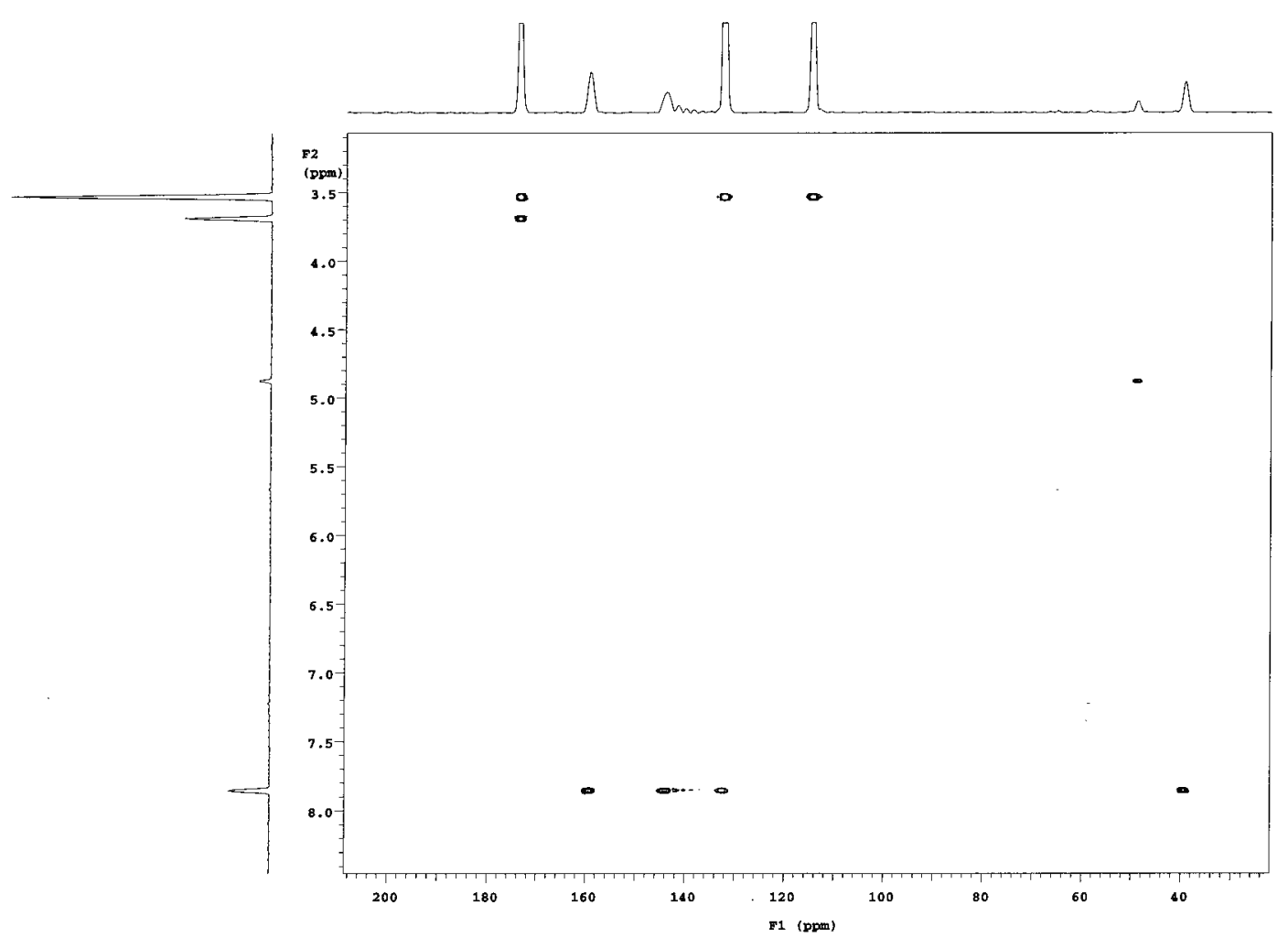

Abbildung 38 HMBC-Spektrum (600 MHz, $\mathrm{CD}_{3} \mathrm{OD}$ ) von 3,5-Dinitro-4-hydroxy-phenylessigsäuremethylester (48).

\section{3,5-Dinitro-4-hydroxy-phenyl-propionsäure}

Im Dünnschichtchromatogramm war 49 hinsichtlich UV-Löschung und Anfärbeverhalten identisch mit 48, zeichnete sich aber durch eine höhere Polarität aus $\left(R_{f}=0.16, \mathrm{CH}_{2} \mathrm{Cl}_{2} /\right.$ $\mathrm{MeOH}$ 9:1). Die Substanz 49 konnte in einer Ausbeute von $0.05 \mathrm{mg} / 1$ isoliert werden.

Das ${ }^{1} \mathrm{H}-\mathrm{NMR}-$ Spektrum des orangefarbenen Feststoffs stimmte im Aromatenbereich mit dem von 48 überein. Es fehlten jedoch die Singuletts der aliphatischen Protonen, an deren Stelle Signale für eine Ethylengruppe bei $\delta=2.89$ und 2.62 traten. Das ESI-Massenspektrum und die per Hochauflösung bestimmte Summenformel zeigten, dass es sich bei $\mathbf{4 8}$ und 49 um Isomere handelte. Die UV-Spektren beider Verbindungen waren identisch, ebenso konnten im IR-Spektrum Banden für Aromaten- und Nitrogruppen detektiert werden. Hier fehlte jedoch die Absorption der Estergruppe, stattdessen trat eine zusätzliche Bande für eine Säuregruppe bei $1712 \mathrm{~cm}^{-1}$ auf. Ausgehend von diesen Informationen wurde vermutet, dass es sich bei 49 um 3,5-Dinitro-4-hydroxyphenyl-propionsäure (49) handelte. Die im HMBC-Spektrum beobachteten Korrelationen bestätigten diese Vermutung. Verbindung 49 wurde bislang in der Literatur nicht als Naturstoff beschrieben. 


\section{3,5-Dinitro-4-hydroxy-phenyl-ethylchlorid}

Verbindung 50 zeichnete sich auf dem Dünnschichtchromatogramm durch dieselbe Eigenfarbe sowie identische UV-Eigenschaften, jedoch unterschiedliche Polarität $\left(R_{f}=0.44\right.$, $\mathrm{CH}_{2} \mathrm{Cl}_{2} / \mathrm{MeOH}$ 9:1) im Vergleich zu 48 und 49 aus. Der orangefarbene Feststoff $\mathbf{5 0}$ konnte in einer Ausbeute von $0.03 \mathrm{mg} / \mathrm{l}$ isoliert werden.

Das sich im ${ }^{1}$ H-NMR-Spektrum zeigende Signalmuster von $\mathbf{5 0}$ stimmte mit dem von 49 sehr gut überein. Jedoch erschienen die auftretenden Tripletts bei einer anderen chemischen Verschiebung. Im ESI-Massenspektrum fiel das Molekülion durch ein für Chlor typisches Isotopenverhältnis (3:1) auf. Auch die Hochauflösung bestätigte die Existenz dieses Elementes im Molekül. Das IR-Spektrum zeigte weder die Bande für eine Säurenoch für eine Estergruppierung. Es musste sich bei der Substanz folglich um 3,5-Dinitro-4hydroxyphenyl-ethylchlorid (50) handeln. Mit Hilfe des HMBC-Spektrums wurde die Struktur abgesichert. Verbindung $\mathbf{5 0}$ ist bisher in der Literatur weder als Naturstoff noch als Syntheseprodukt bekannt.

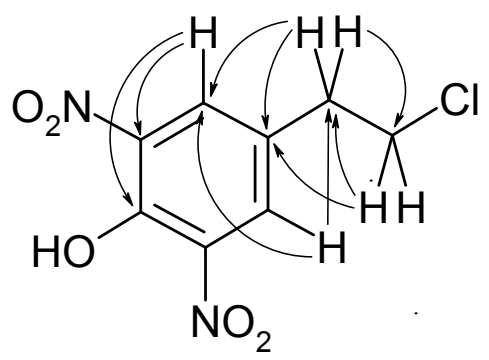

Abbildung 39 Aus HMBC-Korrelationen abgeleitete Strukturfragmente von 3,5-Dinitro4-hydroxy-phenyl-ethylchlorid (50).

\section{3,5-Dinitro-4-hydroxy-phenyl-2-chlor-propionsäuremethylester}

Die orangefarbene Substanz 51 wurde in einer sehr geringen Ausbeute von $0.04 \mathrm{mg} / \mathrm{l}$ isoliert und wies im Dünnschichtchromatogramm identisches UV- und Anfärbeverhalten wie die vorangehenden 3,5-Dinitro-4-hydroxyphenyl-Derivate auf.

Das ${ }^{1} \mathrm{H}-\mathrm{NMR}-$ Spektrum von 51 zeigte im aromatischen Bereich ebenfalls ein Singulett, das für zwei Protonen integrierte. Im aliphatischen Bereich befanden sich Signale für eine Methingruppe bei $\delta=4.56$, eine Methoxygruppe bei $\delta=3.73$ sowie für eine diastereotope Methylengruppe bei $\delta=3.18$ bzw. 3.06. Die chemische Verschiebung der Methingruppe legte die Vermutung nahe, dass diese mit einem Heteroatom sowie einer Carbonylfunktion 
verknüpft war. Im APCI-Massenspektrum trat ein Ion bei $m / z=303[\mathrm{M}-\mathrm{H}]^{-}$auf, das aufgrund seines Isotopenmusters auf das Vorhandensein eines Chloratoms im Molekül hinwies. Ebenso zeigte das IR-Spektrum eine starke Absorptionsbande bei $701 \mathrm{~cm}^{-1}$, welche charakteristisch für chlorsubstituierte Kohlenstoffatome ist. Ein Hochauflösung-ESI-Spektrum bestätigte die Summenformel von $\mathrm{C}_{10} \mathrm{H}_{9} \mathrm{ClN}_{2} \mathrm{O}_{7}$. Diese Ergebnisse führten zu dem Schluss, dass 3,5-Dinitro-4-hydroxy-phenyl-2-chlor-propionsäuremethylester (51) isoliert wurde. Aufgrund der geringen Substanzmengen konnten keine 2D-NMR-Experimente zur weiteren Absicherung der Struktur durchgeführt werden. Verbindung 51 war bisher weder als Naturstoff noch als Syntheseprodukt bekannt.

\section{$N$-(3,5-Dinitro-4-hydroxy-phenylethyl)acetamid}

Die ebenfalls als oranger Feststoff anfallende Substanz 52 war auf dem Dünnschichtchromatogramm hinsichtlich UV-Verhalten identisch mit den vorherigen Dinitrophenol-Verbindungen und konnte in einer Ausbeute von $0.14 \mathrm{mg} / 1$ isoliert werden.

Das ${ }^{1}$ H-NMR-Spektrum von 52 zeigte im Aromatenbereich große Ähnlichkeit zu dem von 50, während die aliphatischen Signale gut mit denen von Verbindung 46 übereinstimmten. Im ${ }^{13} \mathrm{C}-\mathrm{NMR}$-Spektrum waren acht Signale erkennbar, die aufgrund der Symmetrieeigenschaften des aromatischen Strukturelements zehn Kohlenstoffatomen zugeordnet wurden. Das ESI-Massenspektrum zeigte ein Ion bei $m / z=268$ [M-H]', zusätzlich zeichnete sich das IR-Spektrum von 52 durch große Ähnlichkeit zu dem von 46 aus. Die Kombination der beschriebenen Informationen ließen nur den Schluss zu, dass es sich bei 52 um N-(3,5Dinitro-4-hydroxy-phenylethyl)acetamid handelte. Die Struktur wurde durch die im HMBC-Spektrum auftretenden Korrelationen weiter abgesichert. Das DinitrophenolDerivat 52 war bislang ebenso weder als Naturstoff noch als Syntheseprodukt bekannt.

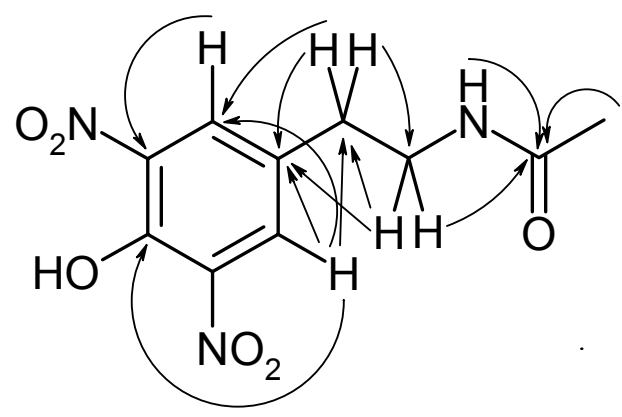

Abbildung 40 Aus HMBC-Korrelationen abgeleitete Strukturfragmente von $N-(3,5-$ Dinitro-4-hydroxy-phenylethyl)acetamid (52). 


\section{2,6-Dinitro-4-(2'-nitroethenyl)phenol}

Das ESI-Massenspektrum von 53 zeigte ein Ion bei $m / z=254$ [M-H]', was auf das Vorhandensein einer ungeraden Zahl an Stickstoffatomen im Molekül hinwies. Mittels Hochauflösung wurde die Summenformel $\mathrm{C}_{8} \mathrm{H}_{5} \mathrm{~N}_{3} \mathrm{O}_{7}$ bestimmt. Im ${ }^{1} \mathrm{H}-\mathrm{NMR}$-Spektrum waren ein Singulett bei $\delta=8.24$, das aufgrund der Integralhöhe zwei aromatischen Protonen zugeschrieben wurde, sowie zwei Dubletts bei $\delta=7.99$ und 7.76 zu erkennen. Letztere konnten aufgrund ihrer chemischen Verschiebung und der zugehörigen Kopplungskonstante $\left({ }^{3} J=13.5 \mathrm{~Hz}\right)$ einer trans-ständigen Doppelbindung zugeordnet werden. Die spektroskopischen Befunde führten im Vergleich mit den von Verbindung 47 erhaltenen zu dem Ergebnis, dass es sich bei 53 um 2,6-Dinitro-4-(2'-nitroethenyl)phenol (53) handeln musste. Aufgrund der geringen Substanzmengen konnten keine zusätzlichen 2D-NMRExperimente durchgeführt werden. Ebenfalls stellt 53 eine bislang nicht bekannte Verbindung dar.

\subsubsection{Pyriculamid}

Der gelbe Feststoff 55 fiel im Dünnschichtchromatogramm durch seine Eigenfarbe, UVLöschung bei $254 \mathrm{~nm}$. Die Substanz 55 wurde in einer Ausbeute von $0.3 \mathrm{mg} / 1$ aus dem Stamm T436 isoliert.

Das ${ }^{1}$ H-NMR-Spektrum von 55 zeigte im Aromatenbereich identische Signale wie die bereits beschriebenen 4-Hydroxy-3-nitrophenyl-Derivate. Im aliphatischen Bereich traten zahlreiche zusätzliche Resonanzen auf, die denen des Diketopiperazins Cyclo(tyrosylprolyl) (54) sehr ähnelten. Das ESI-Massenspektrum zeigte eine Molmasse von 305 an. Im ${ }^{13} \mathrm{C}$-NMR-Spektrum waren $14 \mathrm{C}$-Atome zu erkennen, von denen zwei $(\delta=168.2$ und 164.5) Ester- bzw. Amidgruppen zugeordnet werden konnten. Eine Suche in AntiBase ${ }^{30}$ mit den so erhaltenen Informationen führte zu Pyriculamid (55). Ein Vergleich der NMRDaten mit Literaturwerten bestätigte die Zuordnung. Das nitrierte Cyclo(tyrosylprolyl) 55 wurde erstmals aus Pyricularia oryzae isoliert. ${ }^{75}$<smiles></smiles> 


\title{
6.2.6 7-Hydroxy-3-(4-hydroxy-3-nitrophenyl)-4H-1-benzopyran-4-on (3'-Nitro- daidzein)
}

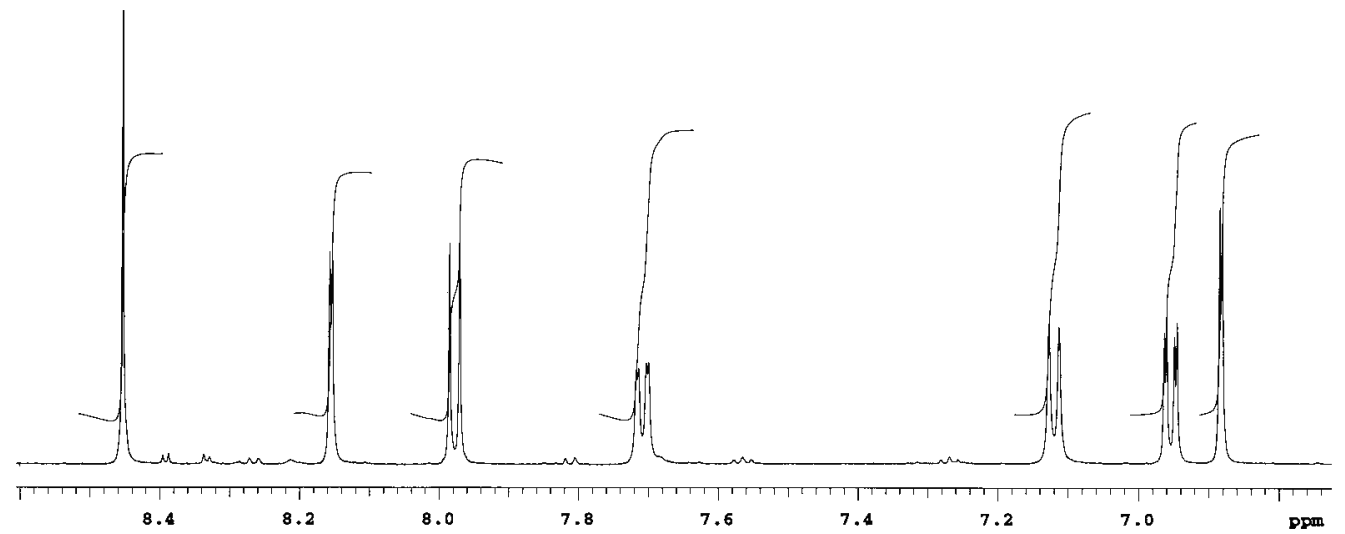

\begin{abstract}
Abbildung $41{ }^{1} \mathrm{H}-\mathrm{NMR}-\mathrm{Spektrum}\left(600 \mathrm{MHz},\left[\mathrm{D}_{6}\right] \mathrm{DMSO}\right)$ von 7-Hydroxy-3-(4-hydroxy3-nitrophenyl)-4H-1-benzopyran-4-on (56).
\end{abstract}

Die UV-löschende Verbindung 56 konnte in einer Ausbeute von 0.06 mg/l in Form eines gelben Feststoffs isoliert werden. Sie zeigte im ESI-Massenspektrum ein Ion bei $\mathrm{m} / \mathrm{z}=298$ $[\mathrm{M}-\mathrm{H}]^{-}$. Im ${ }^{1} \mathrm{H}-\mathrm{NMR}-$ Spektrum waren sieben aromatische Protonen bei $\delta=8.45,8.15$, 7.97, 7.71, 7.12, 6.94 und $6.88 \mathrm{zu}$ erkennen, von denen die letzten sechs anhand des Aufspaltungsmuster sowie der Kopplungskonstante zwei 1,2,4-trisubstituierten aromatischen Systemen zugeordnet werden konnten. Dem ${ }^{13} \mathrm{C}-\mathrm{NMR}-$ Spektrum konnte das Vorliegen von fünfzehn Kohlenstoffatomen entnommen werden, unter denen sich eine Carbonylgruppe $(\delta=174.3)$ befand. Durch hochauflösende Massenspektrometrie konnte die Summenformel $\mathrm{zu} \mathrm{C}_{15} \mathrm{H}_{9} \mathrm{NO}_{6}$ bestimmt werden. Anhand von 2D-NMR-Experimenten konnte ein Strukturvorschlag für 56 abgeleitet werden, demzufolge es sich um 7-Hydroxy3-(4-hydroxy-3-nitrophenyl)-4H-1-benzopyran-4-on (56) handeln musste. Durch Vergleich der NMR-spektroskopischen Daten mit Literaturwerten konnte die Zuordnung bestätigt werden.

Verbindung 56 wurde bereits 1997 aus dem gentechnisch veränderten Stamm Streptomyces sp. $\mathrm{K}_{3}$ isoliert. $^{78}$ 


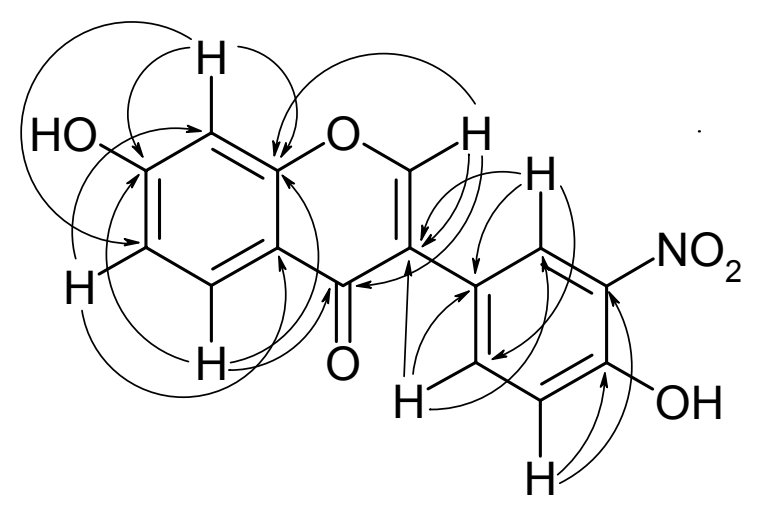

Abbildung 42 Aus HMBC-Korrelationen abgeleitete Strukturfragmente von 7-Hydroxy3-(4-hydroxy-3-nitrophenyl)-4H-1-benzopyran-4-on (56) .<smiles>O=c1c(-c2ccc(O)c([N+](=O)[O-])c2)coc2cc(O)ccc12</smiles>

56

\subsubsection{5,7-Dihydroxy-3-(3,5-dinitro-4-hydroxy-phenyl)-4H-1-benzopyran-4-on (3',5'-Dinitro-genistein)}

Der orangefarbene, bei $254 \mathrm{~nm}$ gelb fluoreszierende Feststoff 57 war in allen gängigen Lösungsmitteln mit Ausnahme von Dimethylsulfoxid schwer löslich.

Verbindung 57 wies im APCI-Massenspektrun ein Ion bei $m / z=359[\mathrm{M}-\mathrm{H}]^{-}$auf, aus dem mittels Hochauflösung die Summenformel $\mathrm{C}_{15} \mathrm{H}_{8} \mathrm{~N}_{2} \mathrm{O}_{9}$ erhalten wurde. Im ${ }^{1} \mathrm{H}-\mathrm{NMR}-\mathrm{Spek}$ trum waren Signale für eine $\mathrm{OH}-\mathrm{bzw}$. NH-Gruppe bei $\delta=12.78$ sowie fünf Methingruppen bei $\delta=8.27,8.03(2 \mathrm{H}), 6.12$ und 6.00 erkennbar. Die beiden letztgenannten wurden aufgrund ihrer chemischen Verschiebung und der zugehörigen Kopplungskonstante metaständigen aromatischen Protonen zugeordnet. Das Singulett bei $\delta=8.03$ integrierte für zwei Protonen, was dafür sprach, dass ein symmetrisch substituierter Phenylring vorlag. Dementsprechend wurden im ${ }^{13} \mathrm{C}-\mathrm{NMR}-$ Spektrum, das aus insgesamt dreizehn Resonanzen bestand, zwei Signale mit doppelter Intensität beobachtet. Mit Hilfe von 2D-NMRSpektren konnten die abgeleiteten Strukturfragmente verknüpft und die Substanz als 5,7Dihydroxy-3-(3,5-dinitro-4-hydroxy-phenyl)-4H-1-benzopyran-4-on (57) identifiziert werden. 
Verbindung 57 wurde erstmals 1999 aus dem genetisch veränderten Amikacin-Produzenten Streptomyces sp. K3 isoliert. ${ }^{79}$

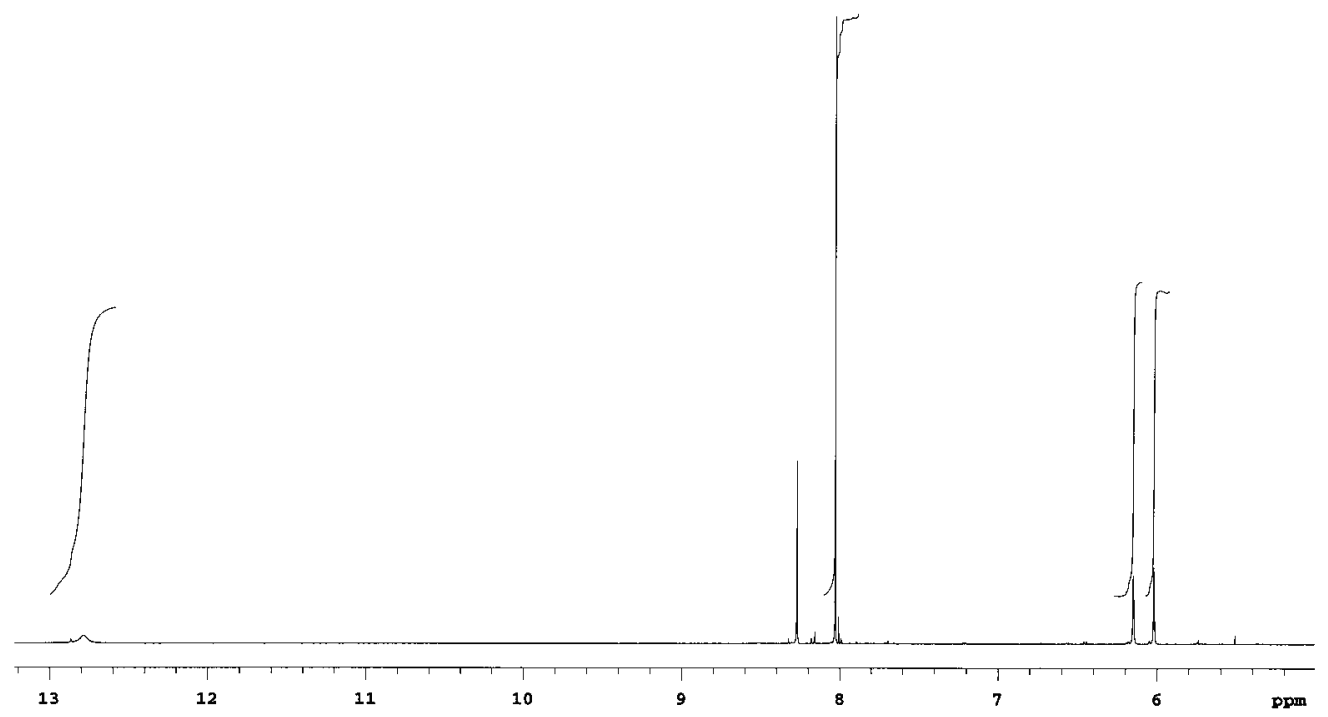

Abbildung $43{ }^{1}$ H-NMR-Spektrum (600 MHz, [D 6 ]DMSO) von 57.

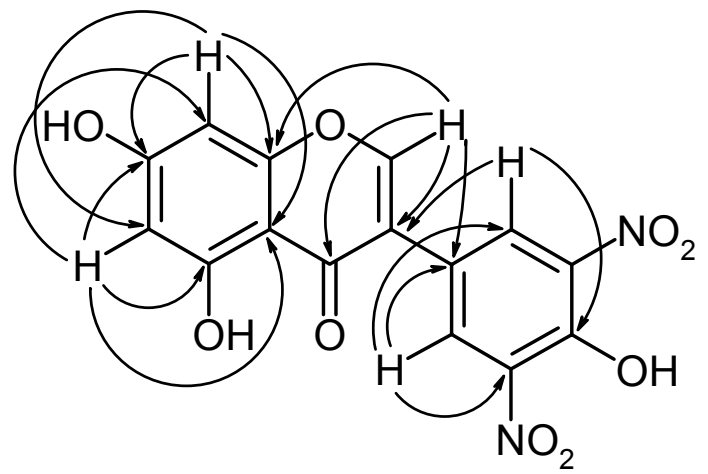

Abbildung 44 Aus HMBC-Korrelationen abgeleitete Strukturfragmente von 57.<smiles>O=c1c(-c2cc([N+](=O)[O-])c(O)c([N+](=O)[O-])c2)coc2cc(O)cc(O)c12</smiles> 


\subsubsection{Nitro- $N_{\beta}$-acetyltryptamine}

\section{2-Nitro- $N_{\beta}$-acetyltryptamin}

Die Substanz 58 fiel im Dünnschichtchromatogramm durch ihre gelbe Eigenfarbe und UVLöschung bei $254 \mathrm{~nm}$ auf, zeigte jedoch keine Farbreaktion beim Besprühen mit Anisaldehyd.

Im ESI-Massenspektrum war ein Ion bei $m / z=270[\mathrm{M}+\mathrm{Na}]^{+} \mathrm{zu}$ erkennen, welches auf eine ungerade Anzahl an Stickstoffatomen in der Verbindung hinwies. Eine HRESI-MSMessung führte zur Summenformel $\mathrm{C}_{12} \mathrm{H}_{13} \mathrm{~N}_{3} \mathrm{O}_{3}$. Anhand des IR-Spektrums konnte weiterhin auf die Anwesenheit einer Nitrogruppe geschlossen werden. Das ${ }^{1} \mathrm{H}-\mathrm{NMR}$ Spektrum zeigte im aromatischen Bereich Signale höherer Ordnung bei $\delta=7.74,7.41(2 \mathrm{H})$ und 7.18. Ferner konnte neben vier aliphatischen Protonen bei $\delta=3.51$ und 3.37, welche Methylengruppen zugeschrieben wurden, das Signal einer Acetylgruppierung bei $\delta=1.78$ detektiert werden. Im ${ }^{13} \mathrm{C}$-NMR-Spektrum waren zwölf Resonanzen erkennbar, unter denen sich die eines Carbonylkohlenstoffatoms bei $\delta=173.4$ befand. Somit war die Anwesenheit einer Ester- oder Amidgruppe im Molekül gesichert. Das komplexe Aufspaltungsmuster der aromatischen Protonen im ${ }^{1} \mathrm{H}-\mathrm{NMR}$-Spektrum erschwerte die Zuordnung der Position der Nitrogruppe. Die Strukturaufklärung gelang schließlich mit Hilfe eines HMBC-Korrelationsspektrums und führte $\mathrm{zu}$ 2-Nitro- $N_{\beta}$-acetyltryptamin (58). Das Indolderivat 58 war bisher weder als Naturstoff noch als Syntheseprodukt bekannt.

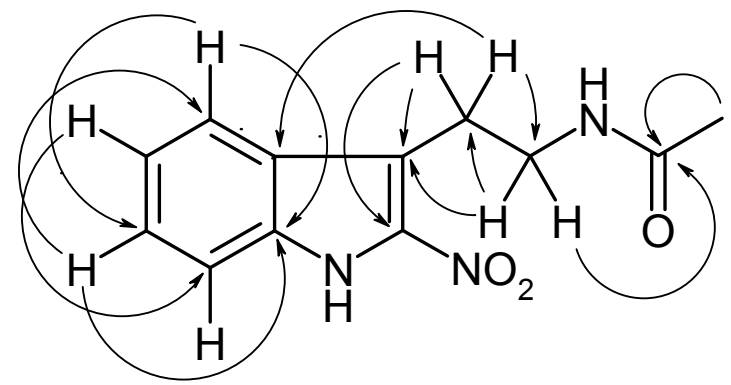

Abbildung 45 Aus HMBC-Korrelationen abgeleitete Strukturfragmente von 2-Nitro- $N_{\beta}$ acetyltryptamin (58).<smiles>CC(=O)NCCc1c([N+](=O)[O-])[nH]c2ccccc12</smiles> 


\section{6-Nitro- $N_{\beta}$-acetyltryptamin}

Der gelbe Feststoff 59 zeigte im Dünnschichtchromatogramm UV-Löschung bei $254 \mathrm{~nm}$. Im ESI-Massenspektrum war ein Ion bei $m / z=270[\mathrm{M}+\mathrm{Na}]^{+} \mathrm{zu}$ erkennen, was auf das Vorliegen einer zu 58 isomeren Verbindung hinwies. Das ${ }^{1} \mathrm{H}-\mathrm{NMR}-$ Spektrum zeigte im aromatischen Bereich Signale bei $\delta=8.31,7.92,7.68$ und 7.46, welche einem disubstituierten Indolgerüst zugeordnet werden konnten. Weiterhin waren Signale für zwei Methylengruppen bei $\delta=3.46$ und 2.97 sowie für eine Acetylgruppe bei $\delta=1.90$ erkennbar. Aus diesen Gründen wurde vermutet, dass es sich bei Verbindung 59 um ein $N_{B}$-Acetyltryptamin-Derivat handelte. Aufspaltungsmuster und chemische Verschiebungen im aromatischen Bereich deuteten auf eine Substitution in Position sechs des Indolgerüstes hin. Ein Vergleich der spektroskopischen Daten mit denjenigen von 58 führte zu 6-Nitro- $N_{\beta}$ acetyltryptamin (59). Diese Verbindung war bisher weder als Naturstoff noch als Syntheseprodukt bekannt.<smiles>CC(=O)NCCc1c[nH]c2cc([N+](=O)[O-])ccc12</smiles>

\section{7-Nitro- $N_{\beta}$-acetyltryptamin}

Der gelbe Feststoff 60 wies identisches Verhalten im Dünnschichtchromatogramm auf und zeigte im ESI-Massenspektrum dasselbe Quasimolekülion wie die Verbindungen $\mathbf{5 8}$ und 59. Im ${ }^{1}$ H-NMR-Spektrum waren neben aromatischen Signalen bei $\delta=7.78,7.71,7.38$ und 7.20 zusätzliche Resonanzen für zwei Methylengruppen bei $\delta=3.34$ und 3.04 sowie für eine Acetylgruppe bei $\delta=1.89$ erkennbar. Aufspaltungsmuster und chemische Verschiebungen der aromatischen Signale legten das Vorliegen eines an Position sieben substituierten Indolgerüstes nahe. Es musste sich bei $\mathbf{6 0}$ somit um in der Literatur bisher nicht beschriebenes 7-Nitro- $N_{\beta}$-acetyltryptamin (60) handeln.<smiles>CC(=O)NCCc1c[nH]c2c([N+](=O)[O-])cccc12</smiles> 


\subsubsection{Salegentipyrrol A}

Die sich mit Anisaldehyd in der Wärme orange anfärbende Verbindung 61 löschte UVLicht der Wellenlänge $254 \mathrm{~nm}$, war im Gegensatz zu den bisher aus diesem Stamm isolierten Verbindungen jedoch farblos. Die Substanz 61 konnte lediglich in einer geringen Ausbeute von $0.02 \mathrm{mg} / \mathrm{l}$ isoliert werden.

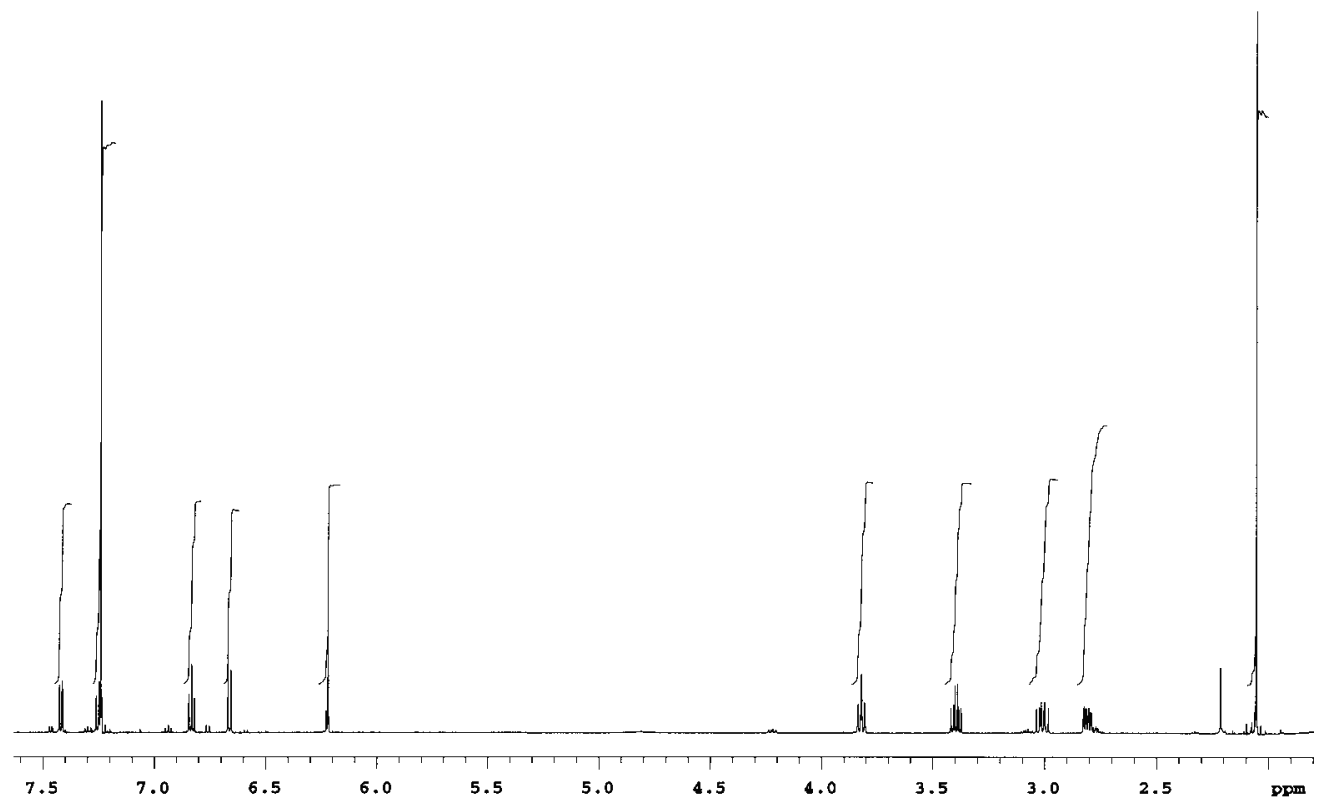

Abbildung $46{ }^{1} \mathrm{H}-\mathrm{NMR}-\mathrm{Spektrum}(600 \mathrm{MHz})$ von Salegentipyrrol A (61) in $\mathrm{CDCl}_{3}$.

Mit Hilfe des ${ }^{13} \mathrm{C}-\mathrm{NMR}-$ Spektrums und einer HRESI-MS-Messung wurde 61 die Summenformel $\mathrm{C}_{12} \mathrm{H}_{13} \mathrm{~N}_{3} \mathrm{O}_{3}(\mathrm{M}=247 \mathrm{~g} / \mathrm{mol})$ zugewiesen. Dem ${ }^{1} \mathrm{H}-\mathrm{NMR}-\mathrm{Spektrum}$ waren Signale für eine Acetylgruppe $(\delta=2.06)$, zwei diastereotope Methylengruppen $(\delta=3.84,3.40$, 3.01 und 2.81), eine Methingruppe $(\delta=6.23)$, vier benachbarte aromatische Protonen $\left(\delta=7.42,7.24,6.84\right.$ und 6.66) sowie ein durch $\mathrm{D}_{2} \mathrm{O}$ austauschbares Proton $(\delta=5.34) \mathrm{zu}$ entnehmen. Das ${ }^{13} \mathrm{C}$-NMR-Spektrum zeigte die Existenz einer Amid- oder Estergruppe $(\delta=170.1)$ an. Mit Hilfe eines ${ }^{1} \mathrm{H},{ }^{1} \mathrm{H}-\mathrm{COSY}$-Experimentes konnten die in Abbildung 47 gezeigten Strukturelemente abgeleitet werden. Mittels eines HMBC-Experiments gelang die Verknüpfung der erhaltenen Fragmente. 

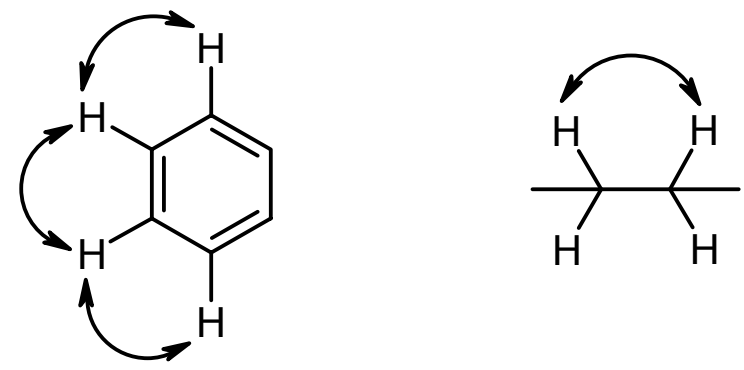

Abbildung $47{ }^{1} \mathrm{H},{ }^{1} \mathrm{H}-\mathrm{COSY}$-Fragmente von Salegentipyrrol A (61).

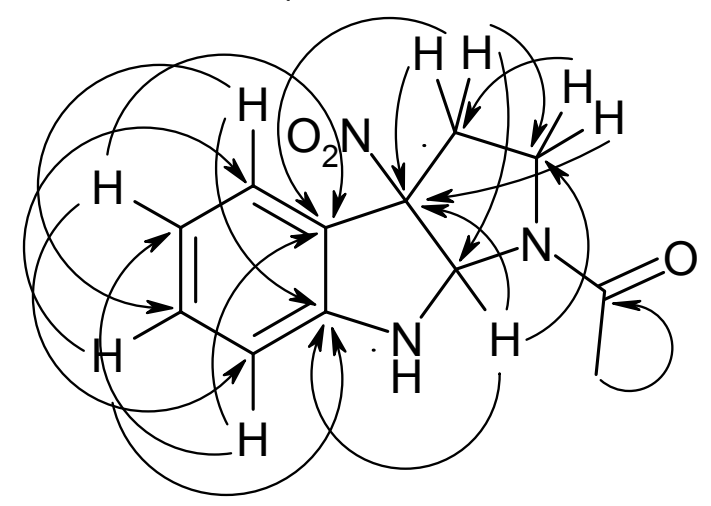

Abbildung 48 Aus HMBC-Korrelationen abgeleitete Strukturfragmente von Salegentipyrrol A (61).<smiles>CC(=O)N1CCC2([N+](=O)[O-])c3ccccc3NC12</smiles>

61<smiles>CC(=O)N1CCC2(O)c3ccccc3N[C@H]12</smiles>

62

Verbindung 61 wurde als 1-Acetyl-1,2,3,3a,8,8a-hexahydropyrrolo[2,3-b]-3a-nitro-indol (61) identifiziert und Salegentipyrrol A genannt. Die dieser bislang nicht beschriebenen Substanz strukturell nah verwandte Verbindung 62 wurde bereits aus einem Stamm der Spezies Streptomyces staurosporeus isoliert, der als Produzent des Proteinkinase-CHemmstoffes Staurosporin (63) bekannt ist. ${ }^{80}$ Die Produktion von 62 wurde durch Zufütterung von Tryptamin-hydrochlorid angeregt, ${ }^{81}$ woraus sich ein plausibler Biosyntheseweg für die Bildung von $\mathbf{6 1}$ ausgehend von $N_{\beta}$-Acetyltryptamin ableiten lässt (siehe Abbildung 49). 


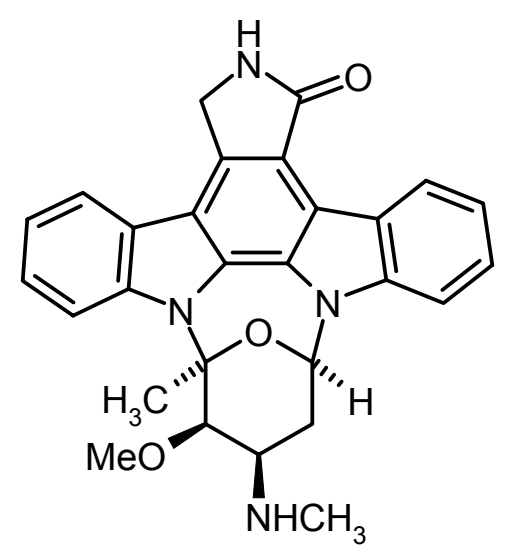

63

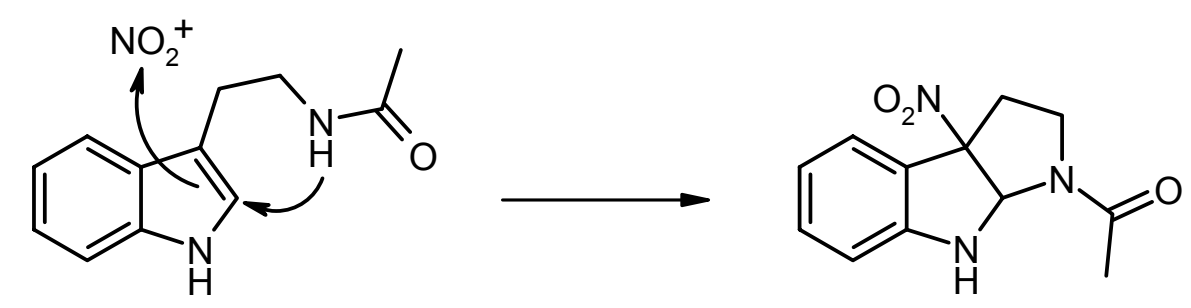

Abbildung 49 Hypothetische Biosynthese von 61.

\subsubsection{Salegentipyrrol B}

Im Dünnschichtchromatogramm zeigte der gelbe Feststoff 64 identisches UV- und Anfärbeverhalten wie 61.

Das ${ }^{1}$ H-NMR-Spektrum zeigte große Ähnlichkeit zu demjenigen von Verbindung 61, es fehlte jedoch das Signal eines Protons im Aromatenbereich. Anhand des Kopplungsmusters konnte auf das Vorliegen eines 1,2,3-trisubstituierten Phenylrings geschlossen werden. Hochauflösende ESI-Massenspektrometrie führte zur Summenformel $\mathrm{C}_{12} \mathrm{H}_{12} \mathrm{~N}_{4} \mathrm{O}_{5}$, welche im Vergleich mit $\mathbf{6 1}$ auf den Austausch eines Protons durch eine Nitrogruppe hindeutete. Aufgrund der geringen Substanzmengen konnten keine weiterführenden 2D-NMR-Experimente durchgeführt werden. Aus den vorhandenen spektroskopischen Daten konnte jedoch ein Strukturvorschlag für Verbindung 64 abgeleitet werden, demzufolge es sich um 1-Acetyl-1,2,3,3a,8,8a-hexahydropyrrolo[2,3-b]-3a,7-dinitro-indol (64) handelte. Substanz $\mathbf{6 4}$ war weder als Naturstoff noch als Syntheseprodukt in der Literatur zu finden. 


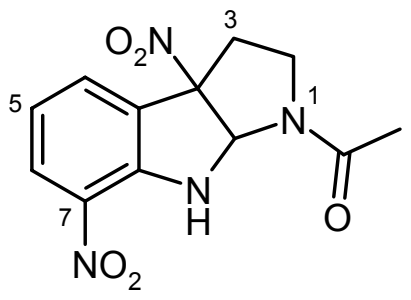

64

\subsubsection{4,5-Dinitro-3-methoxy-phenol}

Die bei $254 \mathrm{~nm}$ UV-löschende, orangefarbene Verbindung $\mathbf{6 5}$ zeigte im APCI-Massenspektrum ein Ion bei $m / z=213[\mathrm{M}-\mathrm{H}]^{-}$, aus dem per Hochauflösung die Zusammensetzung $\mathrm{C}_{7} \mathrm{H}_{6} \mathrm{~N}_{2} \mathrm{O}_{6}$ ermittelt wurde. Dem ${ }^{1} \mathrm{H}-\mathrm{NMR}$-Spektrum waren zwei Signale bei $\delta=8.59$ und $7.55 \mathrm{zu}$ entnehmen, die anhand ihrer charakteristischen Kopplungskonstante einem 1,2,3,5tetrasubstituierten Benzolring zuzuordnen waren. Weiterhin war ein Signal bei $\delta=3.87$ sichtbar, das einer Methoxygruppe zugeschrieben werden konnte. Im IR-Spektrum waren Absorptionsbanden für Phenyl- und Nitrogruppen sichtbar. Mittels 2D-NMR-Spektroskopie konnte die Position der Substituenten am Aromatenring festgelegt werden. Bei Verbindung 65 handelte es sich demzufolge um 4,5-Dinitro-3-methoxy-phenol (65), eine Substanz, die bisher nicht als Naturstoff oder Syntheseprodukt bekannt war.<smiles>COc1cc(O)cc([N+](=O)[O-])c1[N+](=O)[O-]</smiles>

65

\subsubsection{4,6-Dinitro-2-methoxy-phenol}

Das ${ }^{1}$ H-NMR-Spektrum der orangen, UV-Licht bei $254 \mathrm{~nm}$ löschenden Substanz zeigte die gleiche Signalaufspaltung, jedoch unterschiedliche chemische Verschiebungen wie Verbindung 65. Dementsprechend konnte im ${ }^{13} \mathrm{C}-\mathrm{NMR}$-Spektrum dieselbe Zahl an Kohlenstoffatomen detektiert werden. Das EI-Massenspektrum bestätigte ebenfalls die Vermutung, dass es sich um Isomere handelte. Nachdem die Positionen der Substituenten mit Hilfe von 2D-NMR-Spektroskopie bestimmt worden waren, konnte 66 als 4,6-Dinitro-2-methoxy- 
phenol (66) identifiziert werden. Die Struktur konnte zusätzlich durch Vergleich der spektroskopischen Daten mit synthetisch hergestelltem 66 abgesichert werden. Verbindung 66 ist auch unter dem Namen 4,6-Dinitroguaiacol (66) bekannt.<smiles>COc1cc([N+](=O)[O-])cc([N+](=O)[O-])c1O</smiles>

66

4,6-Dinitroguaiacol (66) ${ }^{82}$ und 3,5-Dinitroguaiacol ${ }^{83}$ sind als Stoffwechselprodukte der roten Alge Marginisporum aberrans bekannt und zeigen antimikrobielle Aktivität gegen $B$. subtilis. Außerdem konnte 4,6-Dinitroguaiacol (66) bereits aus getrockneten Chilibohnen Capsicum annuum isoliert werden. ${ }^{84}$

\subsubsection{Sonstige Metaboliten}

\section{2,6-Dimethoxy-1,4-benzochinon}<smiles>COC1=CC(=O)C=C(OC)C1=O</smiles>

67

Das EI-Massenspektrum des orangefarbenen, bei $254 \mathrm{~nm}$ UV-löschenden Feststoffs 67 zeigte das Ion höchster Masse bei $m / z=168$. Im ${ }^{1} \mathrm{H}-\mathrm{NMR}$-Spektrum waren lediglich zwei Singuletts bei $\delta=5.84$ und 3.80 detektierbar. Das ${ }^{13} \mathrm{C}$-NMR-Spektrum zeigte fünf Signale, von denen zwei ( $\delta=186.6$ und 176.7) Carbonylgruppen zugeordnet werden konnten, während die übrigen durch ungewöhnlich hohe Signalintensitäten auffielen. Diese Informationen legten die Vermutung nahe, dass es sich um ein symmetrisches, aus acht Kohlenstoffatomen bestehendes Chinongerüst handelte. Demgemäß konnten die Signale des ${ }^{1} \mathrm{H}$ NMR-Spektrums zwei aromatischen Methingruppen sowie zwei Methoxygruppen zugeordnet werden. Eine Suche in AntiBase ${ }^{30}$ lieferte 2,6-Dimethoxy-1,4-benzochinon (67) als einzigen Strukturvorschlag. Verbindung 67 ist als Pflanzenmetabolit bekannt und zeigt antitumorale Aktivität. ${ }^{85}$ 


\section{3-Indolyl-ethyl-methylether}<smiles>COCCc1c[nH]c2ccccc12</smiles>

68

Die farblose, UV-Licht bei $254 \mathrm{~nm}$ löschende und sich mit Anisaldehyd in der Wärme violett anfärbende Verbindung $\mathbf{6 8}$ zeigte im ${ }^{1} \mathrm{H}-\mathrm{NMR}-$ Spektrum die für Indolderivate typischen Resonanzen im Aromatenbereich. Zusätzlich waren Signale einer Methoxygruppe bei $\delta=3.37$ sowie zweier Methylengruppen bei $\delta=3.66$ und 2.99 erkennbar. Letztere konnten einer Ethandiylgruppe zugeordnet werden, die sich aufgrund der chemischen Verschiebungen zwischen dem Indolgerüst und einem Heteroatom befinden musste. Im IR-Spektrum zeigte 68 eine Absortionsbande bei $1107 \mathrm{~cm}^{-1}$, welche charakteristisch für das Vorliegen einer Ethergruppierung ist. In Kombination mit der per ESI-Hochauflösung bestimmten Summenformel von $\mathrm{C}_{11} \mathrm{H}_{13} \mathrm{NO}$ konnte die Verbindung als 3-Indolyl-ethylmethylether (68) identifiziert werden, das bislang nur als Syntheseprodukt bekannt ist. ${ }^{86}$

\section{Thymin}

Verbindung 69 zeigte im EI-Massenspektrum das für Thymin (69) charakteristische Fragmentierungsmuster. Die im ${ }^{1}$ H-NMR-Spektrum sichtbaren Signale bestätigten diese Zuordnung.<smiles>Cc1c[nH]c(=O)[nH]c1=O</smiles>

69

Zusätzlich konnten Uracil (35) bzw. p-Hydroxybenzoesäure (36) aus Stamm T436 isoliert werden. 


\subsubsection{Isolierte Nitrophenole}

Tabelle 7 Eine Übersicht von isolierten Nitroverbindungen aus dem Stamm T436.

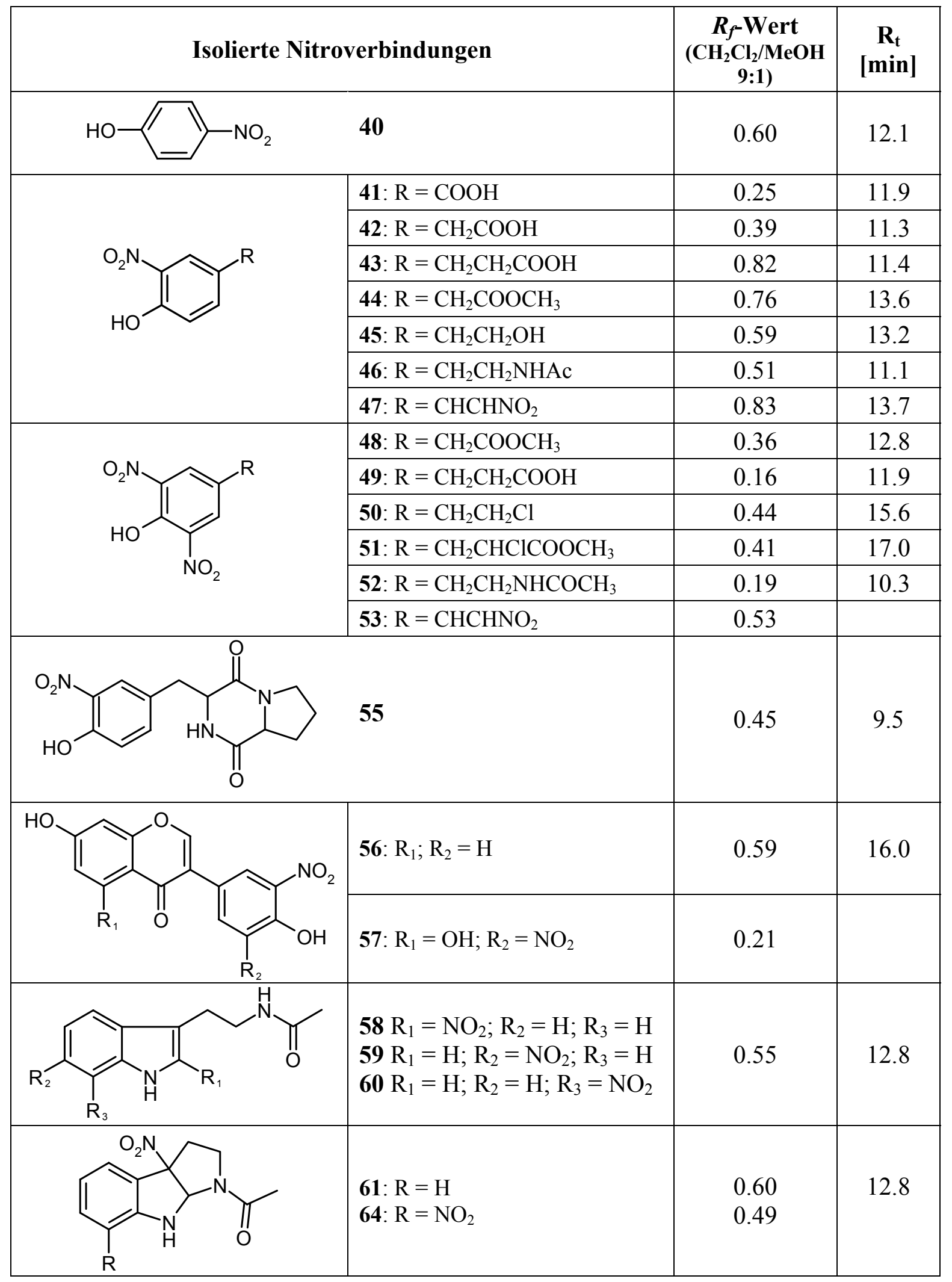




\begin{tabular}{|c|c|c|c|}
\hline \multicolumn{2}{|c|}{ Isolierte Nitroverbindungen } & \multirow{2}{*}{$\begin{array}{c}\boldsymbol{R}_{\boldsymbol{f}} \text {-Wert } \\
\left(\mathrm{CH}_{2} \mathrm{Cl}_{2} / \mathrm{MeOH}\right. \\
\mathbf{9 : 1}) \\
0.51 \\
\end{array}$} & \multirow{2}{*}{$\begin{array}{c}\begin{array}{c}\mathbf{R}_{\mathbf{t}} \\
{[\mathbf{m i n}]}\end{array} \\
14.1\end{array}$} \\
\hline & 65: $\begin{aligned} \mathrm{R}_{1} & =\mathrm{OCH}_{3} ; \mathrm{R}_{2}=\mathrm{H} \\
\mathrm{R}_{3} & =\mathrm{H} ; \mathrm{R}_{4}=\mathrm{NO}_{2}\end{aligned}$ & & \\
\hline $\mathrm{NO}_{2}$ & 66: $\begin{aligned} \mathrm{R}_{1} & =\mathrm{H} ; \mathrm{R}_{2}=\mathrm{OCH}_{3} \\
\mathrm{R}_{3} & =\mathrm{NO}_{2} ; \mathrm{R}_{4}=\mathrm{H}\end{aligned}$ & 0.50 & 14.1 \\
\hline
\end{tabular}

Zusammenfassend lässt sich sagen, dass Nitroverbindungen charakteristische chromatographische und spektroskopische Eigenschaften aufweisen. Die gelben bzw. orangefarbenen Mono- bzw. Dinitrobenzol-Derivate zeigten im Dünnschichtchromatogramm weder beim Besprühen mit Anisaldehyd noch mit Ehrlichs Reagenz eine Farbreaktion. Erst ein spezielles Reagenz für Nitroverbindungen färbt die entsprechenden Zonen an: Mittels Zinn(II)-chlorid-Lösung wurde zunächst die Nitro- zur Aminogruppe reduziert. Diese reagierte anschließend mit 4-Dimethylaminobenzaldehyd unter Ausbildung einer Schiffschen Base, welche sich durch intensive Gelb- bzw. Orangefärbung zu erkennen gibt. Im IR-Spektrum zeigen Nitrogruppen typische Absorptionsbanden bei Wellenlängen von $1510-1570$ und $1320-1350 \mathrm{~cm}^{-1}$. Das unterschiedliche UV-Verhalten von Mono- und Dinitrophenolen kann direkt im Dünnschichtchromatogramm oder mittels eines UVSpektrometers nachgewiesen werden. Erstere löschen im Chromatogramm UV-Licht bei $254 \mathrm{~nm}$ und zeigen ein Absorptionsmaximum bei $\sim 350 \mathrm{~nm}$ (siehe Abbildung 50). Im Gegensatz hierzu weisen Dinitrophenole im Chromatogramm bei $254 \mathrm{~nm}$ gelbe Fluoreszenz sowie im UV-Spektrum eine langwellige Absorption bei $\sim 430 \mathrm{~nm}$ auf. Eine weitere Besonderheit, die bei der spektroskopischen Betrachtungen von Nitroverbindungen beachtet werden muss, ist, dass quartäre aromatische Kohlenstoffatome, die mit Nitrogruppen verknüpft sind, nur bei langen Messzeiten in ${ }^{13} \mathrm{C}$-NMR-Experimenten detektiert werden können. 

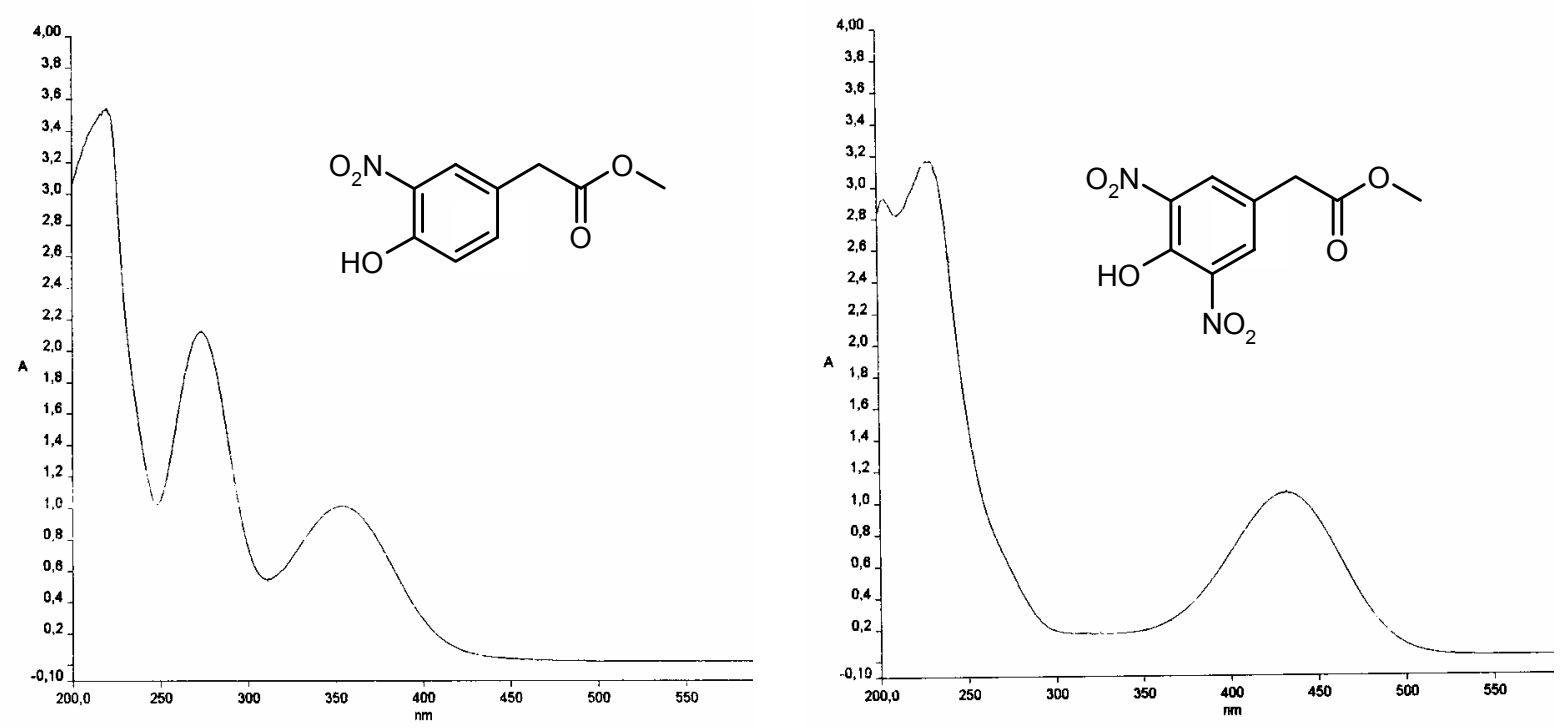

Abbildung 50 UV-Spektrum (MeOH) eines Mononitrobenzol-Derivats (42) im Vergleich mit dem eines zweifach nitrierten Phenols (48).

Bei den bisher durchgeführten Biosyntheseuntersuchungen wurde festgestellt, dass die meisten Nitrogruppen durch Oxidation von Aminogruppen entstehen. So konnte mittels Fütterungsexperimenten nachgewiesen werden, dass $p$-Aminophenylalanin (70) sowohl der Vorläufer des $p$-Nitrophenylserinol-Teils von Chloramphenicol (71) ${ }^{87}$ als auch der Nitrophenyl-Einheit von Aureothin (72) ist. ${ }^{88}$<smiles>Nc1ccc(CC(N)C(=O)O)cc1</smiles>

70<smiles>O=C(N[C@H](CO)[C@H](O)c1ccc([N+](=O)[O-])cc1)C(Cl)Cl</smiles><smiles>CCCCC</smiles><smiles>COc1oc([C@H]2CC(CC(C)Cc3ccc([N+](=O)[O-])cc3)CO2)c(C)c(=O)c1C</smiles>
72 
Bei Biosynthese-Studien an Pyrrolnitrin (73), welches ausgehend von Tryptophan gebildet wird, konnte gezeigt werden, dass das ursprüngliche Indolstickstoffatom N-1 in die entsprechende Aminogruppe umgewandelt wird, welche dann direkt zur Nitrofunktion oxidiert wird. ${ }^{89}$ Van Pee et al. stellten zu einem späteren Zeitpunkt fest, dass das Enzym Chlorperoxidase den letzten Schritt katalysiert. ${ }^{90}$<smiles>NC(Cc1c[nH]c2ccccc12)C(=O)O</smiles>

Tryptophan<smiles>Nc1c(Cl)cccc1-c1c[nH]cc1Cl</smiles><smiles>Cc1c[nH]cc1-c1cccc(Cl)c1[N+](=O)[O-]</smiles>

73

Ein anderer, weitaus seltener beschrittener Biosyntheseweg zur Bildung einer Nitrogruppe ist die direkte Nitrierung. Das einzige bislang beschriebene Beispiel hierfür ist die Verbindung Dioxapyrrolomycin (74), welche in der Arbeitsgruppe von CARTER untersucht wurde. ${ }^{91}$ Durch ${ }^{15} \mathrm{~N}-\mathrm{NMR}$-Experimente und Massenspektrometrie konnte nachgewiesen werden, dass die Nitrogruppe von Dioxapyrrolomycin (74) das gleiche Verhältnis von ${ }^{15} \mathrm{~N}:{ }^{14} \mathrm{~N}$ sowie ${ }^{18} \mathrm{O}:{ }^{16} \mathrm{O}$ wie zugefüttertes $\mathrm{K}^{15} \mathrm{~N}^{18} \mathrm{O}_{3}$ aufwies.<smiles>Clc1cc(Cl)c2c(c1)[C@H](c1cc(Cl)c(Cl)[nH]1)OCO2</smiles><smiles>[13CH3]I</smiles><smiles>O=[N+]([O-])c1c([C@H]2OCOc3c(Cl)cc(Cl)cc32)[nH]c(Cl)c1Cl</smiles>

74

Nicht gänzlich auszuschließend ist ferner die Möglichkeit, dass sich Nitrogruppen durch Oxidation von Nitrosofunktionen während des Aufarbeitungsprozesses bilden. Es konnten bereits mehrfach Nitrosoverbindungen aus natürlichen Quellen isoliert werden. Ein Beispiel hierfür ist 4-Hydroxy-3-nitrosobenzoesäure (75), welche von Li et al. aus Streptomyces murayamaensis isoliert wurde. ${ }^{92}$ 
<smiles>O=Nc1cc(C(=O)O)ccc1O</smiles>

75

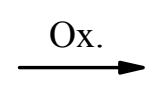<smiles>O=C(O)c1ccc(O)c([N+](=O)[O-])c1</smiles>

41

Um einen tieferen Einblick in die Biosynthese der Nitrogruppen zu erhalten, können Einbaustudien mit $\mathrm{K}^{15} \mathrm{~N}^{18} \mathrm{O}_{3}$ durchgeführt werden. Eine mögliche Biogenese, welche über die Oxidation einer Amino- bzw. Nitrosogruppe verläuft, könnte durch die gezielte Suche nach entsprechenden Vorläufern in Rohextrakten von Stamm T436 nachgewiesen werden.

\subsubsection{ESI-Fragmentierung der aromatischen Nitroverbindungen}

Im Zusammenhang mit der in Kapitel 5 beschriebenen Aufklärung von ESI-Fragmentierungswegen wurden auch die MS/MS-Spektren der isolierten aromatischen Nitroverbindungen näher untersucht. Die leicht ionisierbare Nitrogruppe führte dazu, dass die entsprechenden Substanzen meist nur im negativen Modus sichtbar waren. Um die rasche Erkennung weiterer aromatischer Nitroverbindungen $\mathrm{zu}$ ermöglichen, wurde eine Datenbank mit den erhaltenen MS/MS-Spektren erstellt und die dabei auftretenden charakteristischen Fragmente identifiziert. Die hier gewonnenen Erkenntnisse können bei der Strukturzuordnung neuer Nitroverbindungen eingesetzt werden.

Bei phenolischen, am Aromaten einfach nitrierten Carbonsäuren konnte im negativen Modus häufig die Abspaltung von Kohlendioxid sowie eines $30 \mathrm{Da}$ großen Fragments beobachtet werden.

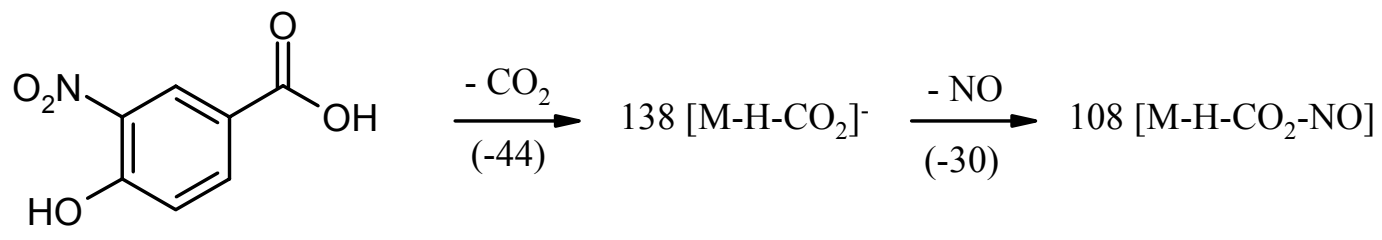




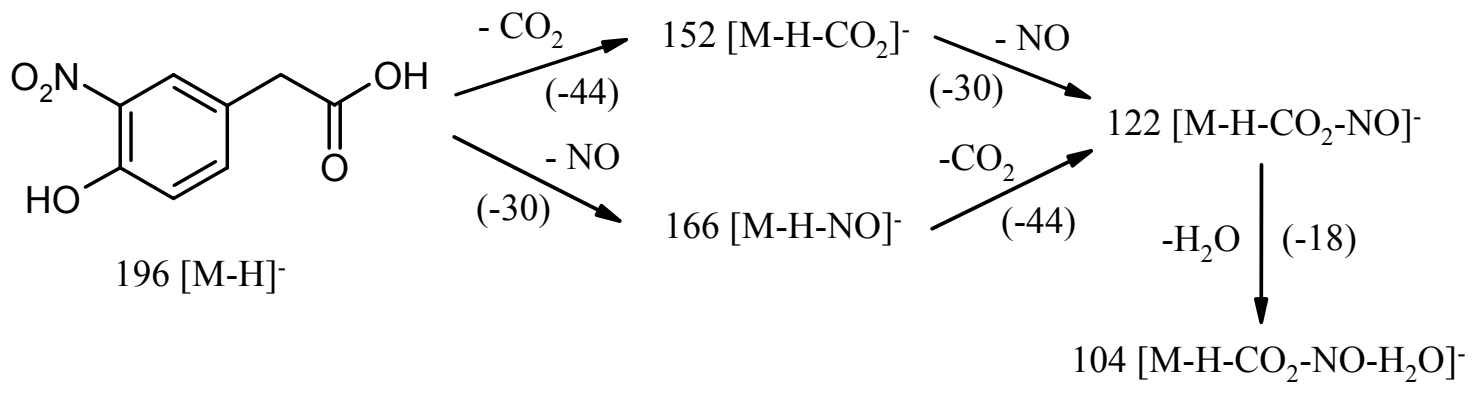

Die Differenz von 30 Masseneinheiten kann durch die Reduktion der aromatischen Nitrozur Aminofunktion erklärt werden. Dieses Phänomen wurde bereits Anfang der achtziger Jahre bei auf chemischer Ionisation (CI) beruhenden MS-Experimenten untersucht. ${ }^{93,94,95}$ Zusätzlich wurde bei APCI-basierten Studien in Abhängigkeit vom verwendeten Lösungsmittel das um $30 \mathrm{Da}$ leichtere Fragment im positiven Modus detektiert. ${ }^{96}$ Eine andere Begründung, die das Auftreten dieses Spaltprodukts erklärt, geht von einer NO-Eliminierung aus, welche bereits aus EI-Experimenten bekannt ist. ${ }^{97}$ Diese Theorie wird dadurch gestützt, dass in den Full-scan-Spektren keine [M-H-30]'-Ionen detektiert werden konnten, diese vielmehr erst nach dem Fragmentierungsprozess auftraten.

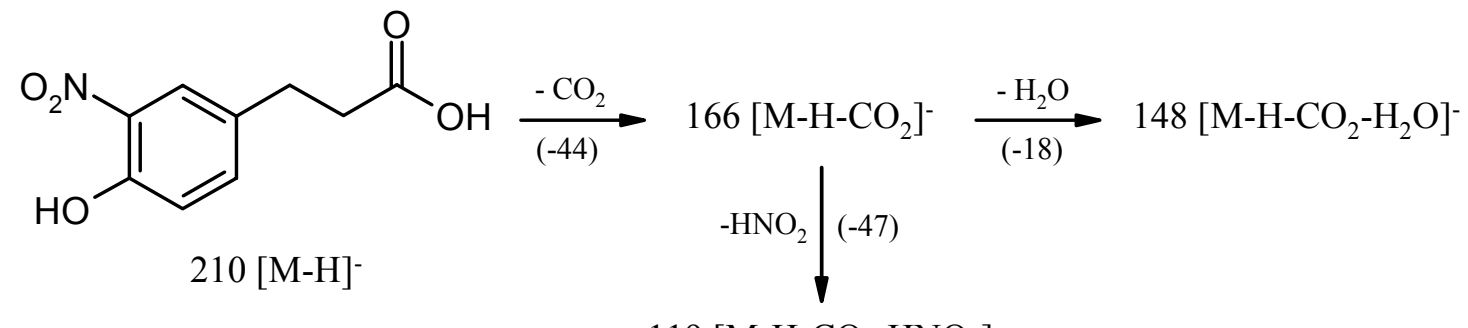
$119\left[\mathrm{M}-\mathrm{H}-\mathrm{CO}_{2}-\mathrm{HNO}_{2}\right]^{-}$

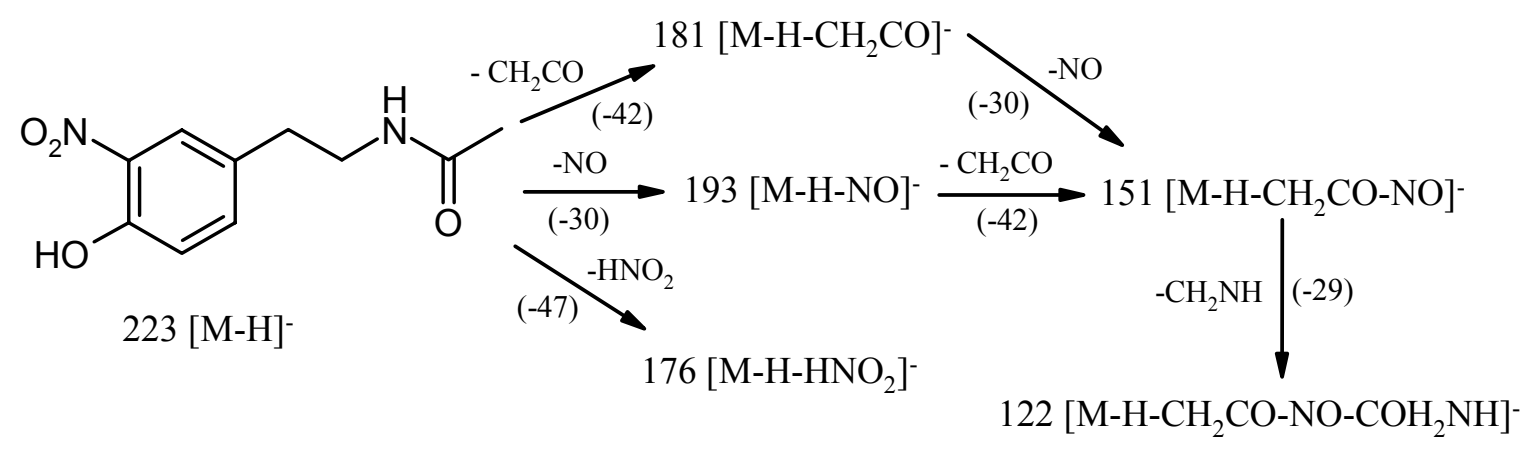

In mehreren Fällen konnte ein Massenverlust von 47 Einheiten detektiert werden, was auf die Abspaltung eines Moleküls $\mathrm{HNO}_{2}$ hindeutete.

Im Falle der bisher untersuchten phenolischen Dinitroverbindungen kam es zu ähnlichen Fragmentierungsmustern wie bei einfach nitrierten Substanzen. 
Verfügt das vorliegende Nitrophenolderivat über eine leicht abspaltbare Gruppe wie z. B. Chlor, wurde diese zuerst (hier in Form von Chlorwasserstoff) abgespalten. Anschließend erfolgte die Eliminierung von NO.<smiles>O=[N+]([O-])c1cc(CCCl)cc([N+](=O)[O-])c1O</smiles>

Liegt hingegen unsubstituiertes Nitrophenol vor, kommt es lediglich zur Abspaltung von Stickstoffmonoxid.<smiles></smiles>

Im positiven Modus konnten nur solche Nitroverbindungen detektiert werden, welche über eine leicht ionisierbare funktionelle Gruppe verfügen. In diesen Fällen konnte stets die Eliminierung eines 46 D schweren Fragments beobachtet werden, wobei es sich vermutlich um Stickstoffdioxid handelt.<smiles>CC(=O)NCCc1c[nH]c2c([N+](=O)[O-])cccc12</smiles>
$248[\mathrm{M}+\mathrm{H}]^{+}$

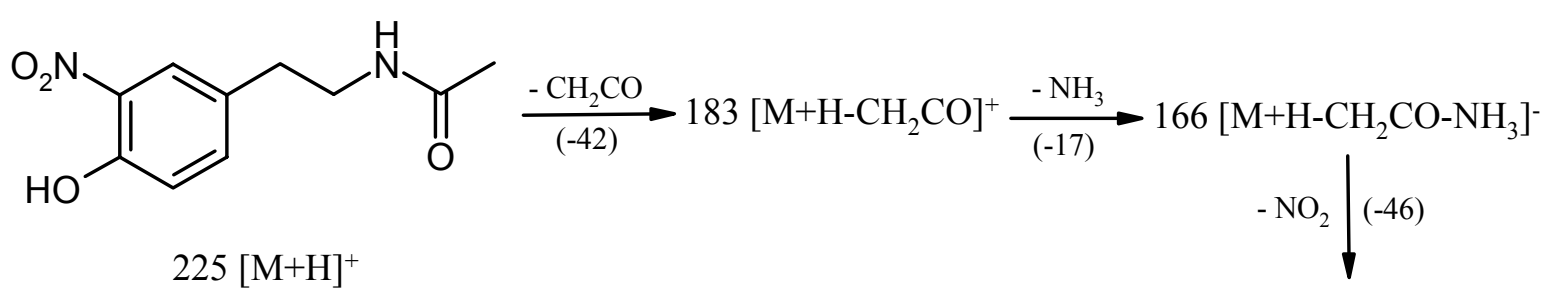
$143\left[\mathrm{M}+\mathrm{H}-\mathrm{CH}_{2} \mathrm{CO}-\mathrm{NH}_{3}-\mathrm{NO}_{2}\right]^{-}$ 


\subsection{Actinomyces sp. T426}

Der Stamm T426 wurde aus litoralem Sediment in Feuerland isoliert. Beim biologischen Screening des Rohextraktes gegen verschiedene Organismen konnte eine cytotoxische Aktivität gegen verschiedene Zelllinien gefunden werden.

\subsubsection{Tryptophol und $p$-Hydroxybenzoesäuremethylester}

Im EI-Massenspektrum zeigte die farblose Flüssigkeit $\mathbf{7 6}$ das Ion höchster Masse bei $m / z=161$. Anhand der typischen chemischen Verschiebungen sowie Signalaufspaltungen im ${ }^{1}$ H-NMR-Spektrum bei $\delta=7.62,7.37,7.20,7.12$ und 7.08 konnte auf Indol als Grundstruktur geschlossen werden. Im Dünnschichtchromatogramm zeigte 76 eine violette Farbreaktion beim Besprühen mit Ehrlichs Reagenz in der Wärme, was das Vorliegen eines Indolderivats zusätzlich bestätigte. Weiterhin waren Signale im aliphatischen Bereich bei $\delta=3.90$ und $3.03 \mathrm{zu}$ erkennen, die einer an ein Heteroatom gebundenen Ethylengruppe zugeordnet werden konnten. Eine Suche in Antibase ${ }^{30}$ mit der ermittelten Molmasse sowie den aus der NMR-Spektroskopie erhaltenen Strukturfragmenten ergab, dass es sich bei 76 um Tryptophol (76) handeln musste.<smiles>OCCc1c[nH]c2ccccc12</smiles>

76<smiles>COC(=O)c1ccc(O)cc1</smiles>

77

Das EI-MS-Spektrum des weißen Feststoffs 77 zeigte das für $p$-Hydroxy-benzoesäuremethylester (77) charakteristische Fragmentierungsmuster. Ein Vergleich der ${ }^{1} \mathrm{H}-\mathrm{NMR}-\mathrm{Spek}-$ tren von 77 mit demjenigen von $p$-Hydroxybenzoesäure (36) zeigte eine Übereinstimmung der Signale im aromatischen Bereich, wobei Verbindung 77 die zusätzliche Resonanz einer Methoxygruppe bei $\delta=3.83$ aufwies. Somit wurde der sich aus der EI-Datenbank NIST ergebende Strukturvorschlag bestätigt. 


\subsection{Actinomyces sp. T421}

Der Stamm T421 wurde ebenfalls aus litoralem Sediment in Feuerland isoliert. Im biologischen Screening zeigte der Rohextrakt cytotoxische Aktivität gegen einige Zelllinien.

\subsection{1 trans-3-Methylthioacrylsäure}

Der UV-löschende, farblose Feststoff 78 zeigte im EI-Massenspektrum ein Ion höchster Masse bei $m / z=118$. Im ${ }^{1} \mathrm{H}-\mathrm{NMR}-S p e k t r u m$ waren neben einer Methylgruppe bei $\delta=2.37$ zwei weitere Signale bei $\delta=7.88$ und 5.66 sichtbar, die im Bereich von Doppelbindungsprotonen lagen und von denen eines aufgrund seiner chemischen Verschiebung an ein Heteroatom gebunden sein musste. Nach einer Suche in AntiBase ${ }^{30}$ sowie einem Vergleich der spektroskopischen Daten mit Literaturwerten konnte 78 als trans-3-Methylthioacrylsäure (78) identifiziert werden.<smiles>CS/C=C/C(=O)O</smiles>

78

Bei systematischen Untersuchungen von SURETTE und VINING ${ }^{98}$ konnte 1976 erstmals ein Zusammenhang zwischen der Bildung von 78 und der Konzentration an L-Methionin im Kulturmedium von Streptomyces lincolnensis festgestellt werden. Für die Biosynthese von 78 wird der in Abbildung 51 dargestellte Mechanismus vermutet.

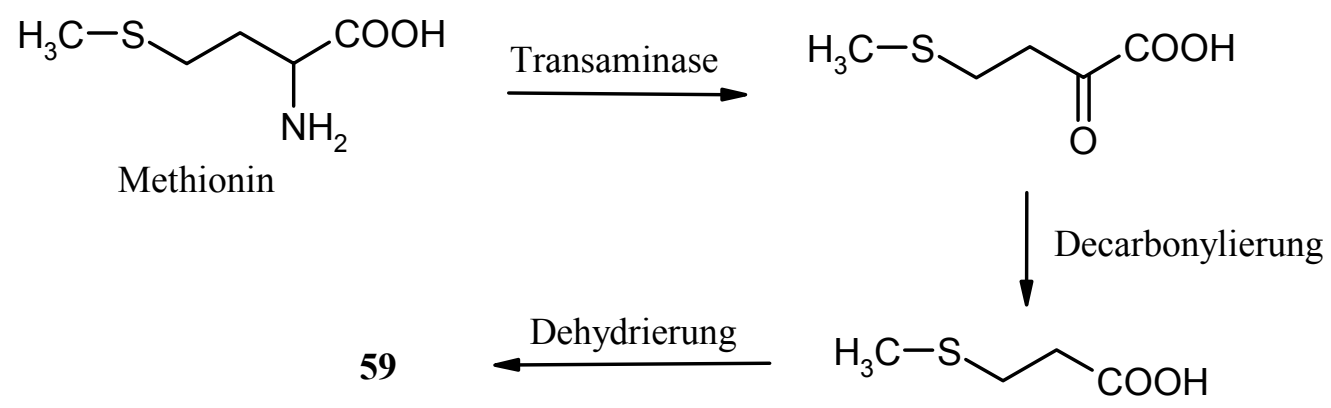

Abbildung 51 Bildung von $\mathbf{7 8}$ aus Methionin nach SURETTE und VINING. ${ }^{98}$ 


\subsubsection{Benzoesäure und Phenylessigsäure}

Die Verbindung 79 fiel im Dünnschichtchromatogramm durch UV-Löschung bei $254 \mathrm{~nm}$ auf, wies jedoch keine Anfärbung beim Besprühen mit Anisaldehyd auf. Durch Auswertung des ${ }^{1} \mathrm{H}-\mathrm{NMR}-\mathrm{Spektrums}$ sowie des charakteristischen EI-MS-Fragmentierungsmusters konnte die Substanz rasch als Benzoesäure (79) identifiziert werden.<smiles>O=C(O)c1ccccc1</smiles>

79<smiles>O=C(O)Cc1ccccc1</smiles>

80

Der ebenfalls farblose Feststoff $\mathbf{8 0}$ zeigte das gleiche UV- und Anfärbeverhalten wie Verbindung 79. Im EI-Massenspektrum war ein Ion höchster Masse bei $m / z=136$ erkennbar. Die Massendifferenz von $\Delta m / z=14$ zwischen den Substanzen 80 und 79 legte die Vermutung nahe, dass 80 über eine zusätzliche Methyl- oder Methylengruppe verfügte. Dieser Befund konnte im ${ }^{1} \mathrm{H}-\mathrm{NMR}-$ Spektrum bestätigt werden. Es handelte sich bei der vorliegenden Verbindung folglich um in unserem Arbeitskreis bereits häufig isolierte Phenylessigsäure $(\mathbf{8 0})$.

\subsection{Stamm T846}

Der Stamm T846 wurde aus einer Wasserprobe des Humboldt-Stroms vor der chilenischen Küste isoliert. Im Dünnschichtchromatogramm fiel eine ausgeprägte, stark UV-löschende Zone auf, die sich weder mit Anisaldehyd noch mit Ehrlich-Reagenz anfärbte.

\subsubsection{3-Indolylcarbonsäure und 3-Indolylcarbaldehyd}

Das farblose, sich mit Anisaldehyd rot sowie mit Ehrlichs Reagenz violett anfärbende Ö1 wies UV-Löschung bei 254 nm auf. EI-Massen- und ${ }^{1}$ H-NMR-Spektrum zeigten identische Signale wie diejenigen von 33, weswegen die vorliegende Substanz rasch als 3-Indolylcarbonsäure (33) identifiziert werden konnte. 


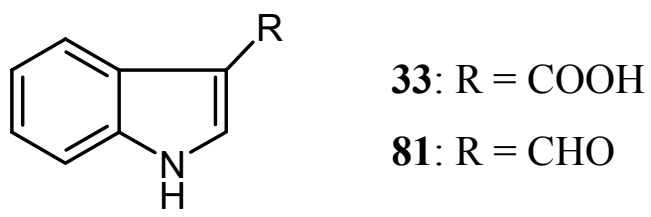

Die ebenfalls in Form eines farblosen Feststoffs isolierte Verbindung $\mathbf{8 1}$ wies gleiches UVund Anfärbeverhalten auf wie 33. Das ${ }^{1} \mathrm{H}-\mathrm{NMR}-$ Spektrum deutete ebenso auf das Vorliegen eines Indolgerüsts hin, während im EI-Massenspektrum eine um 16 Einheiten geringere Masse als bei 33 detektiert wurde. Eine Datenbanksuche in NIST wies auf 3Indolylcarbaldehyd (81) hin. Ein Vergleich der ${ }^{1} \mathrm{H}-\mathrm{NMR}-\mathrm{Daten}$ mit dem in unserer Arbeitsgruppe vorhandenen Spektrum von $\mathbf{8 1}$ bestätigte diesen Befund.

\subsubsection{3-(Methylthio)propansäure}

Der farblose Feststoff 82 zeigte im Dünnschichtchromatogramm UV-Löschung und keine Anfärbung beim Besprühen mit Anisaldehyd. Das ${ }^{1} \mathrm{H}-\mathrm{NMR}-$ Spektrum wies ein mit $\mathrm{D}_{2} \mathrm{O}$ austauschbares Signal bei $\delta=10.76$ auf. Weiterhin fanden sich Resonanzen für zwei benachbarte Methylengruppen bei $\delta=2.74$ und 2.66 sowie eine für Methylgruppe bei $\delta=2.11$. Mit diesen NMR-Daten wurde eine Datenbanksuche in AntiBase ${ }^{30}$ durchgeführt, welche 3-(Methylthio)propansäure (82) als Strukturvorschlag lieferte. Die vollständige Übereinstimmung der spektroskopischen Daten mit in unserer Arbeitsgruppe vorhandenen Referenzwerten bestätigte diesen Hinweis. Das Auftreten von Verbindung 82, welche ein biosynthetisches Zwischenprodukt bei der Bildung von 3-(Methylthio)acrylsäure (78) darstellt, kann als Indiz für die Korrektheit des in Abbildung 51 angeführten Mechanismus angeführt werden. ${ }^{98}$ Die $S$-methylierte Form von 82 (DMSP) ist vor allem als Stoffwechselprodukt von Phytoplankton bekannt. ${ }^{99}$ Im Zuge bakteriellen Abbaus wird sie in Dimethylsulfid (DMS) umgewandelt. DMSP sowie DMS spielen eine wichtige Rolle im globalen Schwefelkreislauf. ${ }^{100}$

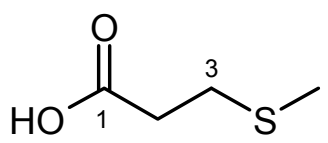




\subsubsection{1,3,5-Triazin}

Im Dünnschichtchromatogramm war 83 durch UV-Löschung bei $254 \mathrm{~nm}$ detektierbar, färbte sich jedoch mit keinem der gängigen Sprühreagenzien an. Die Substanz wies eine sehr hohe Polarität auf, welche sich im $R_{f}$-Wert von $0.21 \mathrm{im}$ Laufmittelgemisch $\mathrm{CH}_{2} \mathrm{Cl}_{2} /$ $\mathrm{MeOH}$ 9:1 wiederspiegelte.

Weder EI-, ESI- noch DCI-Massenspektrum zeigten das Auftreten eines Molekülions an. Im ${ }^{1}$ H-NMR-Spektrum war lediglich ein Singulett bei $\delta=8.53$ erkennbar. Ebenso fand sich im ${ }^{13} \mathrm{C}$-NMR-Spektrum nur eine Resonanz bei $\delta=170.5$. Davon ausgehend wurde vermutet, dass es sich bei der isolierten Verbindung um 1,3,5-Triazin (83) handelte. Ein Vergleich der NMR-Daten mit denjenigen von kommerziell erhältlichem 1,3,5-Triazin (83) bestätigte die Vermutung. Die niedermolekulare, heterocyclische Verbindung $\mathbf{8 3}$ war bis jetzt lediglich als Syntheseprodukt bekannt.

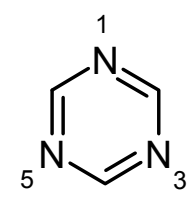

83

\subsection{Stamm T406}

Der aus Pinguinexkrementen isolierte, psychrophile Stamm T406 zeigte bei einer Temperatur von $4{ }^{\circ} \mathrm{C}$ optimales Wachstum. Im Dünnschichtchromatogramm des Rohextrakts waren mehrere gelbe bzw. UV-löschende Zonen auffällig, von denen eine nach Besprühen mit Anisaldehyd eine dunkelviolette Farbreaktion einging. Im biologischen Screening zeigte der Rohextrakt cytotoxische Aktivität gegen Nematoden sowie einige Zelllinien.

\subsubsection{2-(Indol-3-ylmethyl)-indol-3-ylethanol}

Verbindung 84 fiel im Dünnschichtchromatogramm durch UV-Löschung bei $254 \mathrm{~nm}$ und Violettfärbung beim Besprühen mit Anisaldehyd auf. In Lösung war 84 farblos, während die Substanz im trockenen Zustand eigentümlicherweise eine violette Farbe aufwies. 


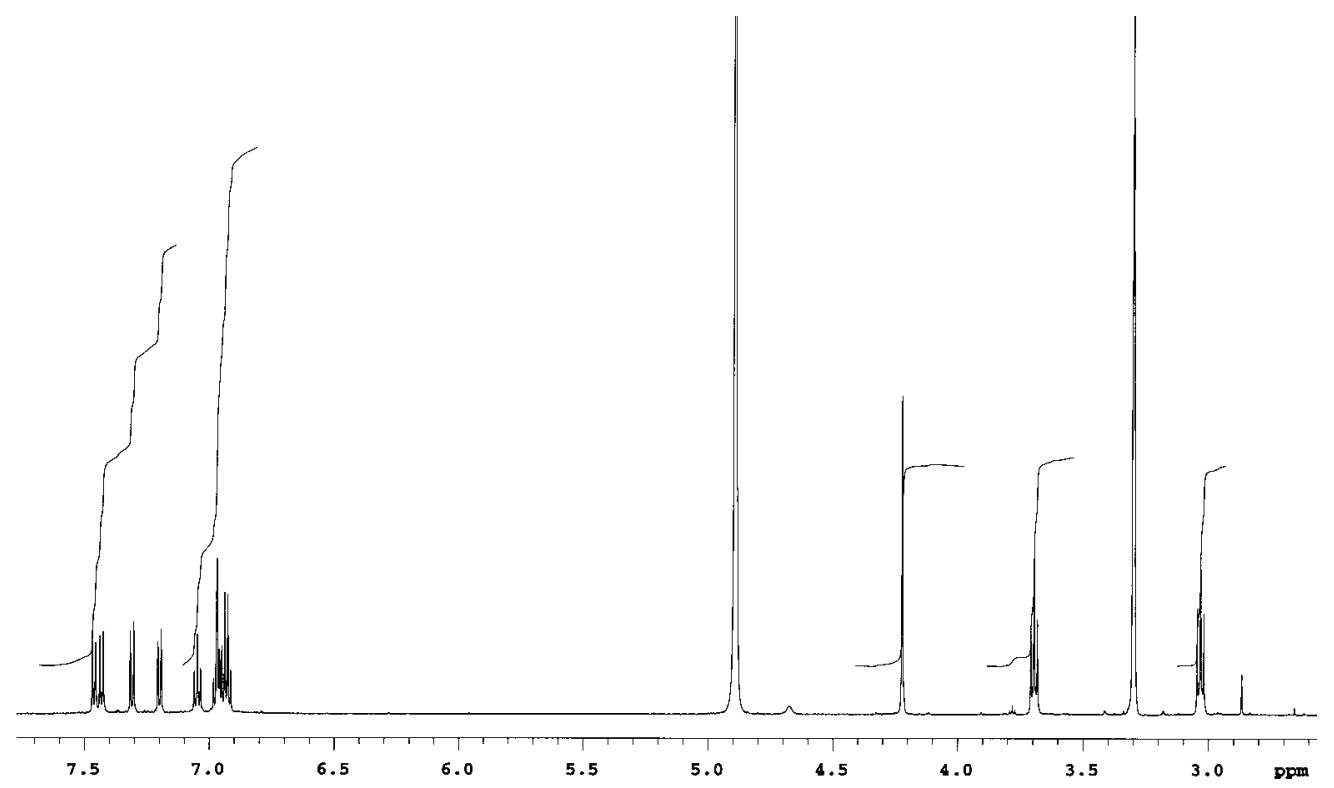

Abbildung $52{ }^{1} \mathrm{H}-\mathrm{NMR}-\mathrm{Spektrum}\left(600 \mathrm{MHz}, \mathrm{CD}_{3} \mathrm{OD}\right)$ von 2-(Indol-3-ylmethyl)-indol3-ylethanol (84).

Im DCI-Massenspektrum von 84 war ein Ion bei $m / z=291[\mathrm{M}+\mathrm{H}]^{+}$sichtbar, aus dem sich durch Hochauflösung die Summenformel $\mathrm{C}_{19} \mathrm{H}_{18} \mathrm{~N}_{2} \mathrm{O}$ ableiten ließ. Das ${ }^{1} \mathrm{H}-\mathrm{NMR}-\mathrm{Spek}$ trum zeigte neun aromatische Signale bei $\delta=7.46,7.43,7.31,7.20,7.05,6.97,6.96,6.94$ und 6.92 sowie Resonanzen für drei Methylengruppe bei $\delta=4.22,3.69$ und 3.04. Dem H,H-COSY-Spektrum konnten die in Abbildung 53 dargestellten Korrelationen von acht aromatischen Protonen sowie zweier Methylengruppen untereinander entnommen werden. Hieraus ergab sich, dass zwei 1,2-disubstituierte Phenylsysteme sowie eine Ethylengruppe im Molekül vorlagen. Aufgrund der chemischen Verschiebung musste letztere an mindestens ein Heteroatom gebunden sein.
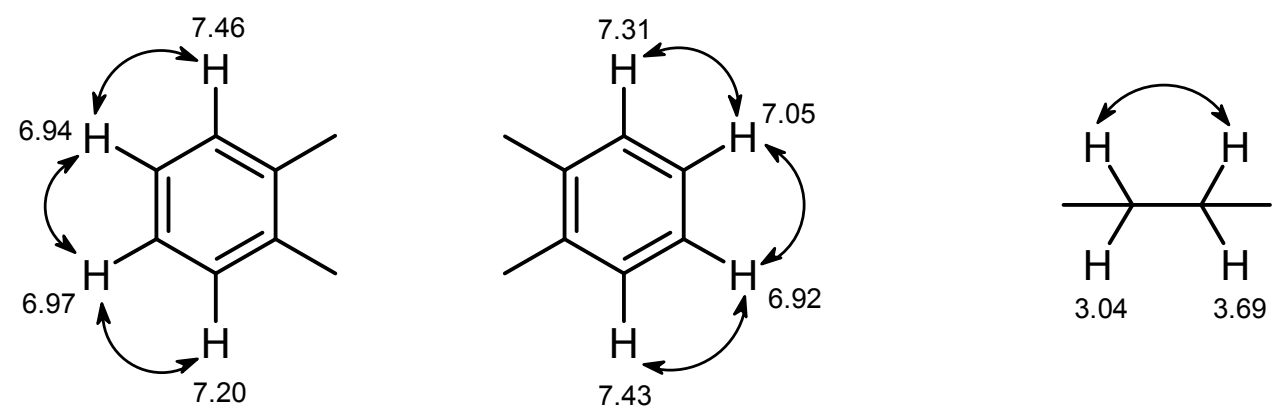

Abbildung 53 Mittels H,H-COSY-Korrelationen abgeleitete Strukturfragmente von 2(Indol-3-ylmethyl)-indol-3-ylethanol (84). 
Das ${ }^{13}$ C-NMR-Spektrum von 84 zeigte entsprechend der Summenformel neunzehn Signale. Über ein HMBC-Experiment (siehe Abbildung 54) konnten die erhaltenen Fragmente miteinander verknüpft und die Struktur somit aufgeklärt werden. Es handelte sich um in der Literatur bisher als Naturstoff nicht bekanntes 2-(Indol-3-ylmethyl)-indol-3-ylethanol (84).

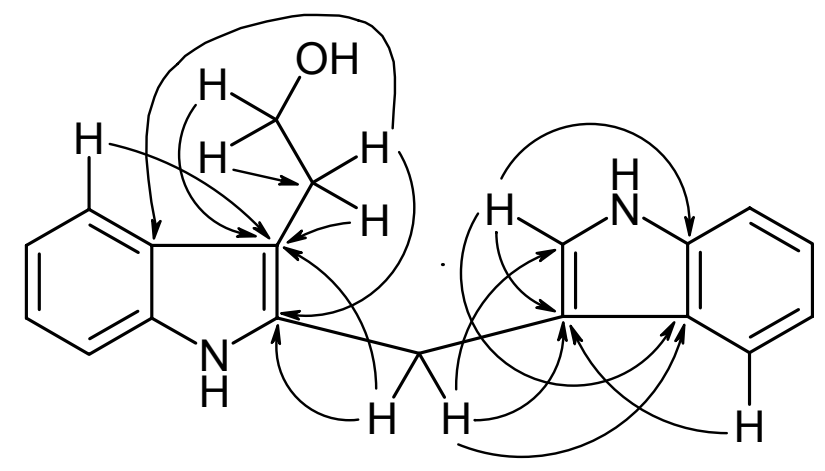

Abbildung 54 Mittels HMBC-Korrelationen abgeleitete Strukturfragmente von 2-(Indol3-ylmethyl)-indol-3-ylethanol (84).<smiles>[R]c1c(Cc2c[nH]c3ccccc23)[nH]c2ccccc12</smiles>

84: $\mathrm{R}=\mathrm{CH}_{2} \mathrm{CH}_{2} \mathrm{OH}$

85: $\mathrm{R}=\mathrm{CH}_{2} \mathrm{CHOHCOOH}$

86: $\mathrm{R}=\mathrm{CH}_{2} \mathrm{COOH}$

Die strukturell ähnlich aufgebaute Substanz 85 ist bereits als Metabolit von Malassezia furfur beschrieben worden, einer Hefe, die die menschliche Haut besiedelt und für seborrhoeische Ekzeme verantwortlich gemacht wird. ${ }^{101}$ Der Phenoloxidase-Inhibitor 85 wird zur Behandlung von Hyperpigmentationen sowie bei malignen und semimalignen Melanozyten angewendet. Bei Verbindung 86, welche 1987 aus dem thermophilen Archaebakterium Sulfolobus acidocaldarius isoliert werden konnte, handelt es sich um die oxidierte Form von $84 .^{102}$

\subsubsection{Ubichinon Q7}

Die als gelbes Ö1 anfallende Substanz 87 war im Dünnschichtchromatogramm $\left(R_{f}=0.95\right.$, $\mathrm{CH}_{2} \mathrm{Cl}_{2} / \mathrm{MeOH}$ 95:5) durch eine bei $254 \mathrm{~nm}$ UV-löschend, sich mit Anisaldehyd in der Wärme violett anfärbende Zone sichtbar. 
Im ${ }^{1} \mathrm{H}-\mathrm{NMR}-$ Spektrum von 87 waren sieben Methingruppen bei $\delta=5.10-4.91$, zwei Methoxygruppen bei $\delta=3.97$ und 3.96, dreizehn Methylengruppen bei $\delta=3.16,2.05-1.94$ sowie neun Methylgruppen bei $\delta=1.99,1.71,1.65,1.56$ und 1.55 zu erkennen. Aromatische Protonen konnten hingegen nicht detektiert werden. Das Spektrum wies eine große Ähnlichkeit zu dem von Ubichinon Q8 (27, siehe Kapitel 6.1.5) auf. Anhand der Anzahl der im ${ }^{1}$ H-NMR-Spektrum sichtbaren Protonen wurde darauf geschlossen, dass es sich um Ubichinon Q7 (87) handelte.<smiles>COC1=C(OC)C(=O)C(C/C=C(\C)CC/C=C(\C)CC/C=C(\C)CC/C=C(\C)CC/C=C(\C)CC/C=C(\C)CCC=C(C)C)=C(C)C1=O</smiles>

87

\subsubsection{SS-43405e}

Verbindung 88 war im Dünnschichtchromatogramm durch die intensive orange Farbe und UV-Löschung bei $254 \mathrm{~nm}$ detektierbar, färbte sich jedoch mit keinem der gängigen Sprühreagenzien an. Die Substanz konnte lediglich in einer sehr geringen Ausbeute von $0.02 \mathrm{mg} / \mathrm{l}$ als gelber Feststoff isoliert werden.

Das ${ }^{1} \mathrm{H}-\mathrm{NMR}-$ Spektrum von $\mathbf{8 8}$ wies ein mit $\mathrm{D}_{2} \mathrm{O}$ austauschbares Signal bei $\delta=12.92$ sowie Resonanzen von fünf aromatischen $(\delta=8.03,7.80,7.65,7.34$ und 6.24) und einer aliphatischen $(\delta=2.70)$ Methingruppe auf. Weiterhin waren Signale für eine diastereotope Methylengruppe bei $\delta=1.95$ und 1.76 sowie zwei Methylgruppen bei $\delta=1.41$ und 0.96 detektierbar. Im ESI-Massenspektrum zeigte 88 ein Ion bei $m / z=363[\mathrm{M}+\mathrm{H}]^{+}$. Eine Suche in AntiBase ${ }^{30}$ und anschließender Vergleich der NMR-Daten mit Spektren bekannter Verbindungen führte zur Identifizierung von $\mathbf{8 8}$ als SS-43405e (88). Es handelt sich hierbei um einen Vertreter aus der Klasse der Pluramycine, welcher antimikrobielle und antitumorale Aktivität besitzt. Letztere ist auf die selektive Ausbildung von DNA-Bindungen zurückzuführen. ${ }^{103}$ 
<smiles>CCC(C)c1cc(=O)c2c(C)cc3c(c2o1)C(=O)c1c(O)cccc1C3=O</smiles>

88

\subsubsection{Indolderivate}

\section{Tryptophol}

Mittels EI-Massen- und ${ }^{1}$ H-NMR-Spektroskopie konnte das farblose, UV-Licht bei $254 \mathrm{~nm}$ löschende und sich mit Anisaldehyd rot anfärbende Öl rasch als Tryptophol (76, siehe Kapitel 6.3.1) identifiziert werden.<smiles>OCCc1c[nH]c2ccccc12</smiles>

76<smiles>CC(=O)OCCc1c[nH]c2ccccc12</smiles>

89

\section{2-(3-Indolyl)ethylacetat}

Der farblose Feststoff 89 löschte im Dünnschichtchromatogramm UV-Licht bei $254 \mathrm{~nm}$.

Die Verbindung färbte sich mit Anisaldehyd rot sowie mit Ehrlichs Reagenz charakteristisch violett an, was auf das Vorliegen eines Indolderivates hindeutete.

Diese Vermutung wurde durch das ${ }^{1} \mathrm{H}-\mathrm{NMR}-$ Spektrum von 89 bestätigt, welches ein für Indolgerüste typisches Signalmuster aufwies. Zusätzlich waren Signale für zwei Methylengruppen bei $\delta=4.35$ und 3.10 sowie eine Acetylgruppe bei $\delta=2.05 \mathrm{zu}$ erkennen. Erstere wurden aufgrund ihrer chemischen Verschiebungen und anhand des Aufspaltungsmusters einer Ethylengruppe zugeordnet, welche an das Indolgerüst gebunden und zusätzlich mit einem Heteroatom substituiert vorliegen musste. Das EI-Massenspektrum von 89 zeigte ein Ion höchster Masse bei $m / z=203$. Mittels dieser Informationen wurde die Verbindung als 2-(3-Indolyl)ethylacetat (89) identifiziert. 
Substanz 89 wurde erstmal von Glombitza et al. als Metabolit der Hefearten Hansenula anomala und Torulopsis utilis beschrieben. ${ }^{104} \mathrm{Zu}$ einem späteren Zeitpunkt konnte die Verbindung aus dem Pilz Ceratocystis fagacearum isoliert werden. Als biosynthetischer Ursprung wurde Tryptophan bzw. Tryptophol nachgewiesen. ${ }^{105}$

\subsubsection{Cyclo(Dehydroalanyl-Isoleucyl)}

Verbindung 90 fiel im Dünnschichtchromatogramm $\left(R_{f}=0.51, \mathrm{CH}_{2} \mathrm{Cl}_{2} / \mathrm{MeOH} 9: 1\right)$ durch UV-Löschung bei $254 \mathrm{~nm}$ sowie durch Orangefärbung beim Besprühen mit Anisaldehyd sowie Ehrlichs Reagenz auf. Die Substanz konnte in Form eines farblosen Feststoffs isoliert werden.

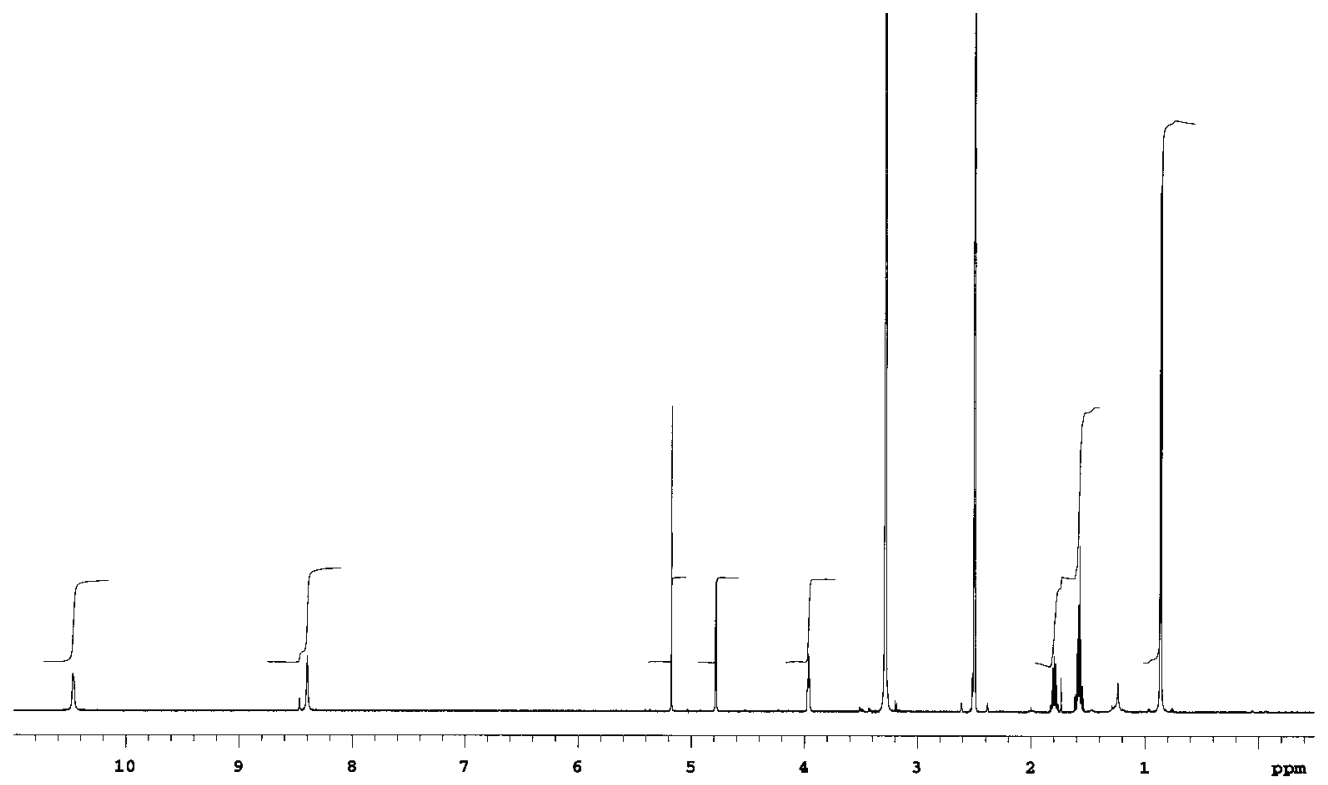

Abbildung $55{ }^{1} \mathrm{H}-\mathrm{NMR}-$ Spektrum (600 MHz, [D $]$ DMSO) von Cyclo(DehydroalanylIsoleucyl) (90).

Das ${ }^{1} \mathrm{H}-\mathrm{NMR}-$ Spektrum wies zwei verbreiterte, mit $\mathrm{D}_{2} \mathrm{O}$ austauschbare Signale bei $\delta=10.47(1 \mathrm{H})$ und $8.40(1 \mathrm{H})$ auf, welche auf das Vorliegen von NH- und/oder OHGruppen hindeuteten. Zwei im olefinischen Bereich bei $\delta=5.18$ und 4.79 auftretende, für jeweils ein Proton integrierende Singuletts konnten anhand der zugehörigen HSQCKorrelationen einer Methylengruppe zugeschrieben werden. Darüber hinaus konnte die Resonanz einer Methingruppe bei $\delta=3.96$ detektiert werden, welche aufgrund ihrer chemischen Verschiebung mit einem Heteroatom substituiert sein musste. Zusätzlich konnten Signale für eine Methingruppe bei $\delta=1.80$, zwei homotope Methylgruppen bei $\delta=0.86$ 
sowie eine Methylengruppe bei $\delta=1.58$ detektiert werden. $\operatorname{Im}{ }^{13} \mathrm{C}$-NMR-Spektrum waren insgesamt acht Kohlenstoffatome zu erkennen, wobei Resonanzen bei $\delta=166.4$ und 158.2 auf die Anwesenheit von Carbonylgruppen im Molekül hindeuteten. Die bei $\delta=134.7$ und 98.9 auftretenden Signale konnten Kohlenstoffatomen einer Doppelbindung zugeordnet werden, welche sich in Konjugation mit einer Carbonylfunktion befinden musste. Da die massenspektroskopische Untersuchung von 90 keine auswertbaren Daten lieferte, wurde zur weiteren Strukturzuordnung auf 2D-NMR-Experimente zurückgegriffen. Deren Auswertung legte nahe, dass ein Diketopiperazin isoliert wurde, welches über eine exocyclische Doppelbindung verfügte. Ein Vergleich der NMR-Daten führte zur Verbindung Cyclo(Dehydroala-Isoleu) (90), welche bereits zuvor in unserer Arbeitsgruppe isoliert werden konnte. ${ }^{106}$<smiles>CC(C)C1NC(=O)C(=[V])NC1=O</smiles>

90

Das Isomer von 90, Cyclo(Dehydroala-Leu) wurde zum ersten Mal aus Kulturen von Penicillium sp. F70614 isoliert und zeigt einen inhibitorischen Effekt gegenüber $\alpha$-Glucosidasen. ${ }^{107}$ Diese Enzyme katalysieren die Spaltung der $\alpha$-glycosidischen Bindung im letzten Schritt des Kohlenhydratabbaus und sind als potenzielle Therapeutika zur Behandlung von Diabetes mellitus im Gespräch. ${ }^{108}$

\subsubsection{2-Phenylethanol}

Die farblose Flüssigkeit 91 zeigte weder beim Ansprühen mit Anisaldehyd noch mit Ehrlichs Reagenz eine Farbreaktion. Die Substanz fiel im Dünnschichtchromatogramm durch UV-Löschung bei $254 \mathrm{~nm}$ auf und konnte in einer Ausbeute von $0.9 \mathrm{mg} / \mathrm{l}$ isoliert werden.

Das EI-Massenspektrum von 91 zeigte das Ion höchster Masse bei $m / z=122$. Im ${ }^{1} \mathrm{H}$ NMR-Spektrum waren charakteristische Signale für einen monosubstituierten Benzolring sowie für zwei Methylengruppen bei $\delta=3.83$ und 2.86 vorhanden. Letztere konnten aufgrund ihrer chemischen Verschiebung und anhand des Aufspaltungsmusters einer Ethylengruppe zugeordnet werden, welche an das aromatische System gebunden und mit einem 
Heteroatom substituiert vorliegen musste. Anhand der spektroskopischen Daten wurde darauf geschlossen, dass 2-Phenylethanol (91) isoliert wurde.

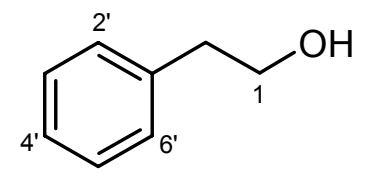

91

Die farblose, rosenartig riechende Flüssigkeit 91 ist als Bestandteil von Rosen-, Orangeblüten-, Neroli-, Geranien- und Nelkenölen bekannt.

\subsection{Streptomyces flavogriseus ACT7651}

Der Stamm ACT7651 wurde aus litoralem Sediment, welches in der Nähe von Punta Arenas (Feuerland) gesammelt wurde, isoliert. Gemäß der 16S rRNA-Sequenzanalyse besteht eine 98 \%ige Homologie zu Streptomyces flavogriseus, was einer vergleichsweise großen Distanz zum nächsten bekannten Verwandten entspricht. Dies könnte auf das Vorliegen einer neuen Spezies hindeuten.

\subsubsection{2'-Desoxyuridin}

Der weiße Feststoff 92 fiel im Dünnschichtchromatogramm $\left(R_{f}=0.05, \mathrm{CH}_{2} \mathrm{Cl}_{2} / \mathrm{MeOH} 9: 1\right)$ durch UV-Löschung bei $254 \mathrm{~nm}$ sowie durch Blaufärbung beim Besprühen mit Anisaldehyd auf.

Verbindung 92 zeigte im EI-Massenspektrum das Ion höchster Masse bei $m / z=228$. Dem ${ }^{1} \mathrm{H}-\mathrm{NMR}-$ Spektrum waren Signale bei $\delta=7.97$ und $5.69 \mathrm{zu}$ entnehmen, welche benachbarten Protonen an $\mathrm{sp}^{2}$-hybridisierten Kohlenstoffatomen zugeschrieben werden konnten. Zusätzlich konnten drei Methingruppen bei $\delta=6.26,4.37$ und 3.92 sowie zwei Methylengruppen bei $\delta=3.74$ und 2.22 detektiert werden, die aufgrund ihrer chemischen Verschiebungen und anhand des charakteristischen Kopplungsmusters einer Deoxyriboseeinheit zugeordnet wurden. Durch eine Suche in AntiBase ${ }^{30}$ und anschließenden Vergleich der ${ }^{1} \mathrm{H}$ NMR-Daten mit Literaturwerten konnte 92 als 2'-Desoxyuridin (92) identifiziert werden. 
<smiles>O=c1ccn(C2CC(O)C(CO)O2)c(=O)[nH]1</smiles>

92<smiles>Cc1cn(C2CC(O)C(CO)O2)c(=O)[nH]c1=O</smiles>

93<smiles></smiles>

94

\subsubsection{2'-Desoxythymidin}

Verbindung 93 fiel im Dünnschichtchromatogramm $\left(R_{f}=0.07, \mathrm{CH}_{2} \mathrm{Cl}_{2} / \mathrm{MeOH}\right.$ 9:1 $)$ durch UV-Löschung bei $254 \mathrm{~nm}$ sowie durch Blaufärbung beim Besprühen mit Anisaldehyd auf und konnte in Form eines weißen Feststoffs isoliert werden.

Im Vergleich mit 92 fehlten im ${ }^{1}$ H-NMR-Spektrum von 93 die Signale für Methingruppen bei $\delta=7.97$ und 5.69, stattdessen traten Resonanzen einer zusätzlichen Methineinheit bei $\delta=7.80$ sowie einer Methylgruppe bei $\delta=1.86$ auf. Das ESI-Massenspektrum zeigte ein Ion bei $m / z=265[\mathrm{M}+\mathrm{Na}]^{+}$. Anhand dieser Befunde konnte 93 als $2^{\prime}$-Desoxythymidin (93) identifiziert werden. Ein Vergleich der NMR-Daten aus der Spektrensammlung bestätigte die Zuordnung.

\subsubsection{2'-O-Methyluridin}

Die sich im Dünnschichtchromatogramm mit Anisaldehyd nach Erhitzen grün anfärbende, UV-Licht bei $254 \mathrm{~nm}$ löschende Verbindung 94 zeigte im ESI-Massenspektrum ein Ion bei $m / z=259[\mathrm{M}+\mathrm{H}]^{+}$. Ein Vergleich des ${ }^{1} \mathrm{H}-\mathrm{NMR}-$ Spektrums mit demjenigen von Verbindung 92 ließ auf das Vorliegen desselben Uracil-Grundgerüsts schließen, jedoch fehlte das Signal einer Methylengruppe bei $\delta=2.22$. An deren Stelle traten zwei zusätzliche Resonanzen, die einer Methingruppe $(\delta=3.87)$ und einer Methoxygruppe $(\delta=3.51)$ zugeteilt wurden. Somit konnte 66 als 2'-O-Methyluridin (94) identifiziert werden, was durch eine Suche mit AntiBase ${ }^{30}$ und Vergleich der NMR-Daten mit Literaturwerten ${ }^{109}$ bestätigte wurde. 2'-O-methylierte Ribonucleoside werden bei der ribosomalen RNA-Synthese eingebaut. Vermutlich dienen sie dazu, benachbarte Phosphodiesterbindungen vor einem Abbau durch intrazelluläre RNasen zu schützen. 


\subsubsection{Enterocin}

Im Dünnschichtchromatogramm war 95 als bei $254 \mathrm{~nm}$ UV-löschende, sich mit Anisaldehyd in der Wärme blau anfärbende Zone erkennbar. Im ${ }^{1} \mathrm{H}-\mathrm{NMR}-$ Spektrum konnten charakteristische Signale für einen monosubstituierten Phenylring bei $\delta=7.84(\mathrm{~d}, 2 \mathrm{H}), 7.51$ (t, $1 \mathrm{H})$ und $7.39(\mathrm{t}, 2 \mathrm{H})$ sowie für Protonen eines konjugierten Doppelbindungssystems bei $\delta=6.30\left(\mathrm{~d},{ }^{4} \mathrm{~J}=2.2 \mathrm{~Hz}\right), 5.42\left(\mathrm{~d},{ }^{4} J=2.2 \mathrm{~Hz}\right)$ detektiert werden. Zusätzliche waren Resonanzen für eine Methoxygruppe bei $\delta=3.75$, vier Methingruppen bei $\delta=4.77,4.64,4.61$ und 4.42 sowie eine diastereotope Methylengruppe bei 2.53 und 1.84 vorhanden. Das ESIMassenspektrum zeigte ein Ion bei $m / z=445[\mathrm{M}+\mathrm{H}]^{+}$. Die Molmasse führte in Kombination mit den abgeleiteten Strukturfragmente in einer AntiBase-Suche ${ }^{30}$ zur Verbindung Enterocin (95). Diese Substanz war bereits mehrfach in unserer Arbeitsgruppe isoliert worden. Ein Vergleich der NMR-Spektren bestätigte die Zuordnung.

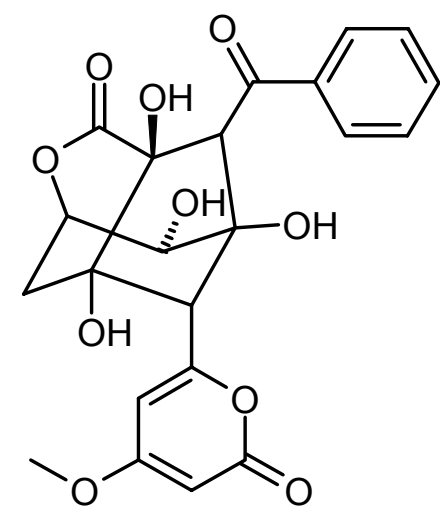

95

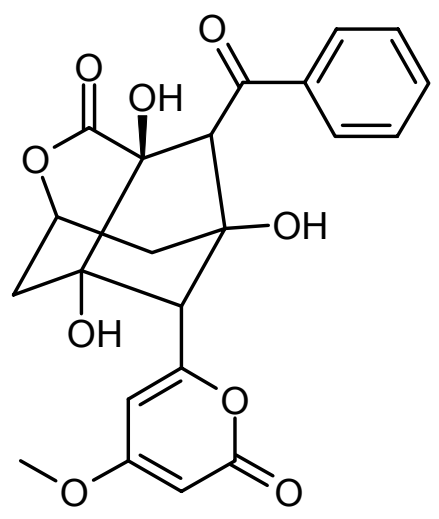

96

\subsubsection{5-Desoxyenterocin}

Die bei $254 \mathrm{~nm}$ UV-löschende Verbindung 96 färbte sich mit Anisaldehyd nicht an. Der farblose Feststoff zeigte im ${ }^{1} \mathrm{H}-\mathrm{NMR}-$ Spektrum identische Signale im Aromaten- und Doppelbindungsbereich wie Substanz 95. Im Unterschied hierzu fehlte jedoch die Resonanz einer Methingruppe, an deren Stelle Signale einer zusätzlichen diastereotopen Methylengruppe bei $\delta=2.83$ und 2.71 traten. Die übrigen Protonen zeichneten sich im Vergleich zu Verbindung 95 durch geringe Unterschiede in ihren chemischen Verschiebungen aus. Das ESI-Massenspektrum zeigte ein Ion bei $m / z=429[\mathrm{M}+\mathrm{H}]^{+}$, was einer Molmassendifferenz von 16 bezogen auf 95 entsprach. Dies legte die Vermutung einer fehlenden 
Hydroxygruppe nahe. Eine Suche in AntiBase $^{30}$ mit diesen Informationen führte zu 5Desoxyenterocin (96).

Enterocin (95), das auch unter dem Namen Vulgamycin bekannt ist, konnte inklusive mehrerer Derivate bereits aus dem marinen Actinomyceten Streptomyces marinus, ${ }^{110,111}$ aus terrestrischen Streptomyceten sowie aus einer marinen Tunikate isoliert werden. ${ }^{112}$ Alle Verbindungen zeigen antibakterielle, antifungische sowie herbizide Aktivität. ${ }^{113}$

Die Biosynthese von 95 und 96 verläuft über einen PKS II-katalysierten Mechanismus ausgehend von Benzoyl-SCoA (97). Diese ungewöhnliche Startereinheit wird durch oxidativen Abbau aus Phenylalanin gebildet (siehe Abbildung 56). ${ }^{114}$ Die dabei als Zwischenstufe vorkommende Zimtsäure konnte im Rahmen dieser Arbeit in Form des Säureamids 99 ebenfalls isoliert werden. Verbindung 97 wird über sieben Kettenverlängerungsschritte mit Malonyl-SCoA in das lineare Octaketid 98 überführt, aus welchem mittels dreier enzymkatalysierter Reaktionen unter Beteiligung einer Favorskii-Umlagerung Enterocin (95) hervorgeht. ${ }^{115}$ Das als Vorstufe auftretende 5-Desoxyenterocin (96) konnte folglich auch als Sekundärmetabolit des Stammes ACT2298 gefunden werden. Bei dem für die Produktion von 95 verantwortlichen Biosynthesegencluster handelt es sich um das erste bei marinen Organismen nachgewiesene Beispiel einer Polyketidsynthase vom Typ II. ${ }^{116,117}$ 
<smiles>CC(C)C(C)C(C)(C)C(=O)O</smiles>

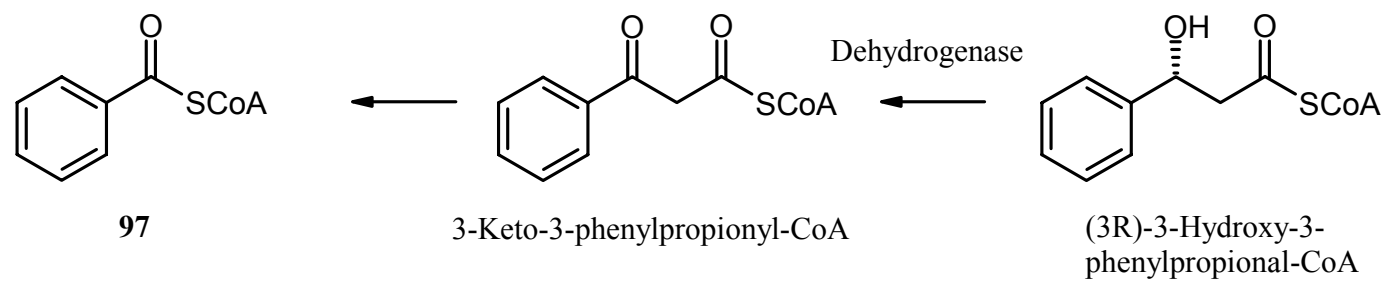
EncABCDL $\downarrow$ (x7 Malonyl-CoA)

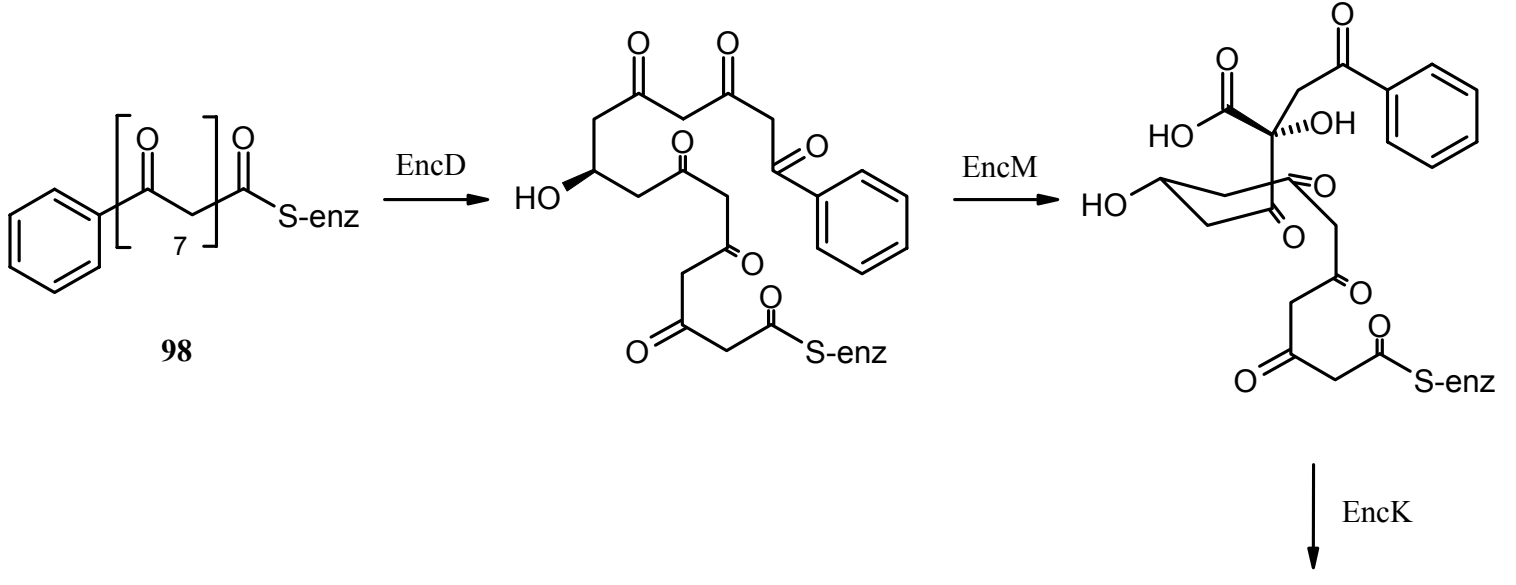

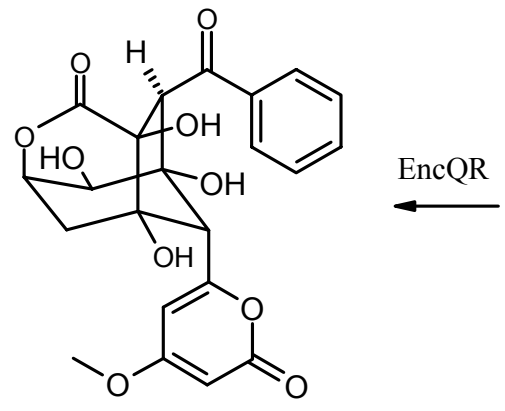

Enterocin<smiles>COc1cc(C2C[C@@H]3C(C(=O)c4ccccc4)CC4CC(O)(O2)C3(O)O4)oc(=O)c1</smiles>

5-Deoxyenterocin<smiles>COc1cc(C2OC3[C@H](C(=O)c4ccccc4)C4(O)CC(COC4=O)CC3(O)O2)oc(=O)c1</smiles>

3-epi-5-Deoxyenterocin

Abbildung 56 Biosyntheseweg für die Bildung von Enterocin und davon abgeleiteten Derivaten. $^{118}$ EncP: Desaminase Phenylalanin-Ammoniak-Lyase, EncH: Zimtsäure-CoA-Ligase, EncI: Zimtsäure-CoA-Hydratase, EncJ: 3-Keto-3phenylpropionyl-CoA-Thiolase, EncABCD: minimale PKS, EncL: aromatische Akyltransferase, EncM: Oxygenase, EncK: Methyltransferase, EncQ: Ferredoxin, EncR: Cytochrom-P-450-Hydroxylase. 


\subsection{6 trans-Zimtsäureamid}

Im Rohextrakt fiel 99 durch UV-Löschung bei $254 \mathrm{~nm}$ und hellrote Farbreaktion beim Besprühen mit Anisaldehyd sowie Gelbfärbung mit Ehrlichs Reagenz auf.

Im ${ }^{1} \mathrm{H}-\mathrm{NMR}-$ Spektrum waren zwei Dubletts bei $\delta=7.57$ und 6.74 detektierbar, die aufgrund ihrer chemischen Verschiebung und der zugehörigen Kopplungskonstante $\left({ }^{3} J=15.7\right.$ $\mathrm{Hz}$ ) zu einer trans-ständigen Doppelbindung gehören mussten. Weiterhin waren Signale für einen monosubstituierten Phenylring zu erkennen. Das EI-Massenspektrum zeigte ein Ion höchster Masse bei $m / z=146$ und das Fragmentierungsmuster für Zimtsäureamid (99). Ein Vergleich der NMR-spektroskopischen Daten bestätigte diesen Befund.<smiles>NC(=O)/C=C/c1ccccc1</smiles>

99

\subsubsection{Venturicidin A}

Die nicht von XAD-16 adsorbierte wässrige Phase lieferte nach Extraktion mit Essigester und chromatographischer Trennung farblosen Feststoff. Verbindung 100 fiel im Dünnschichtchromatogramm durch schwache UV-Löschung bei $254 \mathrm{~nm}$ und intensive dunkelblaue Färbung beim Besprühen mit Anisaldehyd und Ehrlichs Reagenz auf.

Die Substanz zeigte im ${ }^{1}$ H-NMR-Spektrum ein komplexes Signalmuster im Bereich $\delta=5.58-0.82$, während dem ${ }^{13} \mathrm{C}$-NMR-Spektrum die Existenz von 41 Kohlenstoffatomen im Molekül entnommen werden konnte. Im ESI-Massenspektrum war ein Ion bei $m / z=772[\mathrm{M}+\mathrm{Na}]^{+}$erkennbar. Diese Informationen deuteten auf das Vorliegen eines Makrolid-Grundgerüsts hin. Eine Datenbanksuche in AntiBase ${ }^{30}$ ergab, dass das aus einem 20-gliedrigen Lactonring aufgebaute, mit einem Zucker und einer $\mathrm{C}_{11}$-Alkyl-Seitenkette substituierte Antibiotikum Venturicidin A (100) isoliert wurde. Ein Vergleich der NMRDaten mit Literaturwerten bestätigte die Zuordnung. ${ }^{119}$ 


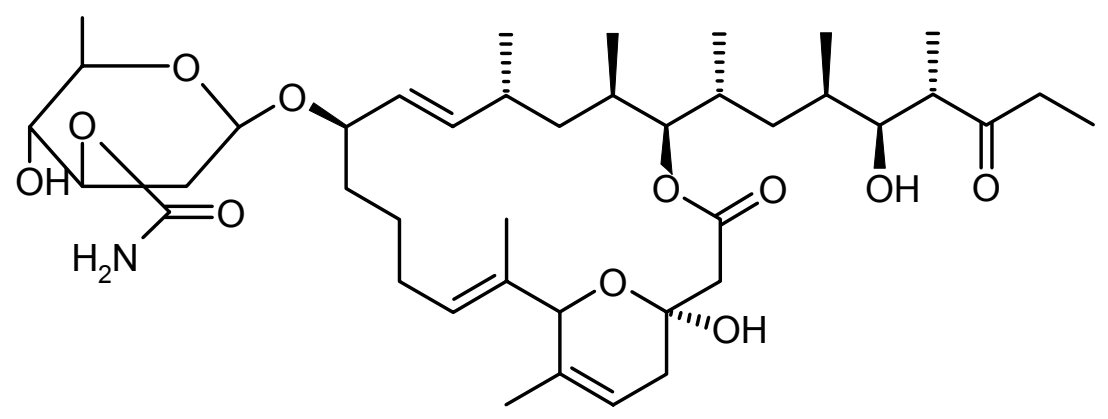

100

Substanz 100 wurde zum ersten Mal aus Kulturen des Stammes Streptomyces aureofaciens isoliert. ${ }^{120}$ Bei diesem antifungischen Makrolid handelt es sich ebenso wie bei der strukturell nahe verwandten Verbindung X-14952B (106) um ein Glykosid der 3-O-Carbamoyl2-desoxy-D-rhamnose. 


\section{Untersuchungen ausgewählter Stämme aus anderen Habitaten}

\subsection{Streptomyces sp. GW24/1811}

Der nach dreitägiger Kultivierung erhaltene Rohextrakt des aus Malta stammenden terrestrischen Stammes Streptomyces sp. GW24/1811 wies im Dünnschichtchromatogramm eine Reihe farbloser Zonen auf. Die Isolierung der entsprechenden Reinsubstanzen gelang aufgrund zu geringer Substanzmengen jedoch nicht. In einem zweiten, sechstätigen Kultivierungsversuch zeigte der erhaltene Rohextrakt im Dünnschichtchromatogramm schließlich mehrere orange und violette Zonen, welche sich bei Behandlung mit $2 \mathrm{~N} \mathrm{NaOH}$ blau bzw. braun verfärbten. Im biologischen Screening zeigte der Rohextrakt hohe Aktivitäten gegen Chlorella vulgaris, Nematoden sowie Salinenkrebse.

\subsubsection{Hopen-B}

Die farblose kristalline Verbindung 101 gab sich im Dünnschichtchromatogramm nach Anfärbung mit Anisaldehyd als blaue Zone zu erkennen. Sie zeigte weder bei $254 \mathrm{~nm}$ noch $366 \mathrm{~nm}$ UV-Aktivität und zeichnete sich durch geringe Polarität aus, welche sich in einem $R_{f}$-Wert von 0.98 bei Verwendung von $\mathrm{CH}_{2} \mathrm{Cl}_{2} / \mathrm{MeOH}$ 95:5 als Laufmittel widerspiegelt.

Das ${ }^{1}$ H-NMR-Spektrum wies starke Signalüberlappung im aliphatischen Bereich zwischen $\delta=0.7$ bis 1.8 auf. Darüber hinaus konnten Resonanzen für eine Methingruppe bei $\delta=2.66$ sowie eine Methylengruppe im Doppelbindungsbereich bei $\delta=4.76$ detektiert werden. Im APT-NMR-Spektrum wurden 30 Signale angezeigt, die sechs Methylgruppen, sechs Methingruppen sowie achtzehn Methylen- bzw. quartären Kohlenstoffatomen zugeordnet werden konnten. Darunter befanden sich Resonanzen eines quartären Kohlenstoffatoms bei $\delta=148.8$ sowie einer Methylengruppierung bei $\delta=110.1$, welche aufgrund ihrer chemischen Verschiebung $\mathrm{sp}^{2}$-hybridisiert vorliegen mussten. Im EI-Massenspektrum war das Ion höchster Masse bei $m / z=410$ erkennbar. Eine Suche in AntiBase ${ }^{30}$ lieferte Hopen B (101) als Strukturvorschlag. Ein Vergleich der spektroskopischen Daten den Literaturwerten bestätigte die Zuordnung. ${ }^{121}$ 


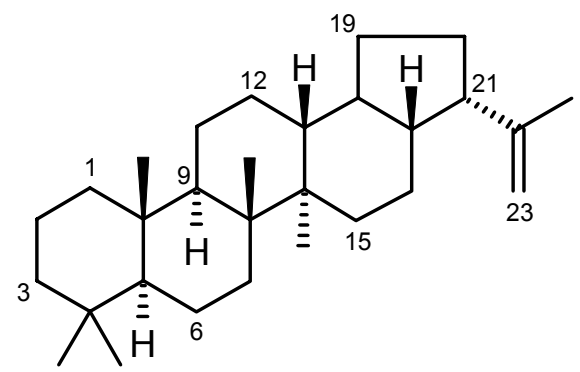

101

Bei den Triterpenen handelt es sich um eine Gruppe von Naturstoffen mit hoher struktureller wie funktioneller Diversität. Sie sind in die große Klasse der Isoprenoide eingegliedert, wobei ihre Struktur auf einem $\mathrm{C}_{30}$-Grundgerüst basiert. Von ihnen abgeleitet sind die tetracyclisch aufgebauten Sterole, welche in fast allen eukaryotischen, jedoch sehr selten in prokaryotischen Zellen biosynthetisiert werden. Mit Ausnahme der Archae ${ }^{122}$ findet man bei zahlreichen Bakterienarten stattdessen pentacyclische Triterpenoide, die der HopanReihe zuzuordnen sind. ${ }^{123,124}$ Diese für den Primärmetabolismus bedeutenden Verbindungen stabilisieren die Phospholipid-Doppelschichten in den Plasmamembranen. Bei Bradyrhizobien, einer an Wurzeln von Hülsenfruchtpflanzen symbiotisch lebenden Bakterien$\operatorname{art}^{125}$, besitzen Hopanoide eine zusätzliche Funktion. Hier werden sie zum Aufbau einer Diffusionsbarriere genutzt, welche den Schutz des oxidationsempfindlichen NitrogenaseKomplexes vor Sauerstoff sicherstellt. ${ }^{126}$

\subsubsection{Poly(B-hydroxybuttersäure)}

Der weiße polymerartige Feststoff $\mathbf{1 0 2}$ konnte im Dünnschichtchromatogramm weder durch UV-Licht noch mittels Sprühreagenzien sichtbar gemacht werden. Die Substanz löste sich gut in Dichlormethan sowie Chloroform, nicht jedoch in Methanol und Aceton.

Das ${ }^{1}$ H-NMR-Spektrum zeigte Signale für eine Methylgruppe bei $\delta=1.24$, eine diastereotope Methylengruppe bei $\delta=2.59$ und 2.44 sowie eine Methingruppe bei $\delta=5.23$. Letztere musste aufgrund der chemischen Verschiebung an ein Heteroatom gebunden sein. $\operatorname{Im}{ }^{13} \mathrm{C}$ NMR-Spektrum konnte zusätzlich eine Carbonylgruppe bei $\delta=169.1$ detektiert werden. Ein Vergleich der NMR-Spektren mit denjenigen von Poly(ß-hydroxybuttersäure) (102), eines in unserem Arbeitskreis häufig isolierten Polymers, führte zur Identifizierung der Verbindung. Das ESI-Massenspektrum wies keine signifikanten Ionen im Bereich bis $m / z=2000$ auf, was auf das Vorliegen langkettiger Poly(ß-hydroxybuttersäure)-Moleküle hindeutete. 


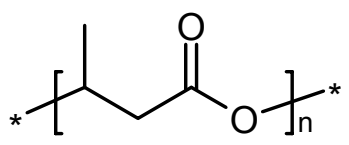

102

Polyhydroxysäuren stellen neben Polyisoprenoiden, Polypeptiden, Polysacchariden und Polynucleotiden eine weitere Gruppe weit verbreiteter Biopolymere dar. P(3-HB) (102) wurde erstmals 1925 von Lemoigne beschrieben, ${ }^{127}$ der dieses Polymer zu einem späteren Zeitpunkt aus Bacillus megaterium isolieren konnte. ${ }^{128}$ Polyhydroxysäuren werden von Mikroorganismen bei Nährstoffmangel in Gegenwart von Kohlenstoff-Quellen unter Energieverbrauch gebildet. ${ }^{129}$ Infolge ihres hohen Molekulargewichts und der damit einhergehenden Schwerlöslichkeit erzeugen Polyhydroxysäuren keinen osmotischen Druck, was zu ihrer Nutzung als mikrobielle Reservestoffe geführt hat. ${ }^{130}$ Neben diesen als sPHB (s für „storage“) bezeichneten Biopolymeren wurden in neuerer Zeit Vertreter einer als cPHB (c für „channel-forming“) beschriebenen Gruppe gefunden, welche sich durch geringe Molekulargewichte $(\mathrm{MW} \approx 1300 \mathrm{Da})$ auszeichnen. ${ }^{131}$

\subsubsection{Komodochinon A}

Verbindung 103 gab sich im Dünnschichtchromatogramm $\left(R_{f}=0.42, \mathrm{CH}_{2} \mathrm{Cl}_{2} / \mathrm{MeOH}\right.$ 95:5) durch eine orange Eigenfarbe, UV-Löschung bei $254 \mathrm{~nm}$ sowie Fluoreszenz bei $366 \mathrm{~nm}$ zu erkennen. Eine Farbreaktion beim Besprühen mit Anisaldehyd blieb aus.

Das ESI-Massenspektrum von 103 zeigte ein Ion bei $m / z=530[\mathrm{M}+\mathrm{H}]^{+}$, was auf eine ungerade Anzahl von Stickstoffatomen im Molekül schließen ließ. Im ${ }^{1}$ H-NMR-Spektrum war ein mit $\mathrm{D}_{2} \mathrm{O}$ austauschbares Signal $\delta=8.40$ erkennbar, welches für zwei Protonen integrierte. Weiterhin fanden sich Resonanzen für drei aromatische Methingruppen bei $\delta=7.95,7.84$ und 7.74, sechs weitere Methingruppen bei $\delta=5.78,4.97,4.18,3.98,3.83$ und 3.23, zwei diastereotope Methylengruppen bei $\delta=2.97,2.57,2.02$ und 1.83 sowie vier homotope Methylengruppen bei $\delta=2.57,2.58,1.28$ und 1.04. Aufgrund der geringen

Substanzmenge war die Messung eines ${ }^{13} \mathrm{C}$-NMR-Spektrums nicht möglich. Eine Suche in AntiBase $^{30}$ mit den beschriebenen Informationen lieferte Komodochinon A (103) als Strukturvorschlag. Ein Vergleich der NMR-Daten ergab jedoch keine zufriedenstellende Übereinstimmung, wobei Unterschiede vor allem im Bereich der chemischen Verschiebung der Zuckerprotonen lagen. Ein $\mathrm{MS}^{\mathrm{n}}$-Experiment zeigte allerdings, dass die 
Summenformel der Zuckerreste identisch war. Dies deutete darauf hin, dass dasselbe Aglykon- und Zuckergerüst wie bei Verbindung 103 vorlag, diese jedoch an anderer Position miteinander verknüpft waren.

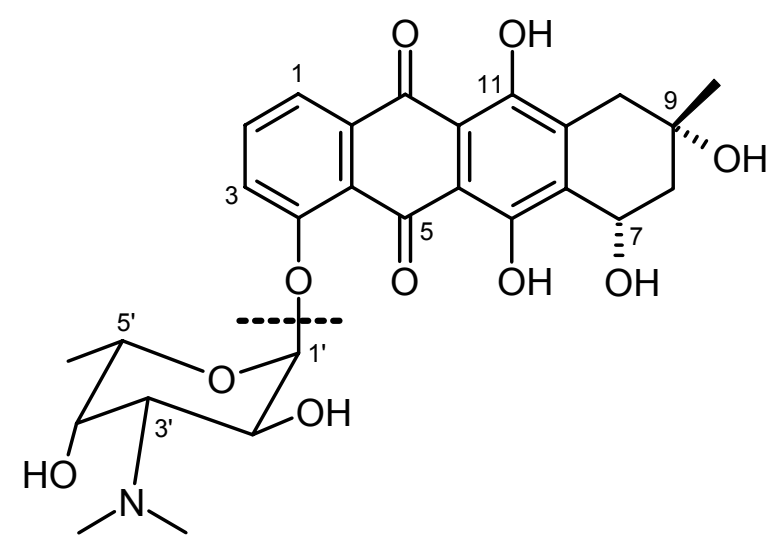

103

Komodochinon A (103) besteht aus einem Anthracyclin-Gerüst, welches mit einem Aminozucker am D-Ring substituiert ist. Untersuchungen ergaben, dass $\mathbf{1 0 3}$ die Differenzierung neuronaler Zellen induziert kann. ${ }^{132}$

\subsubsection{Komodochinon B}

Der orange Feststoff 104 wies identisches UV- und Anfärbeverhalten, jedoch geringere Polarität $\left(R_{f}=0.42, \mathrm{CH}_{2} \mathrm{Cl}_{2} / \mathrm{MeOH} 95: 5\right)$ als Verbindung 103 auf.

Das ESI-Massenspektrum von 104 zeigte ein Ion bei $m / z=379[\mathrm{M}+\mathrm{Na}]^{+}$. Im ${ }^{1} \mathrm{H}-\mathrm{NMR}-$ Spektrum waren drei mit $\mathrm{D}_{2} \mathrm{O}$ austauschbare Signale bei $\delta=13.40,12.06$ und 5.28 erkennbar. Darüber hinaus waren Resonanzen für drei aromatischen Protonen bei $\delta=7.81,7.75$ und 7.37, die einem 1,2,3-trisubstituierten Phenylring zugeschrieben wurden, eine Methingruppe bei $\delta=4.94$, zwei diastereotope Methylengruppen bei $\delta=3.02,2.53,2.05$ und 1.84 sowie eine Methylgruppe bei $\delta=1.32$ detektierbar. Dem ${ }^{13} \mathrm{C}-\mathrm{NMR}$-Spektrum waren neunzehn Signale zu entnehmen, von denen zwei im Bereich von Carbonylkohlenstoffatomen $(\delta=189.7$ und 185.7) lagen. Mit den vorliegenden Daten wurde eine Suche in AntiBase $^{30}$ durchgeführt, welche Komodochinon B (104) als Strukturvorschlag lieferte. Ein Vergleich der spektroskopischen Daten mit Literaturwerten bestätigte diese Zuordnung. 
<smiles></smiles>

104

Komodochinon A (103) und dessen Aglykon Komodochinon B (104) konnten erstmals im Jahr 2003 aus einem marinen Streptomyceten isoliert werden. ${ }^{132}$

\subsection{Streptomyces sp. GW16/3128}

Agarplatten, Mycel und Kulturbrühe des terrestrischen Stammes Streptomyces sp. GW16/3128 waren tief schwarz gefärbt, nach Extraktion der Kulturbrühe mit Essigester ergab sich jedoch ein hellgelber Rohextrakt. Im Dünnschichtchromatogramm fiel eine Zone auf, die schwach UV-löschend war und sich mit Anisaldehyd schwarz anfärbte. Im biologischen Screening zeigte der Rohextrakt sehr hohe Aktivitäten gegen Chlorella vulgaris, Chlorella sorokiniana sowie Scenedesmus subspicatus.

Eine Nährmedienvariation wurde durchgeführt, da der Stamm in $\mathrm{M}_{2}$-Medium kein ausreichendes Wachstum aufwies. Es zeigte sich, dass die Verwendung eines FleischextraktMediums hier deutliche Vorteile brachte. Zur Animpfung dienten flüssige Vorkulturen, da direkte Animpfung mittels gut bewachsener Agar-Stückchen $\mathrm{zu}$ keinem zufriedenstellenden Wachstum des Stammes führte.

\subsubsection{Glykolipide}

Der farblose Feststoff $\mathbf{1 0 5}$ zeigte im Dünnschichtchromatogramm sehr schwache UVLöschung und färbte sich mit Anisaldehyd schwarz an. Verbindung 105 konnte in einer Ausbeute von $0.3 \mathrm{mg} / \mathrm{l}$ isoliert werden.

Im ESI-Massenspektrum trat das Ion höchster Intensität bei $m / z=779[\mathrm{M}+\mathrm{Na}]^{+}$auf. Das ${ }^{1} \mathrm{H}-\mathrm{NMR}-$ Spektrum zeigte Signale für acht Methingruppen bei $\delta=5.33,5.24,4.79,3.60$, 3.56, 3.38 und 3.30 sowie drei diastereotope Methylengruppen bei $\delta=4.47,4.20,3.86$, 3.78, 3.66 und 3.62. Im Bereich von $\delta=2.4-0.9$ waren zusätzlich Resonanzen für Acylreste zu erkennen, welche unter Einbeziehung der Integralhöhen auf das Vorliegen zweier 
getrennter Ketten hindeuteten. Darüber hinaus war ein Signal bei $\delta=2.02$ detektierbar, welches aufgrund seiner chemischen Verschiebung und Intensität zwei an ein Doppelbindungssystem gebundenen Methylengruppen zugeschrieben werden konnten. Im ${ }^{13} \mathrm{C}$-NMRSpektrum war die genaue Anzahl der Kohlenstoffsignale schwer zu ermitteln, da im Bereich der Methylenresonanzen eine starke Signalüberlappung zu beobachten war. Diese konnte jedoch mittels einer ESI-Massenhochauflösung bestimmt werden, aus der sich eine Summenformel von $\mathrm{C}_{43} \mathrm{H}_{80} \mathrm{O}_{10}$ ergab. Die Auswertung der NMR-Daten legte die Vermutung nahe, dass Verbindung 105 aus einem Zuckergerüst und zwei Acylketten aufgebaut ist. Mit Hilfe von 2D-NMR-Spektren konnten die erhaltenen Strukturfragmente verknüpft und die Vermutung bestätigt werden. Die genaue Position der Doppelbindung in einer der Acyl-Seitenketten des Glycolipids 105 konnte jedoch nicht eindeutig bestimmt werden.

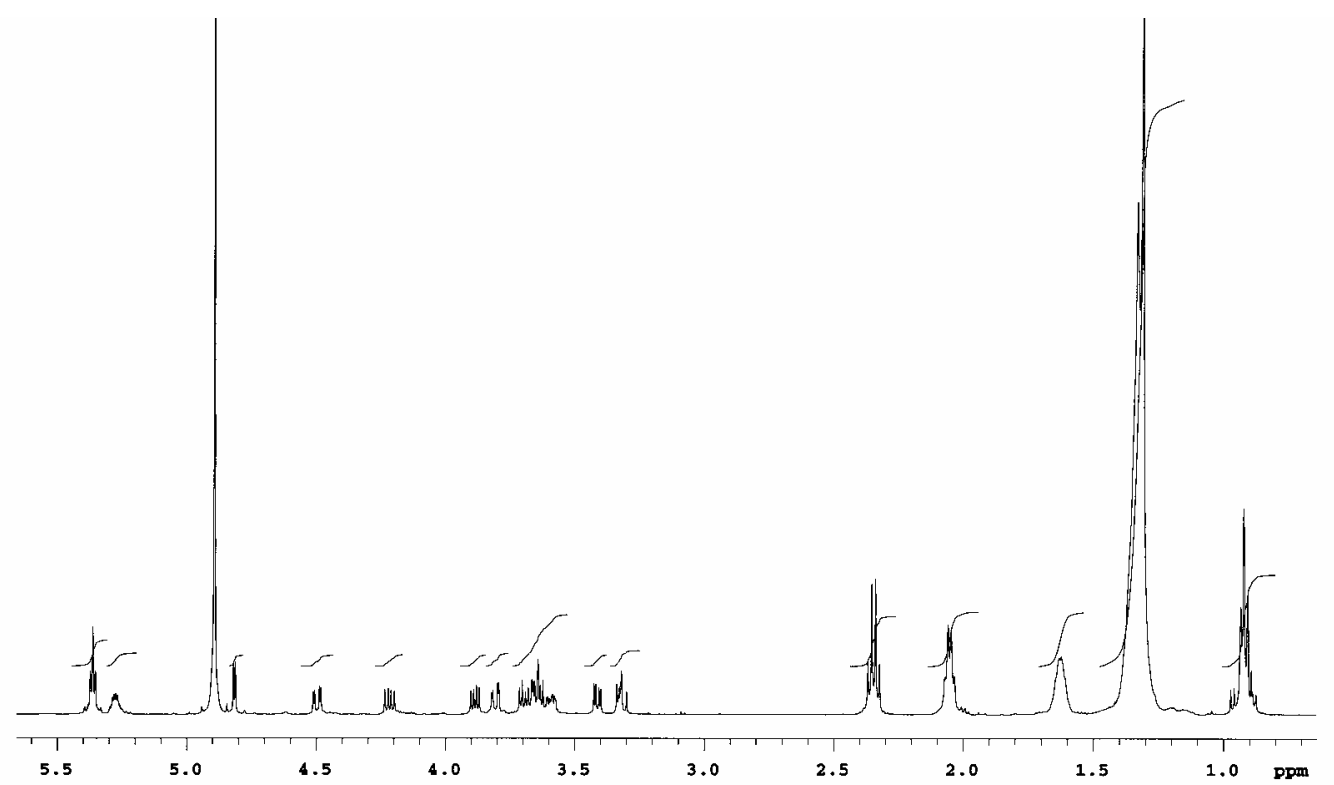

Abbildung $57{ }^{1} \mathrm{H}-\mathrm{NMR}-\mathrm{Spektrum}\left(600 \mathrm{MHz}, \mathrm{CD}_{3} \mathrm{OD}\right)$ von 105.

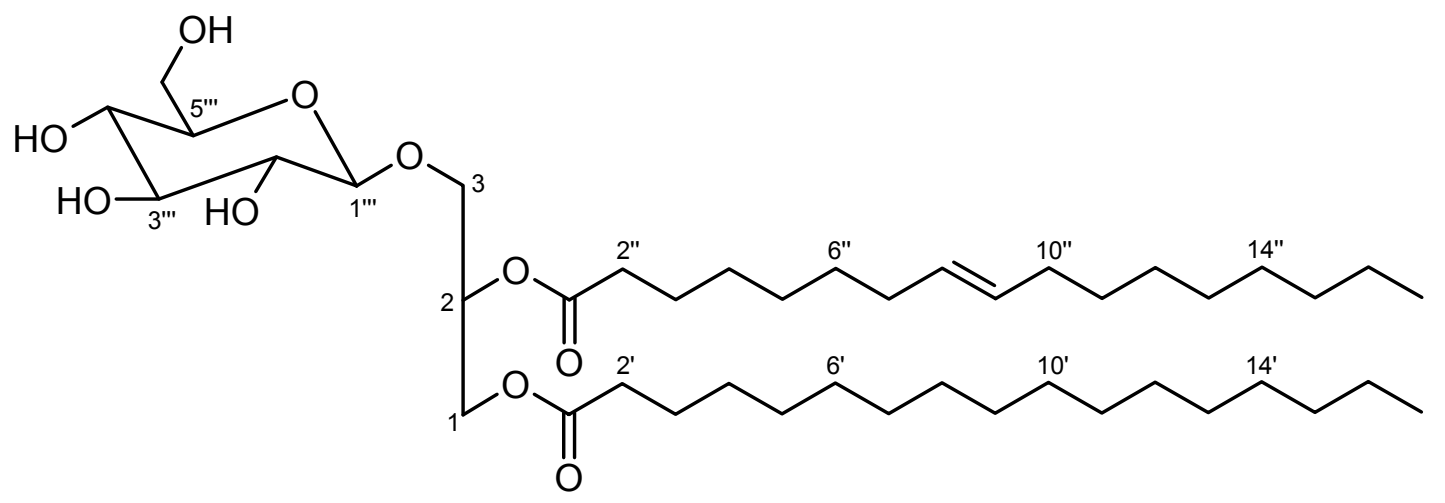


Glykolipide sind bei photosynthetisch aktiven Eukaryoten ${ }^{133}$ und Prokaryoten ${ }^{134}$ weit verbreitet. In biologischen Untersuchungen zeigten Glykolipide antitumorale, ${ }^{135}$ entzündungshemmende, ${ }^{136}$ phycotoxische,${ }^{137}$ hemolytische ${ }^{138}$ sowie antivirale ${ }^{139}$ Aktivität.

\subsection{Streptomyces sp. GW12/1492}

Der intensiv gelb gefärbte Rohextrakt des terrestrischen Stammes Streptomyces sp. GW12/1492 zeigte im Dünnschichtchromatogramm zwei auffällige Zonen. Die unpolarere Zone besaß eine gelbe Eigenfarbe und färbte sich mit herkömmlichen Sprühreagenzien nicht an, während die polarere Zone farblos war und beim Besprühen mit Anisaldehyd sowie Ehrlichs Reagenz eine dunkelblaue Farbreaktion einging. Darüber hinaus konnte im Dünnschichtchromatogramm auf das Vorhandensein einer großen Menge an Fettsäuren geschlossen werden, welche sich durch nicht UV-löschende, mit Anisaldehyd blau anfärbbare Zonen zu erkennen gaben. Im biologischen Screening zeigte der Rohextrakt antibakterielle Aktivität.

\subsubsection{X-14952B}

Der Hauptmetabolit 106 des Stammes GW 12/1492 wurde in einer Ausbeute von

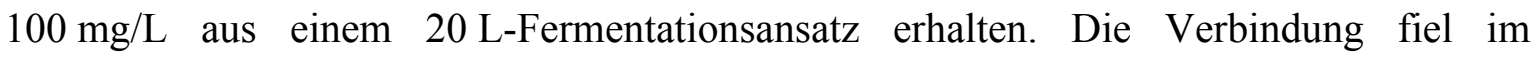
Dünnschichtchromatogramm $\left(R_{f}=0.28, \mathrm{CH}_{2} \mathrm{Cl}_{2} / \mathrm{MeOH}\right.$ 95:5) durch UV-Löschung bei $254 \mathrm{~nm}$ sowie durch intensive dunkelgrüne Färbung beim Besprühen mit Anisaldehyd auf. Der hellgelbe Feststoff 106 zeigte im ESI-Massenspektrum ein Ion bei $m / z=802[\mathrm{M}+\mathrm{Na}]^{+}$. Das ${ }^{1}$ H-NMR-Spektrum wies ein sehr komplexes Signalmuster auf, während im ${ }^{13} \mathrm{C}$-NMRSpektrum die Resonanzen von 42 Kohlenstoffatomen sichtbar waren. Anhand dieser Informationen wurde das Vorliegen eines Makrolid-Grundgerüsts vermutet. Eine Datenbanksuche in AntiBase ${ }^{30}$ ergab, dass das 20-gliedrige Makrolidlacton X-14952B (106) isoliert wurde. Ein Vergleich der NMR-Daten mit Literaturwerten bestätigte die Zuordnung. ${ }^{140}$ 


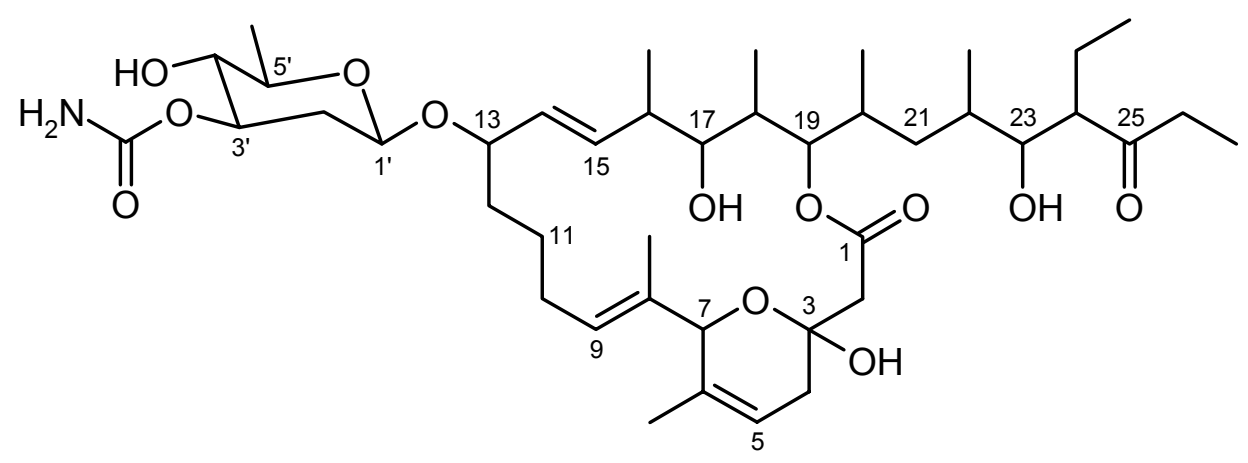

106

Verbindung 106 wurde erstmals 1985 von OMURA et al. als Sekundärstoff eines Streptomyceten-Stammes isoliert. Die Substanz besitzt antibakterielle und antifungische Aktivität. Ähnliche Wirkungen konnten auch bei den strukturell verwandten Makroliden Concanamycin, ${ }^{141}$ Virustomycin $\mathrm{A}^{142}$ und Bafilomycin ${ }^{143}$ gefunden werden.

\subsubsection{Cervinomycin $\mathbf{A}_{2}$}

Verbindung 107 wurde als Hauptkomponente einer Mischung unterschiedlicher gelber Substanzen, welche in einer Ausbeute von insgesamt $50 \mathrm{mg} / \mathrm{L}$ erhalten wurden, isoliert. Die Auftrennung der übrigen Substanzen war aufgrund der geringen Löslichkeit nicht möglich. Verbindung 107 zeigte UV-Löschung bei $254 \mathrm{~nm}$, war mit den üblichen Sprühreagenzien jedoch nicht anfärbbar.

Das ESI-Massenspektrum von 107 wies ein Ion bei $m / z=550[\mathrm{M}+\mathrm{Na}]^{+}$auf. Im ${ }^{1} \mathrm{H}-\mathrm{NMR}-$ Spektrum waren Signale für eine NH-/OH-Gruppe bei $\delta=13.96$, fünf aromatische Methingruppen bei $\delta=8.19,7.96,7.60,7.21$ und 7.14 sowie drei diastereotope Methylengruppen bei $\delta=4.25,4.04,3.74,3.32$ und 3.23 vorhanden. Weiterhin konnten Resonanzen für zwei Methoxygruppen bei $\delta=4.00$ sowie eine Methylgruppe bei $\delta=1.41$ detektiert werden. Dem ${ }^{13}$ C-NMR-Spektrum konnten 29 Kohlenstoffsignale entnommen werden. Eine Suche in AntiBase ${ }^{30}$ mit den vorliegenden Informationen lieferte Cervinomycin $\mathrm{A}_{2}$ (107) als Strukturvorschlag. Ein Vergleich der spektroskopischen Daten mit Literaturwerten bestätigte die Zuordnung. ${ }^{144}$ 


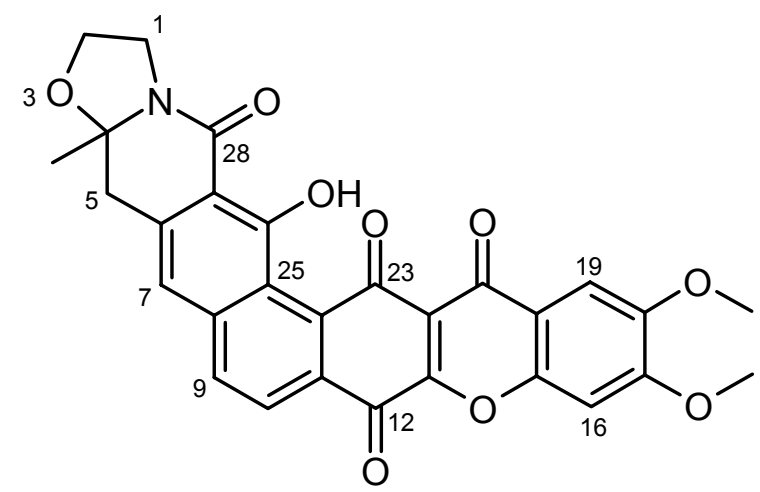

107

Die in allen herkömmlichen Lösungsmitteln schwerlöslichen Cervinomycine sind gegen Anaerober und Mycoplasmen wirksame Antibiotika, welche erstmals aus Kulturen von Streptomyces cervinus isoliert wurden. ${ }^{145}$ Für die medizinische Anwendung wird gegenwärtig die acetylierte Form von Verbindung 107 untersucht, welche sich durch bessere Löslichkeit und eine geringe Toxizität auszeichnet. Cervinomycin $\mathrm{A}_{2}$ (107) weist hohe Aktivität gegen die Problemkeime Clostridium difficile, Reptococcus variabillis und Streptococcus mutans auf. ${ }^{146}$

\subsubsection{Sonstige Metaboliten}

\section{Hexadecan- und Heptadecansäure}

Das zweite Hauptmetabolit 108 des Stammes GW 12/1492 fiel im Dünnschichtchromatogramm durch blaue Anfärbung mit Anisaldehyd auf. Substanz 108 konnte in Form eines farblosen Öls in einer Ausbeute von $100 \mathrm{mg} / \mathrm{L}$ isoliert werden. Anhand der ${ }^{1} \mathrm{H}-\mathrm{NMR}-$ und EI-Daten wurde die Verbindung 108 als eine Mischung aus Hexa- und Heptadecansäure (108) identifiziert.

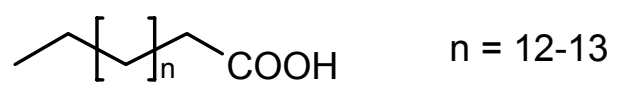

108

\section{Anthranilsäureamid und Phenylessigsäure}

Der farblose Feststoff 109 zeigte im Dünnschichtchromatogramm eine bei $254 \mathrm{~nm}$ UVlöschende sowie bei $366 \mathrm{~nm}$ hellblau fluoreszierende Zone, welche beim Besprühen mit 
Anisaldehyd bzw. Ehrlichs Reagenz Gelbfärbung einging. ${ }^{1} \mathrm{H}-\mathrm{NMR}$ - und EI-Massenspektrum bestätigten die Vermutung, dass es sich bei Verbindung 109 um Anthranilsäureamid (109) handelte.<smiles>NC(=O)c1ccccc1N</smiles>

109<smiles>O=C(O)Cc1ccccc1</smiles>

61

Der UV-löschende, farblose Feststoff 61 wies im ${ }^{1}$ H-NMR- und EI-Massenspektrum das für Phenylessigsäure (61) charakteristische Signalmuster auf. Substanz 61 war zuvor bereits als Metabolit des Actinomyceten-Stammes T421-A isoliert worden (siehe Kapitel 6.4.2).

\subsection{Streptomyces sp. GW51/456}

Eine chromatographische Aufreinigung der Fraktionen erwies sich als äußerst schwierig, da die zu trennenden Substanzen an Kieselgel wie an Sephadex adsorbiert blieben und auch durch Verwendung verschiedener Lösungsmittel und -gemische nicht eluiert werden konnten.

\subsubsection{Thiothymin}

Der weiße Feststoff 110 zeigte im Dünnschichtchromatogramm eine auffällige UV-Löschung bei $254 \mathrm{~nm}$.

Dem ${ }^{1}$ H-NMR-Spektrum konnten lediglich zwei Singuletts bei $\delta=7.84$ sowie 2.31 entnommen werden, welche einer Methin- bzw. einer Methylgruppe zugeordnet wurden. Das DCI-MS-Spektrum zeigte ein Ion bei $m / z=143[\mathrm{M}+\mathrm{H}]^{+}$. Anhand eine Suche in AntiBase ${ }^{30}$ und anschließendem Vergleich der ${ }^{1}$ H-NMR-Daten mit Literaturwerten konnte 110 als Thiothymin (110) identifiziert werden. 
<smiles>Cc1c[nH]c(=O)[nH]c1=S</smiles>

110

\subsubsection{B-Indomycinon}

Die orange Substanz 111 fiel im Dünnschichtchromatogramm $\left(R_{f}=0.75, \mathrm{CH}_{2} \mathrm{Cl}_{2} / \mathrm{MeOH}\right.$ 95:5) durch Eigenfarbe und UV-Löschung bei $254 \mathrm{~nm}$ auf. Die nach Behandlung mit Natronlauge einsetzende Blaufärbung legte die Vermutung nahe, dass es sich bei 111 um ein Chinon-Derivat mit chelierter Hydroxygruppe handelt.

Im ${ }^{1}$ H-NMR-Spektrum war anhand der Integralverhältnisse erkennbar, dass ein Gemisch aus zwei Substanzen vorliegen musste. Mittels eines anschließenden LC-MS-Experiments konnten zwei Isomere (111 und 112) mit einer Molmasse von 404 identifiziert werden, welche in den folgenden Abschnitten beschrieben werden.

Bei den ${ }^{1} \mathrm{H}-\mathrm{NMR}$-Signalen der Hauptkomponente handelte es sich um zwei mit $\mathrm{D}_{2} \mathrm{O}$ austauschbare Resonanzen bei $\delta=12.84$ und 2.76, zwei Singuletts bei $\delta=8.06$ und 6.51 sowie charakteristische Resonanzen für einen 1,2,3-trisubstituierten Aromaten bei $\delta=7.81,7.67$ und 7.34. Im aliphatischen Bereich waren zwei Methingruppen bei $\delta=5.70$ und 5.35, eine diastereotope Methylengruppen bei $\delta=2.90$ und 2.75 sowie drei Methylgruppen bei $\delta=1.68,1.62$ und $3.02 \mathrm{zu}$ erkennen. Eine Suche in AntiBase ${ }^{30}$ lieferte B-Indomycinon (111) als Strukturvorschlag für 111. Ein Vergleich des ${ }^{1}$ H-NMR-Daten mit einem in unserer Arbeitsgruppe vorhandenen Referenzspektrum bestätigte die Zuordnung. BIndomycinon (112) gehört zur Substanzklasse der Pluramycine. Diese verfügen über die Eigenschaft, selektiv an DNA binden zu können, worauf ihre antimikrobielle und antitumorale Aktivität zurückgeführt wird. ${ }^{147}$ 


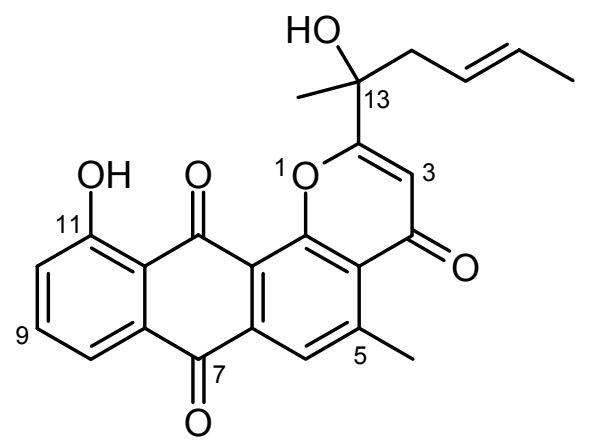

111

\subsubsection{Saptomycin A}

Ein Vergleich der ${ }^{1}$ H-NMR-Signale der Minderkomponente 112 mit denjenigen von 111 deutete auf das Vorliegen einer ähnlichen Struktur mit demselben Anthrachinon-Grundgerüst hin. Es fehlten jedoch im Unterschied zu 111 die Signale der Methylengruppe, stattdessen fanden sich zusätzliche Resonanzen für zwei Methingruppen bei $\delta=5.00$ und 2.97. Weiterhin war auffällig, dass sich die chemische Verschiebung der aliphatischen Doppelbindungsprotonen sowie der Methylgruppen im Vergleich zu 111 unterschied. Zusätzlich verfügte eine der Methylgruppe über ein verändertes Aufspaltungsmuster. Eine Suche in AntiBase ${ }^{30}$ nach einer Verbindung mit demselben Anthrachinon-Gerüst und der gleichen Molmasse wie 111 führte zu Saptomycin A (112). Ein Vergleich der ${ }^{1}$ H-NMRDaten von 112 mit einem Referenzspektrum bestätigte, dass es sich bei 112 um Saptomycin A handelt.<smiles>C/C=C/C(O)C(C)c1cc(=O)c2c(C)cc3c(c2o1)C(=O)c1c(O)cccc1C3=O</smiles>

112

Saptomycin A (112) wurde erstmals im Jahr 1993 von ABE et al. als Stoffwechselprodukt von Streptomyces sp. HP530 isoliert. Die Saptomycine verfügen über antimikrobiotische Eigenschaften und zeigen Antitumor-Aktivität gegen verschiedene Tumorzelllinien. ${ }^{148}$ 


\subsection{Streptomyces sp. Mei 24}

Der nach sechstägiger Kultivierung erhaltene Rohextrakt des Stammes Mei 24 zeichnete sich durch eine grünliche Färbung aus. Im Dünnschichtchromatogramm waren zahlreiche bei Tageslicht farbige Zonen sichtbar. Nach Besprühen mit Anisaldehyd zeigte sich eine ausgedehnte, blau angefärbte Zone, welche aufgrund fehlender UV-Löschung bei $254 \mathrm{~nm}$ auf das Vorliegen mehrerer Fettsäuren hindeutete. Nach deren Abtrennung verblieb lediglich eine sehr geringe Menge an Rohextrakt, aus der nur mühsam einige weitere Metaboliten isoliert werden konnten.

\subsubsection{4-Methylamino-7-methyl-isoquinolin-3,6-dion}

Der rote Feststoff 113 bildete im Dünnschichtchromatogramm $\left(R_{f}=0.70, \mathrm{CH}_{2} \mathrm{Cl}_{2} / \mathrm{MeOH}\right.$ 9:1) eine bei $254 \mathrm{~nm}$ UV-löschende, orange Zone, die sich beim Versetzen mit $2 \mathrm{~N} \mathrm{NaOH}$ rot verfärbte. Nach Besprühen mit Ehrlichs Reagenz stellte sich eine orange Färbung ein.

Im ESI-Massenspektrum von 113 konnte ein Ion bei $m / z=203[\mathrm{M}+\mathrm{H}]^{+}$detektiert werden. Dem ${ }^{1} \mathrm{H}-\mathrm{NMR}-$ Spektrum waren Signale für ein $\mathrm{OH}-\mathrm{NH}-$ Gruppe bei $\delta=5.79$, zwei aromatische Methingruppen bei $\delta=9.00$ und 5.70 sowie drei Methylgruppen bei $\delta=2.90,2.73$ und $2.68 \mathrm{zu}$ entnehmen. Eine Datenbanksuche in AntiBase ${ }^{30}$ führte zur Verbindung 4Methylamino-7-methyl-isoquinolin-3,6-dion (113), welche bereits mehrfach in unserem Arbeitskreis isoliert wurde. ${ }^{149,150}$ Ein Vergleich der spektroskopischen Daten bestätigte die Zuordnung. In der Literatur war 113 bislang lediglich als Syntheseprodukt, nicht jedoch als Naturstoff bekannt. ${ }^{151}$<smiles>CNC1=CC(=O)c2cc(C)ncc2C1=O</smiles>

113<smiles>CNC1=CC(=O)c2c(cnc(C)c2C)C1=O</smiles>

114

\subsubsection{4-Methylamino-7,8-dimethyl-isoquinolin-3,6-dion}

Die rote Verbindung 114 zeigte im Dünnschichtchromatogramm einen etwas unterschiedlichen $R_{f}$-Wert $\left(R_{f}=0.67, \mathrm{CH}_{2} \mathrm{Cl}_{2} / \mathrm{MeOH}\right.$ 9:1) im Vergleich mit 113. Beide Verbindungen besaßen jedoch identisches UV- und Anfärbeverhalten. 
Im ESI-Massenspektrum zeigte 114 ein Ion bei $m / z=217[\mathrm{M}+\mathrm{H}]^{+}$und wies somit eine um vierzehn Einheiten geringere Masse als 113 auf. Auch das ${ }^{1} \mathrm{H}-\mathrm{NMR}-\mathrm{Spektrum}$ fiel durch große Ähnlichkeit zu demjenigen von 113 auf. Es fehlte jedoch das Signal einer Methylgruppe, stattdessen trat die Resonanz einer zusätzlichen aromatischen Methingruppe bei $\delta=7.75$ auf. Letztere musste aufgrund der chemischen Verschiebung an das Kohlenstoff in Position 7 gebunden sein.<smiles>CC1=C(N)C(=O)c2c(ccnc2C)C1=O</smiles>

115

Das strukturell ähnlich aufgebaute Isochinolinchinon Cribrostatin 1 (115) konnte aus dem blauen marinen Schwamm Cribrochalina sp. isoliert werden. Verbindung 115 wies eine biologische Aktivität gegenüber der P388 lymphozytären Leukämie-Zelllinie auf. ${ }^{152}$

\subsubsection{Sonstige Metaboliten}

\section{3-Indolylcarbonsäure und 3-Hydroxyacetylindol}

Im Dünnschichtchromatogramm waren zwei UV-löschende Zonen auffällig, welche nach Besprühen mit Ehrlichs Reagenz eine violette Farbreaktion eingingen. Dies legte die Vermutung nahe, dass es sich hierbei um Indolderivate handelte. Ein Vergleich der EIMassen- und ${ }^{1} \mathrm{H}-\mathrm{NMR}-S p e k t r e n$ mit denjenigen der Verbindungen 16 und $\mathbf{1 7}$ ergab, dass 3-Indolylcarbonsäure (16) bzw. 3-Hydroxyacetylindol (17) isoliert wurde.

\section{Uracil und $p$-Hydroxybenzoesäure}

Die Substanzen 18 und 19 waren im Dünnschichtchromatogramm als UV-löschende Zonen im polaren Bereich erkennbar. Die nach Auftrennung erhaltenen farblosen Feststoffe konnten anhand ihrer spektroskopischen Eigenschaften rasch als Uracil (18) und $p$-Hydroxybenzoesäure (19) identifiziert werden. 


\section{$8 \quad$ Zusammenfassung}

Mikroorganismen nehmen einen großen Einfluss auf ökologische und symbiotische Wechselwirkungen in Lebensgemeinschaften, der durch Sekundärmetabolite mit oft einzigartiger Struktur und biologischer Aktivität moderiert wird. Bei den außergewöhnlichen Habitaten Arktis und Antarktis handelt es sich um in diesem Kontext bisher nur wenig untersuchte Ökosysteme. Um Grundlagen zu einem besseren Verständnis der ökologischen und symbiotischen Zusammenhänge zu liefern, über welche Lebensgemeinschaften bei derart unwirtlichen Umweltbedingungen interagieren, war die Untersuchung polarer mikrobieller Sekundärmetaboliten Thema der vorliegenden Arbeit.

Die zu untersuchenden polaren Bakterienstämme wurden vom Alfred-Wegener-Institut (Bremerhaven) zur Verfügung gestellt. Nach einem biologischen und chemischen Screening von mehr als 700 Rohextrakten wurden besonders auffällige Stämme ausgewählt (ca. $20 \%$ ), von denen anschließend eine Auswahl am Institut für Biotechnologie und Wirkstoffforschung (Kaiserslautern) im größeren Maßstab kultiviert wurde. Da die erhaltenen Mengen an Rohextrakten sehr gering und die Gemische sehr komplex zusammengesetzt waren, erwies sich deren chromatographische Aufarbeitung als recht aufwendig.

Um ein effizientes und zuverlässiges chemisches Screening zu ermöglichen, wurde zu Beginn der vorliegenden Arbeit die HPLC-UV-ESI-MS/MS-Kopplung als geeignete Methode für eine verlässliche Dereplikation weiterentwickelt, die mit dem Programm MSManager von ACDLabs eine schnelle und verlässliche Wiedererkennung bekannter Substanzen möglich machte.

Aufgrund der ungewissen Ergebnislage, welche die Bearbeitung polarer Bakterienstämme liefern würde, wurden zusätzlich terrestrische und marine Streptomyceten-Stämme, welche vom Institut für Bodenkunde (Lohra-Kirchvers) und der Fachhochschule Ostfriesland (Emden) bereitgestellt wurden, im biologischen und chemischen Screening untersucht. Fünf auffällige Stämme wurden in größerem Maßstab kultiviert und die gebildeten Sekundärstoffe isoliert.

\section{HPLC-UV-ESI-MS/MS-Datenbank und ESI-Fragmentierung}

Die Verwendung der HPLC-UV-ESI-MS/MS-Kopplung ermöglichte eine Trennung der in Rohextrakten bzw. Fraktionen erhaltenen Verbindungen bei gleichzeitiger Messung ver- 
schiedener stoffspezifischer Eigenschaften. Die hier experimentell zugänglichen Daten wie Retentionszeit, UV-Spektrum, Molmasse und Fragmentierungsmuster erwiesen sich in den meisten Fällen als ausreichend für eine eindeutige Identifizierung der untersuchten Substanzen. Zur Erstellung der Datenbank wurden bisher etwa 250 bakterielle Naturstoffe aus unserer Arbeitsgruppe herangezogen, wobei sämtliche massenspektrometrischen Daten sowohl im positiven als auch im negativen Modus detektiert wurden. Es zeigte sich jedoch, dass aufgrund der hohen Diversität an Naturstoffstrukturen und der messtechnischen Begrenzungen des Massenspektrometers nicht alle Verbindungen auswertbar waren. Als limitierende Faktoren erwiesen sich hier vor allem mangelnde ES-Ionisierbarkeit und/oder fehlende UV-Absorption.

Um erste strukturelle Rückschlüsse über Verbindungen erhalten zu können, welche neu bzw. noch nicht in der Datenbank implementiert sind, erwies sich die Aufklärung der charakteristischen, bisher nur wenig untersuchten ESI-Fragmentierungswege als vielversprechend. Dies konnte am Beispiel zweier oft isolierter Verbindungsklassen, den Actinomycinen $\left(\mathrm{Na}^{+}\right.$-Addukte) und Diketopiperazinen $\left(\mathrm{H}^{+}\right.$-Addukte), gezeigt werden. Bei den Actinomycinen war eine komplette Zuordnung der Aminosäure-Sequenzen auf der Basis der gemessenen MS/MS-Spektren möglich, was dieses Verfahren anderen Methoden überlegen macht.

\section{Arktische und Antarktische Bakterien}

Der aus dem Darminhalt der antarktischen Krillart Euphausia superba isolierte psychrotolerante Stamm Pseudoalteromonas sp. T268 produzierte Glutarsäure, Diketopiperazine und Fettsäuren als Hauptmetabolite. Neben Isatin (19) konnten auch einige seiner Derivate isoliert werden, nämlich 3-Hydroxy-3-acetonyloxindol (20) und 3-Hydroxy-3-(1propanonyl)oxindol (21). Verbindung 21 war bisher in der Literatur weder als Syntheseprodukt noch als Naturstoff beschrieben worden.<smiles>O=C1Nc2ccccc2C1=O</smiles>

19<smiles>[R]C(=O)CC1(O)C(=O)Nc2ccccc21</smiles>

$20 \mathrm{R}=\mathrm{CH}_{3}$

$21 \mathrm{R}=\mathrm{C}_{2} \mathrm{H}_{5}$ 
Als weitere Verbindungsklasse konnten Ubichinone aus dem Rohextrakt von Stamm T268 erhalten werden. Neben Ubichinon Q8 (27) wurden zusätzlich Ubichinon KQ2 (30) und Ubichinon KQ5 (28) isoliert. Die Verbindungen 28 und 30 stellen Vertreter eines neuen Typs von Prenylchinonen dar, deren Biosynthese in der vorliegenden Arbeit diskutiert wird.<smiles>COC1=C(OC)C(=O)C(CC=C(C)CCC=C(C)C)=C(C)C1=O</smiles>

$27 \mathrm{n}=7$<smiles>COC1=C(OC)C(=O)C(CC=C(C)CCCC(C)=O)=C(C)C1=O</smiles>

$28 n=4$

$30 \mathrm{n}=1$

Der Rohextrakt des aus dem arktischen Meereis isolierten psychrotoleranten Stammes Salegentibacter holothuriorum T436 war intensiv gelb gefärbt. Es konnten daraus neben mehreren einfachen niedermolekularen Substanzen 24 aromatische Nitroverbindungen aus unterschiedlichen Substanzklassen isoliert werden. Zehn dieser Verbindungen waren bisher nicht literaturbekannt, drei Substanzen lediglich als Syntheseprodukte beschrieben. Größtenteils handelte es sich um substituierte Mononitro- sowie Dinitrophenole. Interessanterweise wurden auch Nährmedienbestandteile in nitrierter Form gefunden, und zwar 6-Nitro-cyclo(tyrosylprolyl) (55), 3'-Nitro-daidzein (56) und 3',5'-Dinitro-genistein (57).<smiles>O=C1NC(Cc2ccc(O)c([N+](=O)[O-])c2)C(=O)N2CCCC12</smiles><smiles>O=c1c(-c2ccc(O)c([N+](=O)[O-])c2)coc2cc(O)ccc12</smiles><smiles>O=c1c(-c2cc([N+](=O)[O-])c(O)c([N+](=O)[O-])c2)coc2cc(O)cc(O)c12</smiles> 
Stamm T436 erwies sich zudem als Produzent mehrerer nitrierter Indol-Derivate. So konnten die Verbindungen 2-Nitro- $N_{\beta}$-acetyltryptamin (58), 6-Nitro- $N_{\beta}$-acetyltryptamin (59) und 7-Nitro- $N_{\beta}$-acetyltryptamin (60) isoliert werden, welche sich lediglich in der Position der Nitro-Substitution unterscheiden. Bei den Verbindungen Salegentipyrrol A (61) und B (64) handelt es sich um Vertreter einer Substanzklasse, die biosynthetisch wahrscheinlich aus $N_{\beta}$-Acetyltryptamin durch Ringschluss und anschließende elektrophile Addition eines Nitroniumions entsteht. Alle nitrierten Indol-Derivate von Stamm T436 waren bisher unbekannt. Weitere Untersuchungen am Stamm T436 sind notwendig, um den biosynthetischen Ursprung der Nitrogruppen, für den verschiedene Mechanismen in Frage kommen, zu klären.

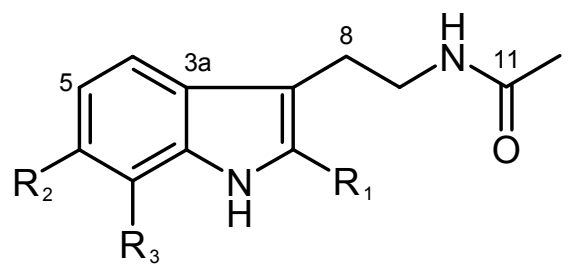

$58 \mathrm{R}_{1}=\mathrm{NO}_{2} ; \mathrm{R}_{2}=\mathrm{H} ; \mathrm{R}_{3}=\mathrm{H}$

$59 \mathrm{R}_{1}=\mathrm{H} ; \mathrm{R}_{2}=\mathrm{NO}_{2} ; \mathrm{R}_{3}=\mathrm{H}$

$\mathbf{6 0} \mathrm{R}_{1}=\mathrm{H} ; \mathrm{R}_{2}=\mathrm{H} ; \mathrm{R}_{3}=\mathrm{NO}_{2}$

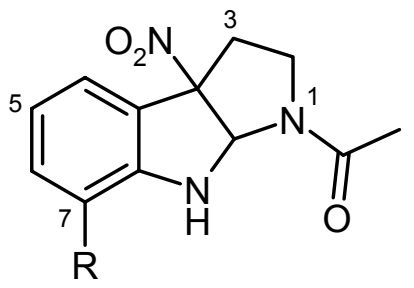

$61 \mathrm{R}=\mathrm{H}$

$62 \mathrm{R}=\mathrm{NO}_{2}$

Im Zuge der HPLC-ESI-MS/MS-Untersuchungen wurden im Rahmen dieser Arbeit zusätzlich die Fragmentierungswege der isolierten aromatischen Nitroverbindungen aufgeklärt. Im negativen Modus konnte in den meisten Fällen eine NO- sowie $\mathrm{HNO}_{2}$-Abspaltung detektiert werden, während im positiven Modus bevorzugt die Eliminierung von $\mathrm{NO}_{2}$ auftrat.

Der aus arktischen Pinguinexkrementen stammende, psychrophile Stamm T406 wurde bei einer Temperatur von $4{ }^{\circ} \mathrm{C}$ kultiviert. Aus dem erhaltenen cytotoxischen Rohextrakt konnte der Sekundärmetabolit SS-43405e (88) isoliert werden, der zur Substanzklasse der Pluramycine gehört. Zusätzlich wurde die bisher nicht bekannte Substanz 2-(Indol-3-yl-2'methyl)-indol-3-ylethanol (84) erhalten. Derartige, strukturell als 2,3-Bisindolylmethane zu klassifizierende Verbindungen, waren bisher lediglich aus der Hefe Malassezia furfur und dem thermophilen Archaebakterium Sulfolobus acidocaldarius bekannt. 
<smiles>CCC(C)c1cc(=O)c2c(C)cc3c(c2o1)C(=O)c1c(O)cccc1C3=O</smiles>

88<smiles>OCCc1c(Cc2c[nH]c3ccccc23)[nH]c2ccccc12</smiles>

84

Der Stamm T846 wurde aus einer Wasserprobe des Humboldt-Stroms vor der chilenischen Küste isoliert. Der nach der Kultivierung erhaltene Rohextrakt war cytotoxisch gegen mehrere Zelllinien aktiv und enthielt 1,3,5-Triazin (83) als ungewöhnlichen niedermolekularen Metaboliten. Die heterocyclische Verbindung $\mathbf{8 3}$ wurde bislang als Naturstoff nicht beschrieben. $\mathrm{Ob}$ die biologische Aktivität durch $\mathbf{8 3}$ oder andere Inhaltsstoffe bewirkt wurde, muss noch untersucht werden.<smiles>c1ncncn1</smiles>

83

Der Stamm Streptomyces flavogriseus ACT7651 wurde aus litoralem Sediment, das in der Nähe von Punta Arenas (Feuerland) gesammelt wurde, isoliert. Der durch Adsorption an XAD-16 erhaltene Rohextrakt dieses marinen Streptomyceten enthielt die bekannten Verbindungen Enterocin (95), 5-Desoxyenterocin (96) und Zimtsäureamid (99). Bei den beiden letztgenannten Verbindungen handelt es sich um Intermediate der EnterocinBiosynthese. Die nicht von XAD-16 adsorbierte wässrige Fraktion wurde mit Essigester getrennt extrahiert und lieferte das Makrolid Venturicidin A (100).

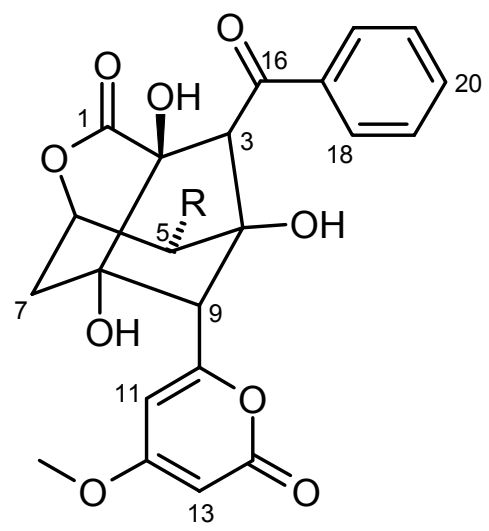

$95 \mathrm{R}=\mathrm{OH}$

$96 \mathrm{R}=\mathrm{H}$ 


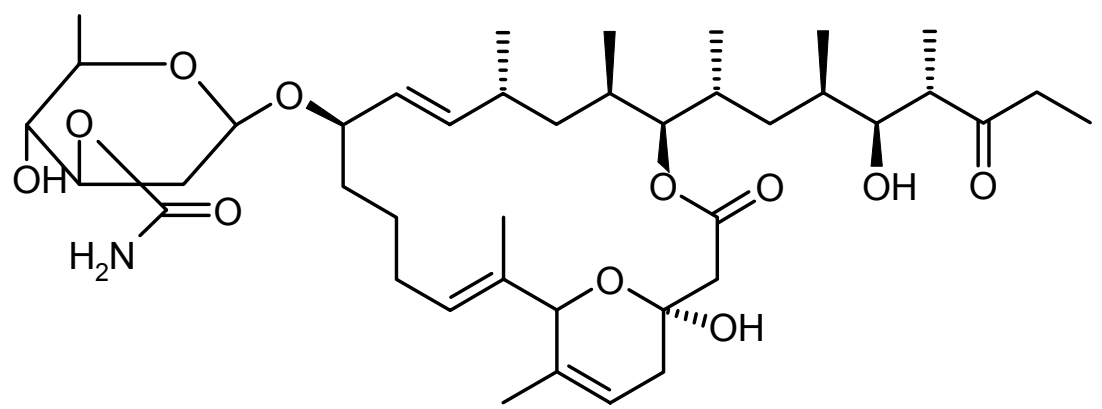

100

Die Sekundärmetaboliten des Stammes ACT7651 unterschieden sich im Hinblick auf Strukturgröße und Verbindungsklasse deutlich von den übrigen aus polaren Bakterien isolierten Verbindungen.

\section{Streptomyceten aus anderen Quellen}

Der nach sechstägiger Kultivierung erhaltene phycotoxische Rohextrakt des terrestrischen Actinomyceten Streptomyces sp. GW24/1811 enthielt das Anthracyclin Komodochinon B (104) und das pentacyclische Triterpenoid Hopen-B (101). Hopane sind Triterpene, die bei Bakterien ähnlich wie Cholesterol und Ergosterol bei Tieren und Pflanzen deren Zellmembran stabilisieren. Dieser an sich weit verbreitete Verbindungstyp wurde mit 101 in unserer Arbeitsgruppe hier erstmals isoliert.<smiles>C[C@]1(O)Cc2c(c(O)c3[14c](c2O)[C@H](O)C3=O)C(=O)c2cccc(O)c21</smiles>

104

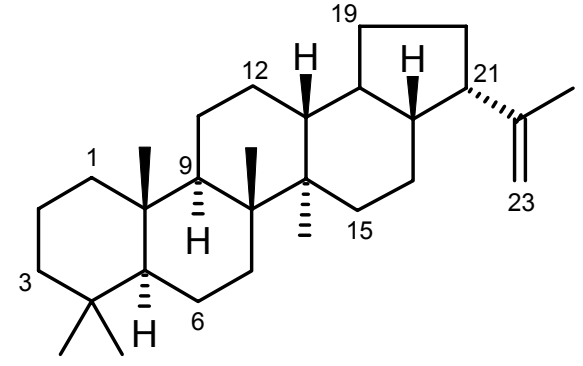

101

Die antibakterielle Wirkung des Rohextraktes aus dem terrestrischen Stamm Streptomyces sp. GW12/1492 konnte auf das Makrolid X-14952B (106) und das schwerlösliche Cervinomycin $A_{2}(\mathbf{1 0 7})$ zurückgeführt werden. Diese beiden Hauptmetaboliten konnten in ungewöhnlich hohen Ausbeuten von 100 bzw. 50 mg/l isoliert werden. 
<smiles>CCC(=O)C(CC)C(O)C(C)CC(C)C(OC(=O)CC1(O)CC=C(C)C(/C(C)=C/CCCC(/C=C/C(C)C(O)C(C)C(O)C(C)CC)OC2CC(OC(N)=O)C(O)CC(C)O2)O1)C(C)O</smiles>

106<smiles>COc1cc2oc3c(c(=O)c2cc1OC)C(=O)c1c(ccc2cc4c(c(O)c12)C(=O)N1CCOC1(C)C4)C3=O</smiles>

107

Aus dem antibakteriell wirksamen Rohextrakt des terrestrischen Stammes Streptomyces sp. GW51/456 konnten die zur Substanzklasse der Pluramycine gehörenden Verbindungen ßIndomycinon (111) und Saptomycin A (112) erhalten werden.<smiles>[R]C(C=CC)C([R])(C)c1cc(=O)c2c(C)cc3c(c2o1)C(=O)c1c(cc[14cH]c1O)C3=O</smiles>

$111 \mathrm{R}_{1}=\mathrm{OH} ; \mathrm{R}_{2}=\mathrm{H}$

$112 \mathrm{R}_{1}=\mathrm{H} ; \mathrm{R}_{2}=\mathrm{OH}$

Die beiden neuen Isochinolinchinone 4-Methylamino-7-methyl-isoquinolin-3,6-dion (113) und 4-Methylamino-7,8-dimethyl-isoquinolin-3,6-dion (114) konnten aus dem antibakteriell und phycotoxisch wirksamen Rohextrakt des marinen Nordsee-Stammes Streptomyces sp. Mei24 isoliert werden. Ähnliche Verbindungen sind aus tropischen Schwämmen bekannt, was auch dort einen mikrobiellen Ursprung vermuten lässt. 
<smiles>[R]c1c(C)ncc2c1C(=O)C=C(NC)C2=O</smiles>

Im Rahmen dieser Arbeit konnte gezeigt werden, dass marine Bakterien, insbesondere solche arktischen und antarktischen Ursprunges, zur Bildung biologisch aktiver und strukturell interessanter Metaboliten befähigt sind. Im Vergleich mit terrestrischen Streptomyceten, welche seit langem als Quelle biologisch wirksamer Sekundärmetabolite bekannt und nach wie vor Gegenstand intensiver Untersuchungen sind, neigen polare Bakterien in höherem Maße zur Produktion niedermolekularer Verbindungen. Der Anteil an neuen, bisher nicht beschriebenen Strukturen war bei den letztgenannten in dieser Arbeit jedoch deutlich größer. Die Bearbeitung der marinen Stämme erwies sich vielfach als schwierig, da nach der Kultivierung meist nur geringe Mengen an Rohextrakten erhalten werden konnten. Als Ursache hierfür können die nicht optimalen Anzuchtbedingungen im Labor angeführt werden, wobei hier insbesondere die Zusammensetzung der Nährmedien eine entscheidende Rolle spielen dürfte. Von den Ergebnisse der vorliegenden Arbeit lässt sich zusammenfassend der Schluss ziehen, dass die Erforschung des Metabolitenmusters polarer Mikroorganismen aus den bisher wenig untersuchten Kältegebieten der Pole sehr vielversprechend ist und in Zukunft verstärkt betrieben werden sollte. 


\section{$9 \quad$ Materialien und Methoden}

Drehwerte: CD-Polarimeter (Perkin-Elmer, Modell 241).

IR-Spektren: Perkin-Elmer 1600 Series FT-IR; Perkin-Elmer 297 Infrared Spectrophotometer; Beckman DU-640; Shimadzu FT-IR; (KBr-Presslinge und Film).

UV/VIS-Spektren: Perkin-Elmer Lambda 15 UV/VIS Spectrometer

${ }^{1}$ H-NMR-Spektren: Varian Unity 300 (300 MHz), Bruker AMX 300 (300 MHz), Varian INOVA $500(500 \mathrm{MHz})$, Varian Inova $600(600 \mathrm{MHz})$. Tetramethylsilan als der interne Standard.

${ }^{13}$ C-NMR-Spektren: Varian Mercury 300 (75.5 MHz), Varian Unity 300 (75.5 MHz), Bruker AMX 300 (75.5 MHz), Varian INOVA 500 (125.7 MHz), Varian Inova 600 (150.8 MHz).

Massenspektren: EI-MS: Finnigan MAT 95 (70 eV). Hochauflösung: Perfluorkerosin als Vergleichssubstanz. DCI-MS: Finnigan MAT $95(200 \mathrm{eV})$, Reaktandgas: $\mathrm{NH}_{3}$. ESI-MS: Finnigan LCQ. HR-ESI-MS: Bruker Apex-Q III, 7 Tesla.

HPLC-MS: Massenspektrometer: Finnigan LCQ; UV/VIS-Detektor: Finnigan Surveyor PDA Detector (Thermo Electron Corporation); HPLC-Pumpe: Rheos 4000 (Flux Instrument); Entgaser: ERC-3415 $\alpha$ (Flux Instruments); Autosampler: Jasco 851-AS Intelligent Sampler (Jasco); Steuersoftware HPLC: Janeiro (Flux Instruments); Datensystem: Xcalibur (Finnigan); Datenbanksoftware: MS-Manager (ACDLabs); Säulen: EC 125/2 Nucleosil 100-5 C18 (Macherey-Nagel), Synergi $4 \mu$ MAX-RP 80A, 150×2.00 mm $4 \mu$ micron (Phenomenex); Lösungsmittel: Methanol gradient grade für die Flüssigkeitschromatographie (Merck und Fluka). Programm: von $10 \%$ Methanol auf $100 \%$ Methanol in $20 \mathrm{~min}, 10 \mathrm{~min} 100 \%$ Methanol, von $100 \%$ Methanol auf $10 \%$ Methanol in 2 min.; Flussrate: $300 \mu \mathrm{l} / \mathrm{min}$.

\section{Materialien}

Dünnschichtchromatographie (DC): DC-Folien Polygram SIL G/UV 254 (Macherey-Nagel \& Co., Düren).

Präparative Dickschichtchromatographie (PDC): Kieselgel P/UV 254 (55 g/100 ml Wasser, Macherey-Nagel \& Co.) goß man auf waagrecht liegende Glasplatten $(20 \times 20 \mathrm{~cm})$, ließ an der Luft trocknen und aktivierte $4 \mathrm{~h}$ bei $130{ }^{\circ} \mathrm{C}$. 
Säulenchromatographie (SC): MN Kieselgel 60, 0.05-0.2 mm (Macherey-Nagel \& Co.); Kieselgel 60, 0.063-0.100 mm (Merck).

Ausschlußchromatographie: Sephadex LH-20 (Fluka).

\section{Sprühreagenzien}

Anisaldehyd/Schwefelsäure: $\mathrm{Zu} 100 \mathrm{ml}$ einer Stammlösung aus $85 \mathrm{ml}$ Methanol, $14 \mathrm{ml}$ Eisessig und $1 \mathrm{ml}$ Schwefelsäure fügte man $1 \mathrm{ml}$ Anisaldehyd hinzu.

Ehrlichs Reagenz: $1 \mathrm{~g}$ 4-Dimethylaminobenzaldehyd wird in einer Mischung aus $25 \mathrm{ml}$ Salzsäure (36 \%) und $75 \mathrm{ml}$ Methanol gelöst.

\section{Zinn(II)-chlorid-Salzsäure/4-Dimethylaminobenzaldehyd:}

Sprühlösung I: $3 \mathrm{ml}$ Zinn(II)-chlorid-Lösung (15\% in Wasser) werden mit $15 \mathrm{ml}$ Salzsäure (37 \%) gemischt und mit $180 \mathrm{ml}$ Wasser verdünnt. Die Lösung wurde frisch zubereitet.

Sprühlösung II: $1 \mathrm{~g}$ 4-Dimethylaminobenzaldehyd wird in einer Mischung aus $30 \mathrm{ml}$ Ethanol, $3 \mathrm{ml}$ Salzsäure (37\%) und $180 \mathrm{ml} \mathrm{1-Butanol} \mathrm{gelöst.}$

Vorgang: Mit I sprühen, an der Luft trocknen und mit II nachsprühen.

\section{Mikrobiologische Materialien}

Fermenter: 20-l-Fermenter (Meredos GmbH, Göttingen) bestehend aus Kulturgefäß, Magnetgekuppeltem Propellerrührer, Kühlschlange mit Thermostat, Steuereinheit mit pHRegler, Antischaum-Regler (Meredos GmbH, Göttingen).

Schüttler: Infors AG (CH 4103 Einbach) Typ ITE.

Autoklav: Albert Dargatz Autoklav, Volumen 119 l, Betriebstemperatur $121^{\circ} \mathrm{C}$, Betriebsdruck $1.2 \mathrm{~kg} / \mathrm{cm}^{2}$

Stammhaltung: Tiefkühllagerung in Dewargefäß Typ BT 37 Air liquid

Kapillaren zur Tiefkühllagerung: Durchmesser $1.75 \mathrm{~mm}$, Länge $80 \mathrm{~mm}$ (Hirschmann Laborgeräte, Eberstadt)

Erde für Erdkultur: Luvos Heilerde (LU-VOSJUST GmbH \& Co., Friedrichshof)

Ultraturrax: Janke \& Munkel KG 
Testplättchen: 9 mm Durchmesser, Schleicher \& Schüll No. 321261

Nährbodenbestandteile: Glucose, Hefeextrakt und Malzextrakt (Merck, Darmstadt), Bacto Agar (Difco)

Antischaum-Lösung: Niax PPG 2025 (Union Carbide Belgium N. V., Zwiijndrecht)

Petrischalen: Durchmesser 94 mm, Höhe 16 mm (Greiner Labortechnik, Nürtingen)

Celite: Celite France S. A., Rueil-Malmaison Cedex

\section{Rezepturen}

Alle Kultur-Medien wurden bei 1.2 bar und $121{ }^{\circ} \mathrm{C}$ autoklaviert. Die Verweildauer im Autoklav betrug für 1-1-Schüttelkulturen 33 min, für Fermenter-Medien in Form eines 2 1- Konzentrats 50 min und für den mit 16 I Wasser gefüllten Fermentationsbehälter 82 min.

\section{Künstliches Seewasser (A)}

Eisencitrat (vorher zermörsert)

$$
\begin{array}{r}
2 \mathrm{~g} \\
389 \mathrm{~g} \\
176 \mathrm{~g} \\
68.8 \mathrm{~g} \\
36 \mathrm{~g} \\
0.16 \mathrm{~g} \\
0.3 \mathrm{~g} \\
20 \mathrm{ml} \\
200 \mathrm{ml}
\end{array}
$$$$
\mathrm{NaCl}
$$$$
\mathrm{MgCl}_{2} \cdot 6 \mathrm{H}_{2} \mathrm{O}
$$$$
\mathrm{Na}_{2} \mathrm{SO}_{4} \text { (vorher in } 21 \text { Wasser }
$$$$
\text { aufgelöst) }
$$$$
\mathrm{CaCl}_{2}
$$

$\mathrm{Na}_{2} \mathrm{HPO}_{4}$

$\mathrm{SiO}_{2}$

Spurenelement-Lösung

Stamm-Lösung

Die Lösungen wurden mit Leitungswasser auf 201 aufgefüllt.

\section{Spurenelement-Lösung nach Hoagland (1993)}

$\begin{array}{ll}\mathrm{H}_{3} \mathrm{BO}_{3} & 0.611 \mathrm{~g} \\ \mathrm{MnCl}_{2} & 0.389 \mathrm{~g} \\ \mathrm{CuSO}_{4} & 0.056 \mathrm{~g} \\ \mathrm{ZnSO}_{4} \cdot 7 \mathrm{H}_{2} \mathrm{O} & 0.056 \mathrm{~g} \\ \mathrm{Al}_{2}\left(\mathrm{SO}_{4}\right)_{3} \cdot 18 \mathrm{H}_{2} \mathrm{O} & 0.056 \mathrm{~g} \\ \mathrm{NiSO}_{4} \cdot 6 \mathrm{H}_{2} \mathrm{O} & 0.056 \mathrm{~g}\end{array}$




$\begin{array}{ll}\mathrm{Co}\left(\mathrm{NO}_{3}\right)_{3} \cdot 6 \mathrm{H}_{2} \mathrm{O} & 0.056 \mathrm{~g} \\ \mathrm{TiO}_{2} & 0.056 \mathrm{~g} \\ \left(\mathrm{NH}_{4}\right)_{6} \mathrm{Mo}_{7} \mathrm{O}_{24} \cdot 4 \mathrm{H}_{2} \mathrm{O} & 0.056 \mathrm{~g} \\ \mathrm{LiCl} & 0.028 \mathrm{~g} \\ \mathrm{SnCl}_{2} & 0.028 \mathrm{~g} \\ \mathrm{KI} & 0.028 \mathrm{~g}\end{array}$

Die Spurenelementsalze wurden in 11 Leitungswasser gelöst.

\section{Stamm-Lösung}

$\begin{array}{lr}\mathrm{KCl} & 110 \mathrm{~g} \\ \mathrm{NaHCO}_{3} & 32 \mathrm{~g} \\ \mathrm{KBr} & 16 \mathrm{~g} \\ \mathrm{SrCl}_{2} \cdot 6 \mathrm{H}_{2} \mathrm{O} \text { (getrennt aufgelöst) } & 6.8 \mathrm{~g} \\ \mathrm{H}_{3} \mathrm{BO}_{3} & 4.4 \mathrm{~g} \\ \mathrm{NaF} & 0.48 \mathrm{~g} \\ \mathrm{NH}_{4} \mathrm{NO}_{3} & 0.32 \mathrm{~g}\end{array}$

Die Salze wurden mit Leitungswasser auf 21 aufgefüllt.

\section{Künstliches Seewasser (B)}

$\mathrm{NaCl}$

$24 \mathrm{~g}$

$\mathrm{MgCl}_{2}$ $5 \mathrm{~g}$

$\mathrm{MgSO}_{4}$ $5 \mathrm{~g}$

$\mathrm{CaCl}_{2}$ $0.5 \mathrm{~g}$

$\mathrm{KCl}$

$0.5 \mathrm{~g}$

$\mathrm{KBr}$

$0.1 \mathrm{~g}$

$\mathrm{FePO}_{4} \cdot \mathrm{H}_{2} \mathrm{O}$

$10 \mathrm{mg}$

demineralisiertes Wasser $1000 \mathrm{ml}$

\section{M1-Medium}

Pepton $5 \mathrm{~g}$

Hefe-Extrakt

$1 \mathrm{~g}$

$\mathrm{KBr}$

$1 \mathrm{~g}$

Seewasser $750 \mathrm{ml}$

demineralisiertes Wasser $250 \mathrm{ml}$

Die Lösung wurde mit $2 \mathrm{~N} \mathrm{NaOH}$ auf pH 8.0 eingestellt. 


\section{$M_{2}$ ohne Seewasser}

Malz-Extrakt

$10 \mathrm{~g}$

Glucose

$4 \mathrm{~g}$

Hefe-Extrakt

$4 \mathrm{~g}$

Leitungswasser

$1000 \mathrm{ml}$

Die Lösung wurde mit $2 \mathrm{~N} \mathrm{NaOH}$ auf $\mathrm{pH} 7.8$ eingestellt. Bei festem Medium wurden zusätzlich $18 \mathrm{~g}$ Bacto Agar zugegeben.

\section{$\mathbf{M}_{2}$ mit Seewasser}

Malz-Extrakt

$$
\begin{array}{r}
10 \mathrm{~g} \\
4 \mathrm{~g} \\
4 \mathrm{~g} \\
500 \mathrm{ml} \\
500 \mathrm{ml}
\end{array}
$$

Glucose

Hefe-Extrakt

künstliches Seewasser

Leitungswasser

Die Lösung wurde mit $2 \mathrm{~N} \mathrm{NaOH}$ auf $\mathrm{pH} 7.8$ eingestellt. Bei festem Medium wurden zusätzlich $18 \mathrm{~g}$ Bacto Agar zugegeben.

\section{LB-Medium}

Trypton

$$
\begin{array}{r}
10 \mathrm{~g} \\
5 \mathrm{~g} \\
10 \mathrm{~g} \\
500 \mathrm{ml} \\
500 \mathrm{ml}
\end{array}
$$

Hefe-Extrakt

$\mathrm{NaCl}$

künstliches Seewasser (B)

demineralisiertes Wasser

Die Lösung wurde mit $2 \mathrm{~N} \mathrm{NaOH}$ auf $\mathrm{pH} 7.6 \pm 0.2$ eingestellt.

\section{B-Medium}

Maisstärke (Merck)

$$
5 \mathrm{~g}
$$

N-Z-Amine (Sigma)

$2,5 \mathrm{~g}$

Rindfleischextrakt (Difco)

$3,8 \mathrm{~g}$

Sojamehl (Sojamin 50T)

Hefeextrakt (Difco)

$2,5 \mathrm{~g}$

$\mathrm{KNO}_{3}$

Seetangextrakt (Manufactum) $2,5 \mathrm{ml}$

Seewassermischung (Tropic Marin) 
demineralisiertes Wasser

$1000 \mathrm{ml}$

Die Lösung wurde auf pH 8.0 eingestellt.

\section{Fleischextrakt-Medium}

Pepton

$2 \mathrm{~g}$

Glucose

$10 \mathrm{~g}$

Hefe-Extrakt

$1 \mathrm{~g}$

Fleischextrakt

$1 \mathrm{~g}$

Leitungswasser

$1000 \mathrm{ml}$

Die Lösung wurde mit $2 \mathrm{~N} \mathrm{NaOH}$ auf pH 7.8 eingestellt.

\section{Medium für Nordsee-Streptomyceten ("Mei-Stämme")}

Malz-Extrakt

Glucose. $\mathrm{H}_{2} \mathrm{O}$

Hefe-Extrakt

$\mathrm{CaCO}_{3}$

$13.2 \%$ Seewasser

\author{
$10 \mathrm{~g}$ \\ $4 \mathrm{~g}$ \\ $4 \mathrm{~g}$ \\ $0.5 \mathrm{~g}$ \\ $1000 \mathrm{ml}$
}

Die Lösung wurde mit $2 \mathrm{~N} \mathrm{NaOH}$ auf pH 7.3 eingestellt.

\section{LB-Agar}

Trypton

$5 \mathrm{~g}$

Hefeextrakt $5 \mathrm{~g}$

$\mathrm{NaCl}$ $10 \mathrm{~g}$

Agar $20 \mathrm{~g}$

demineralisiertes Wasser $1000 \mathrm{ml}$

Die Lösung wurde auf pH 7.2 eingestellt.

\section{LBm-Agar}

Trypton $5 \mathrm{~g}$

Hefeextrakt $5 \mathrm{~g}$

$\mathrm{NaCl}$ $10 \mathrm{~g}$

Agar $20 \mathrm{~g}$

demineralisiertes Wasser $500 \mathrm{ml}$

Seewasser $500 \mathrm{ml}$

Die Lösung wurde auf pH 7.2 eingestellt. 


\section{Nutrient-broth-Agar}

Nutrient broth (Difco)

$8 \mathrm{~g}$

Agar

$20 \mathrm{~g}$

demineralisiertes Wasser

$1000 \mathrm{ml}$

Die Lösung wurde auf $\mathrm{pH} 7.6$ eingestellt.

\section{M-Test-Agar}

(für die Testkeime Escherichia coli, Bacillus subtilis (ATCC 6051), Staphylococcus aureus, Mucor miehei (Tü 284))

Malz-Extrakt

$10 \mathrm{~g}$

Glucose

$4 \mathrm{~g}$

Hefe-Extrakt

$4 \mathrm{~g}$

Bacto Agar

$20 \mathrm{~g}$

demineralisiertes Wasser

$1000 \mathrm{ml}$

Die Lösung wurde mit $2 \mathrm{~N} \mathrm{NaOH}$ auf pH 7.3 eingestellt.

\section{Sabouraud-Agar}

(für den Testkeim Candida albicans)

$\begin{array}{lr}\text { Glucose } & 40 \mathrm{~g} \\ \text { Bacto Pepton } & 10 \mathrm{~g} \\ \text { Bacto Agar } & 20 \mathrm{~g} \\ \text { demineralisiertes Wasser } & 1000 \mathrm{ml}\end{array}$

Die Lösung wurde mit $2 \mathrm{~N} \mathrm{NaOH}$ auf pH 7.3 eingestellt.

\section{Bolds Basal Medium (BBM):}

Medium für die Algenstämme Chlorella sorokiniana, Chlorella vulgaris und Scenedesmus subspicatus

$\begin{array}{lr}\mathrm{NaNO}_{3} & 250 \mathrm{mg} \\ \mathrm{KH}_{2} \mathrm{PO}_{4} & 175 \mathrm{mg} \\ \mathrm{K}_{2} \mathrm{HPO}_{4} & 75 \mathrm{mg} \\ \mathrm{MgSO}_{4} \cdot 7 \mathrm{H}_{2} \mathrm{O} & 75 \mathrm{mg} \\ \mathrm{NaCl} & 25 \mathrm{mg} \\ \mathrm{CaCl}_{2} \cdot 2 \mathrm{H}_{2} \mathrm{O} & 25 \mathrm{mg} \\ \mathrm{Fe}-\mathrm{EDTA} & 1.0 \mathrm{ml}\end{array}$


Spurenelementlösung II

$1.0 \mathrm{ml}$

Die Salze wurden jeweils in $10 \mathrm{ml} \mathrm{H}_{2} \mathrm{O}$ gelöst. Dieses Lösungen vereinigte man mit der FeEDTA-Lösung und der Spurenelementlösung II und füllte auf $1000 \mathrm{ml}$ mit demineralisiertem Wasser auf. Bei festem Medium 15 g Agar-Zusatz.

\section{Fe-EDTA-Lösung}

Man löste $0.7 \mathrm{~g} \mathrm{FeSO}_{4} \cdot 7 \mathrm{H}_{2} \mathrm{O}$ und $0.93 \mathrm{~g}$ EDTA (Titriplex III) in $80 \mathrm{ml} \mathrm{H} \mathrm{H}_{2} \mathrm{O}$ unter Erwärmen.

Diese Lösung wurde mit demineralisiertem Wasser auf $100 \mathrm{ml}$ aufgefüllt.

\section{Spurenelementlösung II}

\section{Lösung A:}
$\mathrm{MnSO}_{4} \cdot \mathrm{H}_{2} \mathrm{O}$
$16.9 \mathrm{mg}$
$\mathrm{Na}_{2} \mathrm{MoO}_{4} \cdot 2 \mathrm{H}_{2} \mathrm{O}$
$13.0 \mathrm{mg}$
$\mathrm{Co}\left(\mathrm{NO}_{3}\right)_{2} \cdot 6 \mathrm{H}_{2} \mathrm{O}$
$10.0 \mathrm{mg}$

Die Salze wurden jeweils in $10 \mathrm{ml}$ demineralisiertem Wasser gelöst. Diese Lösungen wurden vereinigt.

Lösung B:
$\mathrm{CuSO}_{4} \cdot 5 \mathrm{H}_{2} \mathrm{O}$
$5.0 \mathrm{mg}$
$\mathrm{H}_{3} \mathrm{BO}_{3}$
$10.0 \mathrm{mg}$
$\mathrm{ZnSO}_{4} \cdot 7 \mathrm{H}_{2} \mathrm{O}$
$10.0 \mathrm{mg}$

Die Salze wurden in $10 \mathrm{ml}$ demineralisiertem Wasser gelöst.

Lösung A und B wurden vereinigt und mit demineralisiertem Wasser auf $100 \mathrm{ml}$ aufgefüllt. 


\section{$10 \quad$ Experimenteller Teil}

\subsection{Pseudoalteromonas sp. T268}

Der aus dem Darminhalt der antarktischen Krillart Euphausia superba isolierte Stamm T268 bildete beige-farbene, glänzende Kolonien.

\subsubsection{Primärscreening}

Im Dünnschichtchromatogramm fielen lediglich zwei Zonen auf, die weder UV-löschend noch fluoreszierend waren. Nach Besprühen mit Anisaldehyd und Erwärmung zeigte eine der Zonen eine blaue Anfärbung. Die andere Zone färbte sich weder mit Anisaldehyd noch mit Ehrlichs Reagenz an. Im biologischen Screening (300 $\mu \mathrm{g} /$ Plättchen), das am Institut für Biotechnologie und Wirkstoffforschung (Kaiserslautern) durchgeführt wurde, zeigte der Rohextrakt des Stammes keine auffälligen Aktivitäten.

Tabelle 8 Aktivität des Rohextraktes von Pseudoalteromonas sp. T268 im biologischen Screening.

\begin{tabular}{|l|c|}
\hline \multicolumn{1}{|c|}{ Testkeim } & Hemmhof $\varnothing[\mathrm{mm}]$ \\
\hline \hline Bacillus brevis (ATCC 9999) & 10 \\
\hline Bacillus subtilis (ATCC 6633) & 10 \\
\hline Nematospora coryli (ATCC 10647) & 10 \\
\hline Micrococcus luteus (ATCC 381) & 15 \\
\hline Mucor miehei (Tü 284) & 0 \\
\hline Paecilomyces variotii (ETH 114646) & 0 \\
\hline
\end{tabular}

\subsubsection{Kultivierung und Aufarbeitung}

Die Kultivierung von Stamm T268 in einem 20 l-Fermenter und wurde ebenfalls am Institut für Biotechnologie und Wirkstoff-Forschung (Kaiserslautern) durchgeführt. Die Fermentation erfolgte in $\mathrm{M}_{1}$-Medium, wobei zum Animpfen $250 \mathrm{ml}$ einer $24 \mathrm{~h}$ alten Vorkultur dienten. Für die Kultivierung wurden ein Belüftungsdruck von 2.5 l/min, eine Rührgeschwindigkeit von $121 \mathrm{Upm}$ sowie eine Temperatur von $20 \pm 0.5^{\circ} \mathrm{C}$ gewählt. 
Die Kulturbrühe wurde nach Zusatz von Celite mittels einer Filterpresse in Mycel und Kulturfiltrat getrennt. Letzteres wurde dreimal mit Essigester extrahiert. Das Mycel wurde mit Essigester versetzt und nach Ultraschall-Behandlung filtriert. Die vereinigten organischen Phasen wurden anschließend vom Lösungsmittel befreit, wobei sich $4.5 \mathrm{~g}$ Rohextrakt ergaben.

\subsubsection{Isolierung und Charakterisierung der Metaboliten}

Der Rohextrakt wurde mittels Säulenchromatographie an Kieselgel unter Verwendung eines Stufengradienten aus Dichlormethan/Methanol $\left(\mathrm{CH}_{2} \mathrm{Cl}_{2}, \mathrm{CH}_{2} \mathrm{Cl}_{2} / \mathrm{MeOH}\right.$ 99:1, $\mathrm{CH}_{2} \mathrm{Cl}_{2} / \mathrm{MeOH}$ 98:2, $\mathrm{CH}_{2} \mathrm{Cl}_{2} / \mathrm{MeOH}$ 97:3, $\mathrm{CH}_{2} \mathrm{Cl}_{2} / \mathrm{MeOH}$ 96:4, $\mathrm{CH}_{2} \mathrm{Cl}_{2} / \mathrm{MeOH}$ 95:5, $\mathrm{CH}_{2} \mathrm{Cl}_{2} / \mathrm{MeOH}$ 9:1, $\mathrm{CH}_{2} \mathrm{Cl}_{2} / \mathrm{MeOH}$ 4:1) getrennt. Die so erhaltenen Fraktionen wurden durch Chromatographie an Sephadex ${ }^{\circledR}$ LH-20 $\left(\mathrm{CH}_{2} \mathrm{Cl}_{2} / \mathrm{MeOH}\right.$ 3:2 und $\left.\mathrm{MeOH}\right)$ gereinigt. Erneute Flashchromatographie an Kieselgel $\left(\mathrm{CH}_{2} \mathrm{Cl}_{2} / \mathrm{MeOH}\right)$ lieferte $6.9 \mathrm{mg}$ Isatin (19), $3.1 \mathrm{mg}$ 3-Hydroxy-3-acetonyloxindol (20), 2.0 mg 3-Hydroxy-3-(1-propanonyl)oxindol (21), $2.3 \mathrm{mg}$ Ubichinon Q8 (27), $1.2 \mathrm{mg}$ Ubichinon KQ5 (29), 0.8 mg Ubichinon KQ2 (30), 1.3 mg Norharman (31), 0.3 mg Harman (31), 120 mg Glutarsäure (32), $31.2 \mathrm{mg}$ Monomethylglutarsäure (32), $0.5 \mathrm{mg}$ Indolcarbonsäure (33), 1.7 mg 3-Hydroxyacetylindol (34), $1.9 \mathrm{mg}$ Uracil (35), $40 \mathrm{mg}$ p-Hydroxybenzoesäure (36), $3.3 \mathrm{mg}$ Tyrosol (37), $0.8 \mathrm{mg}$ Vanillinsäure (38) und $1.6 \mathrm{mg}$ 4-Hydroxy-3methoxy-phenyl-propionsäure (39).

Isatin (19): $\mathrm{C}_{8} \mathrm{H}_{5} \mathrm{NO}_{2}$ (147), oranger Feststoff, UV-löschend. - $\boldsymbol{R}_{\boldsymbol{f}}$ $=0.35\left(\mathrm{CH}_{2} \mathrm{Cl}_{2} / \mathrm{MeOH}\right.$ 95:5). - Anfärbeverhalten: Anisaldehyd (gelb), Ehrlich (orange). $-{ }^{1} \mathbf{H}-\mathbf{N M R}\left(\mathrm{CDCl}_{3}, 300 \mathrm{MHz}\right): \delta=7.81$ (br, 1H, 1-NH), 7.63 (d, $\left.{ }^{3} J=7.9 \mathrm{~Hz}, 1 \mathrm{H}, 4-\mathrm{H}\right), 7.57$ (t, ${ }^{3} J=7.8 \mathrm{~Hz}$,

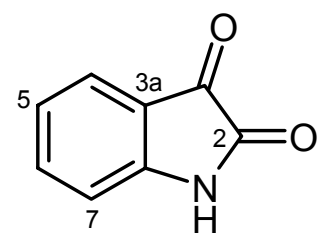
$1 \mathrm{H}, 6-\mathrm{H}), 7.14\left(\mathrm{t},{ }^{3} J=8.0 \mathrm{~Hz}, 1 \mathrm{H}, 5-\mathrm{H}\right), 6.90\left(\mathrm{~d},{ }^{3} \mathrm{~J}=8.0 \mathrm{HZ}, 1 \mathrm{H}\right.$, 7-H). - EI-MS (70 eV): $m / z(\%)=147\left([\mathrm{M}]^{\bullet+}, 43\right), 119$ (100), 92 (74), 64 (39).

3-Hydroxy-3-acetonyloxindol (20): $\mathrm{C}_{11} \mathrm{H}_{11} \mathrm{NO}_{3} \quad$ (205), weißer Feststoff, UV-löschend $(254 \mathrm{~nm}) . \quad-\boldsymbol{R}_{\boldsymbol{f}}=0.23$ $\left(\mathrm{CH}_{2} \mathrm{Cl}_{2} / \mathrm{MeOH}\right.$ 95:5). - Anfärbeverhalten: Anisaldehyd (violett), Ehrlich (grün). $-\boldsymbol{R}_{t}=7.0 \mathrm{~min}$ (LC-MS). $-{ }^{\mathbf{1}} \mathbf{H}-$<smiles>CC(=O)CC1(O)C(=O)Nc2ccccc21</smiles>
NMR $\left(\mathrm{CDCl}_{3}, 300 \mathrm{MHz}\right): \delta=7.78$ (s br, 1H, 3-OH), 7.37 (d, $\left.{ }^{3} J=8.2 \mathrm{~Hz}, 1 \mathrm{H}, 4-\mathrm{H}\right), 7.28\left(\mathrm{t},{ }^{3} J=7.9 \mathrm{~Hz}, 1 \mathrm{H}, 6-\mathrm{H}\right), 7.06\left(\mathrm{t},{ }^{3} J=8.1 \mathrm{~Hz}, 1 \mathrm{H}, 5-\mathrm{H}\right), 6.89$ 
$\left(\mathrm{d},{ }^{3} J=8.0 \mathrm{~Hz}, 1 \mathrm{H}, 7-\mathrm{H}\right), 3.21,2.99\left(\mathrm{AB},{ }^{2} J=15.2 \mathrm{~Hz}, 2 \mathrm{H}, 8-\mathrm{H}\right), 2.21(\mathrm{~s}, 3 \mathrm{H}, 10-\mathrm{H}) .-{ }^{13} \mathrm{C}-$ NMR ([D $]$ DMSO, 75.5 MHz): $\delta=205.0\left(\mathrm{C}_{\mathrm{q}}, \mathrm{C}-9\right), 178.0\left(\mathrm{C}_{\mathrm{q}}, \mathrm{C}-2\right), 142.4\left(\mathrm{C}_{\mathrm{q}}, \mathrm{C}-7 \mathrm{a}\right), 131.4$ $\left(\mathrm{C}_{\mathrm{q}}, \mathrm{C}-3 \mathrm{a}\right), 128.9$ (CH, C-6), $123.6(\mathrm{CH}, \mathrm{C}-4), 121.1(\mathrm{CH}, \mathrm{C}-5), 109.3(\mathrm{CH}, \mathrm{C}-7), 72.6\left(\mathrm{C}_{\mathrm{q}}, \mathrm{C}-\right.$ 3), $50.2\left(\mathrm{CH}_{2}, \mathrm{C}-8\right), 30.5\left(\mathrm{CH}_{3}, \mathrm{C}-10\right)$. - EI-MS $(70 \mathrm{eV}): m / z(\%)=205\left(\left([\mathrm{M}]^{\circ+}, 19\right), 162\right.$ (26), 148 (29), 138 (24), 120 (27), 107 (100), 100 (56), 43 (21). ). - UV (MeOH): $\lambda_{\max }(\lg \varepsilon)=$ 216 (4.01), 255 (3.69), 291 (3.07) nm. - IR (KBr): $\widetilde{v}\left(\mathrm{~cm}^{-1}\right)=3371,3319,1720,1622,1486$, $1472,1403,1389,1363,1335,1228,1183,1115,1061,780,761,670,642,622,593,555$. ). $-[\alpha]_{D}^{20}=+1.94(\mathrm{c}=1 \mathrm{mg} / \mathrm{ml}, \mathrm{MeOH})$.

3-Hydroxy-3-(1-propanonyl)oxindol (21): $\mathrm{C}_{12} \mathrm{H}_{13} \mathrm{NO}_{3}$ (219), weißer Feststoff, UV-löschend (254 nm). $-\boldsymbol{R}_{\boldsymbol{f}}=$ $0.25\left(\mathrm{CH}_{2} \mathrm{Cl}_{2} / \mathrm{MeOH}\right.$ 95:5). - Anfärbeverhalten: Anisaldehyd (hellgrün), Ehrlich (hellrot). $-\boldsymbol{R}_{\boldsymbol{t}}=9.4 \mathrm{~min}$ (LC-

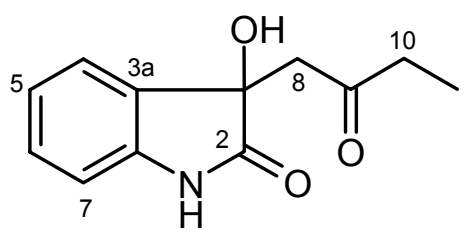
MS). - ${ }^{1} \mathbf{H}-\mathbf{N M R}\left(\mathrm{CDCl}_{3}, 300 \mathrm{MHz}\right): \delta=8.25(\mathrm{br}, 1 \mathrm{H}$, 3-OH), 7.30 (d, $\left.{ }^{3} J=7.5 \mathrm{~Hz}, 1 \mathrm{H}, 4-\mathrm{H}\right), 7.24\left(\mathrm{t},{ }^{3} J=7.9 \mathrm{~Hz}, 1 \mathrm{H}, 6-\mathrm{H}\right), 7.02\left(\mathrm{t},{ }^{3} J=7.3 \mathrm{~Hz}, 1 \mathrm{H}\right.$, $5-\mathrm{H}), 6.86\left(\mathrm{~d},{ }^{3} J=8.0 \mathrm{~Hz}, 1 \mathrm{H}, 7-\mathrm{H}\right), 3.13,2.94\left(\mathrm{AB},{ }^{2} J=15.2 \mathrm{~Hz}, 2 \mathrm{H}, 8-\mathrm{H}\right), 2.44\left(\mathrm{q},{ }^{3} J=7.3\right.$ $\mathrm{Hz}, 2 \mathrm{H}, 10-\mathrm{H}), 1.00\left(\mathrm{t},{ }^{3} J=7.3 \mathrm{~Hz}, 3 \mathrm{H}, 11-\mathrm{H}\right) .-{ }^{13} \mathbf{C}-\mathbf{N M R}\left(\mathrm{CD}_{3} \mathrm{OD}, 75 \mathrm{MHz}\right): \delta=209.8$ $\left(\mathrm{C}_{\mathrm{q}}, \mathrm{C}-9\right), 181.2\left(\mathrm{C}_{\mathrm{q}}, \mathrm{C}-2\right), 143.6\left(\mathrm{C}_{\mathrm{q}}, \mathrm{C}-7 \mathrm{a}\right), 132.3\left(\mathrm{C}_{\mathrm{q}}, \mathrm{C}-3 \mathrm{a}\right), 130.7(\mathrm{CH}, \mathrm{C}-6), 124.8(\mathrm{CH}$, C-4), $123.4(\mathrm{CH}, \mathrm{C}-5), 111.2(\mathrm{CH}, \mathrm{C}-7), 74.8\left(\mathrm{C}_{\mathrm{q}}, \mathrm{C}-3\right), 50.0\left(\mathrm{CH}_{2}, \mathrm{C}-8\right), 37.3\left(\mathrm{CH}_{2}, \mathrm{C}-10\right)$, $7.7\left(\mathrm{CH}_{3}, \mathrm{C}-11\right)$.- EI-MS $(70 \mathrm{eV}): m / z(\%)=219\left(\left([\mathrm{M}]^{++}, 61\right), 174(21), 162(94), 140(100)\right.$, 126 (18), 120 (62), 92 (16), $57(22) .-[\alpha]_{D}^{20}=+10.26(\mathrm{c}=1 \mathrm{mg} / \mathrm{ml}, \mathrm{MeOH})$.

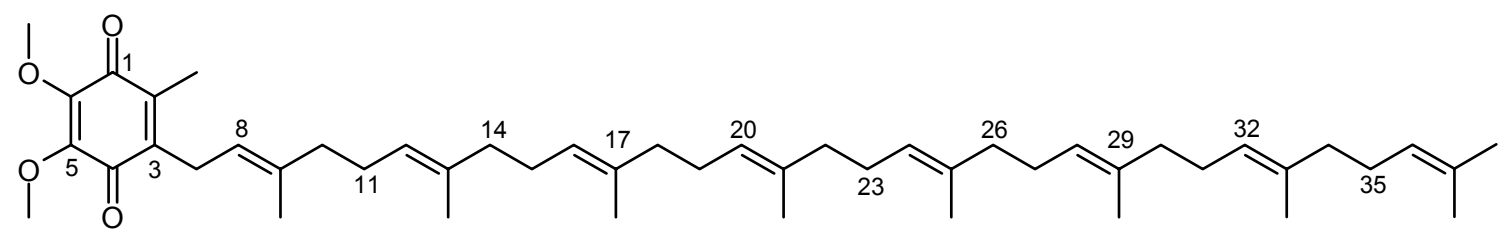

Ubichinon Q8 (27): $\mathrm{C}_{49} \mathrm{H}_{74} \mathrm{O}_{4}$ (726), gelbes Öl, UV-löschend. - $\boldsymbol{R}_{\boldsymbol{f}}=0.93\left(\mathrm{CH}_{2} \mathrm{Cl}_{2} / \mathrm{MeOH}\right.$ 95:5). - Anfärbeverhalten: Anisaldehyd (violet), Ehrlich (beige). - ${ }^{\mathbf{1}} \mathbf{H}-\mathbf{N M R}\left(\mathrm{CDCl}_{3}, 300\right.$ MHz): $\delta=5.09-5.06,5.04$ (m, t, 7H, 12-, 16-, 20-, 24-, 28-, 32, 36-H), 4.91 (t, 1H, 8-H), 3.97 $\left(\mathrm{s}, 3 \mathrm{H}, 5-\mathrm{OCH}_{3}\right), 3.96\left(\mathrm{~s}, 3 \mathrm{H}, 6-\mathrm{OCH}_{3}\right), 3.16(\mathrm{~d}, 2 \mathrm{H}, 7-\mathrm{H}), 2.05,1.94(2 \mathrm{~m}, 2 \times 14 \mathrm{H}, 10-, 11-$, 14-, 15-, 18-, 19-, 22-, 23-, 26-, 27-, 30-, 31-, 34-, 35-H), 1.99 (s, 3H, 2-H), 1.72 (s, 3H, 9$\left.\mathrm{CH}_{3}\right), 1.66\left(\mathrm{~s}, 3 \mathrm{H}, 13-\mathrm{CH}_{3}\right), 1.56\left(\mathrm{~s}, 15 \mathrm{H}, 17-, 21-, 25-, 29-, 33-\mathrm{CH}_{3}\right), 1.55\left(\mathrm{~s}, 6 \mathrm{H}, 37-\mathrm{CH}_{3}\right.$, 38-H). - (+)-ESI-MS: $m / z(\%)=1475[2 \mathrm{M}+\mathrm{Na}]^{+}(100), 749[\mathrm{M}+\mathrm{Na}]^{+}(18)$. 


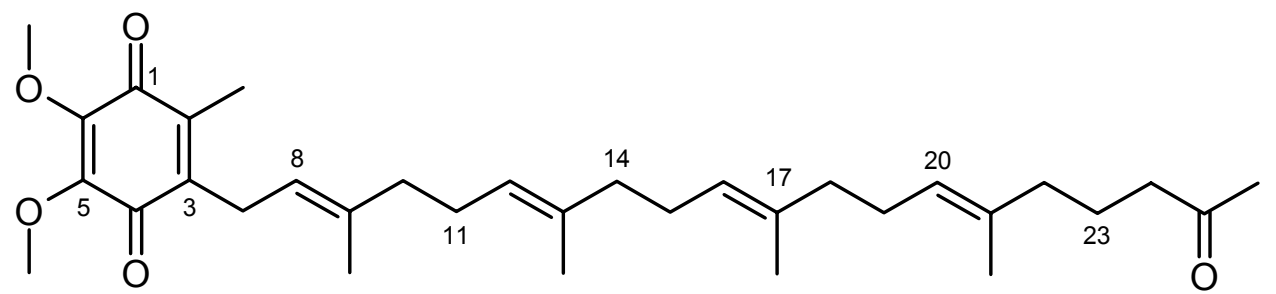

Ubichinon KQ5 (28): $\mathrm{C}_{33} \mathrm{H}_{48} \mathrm{O}_{5}$ (524), gelbes Ö1, UV-löschend (254 nm). $-\boldsymbol{R}_{\boldsymbol{f}}=0.65$ $\left(\mathrm{CH}_{2} \mathrm{Cl}_{2} / \mathrm{MeOH}\right.$ 95:5). - Anfärbeverhalten: Anisaldehyd (blau), Ehrlich (beige). $-\boldsymbol{R}_{\boldsymbol{t}}=24.6$ $\min (\mathrm{LC}-\mathrm{MS}) .-{ }^{1} \mathbf{H}-\mathbf{N M R}\left(\mathrm{CDCl}_{3}, 600 \mathrm{MHz}\right): \delta=5.07\left(\mathrm{t},{ }^{3} J=6.9 \mathrm{~Hz}, 2 \mathrm{H}, 16-, 20-\mathrm{H}\right), 5.04$ $\left(\mathrm{t},{ }^{3} J=6.9 \mathrm{~Hz}, 1 \mathrm{H}, 12-\mathrm{H}\right) 4.91\left(\mathrm{t},{ }^{3} J=6.9 \mathrm{~Hz}, 1 \mathrm{H}, 8-\mathrm{H}\right), 3.97$ (s, 3H, 5-OCH 3 ), 3.96 (s, 3H, 6$\left.\mathrm{OCH}_{3}\right), 3.16\left(\mathrm{~d},{ }^{3} J=6.9 \mathrm{~Hz}, 2 \mathrm{H}, 7-\mathrm{H}\right), 2.35$ (t, $\left.{ }^{3} J=7.4 \mathrm{~Hz}, 2 \mathrm{H}, 24-\mathrm{H}\right), 2.10$ (s, 3H, 26-H), 2.06-2.01 (m, 6H, 11-, 15-, 19-H), 1.99 (s, 3H, 2-H), 1.96-1.92 (m, 8H, 10-, 14-, 18-, 22-H), 1.72 (s, 3H, 9-CH $), 1.64$ (quintett, $\left.{ }^{3} J=7.4 \mathrm{~Hz}, 2 \mathrm{H}, 23-\mathrm{H}\right), 1.55$ (s, 9H, 13-, 17-, 21-H). ${ }^{13}$ C-NMR $\left(\mathrm{CDCl}_{3}, 150.8 \mathrm{MHz}\right): \delta=209.2\left(\mathrm{C}_{\mathrm{q}}, \mathrm{C}-25\right), 184.8\left(\mathrm{C}_{\mathrm{q}}, \mathrm{C}-1\right), 183.9\left(\mathrm{C}_{\mathrm{q}}, \mathrm{C}-4\right), 144.3$ $\left(\mathrm{C}_{\mathrm{q}}, \mathrm{C}-5\right), 144.2\left(\mathrm{C}_{\mathrm{q}}, \mathrm{C}-6\right), 141.7\left(\mathrm{C}_{\mathrm{q}}, \mathrm{C}-3\right), 138.9\left(\mathrm{C}_{\mathrm{q}}, \mathrm{C}-2\right), 137.6\left(\mathrm{C}_{\mathrm{q}}, \mathrm{C}-9\right), 135.2\left(\mathrm{C}_{\mathrm{q}}, \mathrm{C}-13\right)$, $134.9\left(\mathrm{C}_{\mathrm{q}}, \mathrm{C}-17\right), 134.1$ ( $\left.\mathrm{C}_{\mathrm{q}}, \mathrm{C}-21\right), 125.1$ (CH, C-20), 124.2 (CH, C-16), 123.9 (CH, C-12), $118.8(\mathrm{CH}, \mathrm{C}-8), 61.1\left(\mathrm{CH}_{3}, 5-\mathrm{OCH}_{3}\right), 61.1\left(\mathrm{CH}_{3}, 6-\mathrm{OCH}_{3}\right), 43.0\left(\mathrm{CH}_{2}, \mathrm{C}-24\right), 39.7\left(\mathrm{CH}_{2}, \mathrm{C}-\right.$ 10), $39.7\left(\mathrm{CH}_{2}, \mathrm{C}-14\right), 39.7\left(\mathrm{CH}_{2}, \mathrm{C}-18\right), 38.9\left(\mathrm{CH}_{2}, \mathrm{C}-22\right), 30.0\left(\mathrm{CH}_{3}, \mathrm{C}-26\right), 26.7\left(\mathrm{CH}_{2}, \mathrm{C}-\right.$ 11), $26.6\left(\mathrm{CH}_{2}, \mathrm{C}-15\right), 26.5\left(\mathrm{CH}_{2}, \mathrm{C}-19\right), 25.3\left(\mathrm{CH}_{2}, \mathrm{C}-7\right), 21.8\left(\mathrm{CH}_{2}, \mathrm{C}-23\right), 16.3\left(\mathrm{CH}_{3}, 9-\right.$ $\left.\mathrm{CH}_{3}\right), 16.0\left(\mathrm{CH}_{3}, 13-\mathrm{CH}_{3}\right), 16.0\left(\mathrm{CH}_{3}, 17-\mathrm{CH}_{3}\right), 15.7\left(\mathrm{CH}_{3}, 21-\mathrm{CH}_{3}\right), 11.9\left(\mathrm{CH}_{3}, 2-\mathrm{CH}_{3}\right)$. $(+)$-ESI-MS: $m / z(\%)=1071[2 \mathrm{M}+\mathrm{Na}]^{+}(100), 547[\mathrm{M}+\mathrm{Na}]^{+}(25) .-$ HRMS ber. für $\mathrm{C}_{33} \mathrm{H}_{48} \mathrm{O}_{5}$ : 524.35016, gef.: 524.35006. - UV (MeOH): $\lambda_{\max }(\lg \varepsilon)=275$ (3.90) nm. - IR $(\mathrm{KBr}): \widetilde{v}\left(\mathrm{~cm}^{-1}\right)=2929,2856,1718,1652,1612,1457,1385,1288,1265,1205,1155,1104$, 1027.

Ubichinon KQ2 (30): $\mathrm{C}_{18} \mathrm{H}_{24} \mathrm{O}_{5}$ (320), gelbes Ö1,

UV-löschend $(254 \mathrm{~nm}) . \quad-\quad \boldsymbol{R}_{\boldsymbol{f}}=0.56$ $\left(\mathrm{CH}_{2} \mathrm{Cl}_{2} / \mathrm{MeOH}\right.$ 95:5). - Anfärbeverhalten: Anisaldehyd (grün), Ehrlich (beige). $\boldsymbol{R}_{t}=17.7 \mathrm{~min}$ (LC-MS). - ${ }^{1} \mathbf{H}-\mathbf{N M R}\left(\mathrm{CDCl}_{3}, 600 \mathrm{MHz}\right): \delta=$<smiles>COC1=C(OC)C(=O)C(C/C=C(\C)CCC(C)=O)=C(C)C1=O</smiles>
$4.91\left(\mathrm{t},{ }^{3} \mathrm{~J}=6.9 \mathrm{~Hz}, 1 \mathrm{H}, 8-\mathrm{H}\right), 3.98(\mathrm{~s}, 3 \mathrm{H}, 5-$ $\left.\mathrm{OCH}_{3}\right), 3.96\left(\mathrm{~s}, 3 \mathrm{H}, 6-\mathrm{OCH}_{3}\right), 3.16\left(\mathrm{~d},{ }^{3} \mathrm{~J}=6.9 \mathrm{~Hz}, 2 \mathrm{H}, 7-\mathrm{H}\right), 2.33\left(\mathrm{t},{ }^{3} J=7.5 \mathrm{~Hz}, 2 \mathrm{H}, 12-\mathrm{H}\right)$, 2.09 (s, 3H, 14-H), 1.99 (s, 3H, 2- $\left.\mathrm{CH}_{3}\right), 1.93\left(\mathrm{t},{ }^{3} \mathrm{~J}=7.5 \mathrm{~Hz}, 2 \mathrm{H}, 10-\mathrm{H}\right), 1.70$ (s, 3H, 9- $\left.\mathrm{CH}_{3}\right)$, 1.64 (quintett, $\left.{ }^{3} J=7.5 \mathrm{~Hz}, 2 \mathrm{H}, 11-\mathrm{H}\right) .-{ }^{13} \mathbf{C}-\mathbf{N M R}\left(\mathrm{CDCl}_{3}, 150.8 \mathrm{MHz}\right): \delta=208.9\left(\mathrm{C}_{\mathrm{q}}, \mathrm{C}-\right.$ 13), $184.7\left(\mathrm{C}_{\mathrm{q}}, \mathrm{C}-1\right), 183.9\left(\mathrm{C}_{\mathrm{q}}, \mathrm{C}-4\right), 144.4\left(\mathrm{C}_{\mathrm{q}}, \mathrm{C}-5\right), 144.2\left(\mathrm{C}_{\mathrm{q}}, \mathrm{C}-6\right), 141.5\left(\mathrm{C}_{\mathrm{q}}, \mathrm{C}-2\right), 138.9$ $\left(\mathrm{C}_{\mathrm{q}}, \mathrm{C}-3\right), 136.9\left(\mathrm{C}_{\mathrm{q}}, \mathrm{C}-9\right), 119.7(\mathrm{CH}, \mathrm{C}-8), 61.2\left(\mathrm{CH}_{3}, 5-\mathrm{OCH}_{3}\right), 61.2\left(\mathrm{CH}_{3}, 6-\mathrm{OCH}_{3}\right), 42.9$ 
$\left(\mathrm{CH}_{2}, \mathrm{C}-12\right), 38.9\left(\mathrm{CH}_{2}, \mathrm{C}-10\right), 30.0\left(\mathrm{CH}_{3}, \mathrm{C}-14\right), 25.3\left(\mathrm{CH}_{2}, \mathrm{C}-7\right), 21.7\left(\mathrm{CH}_{2}, \mathrm{C}-11\right), 16.1$ (9$\left.\mathrm{CH}_{3}\right), 12.0\left(2-\mathrm{CH}_{3}\right) .-(+)$-ESI-MS: $m / z(\%)=663[2 \mathrm{M}+\mathrm{Na}]^{+}(100), 343[\mathrm{M}+\mathrm{Na}]^{+}(51) .-$ HRMS ber. für $\mathrm{C}_{18} \mathrm{H}_{24} \mathrm{O}_{5}$ : 320.16237, gef.: 320.16242. - UV (MeOH): $\lambda_{\max }(\lg \varepsilon)=209$ (4.04), 274 (3.98), 394 (2.52) nm. - IR (KBr): $\widetilde{v}\left(\mathrm{~cm}^{-1}\right)=2931,2855,1716,1651,1612$, $1457,1385,1287,1266,1205,1157,1102,1018,946$.

Norharman (31a): $\mathrm{C}_{11} \mathrm{H}_{8} \mathrm{~N}_{2}$ (168), weißer Feststoff, UVlöschend (254 nm), fluoreszierend (366 nm). $-\boldsymbol{R}_{\boldsymbol{f}}=0.37$ $\left(\mathrm{CH}_{2} \mathrm{Cl}_{2} / \mathrm{MeOH} 9: 1\right)$. - Keine Anfärbung mit Anisaldehyd und Ehrlichs Reagenz. $-\boldsymbol{R}_{\boldsymbol{t}}=5.9 \mathrm{~min}(\mathrm{LC}-\mathrm{MS}) .-{ }^{1} \mathbf{H}-\mathbf{N M R}\left(\mathrm{D}_{6^{-}}\right.$

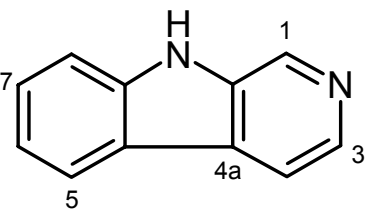
Aceton, $600 \mathrm{MHz}): \delta=10.78(\mathrm{br}, 1 \mathrm{H}, 9-\mathrm{NH}), 8.90$ (s, 1H, 1-H), 8.35 (d, ${ }^{3} J=6.1 \mathrm{~Hz}, 1 \mathrm{H}, 3-$ H), $8.22\left(\mathrm{~d},{ }^{3} J=6.1 \mathrm{~Hz}, 1 \mathrm{H}, 4-\mathrm{H}\right), 8.05\left(\mathrm{~d},{ }^{3} J=7.8 \mathrm{~Hz}, 1 \mathrm{H}, 5-\mathrm{H}\right), 7.62\left(\mathrm{~d},{ }^{3} J=8.2 \mathrm{~Hz}, 1 \mathrm{H}, 8-\right.$ H), $7.53\left(\mathrm{t},{ }^{3} J=7.9 \mathrm{~Hz}, 1 \mathrm{H}, 7-\mathrm{H}\right), 7.25\left(\mathrm{t},{ }^{3} J=8.0 \mathrm{~Hz}, 1 \mathrm{H}, 6-\mathrm{H}\right)$. - EI-MS $(70 \mathrm{eV}): \mathrm{m} / z(\%)=$ $168\left(\left([\mathrm{M}]^{++}, 100\right), 140(14)\right.$.

Harman (31b): $\mathrm{C}_{12} \mathrm{H}_{10} \mathrm{~N}_{2}$ (182), weißer Feststoff, UV-löschend $(254 \mathrm{~nm})$, fluoreszierend (366 nm). $-\boldsymbol{R}_{\boldsymbol{f}}=0.35\left(\mathrm{CH}_{2} \mathrm{Cl}_{2} / \mathrm{MeOH}\right.$ 9:1). - Keine Anfärbung mit Anisaldehyd und Ehrlichs Reagenz. $-\boldsymbol{R}_{\boldsymbol{t}}=6.7 \mathrm{~min}(\mathrm{LC}-\mathrm{MS}) .-{ }^{1} \mathbf{H}-\mathbf{N M R}\left(\mathrm{D}_{6}\right.$-Aceton, $\left.300 \mathrm{MHz}\right): \delta=$

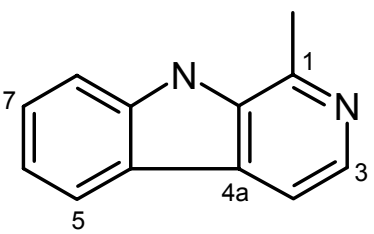
10.72 (br, 1H, 9-NH), 8.24 (d, $\left.{ }^{3} J=6.2 \mathrm{~Hz}, 1 \mathrm{H}, 3-\mathrm{H}\right), 8.18\left(\mathrm{~d},{ }^{3} J=\right.$ $6.2 \mathrm{~Hz}, 1 \mathrm{H}, 4-\mathrm{H}), 7.89\left(\mathrm{~d},{ }^{3} J=7.8 \mathrm{~Hz}, 1 \mathrm{H}, 5-\mathrm{H}\right), 7.60\left(\mathrm{~d},{ }^{3} J=8.2 \mathrm{~Hz}, 1 \mathrm{H}, 8-\mathrm{H}\right), 7.51\left(\mathrm{t},{ }^{3} J=\right.$ $7.9 \mathrm{~Hz}, 1 \mathrm{H}, 7-\mathrm{H}), 7.23\left(\mathrm{t},{ }^{3} \mathrm{~J}=8.0 \mathrm{~Hz}, 1 \mathrm{H}, 6-\mathrm{H}\right), 2.77\left(\mathrm{~s}, 3 \mathrm{H}, 1-\mathrm{CH}_{3}\right)$. - EI-MS $(70 \mathrm{eV}): \mathrm{m} / z$ $(\%)=182\left(\left([\mathrm{M}]^{\circ+}, 100\right), 154(14)\right.$.

Glutarsäure (32a): $\mathrm{C}_{5} \mathrm{H}_{8} \mathrm{O}_{4}$ (132), farbloser Feststoff, nicht UV-aktiv. $-\boldsymbol{R}_{\boldsymbol{f}}=0.25\left(\mathrm{CH}_{2} \mathrm{Cl}_{2} / \mathrm{MeOH} 95: 5\right)$. - Keine Anfärbung mit Anisaldehyd und Ehrlichs Reagenz. - ${ }^{1}$ H-NMR $\left(\mathrm{D}_{6^{-}}\right.$<smiles>O=C(O)CCCC(=O)O</smiles>
Aceton, $300 \mathrm{MHz}$ ): $\delta=2.37\left(\mathrm{t},{ }^{3} J=6.8 \mathrm{~Hz}, 4 \mathrm{H}, 2-\mathrm{H}, 4-\mathrm{H}\right.$ ), 1.85 (quintet, ${ }^{3} J=6.8 \mathrm{~Hz}, 2 \mathrm{H}, 3-$ H). - (+)-ESI-MS: $m / z(\%)=155[\mathrm{M}+\mathrm{Na}]^{+}(98)$.

Glutarsäuremonomethylester (32b): $\mathrm{C}_{6} \mathrm{H}_{7} \mathrm{O}_{4}$ (128), farblose Flüssigkeit, nicht UV-aktiv. $-\boldsymbol{R}_{\boldsymbol{f}}=0.42\left(\mathrm{CH}_{2} \mathrm{Cl}_{2} / \mathrm{MeOH}\right.$ 95:5). - Keine Anfärbung mit Anisaldehyd und Ehrlichs Rea-<smiles>COC(=O)CCCC(=O)O</smilesgenz. $-\boldsymbol{R}_{\boldsymbol{t}}=10.6 \mathrm{~min}(\mathrm{LC}-\mathrm{MS}) .{ }^{1}{ }^{1} \mathbf{H}-\mathbf{N M R}\left(\mathrm{CDCl}_{3}, 300 \mathrm{MHz}\right): \delta=\sim 10$ (br, $\left.1 \mathrm{H}, \mathrm{OH}\right), 3.69$ (s, 3H, $\left.\mathrm{OCH}_{3}\right), 2.44\left(\mathrm{t},{ }^{3} J=6.7 \mathrm{~Hz}, 2 \mathrm{H}, 4-\mathrm{H}\right), 2.42\left(\mathrm{t},{ }^{3} J=6.7 \mathrm{~Hz}, 2 \mathrm{H}, 2-\mathrm{H}\right), 1.96$ (quintet, ${ }^{3} J$ 
$=6.7 \mathrm{~Hz}, 2 \mathrm{H}, 3-\mathrm{H}) .-\mathbf{E I}-\mathbf{M S}(70 \mathrm{eV}): m / z(\%)=128\left(\left([\mathrm{M}]^{\circ+}, 24\right), 115(100), 114(58), 100\right.$ (83), 87 (89), 86 (53), 74 (30), 59 (44), 55 (60), 45 (58), 43 (90), 42 (40), 41 (47).

Indolcarbonsäure (33): $\mathrm{C}_{9} \mathrm{H}_{7} \mathrm{NO}_{2}$ (161), farbloses Öl, UV-löschend. $-\boldsymbol{R}_{\boldsymbol{f}}=0.44\left(\mathrm{CH}_{2} \mathrm{Cl}_{2} / \mathrm{MeOH}\right.$ 9:1). - Anfärbeverhalten: Anisaldehyd (orange), Ehrlich (violett). $-\boldsymbol{R}_{\boldsymbol{t}}=11.4 \mathrm{~min}$ (LC-MS). $-{ }^{\mathbf{1}} \mathbf{H}-\mathbf{N M R}$ $\left(\mathrm{CD}_{3} \mathrm{OD}, 300 \mathrm{MHz}\right): \delta=8.09\left(\mathrm{~d},{ }^{3} J=8.1 \mathrm{~Hz}, 1 \mathrm{H}, 4-\mathrm{H}\right), 7.88(\mathrm{~s}, 1 \mathrm{H}$,

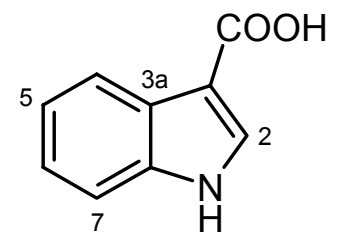
2-H), $7.40\left(\mathrm{~d},{ }^{3} J=8.1 \mathrm{~Hz}, 1 \mathrm{H}, 7-\mathrm{H}\right), 7.16\left(\mathrm{t},{ }^{3} J=8.1 \mathrm{~Hz}, 1 \mathrm{H}, 6-\mathrm{H}\right), 7.13\left(\mathrm{t},{ }^{3} J=8.1 \mathrm{~Hz}, 1 \mathrm{H}, 5-\right.$ H). - EI-MS (70 eV): $m / z(\%)=161\left(\left([\mathrm{M}]^{\bullet+}, 100\right), 144(91), 116(25), 89(34)\right.$.

3-Hydroxyacetylindol (34): $\mathrm{C}_{10} \mathrm{H}_{9} \mathrm{NO}_{2}$ (175), weißer Feststoff, UV-löschend. $-\boldsymbol{R}_{\boldsymbol{f}}=0.28\left(\mathrm{CH}_{2} \mathrm{Cl}_{2} / \mathrm{MeOH} 95: 5\right)$. - Anfärbeverhalten: Anisaldehyd (braun), Ehrlich (hellrot). $-\boldsymbol{R}_{\boldsymbol{t}}=10.1 \mathrm{~min}$ (LCMS). $-{ }^{1} \mathbf{H}-\mathbf{N M R}\left(\mathrm{CD}_{3} \mathrm{OD}, 300 \mathrm{MHz}\right): \delta=8.22$ (m, 1H, 4-H), 8.19 (s, 1H, 2-H), 7.44 (m, 1H, 7-H), 7.22 (m, 2H, 5-H, 6-H), 4.72 (s,<smiles>O=C(CO)c1c[nH]c2ccccc12</smiles>
2H, 9-H). - EI-MS $(70 \mathrm{eV}): m / z(\%)=175\left(\left([\mathrm{M}]^{\bullet+}, 24\right), 144(100)\right.$, $116(18), 89(12)$.

Uracil (35): $\mathrm{C}_{4} \mathrm{H}_{4} \mathrm{~N}_{2} \mathrm{O}_{2}$ (112), weißer Feststoff, UV-löschend. $-\boldsymbol{R}_{\boldsymbol{f}}=0.25$ $\left(\mathrm{CH}_{2} \mathrm{Cl}_{2} / \mathrm{MeOH}\right.$ 9:1). - Keine Anfärbung mit Anisaldehyd und Ehrlichs Reagenz. - ${ }^{1}$ H-NMR ([D $\left.{ }_{6}\right]$ DMSO, $\left.300 \mathrm{MHz}\right): \delta=10.95$ (br, 1H, 2-NH), 10.77 (br, 1H, 4-NH), 7.38 (d, $\left.{ }^{3} J=8.2 \mathrm{~Hz}, 1 \mathrm{H}, 5-\mathrm{H}\right), 5.44$ (d, ${ }^{3} J=8.2 \mathrm{~Hz}$, 1H, 6-H). - EI-MS (70 eV): $m / z(\%)=112\left(\left([\mathrm{M}]^{++}, 26\right), 69(47), 42(100)\right.$.<smiles></smiles>

p-Hydroxy-benzoesäure (36): $\mathrm{C}_{7} \mathrm{H}_{6} \mathrm{O}_{3}$ (138), weißer Feststoff, UVlöschend. $-\boldsymbol{R}_{\boldsymbol{f}}=0.49\left(\mathrm{CH}_{2} \mathrm{Cl}_{2} / \mathrm{MeOH}\right.$ 9:1). - Anfärbeverhalten: Ehrlich (violett). $-\boldsymbol{R}_{\boldsymbol{t}}=7.7$ (LC-MS). - ${ }^{1} \mathbf{H}-\mathbf{N M R}\left(\left[\mathrm{D}_{6}\right] \mathrm{DMSO}, 300 \mathrm{MHz}\right): \delta=7.86$ $\left(\mathrm{d},{ }^{3} J=8.6 \mathrm{~Hz}, 2 \mathrm{H}, 3-\mathrm{H}, 5-\mathrm{H}\right), 6.80$ (d, $\left.{ }^{3} J=8.6 \mathrm{~Hz}, 2 \mathrm{H}, 2-\mathrm{H}, 6-\mathrm{H}\right)$. - EI-MS $(70 \mathrm{eV}): m / z(\%)=138\left(\left([\mathrm{M}]^{\circ+}, 54\right), 121(100), 93(22), 65(19)\right.$.<smiles>O=C(O)c1ccc(O)cc1</smiles>

p-Hydroxyphenylethanol (37): $\mathrm{C}_{8} \mathrm{H}_{10} \mathrm{O}_{2}$ (138), weißer Feststoff, UVlöschend. $-\boldsymbol{R}_{\boldsymbol{f}}=0.44\left(\mathrm{CH}_{2} \mathrm{Cl}_{2} / \mathrm{MeOH} 9: 1\right)$. - Anfärbeverhalten: Anisaldehyd (violett). $-{ }^{1} \mathbf{H}-\mathbf{N M R}$ NMR $\left(\mathrm{CDCl}_{3}, 300 \mathrm{MHz}\right): \delta=7.10\left(\mathrm{~d},{ }^{3} J=\right.$ $8.5 \mathrm{~Hz}, 2 \mathrm{H}, 2^{\prime}-\mathrm{H}^{\prime}$ 6'-H), 6.78 (d, '3 $\mathrm{J}=8.5 \mathrm{~Hz}, 2 \mathrm{H}, 3^{\prime}$-H, 5'-H), 4.84 (br, $1 \mathrm{H}, \mathrm{OH}), 3.83$ (t, $\left.{ }^{3} \mathrm{~J}=8.0 \mathrm{~Hz}, 2 \mathrm{H}, 1-\mathrm{H}\right), 2.81$ (t, $\left.{ }^{3} \mathrm{~J}=8.0 \mathrm{~Hz}, 2 \mathrm{H}, 2-\mathrm{H}\right)$. -<smiles>OCCc1ccc(O)cc1</smiles> 
EI-MS $(70 \mathrm{eV}): m / z(\%)=138\left(\left([\mathrm{M}]^{\circ+}, 34\right), 107(100), 77(10)\right.$.

Vanillinsäure (38): $\mathrm{C}_{8} \mathrm{H}_{8} \mathrm{O}_{4}$ (168), weißer Feststoff, UV-löschend. - $\boldsymbol{R}_{\boldsymbol{f}}$ $=0.42\left(\mathrm{CH}_{2} \mathrm{Cl}_{2} / \mathrm{MeOH}\right.$ 9:1). $\quad-$ Anfärbeverhalten: Anisaldehyd (hellblau). $-\boldsymbol{R}_{\boldsymbol{t}}=8.3$ (LC-MS).. $-{ }^{1}$ H-NMR $\left(\mathrm{CD}_{3} \mathrm{OD}, 300 \mathrm{MHz}\right): \delta=$ 7.55 (m, 2H, 2-H, 6-H), $6.82\left(\mathrm{~d},{ }^{3} J=8.0 \mathrm{~Hz}, 1 \mathrm{H}, 5-\mathrm{H}\right), 3.89$ (s, 3H, $\left.\mathrm{OCH}_{3}\right)$. - EI-MS $(70 \mathrm{eV}): m / z(\%)=168\left(\left([\mathrm{M}]^{\circ+}, 100\right), 153(61), 125\right.$<smiles>COc1cc(C(=O)O)ccc1O</smiles>
(22), 97 (36), 51 (19).

\section{4-Hydroxy-3-methoxy-phenyl-propionsäure (39):}

$\mathrm{C}_{10} \mathrm{H}_{12} \mathrm{O}_{4}$ (196), weißer Feststoff, schwach UV-löschend.

- $\boldsymbol{R}_{\boldsymbol{f}}=0.44\left(\mathrm{CH}_{2} \mathrm{Cl}_{2} / \mathrm{MeOH}\right.$ 9:1). - Anfärbeverhalten: Anisaldehyd (hellrot). $-{ }^{1} \mathbf{H}$-NMR $\left(\mathrm{CDCl}_{3}, 300 \mathrm{MHz}\right): \delta=$<smiles>COc1cc(CCC(=O)O)ccc1O</smiles>
$6.84\left(\mathrm{~d}, 1 \mathrm{H},{ }^{3} \mathrm{~J}=8.5 \mathrm{~Hz}, 5^{\prime}-\mathrm{H}\right), 6.71$ (s, 1H, 2'-H), 6.70 (d, ${ }^{3} J=8.5 \mathrm{~Hz}, 1 \mathrm{H}, 6$ '-H), 5.50 (br, $\left.1 \mathrm{H}, \mathrm{OH}\right), 3.87$ (s, 3H, $\left.\mathrm{OCH}_{3}\right), 2.89$ (t, $\left.{ }^{3} J=7.1 \mathrm{~Hz}, 2 \mathrm{H}, \mathrm{H}-2\right)$, $2.66\left(\mathrm{t},{ }^{3} J=7.1 \mathrm{~Hz}, 2 \mathrm{H}, \mathrm{H}-3\right)$. - EI-MS $(70 \mathrm{eV}): m / z(\%)=196\left(\left([\mathrm{M}]^{++}, 37\right), 137(100)\right.$.

\subsection{Salegentibacter holothuriorum T436}

Der aus mehrere Jahre altem arktischen Eis isolierte Stamm T436 bildete auf Agar-Platten beige Kolonien und färbte den Agar intensiv gelb.

\subsubsection{Primärscreening}

Der Rohextrakt des Stammes T436 zeigte im Dünnschichtchromatogramm $\left(\mathrm{CH}_{2} \mathrm{Cl}_{2} / \mathrm{MeOH}\right.$ 9:1) eine Reihe gelber und oranger Zonen auf, die sich sowohl im polaren als auch im unpolaren Bereich befanden. Besprühen mit Anisaldehyd und Ehrlichs Reagenz sowie Zugabe von $2 \mathrm{~N} \mathrm{NaOH}$ führten zu keiner Farbänderung. Im biologischen Screening (300 $\mu \mathrm{g} /$ Plättchen) zeigte der Rohextrakt des Stammes folgende Aktivitäten: 
Tabelle 9 Aktivität des Rohextraktes von Salegentibacter holothuriorum T436 im biologischen Screening.

\begin{tabular}{|l|c|}
\hline \multicolumn{1}{|c|}{ Testkeim } & Hemmhof $\varnothing[\mathrm{mm}]$ \\
\hline \hline Bacillus brevis (ATCC 9999) & 17 \\
\hline Bacillus subtilis (ATCC 6633) & 17 \\
\hline Nematospora coryli (ATCC 10647) & 19 \\
\hline Micrococcus luteus (ATCC 381) & 15 \\
\hline Mucor miehei (Tü 284) & 0 \\
\hline Paecilomyces variotii (ETH 114646) & 0 \\
\hline
\end{tabular}

\subsubsection{Kultivierung und Aufarbeitung}

Die Kultivierung des Stammes T436 wurde am Institut für Biotechnologie und WirkstoffForschung (Kaiserslautern) in einem 20 l-Fermenter unter Verwendung des B-Mediums durchgeführt. Die Fermentation erfolgte bei einem Belüftungsdruck von $31 /$ min und einer Rührgeschwindigkeit von $121 \mathrm{Upm}$ für 5 Tage bei $22^{\circ} \mathrm{C}$.

\subsubsection{Isolierung und Charakterisierung der Metaboliten}

Der Rohextrakt wurde durch Säulenchromatographie an Kieselgel mittels eines Stufengradienten aus Dichlormethan/Methanol $\left(\mathrm{CH}_{2} \mathrm{Cl}_{2}, \mathrm{CH}_{2} \mathrm{Cl}_{2} / \mathrm{MeOH}\right.$ 99:1, $\mathrm{CH}_{2} \mathrm{Cl}_{2} / \mathrm{MeOH}$ 98:2, $\mathrm{CH}_{2} \mathrm{Cl}_{2} / \mathrm{MeOH}$ 97:3, $\mathrm{CH}_{2} \mathrm{Cl}_{2} / \mathrm{MeOH}$ 96:4, $\mathrm{CH}_{2} \mathrm{Cl}_{2} / \mathrm{MeOH}$ 95:5, $\mathrm{CH}_{2} \mathrm{Cl}_{2} / \mathrm{MeOH}$ 9:1, $\mathrm{CH}_{2} \mathrm{Cl}_{2} /$ $\mathrm{MeOH}$ 4:1) getrennt. Die erhaltenen Fraktionen wurden durch weitere Chromatographie an Sephadex ${ }^{\circledR} \mathrm{LH}-20\left(\mathrm{CH}_{2} \mathrm{Cl}_{2} / \mathrm{MeOH} 3: 2\right.$ und $\left.\mathrm{MeOH}\right)$ gereinigt. Nachfolgende Flashchromatographie an Kieselgel $\left(\mathrm{CH}_{2} \mathrm{Cl}_{2} / \mathrm{MeOH}\right)$ ergab $0.4 \mathrm{mg}$-Nitrophenol (40), $1.8 \mathrm{mg}$ 4-Hydroxy-3nitro-benzoesäure (41), 3.0 mg 4-Hydroxy-3-nitrophenyl-essigsäure (42), 1.7 mg 4-Hydroxy3-nitrophenyl-propionsäure $\quad \mathbf{( 4 3 )}, \quad 1.7 \mathrm{mg}$ 4-Hydroxy-3-nitrophenyl-essigsäuremethylester (44), 2.6 mg 2-(4-Hydroxy-3-nitrophenyl)ethanol (45), 7.1 mg $N$-(4-Hydroxy-3-nitrophenylethyl)acetamide (46), 0.4 mg 2-Nitro-4-(2'-nitroethenyl)phenol (47), 0.4 mg 3,5-Dinitro-4hydroxy-phenyl-essigsäuremethylester (48), $1.1 \mathrm{mg}$ 3,5-Dinitro-4-hydroxy-phenyl-propionsäure (49), 0.6 mg 3,5-Dinitro-4-hydroxy-phenyl-ethylchlorid (50), 0.7 mg 5-Dinitro-4hydroxy-phenyl-2-chlor-propionsäuremethylester (51), $2.7 \mathrm{mg} \quad \mathrm{N}$-(3,5-Dinitro-4-hydroxyphenylethyl)-acetamid (52), 0.4 mg 2,6-Dinitro-4-(2'-nitroethenyl)phenol (53), 6.1 mg Pyri- 
culamide (55), $1.1 \mathrm{mg}$ 7-Hydroxy-3-(4-hydroxy-2-nitrophenyl)-4H-1-benzopyran-4-on (3'Nitro-daidzein) (56), $3.9 \mathrm{mg} \quad$ 5,7-Dihydroxy-3-(3,5-dinitro-4-hydroxy-phenyl)-4H-1benzopyran-4-on (3',5'-Dinitro-genistein) (57), $0.8 \mathrm{mg}$ 2-Nitro- $N_{\beta}$-acetyltryptamin (58), 6Nitro- $N_{\beta}$-acetyltryptamin (59), 7-Nitro- $N_{\beta}$-acetyltryptamin (60), $0.4 \mathrm{mg}$ Salegentipyrrol A (61), 0.9 mg Salegentipyrrol B (64), $1.8 \mathrm{mg}$ 4,5-Dinitro-3-methoxy-phenol (65), $1.2 \mathrm{mg}$ 4,6Dinitro-2-methoxy-phenol (66), $2.1 \mathrm{mg}$ 2,6-Dimethoxy-1,4-benzoquinon (67), $1.1 \mathrm{mg} 3$ Indolyl-ethyl-methylether (68), $4.7 \mathrm{mg}$ Thymin (69), $4.2 \mathrm{mg}$ Uracil (35) und $2.2 \mathrm{mg}$ pHyxdroxybenzoesäure (36).

p-Nitrophenol (40): $\mathrm{C}_{6} \mathrm{H}_{5} \mathrm{NO}_{3}$ (139), hellgelber Feststoff, UV-löschend (254 $\mathrm{nm}) .-\boldsymbol{R}_{\boldsymbol{f}}=0.60\left(\mathrm{CH}_{2} \mathrm{Cl}_{2} / \mathrm{MeOH} 9: 1\right) .-\boldsymbol{R}_{\boldsymbol{t}}=12.1 \min (\mathrm{LC}-\mathrm{MS}) .-{ }^{1} \mathbf{H}-\mathrm{NMR}$ $\left(\mathrm{CD}_{3} \mathrm{OD}, 600 \mathrm{MHz}\right): \delta=8.12\left(\mathrm{~d},{ }^{3} J=9.6 \mathrm{~Hz}, 2 \mathrm{H}, 2-\mathrm{H}, 6-\mathrm{H}\right), 6.88\left(\mathrm{~d},{ }^{3} J=9.6\right.$ Hz, 2H, 3-H, 5-H). - (-)-APCI: $m / z=138[\mathrm{M}-\mathrm{H}]^{-}$. - UV (MeOH): $\lambda_{\max }(\lg \varepsilon)=$ 223 (4.03), 309 (4.07) nm. - IR (KBr): $\widetilde{v}\left(\mathrm{~cm}^{-1}\right)=2928,2360,2340,1617$,<smiles>O=[N+]([O-])c1ccc(O)cc1</smiles>
$1595,1518,1500,1457,1385,1339,1290,1168,1112,852,755$.

4-Hydroxy-3-nitro-benzoesäure (41): $\mathrm{C}_{7} \mathrm{H}_{5} \mathrm{NO}_{5}$ (183), gelber Feststoff, UV-löschend (254 nm). $-\boldsymbol{R}_{\boldsymbol{f}}=0.25\left(\mathrm{CH}_{2} \mathrm{Cl}_{2} / \mathrm{MeOH} 9: 1\right) .-\boldsymbol{R}_{\boldsymbol{t}}=11.9 \mathrm{~min}$ (LC-MS). $-{ }^{1}$ H-NMR $\left(\mathrm{CD}_{3} \mathrm{OD}, 600 \mathrm{MHz}\right): \delta=8.66\left(\mathrm{~d},{ }^{4} J=1.8 \mathrm{~Hz}, 1 \mathrm{H}\right.$, 2-H), $8.15\left(\mathrm{dd},{ }^{4} J=1.8 \mathrm{~Hz},{ }^{3} J=8.4 \mathrm{~Hz}, 1 \mathrm{H}, 6-\mathrm{H}\right), 7.13\left(\mathrm{~d},{ }^{3} J=8.4 \mathrm{~Hz}\right.$, 1H, 5-H). $-{ }^{13} \mathbf{C}-\mathbf{N M R}\left(\mathrm{CD}_{3} \mathrm{OD}, 150.8 \mathrm{MHz}\right): \delta=169.8(\mathrm{COOH}), 158.0$<smiles>O=C(O)c1ccc(O)c([N+](=O)[O-])c1</smiles>
$\left(\mathrm{C}_{\mathrm{q}}, \mathrm{C}-4\right), 137.9(\mathrm{CH}, \mathrm{C}-6), 135.5\left(\mathrm{C}_{\mathrm{q}}, \mathrm{C}-3\right), 127.8(\mathrm{CH}, \mathrm{C}-2), 126.9\left(\mathrm{C}_{\mathrm{q}}\right.$, C-1), 120.8 (CH, C-5). - EI-MS (70 eV): $m / z(\%)=183\left(\left([\mathrm{M}]^{\circ+}, 100\right), 167\right.$ (12), $166(18)$, 153 (16), 119 (22), 81 (23), 63 (61), 53 (28). - UV (MeOH): $\lambda_{\max }(\lg \varepsilon)=237$ (4.38), 340 (3.51) nm. - IR (KBr): $\widetilde{v}\left(\mathrm{~cm}^{-1}\right)=3307,2924,2853,1685,1626,1574,1541,1433,1338$, $1312,1257,1175,1112$.

4-Hydroxy-3-nitrophenyl-essigsäure (42): $\mathrm{C}_{8} \mathrm{H}_{7} \mathrm{NO}_{5}$ (197), gelber Feststoff, UV-löschend $(254 \mathrm{~nm}) . \quad-\boldsymbol{R}_{\boldsymbol{f}}=0.39$ $\left(\mathrm{CH}_{2} \mathrm{Cl}_{2} / \mathrm{MeOH}\right.$ 9:1). $-\boldsymbol{R}_{\boldsymbol{t}}=11.3 \mathrm{~min}$ (LC-MS). - ${ }^{\mathbf{1}} \mathbf{H}-\mathbf{N M R}$ $\left(\mathrm{CD}_{3} \mathrm{OD}, 300 \mathrm{MHz}\right): \delta=8.01\left(\mathrm{~d},{ }^{4} J=2.1 \mathrm{~Hz}, 1 \mathrm{H}, 2-\mathrm{H}\right), 7.54$<smiles>O=C(O)Cc1ccc(O)c([N+](=O)[O-])c1</smiles>
$\left(\mathrm{dd},{ }^{3} J=8.5 \mathrm{~Hz},{ }^{4} J=2.1 \mathrm{~Hz}, 1 \mathrm{H}, 6-\mathrm{H}\right), 7.10\left(\mathrm{~d},{ }^{3} J=8.5 \mathrm{~Hz}, 1 \mathrm{H}, 5-\mathrm{H}\right), 3.63$ (s, 2H, $\left.\mathrm{CH}_{2} \mathrm{COO}\right)$. - EI-MS $(70 \mathrm{eV}): m / z(\%)=197\left(\left([\mathrm{M}]^{\circ+}, 23\right), 152\right.$ (100), 106 (20), 77 (19), 51 (18). - UV $(\mathrm{MeOH}): \lambda_{\max }(\lg \varepsilon)=226(3.88), 274$ (3.84), 354 (3.52) nm. - IR $(\mathrm{KBr}): \widetilde{v}\left(\mathrm{~cm}^{-1}\right)=3432$, 2930, 1698, 1632, 1581, 1493, 1434, 1412, 1331, 1258, 1216, 1180. 


\section{4-Hydroxy-3-nitrophenyl-propionsäure (43):}

$\mathrm{C}_{9} \mathrm{H}_{9} \mathrm{NO}_{5}$ (211), gelber Feststoff, UV-löschend (254 nm). $\boldsymbol{R}_{\boldsymbol{f}}=0.82\left(\mathrm{CH}_{2} \mathrm{Cl}_{2} / \mathrm{MeOH} 9: 1\right) . \boldsymbol{R}_{\boldsymbol{t}}=11.4 \mathrm{~min}(\mathrm{LC}-\mathrm{MS}) .-$

${ }^{1} \mathrm{H}-\mathrm{NMR}\left(\mathrm{CDCl}_{3}, 300 \mathrm{MHz}\right): \delta=10.46(\mathrm{~s}, 1 \mathrm{H}, 4-\mathrm{OH})$, $7.93\left(\mathrm{~d},{ }^{4} J=2.1 \mathrm{~Hz}, 1 \mathrm{H}, 2-\mathrm{H}\right), 7.43\left(\mathrm{dd},{ }^{3} J=8.4 \mathrm{~Hz},{ }^{4} J=\right.$<smiles>O=C(O)CCc1ccc(O)c([N+](=O)[O-])c1</smiles>
$2.1 \mathrm{~Hz}, 1 \mathrm{H}, 6-\mathrm{H}), 7.08\left(\mathrm{~d},{ }^{3} J=8.4 \mathrm{~Hz}, 1 \mathrm{H}, 5-\mathrm{H}\right), 2.93\left(\mathrm{t},{ }^{3} \mathrm{~J}\right.$ $\left.=7.0 \mathrm{~Hz}, 2 \mathrm{H}, \mathrm{CH}_{2} \mathrm{CH}_{2} \mathrm{COO}\right), 2.67\left(\mathrm{t},{ }^{3} \mathrm{~J}=7.0 \mathrm{~Hz}, 2 \mathrm{H}, \mathrm{CH}_{2} \mathrm{CH}_{2} \mathrm{COO}\right)$. - EI-MS $(70 \mathrm{eV}): \mathrm{m} / z$ $(\%)=211\left(\left([\mathrm{M}]^{++}, 32\right), 193(46), 152(100), 147(38), 106(15)\right.$. - UV (MeOH): $\lambda_{\max }(\lg \varepsilon)=$ 223 (3.98), 276 (3.92), 358 (3.59) nm. - IR (KBr): $\widetilde{v}\left(\mathrm{~cm}^{-1}\right)=2925,1710,1631,1583,1539$, 1490, 1429, 1327, 1244, 1187.

\section{4-Hydroxy-3-nitrophenyl-essigsäuremethylester (44):}

$\mathrm{C}_{8} \mathrm{H}_{7} \mathrm{NO}_{5}$ (211), gelber Feststoff, UV-löschend (254 nm). $\boldsymbol{R}_{\boldsymbol{f}}=0.76\left(\mathrm{CH}_{2} \mathrm{Cl}_{2} / \mathrm{MeOH} 9: 1\right) .-\boldsymbol{R}_{\boldsymbol{t}}=13.6 \mathrm{~min}(\mathrm{LC}-\mathrm{MS}) .-$ ${ }^{1}$ H-NMR $\left(\mathrm{CD}_{3} \mathrm{OD}, 300 \mathrm{MHz}\right): \delta=7.99\left(\mathrm{~d},{ }^{4} \mathrm{~J}=2.4 \mathrm{~Hz}, 1 \mathrm{H}\right.$,<smiles>COC(=O)Cc1ccc(O)c([N+](=O)[O-])c1</smiles>
2-H), $7.52\left(\mathrm{dd},{ }^{3} J=8.7 \mathrm{~Hz},{ }^{4} J=2.4 \mathrm{~Hz}, 1 \mathrm{H}, 6-\mathrm{H}\right), 7.09$ (d, $\left.{ }^{3} J=8.7 \mathrm{~Hz}, 1 \mathrm{H}, 5-\mathrm{H}\right), 3.69$ (s, 3H, COOMe), $3.67\left(\mathrm{~s}, 2 \mathrm{H}, \mathrm{CH}_{2}\right)$. - EI-MS $(70 \mathrm{eV}): m / z(\%)=211\left(\left([\mathrm{M}]^{\circ+}, 16\right), 165(11), 152\right.$ (100), 135 (12), 106 (44), 77 (36), 59 (23), 51 (35). - UV (MeOH): $\lambda_{\max }(\lg \varepsilon)=220$ (4.22), 273 (4.00), 354 (3.68) nm. - IR (KBr): $\widetilde{v}\left(\mathrm{~cm}^{-1}\right)=3280,3083,1736,1634,1581,1537,1486$, $1430,1420,1356,1330,1307,1262,1172$.

2-(4-Hydroxy-3-nitrophenyl)ethanol (45): $\mathrm{C}_{8} \mathrm{H}_{9} \mathrm{NO}_{4}$ (183), gelber Feststoff, UV-löschend $(254 \mathrm{~nm}) . \quad-\boldsymbol{R}_{\boldsymbol{f}}=0.59$ $\left(\mathrm{CH}_{2} \mathrm{Cl}_{2} / \mathrm{MeOH}\right.$ 9:1). $-\boldsymbol{R}_{\boldsymbol{t}}=13.2 \mathrm{~min}$ (LC-MS). $-{ }^{1} \mathbf{H}-\mathbf{N M R}$ $\left(\mathrm{CDCl}_{3}, 300 \mathrm{MHz}\right): \delta=10.46(\mathrm{~s}, 1 \mathrm{H}-4-\mathrm{OH}), 7.96\left(\mathrm{~d},{ }^{4} J=2.2\right.$

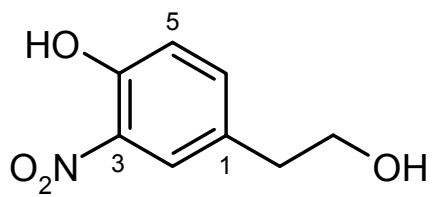
$\mathrm{Hz}, 1 \mathrm{H}, 2-\mathrm{H}), 7.46\left(\mathrm{dd},{ }^{3} J=8.5 \mathrm{~Hz},{ }^{4} J=2.2 \mathrm{~Hz}, 1 \mathrm{H}, 6-\mathrm{H}\right), 7.08\left(\mathrm{~d},{ }^{3} J=8.5 \mathrm{~Hz}, 1 \mathrm{H}, 5-\mathrm{H}\right)$, $3.86\left(\mathrm{t},{ }^{3} \mathrm{~J}=6.5 \mathrm{~Hz}, 2 \mathrm{H}, \mathrm{CH}_{2} \mathrm{CH}_{2} \mathrm{OH}\right), 2.84\left(\mathrm{t},{ }^{3} \mathrm{~J}=6.5 \mathrm{~Hz}, 2 \mathrm{H}, \mathrm{CH}_{2} \mathrm{CH}_{2} \mathrm{OH}\right) .-$ EI-MS $(70$ $\mathrm{eV}): m / z(\%)=183\left(\left([\mathrm{M}]^{\circ+}, 35\right), 152(100), 135(39), 106(31), 77\right.$ (20), 51 (9). - UV $(\mathrm{MeOH}): \lambda_{\max }(\lg \varepsilon)=221$ (4.12), 275 (3.93), 358 (3.59) nm. - IR (KBr): $\widetilde{v}\left(\mathrm{~cm}^{-1}\right)=3300$, 2929, 2847, 1632, 1586, 1488, 1427, 1326, 1253, 1179.

\section{$\mathrm{N}$-(4-Hydroxy-3-nitrophenylethyl)acetamide (46):}

$\mathrm{C}_{10} \mathrm{H}_{12} \mathrm{~N}_{2} \mathrm{O}_{4}$ (206), gelber Feststoff, UV-löschend (254 $\mathrm{nm}) .-\boldsymbol{R}_{\boldsymbol{f}}=0.51\left(\mathrm{CH}_{2} \mathrm{Cl}_{2} / \mathrm{MeOH} 9: 1\right) .-\boldsymbol{R}_{\boldsymbol{t}}=11.1 \mathrm{~min}$ (LC-MS). - ${ }^{1} \mathbf{H}-\mathbf{N M R}\left(\mathrm{CDCl}_{3}, 300 \mathrm{MHz}\right): \delta=10.44(\mathrm{~s}$,<smiles>CC(=O)NCCc1ccc(O)c([N+](=O)[O-])c1</smiles> 
$\mathrm{D}_{2} \mathrm{O}$-austauschbar, 1H, 4-OH), $7.89\left(\mathrm{~d},{ }^{4} \mathrm{~J}=2.1 \mathrm{~Hz}, 1 \mathrm{H}, 2-\mathrm{H}\right), 7.41\left(\mathrm{dd},{ }^{3} \mathrm{~J}=8.4 \mathrm{~Hz},{ }^{4} \mathrm{~J}=2.1\right.$ $\mathrm{Hz}, 1 \mathrm{H}, 5-\mathrm{H}), 7.08$ (d, $\left.{ }^{3} \mathrm{~J}=8.4 \mathrm{~Hz}, 1 \mathrm{H}, 6-\mathrm{H}\right), 5.72$ (br s, $\mathrm{D}_{2} \mathrm{O}$-austauschbar, 1H, NH), 3.46 (q, $\left.{ }^{3} J=6.9 \mathrm{~Hz}, 2 \mathrm{H}, \mathrm{CH}_{2} \mathrm{CH}_{2} \mathrm{NH}\right), 2.79\left(\mathrm{t},{ }^{3} \mathrm{~J}=6.9 \mathrm{~Hz}, 2 \mathrm{H}, \mathrm{CH}_{2} \mathrm{CH}_{2} \mathrm{NH}\right), 1.93$ (s, 3H, Ac). - EIMS (70 eV): $m / z(\%)=206\left(\left([\mathrm{M}]^{\circ+}, 60\right), 165\right.$ (100), 135 (23), 105 (19), 77 (15), 72 (18), 60 (17), 43 (41). - UV (MeOH): $\lambda_{\max }(\lg \varepsilon)=225$ (3.90), 275 (3.86), 357 (3.56) nm. - IR (KBr): $\widetilde{v}\left(\mathrm{~cm}^{-1}\right)=3079,2934,1634,1558,1532,1489,1424,1321,1291,1257,1171$.

2-Nitro-4-(2'-nitroethenyl)phenol (47): $\mathrm{C}_{8} \mathrm{H}_{6} \mathrm{~N}_{2} \mathrm{O}_{5}$ (210), gelber Feststoff, UV-löschend $(254 \mathrm{~nm}) . \quad-\boldsymbol{R}_{\boldsymbol{f}}=0.83$ $\left(\mathrm{CH}_{2} \mathrm{Cl}_{2} / \mathrm{MeOH}\right.$ 9:1). $-\boldsymbol{R}_{\boldsymbol{t}}=13.7$ min (LC-MS). $-{ }^{\mathbf{1}} \mathbf{H}-\mathbf{N M R}$ $\left(\mathrm{CD}_{3} \mathrm{OD}, 300 \mathrm{MHz}\right): \delta=8.36\left(\mathrm{~d},{ }^{4} J=2.4 \mathrm{~Hz}, 1 \mathrm{H}, 2-\mathrm{H}\right), 8.05$

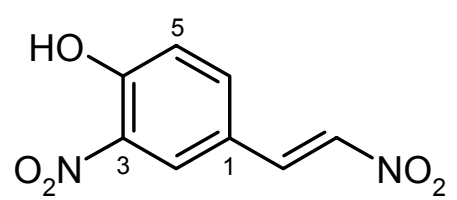
(d, $\left.{ }^{3} J=13.5 \mathrm{~Hz}, 1 \mathrm{H}, C H \mathrm{NO}_{2}\right), 7.87\left(\mathrm{~d},{ }^{3} J=13.5 \mathrm{~Hz}, 1 \mathrm{H}, C H \mathrm{CHNO}_{2}\right), 7.87\left(\mathrm{dd},{ }^{4} J=2.4 \mathrm{~Hz}\right.$, $\left.{ }^{3} J=8.7 \mathrm{~Hz}, 1 \mathrm{H}, 6-\mathrm{H}\right), 7.10\left(\mathrm{~d},{ }^{3} J=8.7 \mathrm{~Hz}, 1 \mathrm{H}, 5-\mathrm{H}\right) .-{ }^{13} \mathbf{C}-\mathbf{N M R}\left(\mathrm{CD}_{3} \mathrm{OD}, 150.8 \mathrm{MHz}\right): \delta=$ $160.8\left(\mathrm{C}_{\mathrm{q}}, \mathrm{C}-4\right), 145.8\left(\mathrm{C}_{\mathrm{q}}, \mathrm{C}-3\right), 138.6(\mathrm{CH}, \mathrm{CHCHNO} 2), 137.6\left(\mathrm{CH}, \mathrm{CHCHNO}{ }_{2}\right), 136.3$ (CH, C-6), 129.3 (CH, C-2), $123.6(\mathrm{CH}, \mathrm{C}-5), 121.8\left(\mathrm{C}_{\mathrm{q}}, \mathrm{C}-1\right)$. - EI-MS (70 eV): $m / z(\%)=$ $210\left(\left([\mathrm{M}]^{++}, 24\right), 163\right.$ (100), 118 (38), 89 (70), 63 (58). - UV (MeOH): $\lambda_{\max }(\lg \varepsilon)=222$ (4,20), 269 (4.04), 325 (4.20), 435 (sh) (3.54) nm. - IR (KBr): $\widetilde{v}\left(\mathrm{~cm}^{-1}\right)=3436,1640,1624$, $1537,1513,1504,1489,1342,1275,1174$.

\section{3,5-Dinitro-4-hydroxy-phenyl-essigsäuremethylester}

(48): $\mathrm{C}_{9} \mathrm{H}_{8} \mathrm{~N}_{2} \mathrm{O}_{7}$ (256), gelber Feststoff, UV-löschend (254 $\mathrm{nm}) .-\boldsymbol{R}_{\boldsymbol{f}}=0.36\left(\mathrm{CH}_{2} \mathrm{Cl}_{2} / \mathrm{MeOH} 9: 1\right) .-\boldsymbol{R}_{\boldsymbol{t}}=12.8 \mathrm{~min}(\mathrm{LC}-$ MS). $-{ }^{1} \mathbf{H}-\mathbf{N M R}\left(\mathrm{CD}_{3} \mathrm{OD}, 600 \mathrm{MHz}\right): \delta=7.96$ (s, 2H, 2-H,<smiles>COC(=O)Cc1cc([N+](=O)[O-])c(O)c([N+](=O)[O-])c1</smiles>
6-H), $3.70\left(\mathrm{~s}, 3 \mathrm{H}, \mathrm{COOCH}_{3}\right), 3.60$ (s, $\left.2 \mathrm{H}, \mathrm{CH}_{2} \mathrm{COO}\right) .-{ }^{13} \mathrm{C}-$ NMR (CD $\left.{ }_{3} \mathrm{OD}, 150.8 \mathrm{MHz}\right): \delta=173.7\left(\mathrm{C}_{\mathrm{q}}, \mathrm{COO}\right), 158.3\left(\mathrm{C}_{\mathrm{q}}, \mathrm{C}-4\right), 143.1\left(\mathrm{C}_{\mathrm{q}}, \mathrm{C}-3 . \mathrm{C}-5\right)$, 132.5 (CH, C-1), 114.4 (CH, C-2, C-6), $52.6\left(\mathrm{CH}_{2}, \mathrm{CH}_{2} \mathrm{COO}\right), 39.6\left(\mathrm{COOCH}_{3}\right)$. - EI-MS (70 $\mathrm{eV}): m / z(\%)=256\left(\left([\mathrm{M}]^{\circ+}, 14\right), 226(13), 197\right.$ (12), 59 (16), 44 (100). - HRMS ber. für $\mathrm{C}_{9} \mathrm{H}_{8} \mathrm{~N}_{2} \mathrm{O}_{7}$ : 256.03315, gef.: 256.03330. - UV (MeOH): $\lambda_{\max }(\lg \varepsilon)=228$ (4.33), 432 (3.85) nm. - IR (KBr): $\widetilde{v}\left(\mathrm{~cm}^{-1}\right)=3437,2925,1738,1631,1553,1439,1406,1384,1338,1265$, 1238, 1198, 1177, 1116. 


\section{3,5-Dinitro-4-hydroxy-phenyl-propionsäure (49):}

$\mathrm{C}_{9} \mathrm{H}_{8} \mathrm{~N}_{2} \mathrm{O}_{7}$ (256), gelber Feststoff, UV-löschend (254 nm).

$-\boldsymbol{R}_{\boldsymbol{f}}=0.16\left(\mathrm{CH}_{2} \mathrm{Cl}_{2} / \mathrm{MeOH} 9: 1\right) .-\boldsymbol{R}_{\boldsymbol{t}}=11.9 \mathrm{~min}(\mathrm{LC}-$

MS). - ${ }^{1} \mathbf{H}-\mathbf{N M R}\left(\mathrm{CD}_{3} \mathrm{OD}, 600 \mathrm{MHz}\right): \delta=8.04$ (s, 2H, 2$\mathrm{H}, 6-\mathrm{H}), 2.89\left(\mathrm{t},{ }^{3} \mathrm{~J}=7.0 \mathrm{~Hz}, 2 \mathrm{H}, \mathrm{CH}_{2} \mathrm{CH}_{2} \mathrm{COO}\right), 2.62\left(\mathrm{t},{ }^{3} \mathrm{~J}\right.$ $\left.=7.0 \mathrm{~Hz}, 2 \mathrm{H}, \mathrm{CH}_{2} \mathrm{CH}_{2} \mathrm{COO}\right) .{ }^{{ }^{13}} \mathbf{C}-\mathbf{N M R}\left(\mathrm{CD}_{3} \mathrm{OD}, 150.8\right.$<smiles>O=C(O)CCc1cc([N+](=O)[O-])c(O)c([N+](=O)[O-])c1</smiles>

$\mathrm{MHz}): \delta=176.2\left(\mathrm{C}_{\mathrm{q}}, \mathrm{COOH}\right), 152.7\left(\mathrm{C}_{\mathrm{q}}, \mathrm{C}-4\right), 141.7\left(\mathrm{C}_{\mathrm{q}}, \mathrm{C}-3, \mathrm{C}-5\right), 131.4(\mathrm{CH}, \mathrm{C}-2, \mathrm{C}-6)$, $128.4\left(\mathrm{C}_{\mathrm{q}}, \mathrm{C}-1\right), 36.1\left(\mathrm{CH}_{2}, \mathrm{CH}_{2} \mathrm{CH}_{2} \mathrm{COO}\right), 30.3\left(\mathrm{CH}_{2}, \mathrm{CH}_{2} \mathrm{CH}_{2} \mathrm{COO}\right)$. - (-)-ESI-MS: $m / z$ (\%) $=533[2 \mathrm{M}-2 \mathrm{H}+\mathrm{Na}]^{-}$(29), $255[\mathrm{M}-\mathrm{H}]^{-}(100)$. - HRMS ber. für $\mathrm{C}_{9} \mathrm{H}_{8} \mathrm{~N}_{2} \mathrm{O}_{7}$ : 256.03315, gef.: 256.03320. - UV (MeOH): $\lambda_{\max }(\lg \varepsilon)=226$ (3.92), 356 (3.16), 436 (3.39) nm. - IR (KBr): $\widetilde{v}\left(\mathrm{~cm}^{-1}\right)=3412,1712,1638,1542,1428,1343,1262$.

\section{3,5-Dinitro-4-hydroxy-phenyl-ethylchlorid (50):}

$\mathrm{C}_{8} \mathrm{H}_{7} \mathrm{~N}_{2} \mathrm{O}_{5} \mathrm{Cl}$ (246), gelber Feststoff, UV-löschend (254 nm). $\boldsymbol{R}_{\boldsymbol{f}}=0.44\left(\mathrm{CH}_{2} \mathrm{Cl}_{2} / \mathrm{MeOH} 9: 1\right) .-\boldsymbol{R}_{\boldsymbol{t}}=15.6 \mathrm{~min}(\mathrm{LC}-\mathrm{MS})$. ${ }^{1}$ H-NMR $\left(\mathrm{CD}_{3} \mathrm{OD}, 600 \mathrm{MHz}\right): \delta=8.02(\mathrm{~s}, 2 \mathrm{H}, 2-\mathrm{H}, 6-\mathrm{H}), 3.75$ $\left(\mathrm{t},{ }^{3} \mathrm{~J}=7.2 \mathrm{~Hz}, 2 \mathrm{H}, \mathrm{CH}_{2} \mathrm{CH}_{2} \mathrm{Cl}\right), 3.02\left(\mathrm{t},{ }^{3} \mathrm{~J}=7.2 \mathrm{~Hz}, 2 \mathrm{H}\right.$,<smiles>O=[N+]([O-])c1cc(CCCl)cc([N+](=O)[O-])c1O</smiles>
$\left.\mathrm{CH}_{2} \mathrm{CH}_{2} \mathrm{Cl}\right) .-{ }^{13} \mathbf{C}-\mathrm{NMR}\left(\mathrm{CD}_{3} \mathrm{OD}, 150.8 \mathrm{MHz}\right): \delta=159.6\left(\mathrm{C}_{\mathrm{q}}, \mathrm{C}-4\right), 144.2\left(\mathrm{C}_{\mathrm{q}}, \mathrm{C}-3, \mathrm{C}-5\right)$, $132.2(\mathrm{CH}, \mathrm{C}-2, \mathrm{C}-6), 119.1\left(\mathrm{C}_{\mathrm{q}}, \mathrm{C}-1\right), 46.0\left(\mathrm{CH}_{2}, \mathrm{CH}_{2} \mathrm{CH}_{2} \mathrm{Cl}\right), 38.2\left(\mathrm{CH}_{2}, \mathrm{CH}_{2} \mathrm{CH}_{2} \mathrm{Cl}\right)$. - EIMS $(70 \mathrm{eV}): m / z(\%)=246\left(\left([\mathrm{M}]^{\circ+}, 16\right), 197(100), 151\right.$ (16), 91 (98). - HRMS ber. für $\mathrm{C}_{8} \mathrm{H}_{7} \mathrm{~N}_{2} \mathrm{O}_{5} \mathrm{Cl}: 246.00435$, gef.: 246.00430. - UV (MeOH): $\lambda_{\max }(\lg \varepsilon)=230$ (4.16), 435 (3.68) nm. - IR (KBr): $\widetilde{v}\left(\mathrm{~cm}^{-1}\right)=3424,2926,1719,1638,1543,1429,1341,1256$.

\section{3,5-Dinitro-4-hydroxy-phenyl-2-chlor-propionsäure-}

methylester (51): $\mathrm{C}_{10} \mathrm{H}_{9} \mathrm{ClN}_{2} \mathrm{O}_{7}$ (304), orange Feststoff, UV-löschend (254 nm). $-\boldsymbol{R}_{\boldsymbol{f}}=0.41\left(\mathrm{CH}_{2} \mathrm{Cl}_{2} / \mathrm{MeOH} 9: 1\right)$. $-\boldsymbol{R}_{\boldsymbol{t}}=17.0 \mathrm{~min}(\mathrm{LC}-\mathrm{MS}) .-{ }^{\mathbf{1}} \mathbf{H}-\mathrm{NMR}\left(\mathrm{CD}_{3} \mathrm{OD}, 600\right.$ $\mathrm{MHz}): \delta=7.82\left(\mathrm{~s}, 2 \mathrm{H}, 2^{\prime}-\mathrm{H}, 6^{\prime}-\mathrm{H}\right), 4.56\left(\mathrm{t}, 1 \mathrm{H},{ }^{3} \mathrm{~J}=8.9\right.$<smiles>COC(=O)C(Cl)Cc1cc([N+](=O)[O-])c(O)c([N+](=O)[O-])c1</smiles>
$\mathrm{Hz}, 2-\mathrm{H}), 3.73\left(\mathrm{~s}, 3 \mathrm{H}, 1-\mathrm{OCH}_{3}\right), 3.18,3.06\left(\mathrm{ABX},{ }^{2} J=\right.$ $\left.15.1 \mathrm{~Hz},{ }^{3} J=8.9 \mathrm{~Hz}, 3-\mathrm{H}\right) .-(-)-\mathbf{A P C I}: m / z=303[\mathrm{M}-\mathrm{H}]$. $\mathbf{U V}(\mathrm{MeOH}): \lambda_{\max }(\lg \varepsilon)=213(\mathrm{~s}$, 3.58), 340 (2.89) nm. - IR (KBr): $\widetilde{v}\left(\mathrm{~cm}^{-1}\right)=3427,2925,2854,1740,1621,1545,1399$, $1258,1098,701$. 
N-(3,5-Dinitro-4-hydroxy-phenylethyl)acetamid (52): $\mathrm{C}_{10} \mathrm{H}_{11} \mathrm{~N}_{3} \mathrm{O}_{6}$ (269), gelber Feststoff, UV-löschend (254 $\mathrm{nm}) .-\boldsymbol{R}_{\boldsymbol{f}}=0.19\left(\mathrm{CH}_{2} \mathrm{Cl}_{2} / \mathrm{MeOH} 9: 1\right) .-\boldsymbol{R}_{\boldsymbol{t}}=10.3 \mathrm{~min}$ (LC-MS). $-{ }^{1} \mathbf{H}-\mathbf{N M R}\left(\left[\mathrm{D}_{6}\right] \mathrm{DMSO}, 300 \mathrm{MHz}\right): \delta=7.82$ (t br, 1H, NH), $7.64(\mathrm{~s}, 2 \mathrm{H}, 2-\mathrm{H}, 6-\mathrm{H}), 3.17(\mathrm{~m}, 2 \mathrm{H}$,<smiles>CC(=O)NCCc1cc([N+](=O)[O-])c(O)c([N+](=O)[O-])c1</smiles>
$\left.\mathrm{CH}_{2} \mathrm{CH}_{2} \mathrm{NH}\right), 2.48\left(\mathrm{~m}, 2 \mathrm{H}, \mathrm{CH}_{2} \mathrm{CH}_{2} \mathrm{NH}\right), 1.77(\mathrm{~s}, 3 \mathrm{H}$, Ac). $-{ }^{13} \mathbf{C}-\mathbf{N M R}\left(\left[\mathrm{D}_{6}\right] \mathrm{DMSO}, 150.8 \mathrm{MHz}\right): \delta=169.0\left(\mathrm{C}_{\mathrm{q}}, \mathrm{CH}_{3} \mathrm{CO}\right), 158.5\left(\mathrm{C}_{\mathrm{q}}, \mathrm{C}-4\right), 142.6$ $\left(\mathrm{C}_{\mathrm{q}}, \mathrm{C}-3, \mathrm{C}-5\right), 130.7(\mathrm{CH}, \mathrm{C}-2, \mathrm{C}-6), 114.4\left(\mathrm{C}_{\mathrm{q}}, \mathrm{C}-1\right), 40.0\left(\mathrm{CH}_{2}, \mathrm{CH}_{2} \mathrm{CH}_{2} \mathrm{NH}\right), 33.2\left(\mathrm{CH}_{2}\right.$, $\left.\mathrm{CH}_{2} \mathrm{CH}_{2} \mathrm{NH}\right), 22.5\left(\mathrm{CH}_{3}, \mathrm{CH}_{3} \mathrm{CO}\right)$. - (-)-ESI-MS: $m / z(\%)=537$ [2M-H] $]^{-}(45), 268[\mathrm{M}-\mathrm{H}]^{-}$ (100). - HRMS ber. für $\mathrm{C}_{10} \mathrm{H}_{11} \mathrm{~N}_{3} \mathrm{O}_{6}: 269.06478$, gef.: 269.06480. - UV (MeOH): $\lambda_{\max }(\lg \varepsilon)$ $=225(3.80), 443(3.21) \mathrm{nm} .-\mathbf{I R}(\mathrm{KBr}): \widetilde{v}\left(\mathrm{~cm}^{-1}\right)=3421,2928,1637,1543,1460,1384$, $1362,1338,1310,1253,1205$.

\section{2,6-Dinitro-4-(2'-nitroethenyl)phenol (53): $\quad \mathrm{C}_{8} \mathrm{H}_{5} \mathrm{~N}_{3} \mathrm{O}_{7}$}

(255), orange Feststoff, UV-löschend (254 nm). $-\boldsymbol{R}_{\boldsymbol{f}}=0.53$ $\left(\mathrm{CH}_{2} \mathrm{Cl}_{2} / \mathrm{MeOH}\right.$ 9:1). $-{ }^{1} \mathbf{H}-\mathbf{N M R}\left(\mathrm{CD}_{3} \mathrm{OD}, 600 \mathrm{MHz}\right): \delta=$ 8.24 (s, 2H, 3-H, 5-H), 7.99 (d, $\left.{ }^{3} J=13.5 \mathrm{~Hz}, 1 \mathrm{H}, 2^{\prime}-\mathrm{H}\right), 7.76$ $\left(\mathrm{d},{ }^{3} J=13.5 \mathrm{~Hz}, 1 \mathrm{H}, 1^{\prime}-\mathrm{H}\right)$. - (-)-ESI-MS: $m / z=531$ [2M-<smiles>O=[N+]([O-])C=Cc1cc([N+](=O)[O-])c(O)c([N+](=O)[O-])c1</smiles$2 \mathrm{H}+\mathrm{Na}]^{-}, 254[\mathrm{M}-\mathrm{H}]^{-}$.

Pyriculamid (55): $\mathrm{C}_{14} \mathrm{H}_{15} \mathrm{~N}_{3} \mathrm{O}_{5}$ (305), gelber Feststoff, UV-löschend $(254 \mathrm{~nm}) .-\boldsymbol{R}_{\boldsymbol{f}}=0.45\left(\mathrm{CH}_{2} \mathrm{Cl}_{2} / \mathrm{MeOH}\right.$ 9:1). $\quad-\boldsymbol{R}_{\boldsymbol{t}}=9.5 \mathrm{~min}$ (LC-MS). $\quad{ }^{1} \mathbf{H}-\mathbf{N M R}$ ([D $\left.\left.{ }_{6}\right] \mathrm{DMSO}, 300 \mathrm{MHz}\right): \delta=8.14\left(\mathrm{~d},{ }^{3} J=4.0 \mathrm{~Hz}, 1 \mathrm{H}\right.$, NH), $7.70\left(\mathrm{~d},{ }^{4} J=2.3 \mathrm{~Hz}, 1 \mathrm{H}, 5-\mathrm{H}\right), 7.34\left(\mathrm{dd},{ }^{3} J=8.4\right.$<smiles>O=C1NC(Cc2ccc(O)c([N+](=O)[O-])c2)C(=O)N2CCCC12</smiles>
$\left.\mathrm{Hz},{ }^{4} J=2.3 \mathrm{~Hz}, 1 \mathrm{H}, 9-\mathrm{H}\right), 7.08\left(\mathrm{~d},{ }^{3} J=8.4 \mathrm{~Hz}, 1 \mathrm{H}, 8-\right.$ H), $3.94(\mathrm{~m}, 1 \mathrm{H}, 2-\mathrm{H}), 3.48\left(\mathrm{~m}, 1 \mathrm{H}, 2^{\prime}-\mathrm{H}\right), 3.46\left(\mathrm{~m}, 1 \mathrm{H}, 3-\mathrm{H}_{\mathrm{A}}\right), 3.27\left(\mathrm{~m}, 1 \mathrm{H}, 3-\mathrm{H}_{\mathrm{B}}\right), 2.96(\mathrm{~m}$, 2H, 5'-H), $2.06\left(\mathrm{~m}, 1 \mathrm{H}, 3^{\prime}-\mathrm{H}_{\mathrm{A}}\right), 1.83\left(\mathrm{~m}, 1 \mathrm{H}, 4^{\prime}-\mathrm{H}_{\mathrm{A}}\right), 1.68$ (m, 2H, 3'- $\left.\mathrm{H}_{\mathrm{A}}, 4{ }^{\prime}-\mathrm{H}_{\mathrm{B}}\right) .{ }^{-13} \mathbf{C}-\mathbf{N M R}$ ([D $]$ DMSO, $150.8 \mathrm{MHz}): \delta=168.2\left(\mathrm{C}_{\mathrm{q}}, \mathrm{C}-1\right), 164.5\left(\mathrm{C}_{\mathrm{q}}, \mathrm{C}-1^{\prime}\right), 151.1\left(\mathrm{C}_{\mathrm{q}}, \mathrm{C}-7\right), 136.6(\mathrm{CH}$, C-5), $136.2\left(\mathrm{C}_{\mathrm{q}}, \mathrm{C}-6\right), 127.4$ (C $\left.\mathrm{q}, \mathrm{C}-4\right), 125.7$ (CH, C-9), 119.1 (CH, C-8), 57.8 (CH, C-2), $57.3\left(\mathrm{CH}, \mathrm{C}-2^{\prime}\right), 44.7\left(\mathrm{CH}_{2}, \mathrm{C}-3\right), 37.7\left(\mathrm{CH}_{2}, \mathrm{C}-5\right.$ ') $28.5\left(\mathrm{CH}_{2}, \mathrm{C}-3\right.$ '), $21.4\left(\mathrm{CH}_{2}, \mathrm{C}-4^{\prime}\right)$. - (+)ESI-MS: $m / z(\%)=655[2 \mathrm{M}-\mathrm{H}+2 \mathrm{Na}]^{+}(100), 633[2 \mathrm{M}+\mathrm{Na}]^{+}(25) .-(-)$-ESI-MS: $m / z(\%)=$ $631[2 \mathrm{M}-2 \mathrm{H}+\mathrm{Na}]^{-}(100), 304[\mathrm{M}-\mathrm{H}]^{-}(55) .-\mathbf{U V}(\mathrm{MeOH}): \lambda_{\max }(\lg \varepsilon)=208$ (3.40), 273 (2.42), 354 (2.08) nm. - IR (KBr): $\widetilde{v}\left(\mathrm{~cm}^{-1}\right)=3439,3191,3081,2930,2862,1623,1524,1472$, $1445,1356,1335,1280$. 


\section{7-Hydroxy-3-(4-hydroxy-2-nitrophenyl)-4H-1-}

\section{benzopyran-4-on (3'-Nitro-daidzein, 56):}

$\mathrm{C}_{15} \mathrm{H}_{9} \mathrm{NO}_{6}$ (299), gelber Feststoff, UV-löschend $(254 \mathrm{~nm}) .-\boldsymbol{R}_{\boldsymbol{f}}=0.59\left(\mathrm{CH}_{2} \mathrm{Cl}_{2} / \mathrm{MeOH} 9: 1\right) .-\boldsymbol{R}_{\boldsymbol{t}}=$ $16.0 \mathrm{~min}$ (LC-MS). - ${ }^{\mathbf{1}} \mathbf{H}-\mathbf{N M R}$ ([D $\left.\mathrm{D}_{6}\right] \mathrm{DMSO}, 300$<smiles>O=c1c(-c2ccc(O)c([N+](=O)[O-])c2)coc2cc(O)ccc12</smiles>
$\mathrm{MHz}): \delta=10.90$ (s br, 1H, OH), 8.45 (s, 1H, 2-H), $8.15\left(\mathrm{~d},{ }^{4} J=2.1 \mathrm{~Hz}, 1 \mathrm{H}, 2\right.$ '-H), $7.97\left(\mathrm{~d},{ }^{3} J=8.8 \mathrm{~Hz}, 1 \mathrm{H}, 5-\mathrm{H}\right), 7.71\left(\mathrm{dd},{ }^{3} J=8.8 \mathrm{~Hz},{ }^{4} J=2.1\right.$ $\left.\mathrm{Hz}, 1 \mathrm{H}, 6^{\prime}-\mathrm{H}\right), 7.12$ (d, $\left.{ }^{3} J=8.8 \mathrm{~Hz}, 1 \mathrm{H}, 5^{\prime}-\mathrm{H}\right), 6.94\left(\mathrm{dd},{ }^{3} J=8.8 \mathrm{~Hz},{ }^{4} J=2.1 \mathrm{~Hz}, 1 \mathrm{H}, 6-\mathrm{H}\right)$, $6.88\left(\mathrm{~d},{ }^{4} J=2.1 \mathrm{~Hz}, 1 \mathrm{H}, 8-\mathrm{H}\right) .-{ }^{13} \mathrm{C}-\mathbf{N M R}\left(\left[\mathrm{D}_{6}\right] \mathrm{DMSO}, 150.8 \mathrm{MHz}\right): \delta=174.3\left(\mathrm{C}_{\mathrm{q}}, \mathrm{C}-4\right)$, $162.8\left(\mathrm{C}_{\mathrm{q}}, \mathrm{C}-7\right), 157.4\left(\mathrm{C}_{\mathrm{q}}, \mathrm{C}-8 \mathrm{a}\right), 153.7(\mathrm{CH}, \mathrm{C}-2), 153.0\left(\mathrm{C}_{\mathrm{q}}, \mathrm{C}-4{ }^{\prime}\right), 136.5\left(\mathrm{C}_{\mathrm{q}}, \mathrm{C}-3\right.$ '), 135.3 (CH, C-6’), 127.2 (CH, C-5), 125.2 (CH, C-2'), 122.0 (C $\left.\mathrm{C}_{\mathrm{q}}, \mathrm{C}-1^{\prime}\right), 121.4\left(\mathrm{C}_{\mathrm{q}}, \mathrm{C}-3\right), 119.6(\mathrm{CH}$, C-5'), $116.4\left(\mathrm{C}_{\mathrm{q}}, \mathrm{C}-4 \mathrm{a}\right), 115.3(\mathrm{CH}, \mathrm{C}-6), 102.1(\mathrm{CH}, \mathrm{C}-8)$. - (+)-ESI-MS: $m / z(\%)=621$ $[2 \mathrm{M}+\mathrm{Na}]^{+}(78), 300[\mathrm{M}+\mathrm{H}]^{+}(18) .-(-)$-ESI-MS: $m / z(\%)=597\left[^{2} \mathrm{M}-\mathrm{H}\right]^{-}(40), 298[\mathrm{M}-\mathrm{H}]^{-}$ (100). - DCI-MS $\left(\mathrm{NH}_{3}\right): m / z(\%)=456\left[2 \mathrm{M}+\mathrm{NH}_{4}\right]^{+}(14), 237\left[\mathrm{M}+\mathrm{NH}_{4}\right]^{+}(100) .-\mathbf{U V}$ $(\mathrm{MeOH}): \lambda_{\max }(\lg \varepsilon)=243$ (3.99), 300 (sh, 3.67), 359 (3.22) nm. $-\mathbf{I R}(\mathrm{KBr}): \widetilde{v}\left(\mathrm{~cm}^{-1}\right)=3429$, $3281,1588,1578,1537,1426,1385,1310,1266,1240,1179$.

\section{5,7-Dihydroxy-3(3,5-dinitro-4-hydroxy-phenyl)-}

4H-1-benzopyran-4-on (3',5'-Dinitro-genistein,

57): $\mathrm{C}_{15} \mathrm{H}_{8} \mathrm{~N}_{2} \mathrm{O}_{9}$ (360), orange Feststoff, UVlöschend (254 nm). $-\boldsymbol{R}_{\boldsymbol{f}}=0.21\left(\mathrm{CH}_{2} \mathrm{Cl}_{2} / \mathrm{MeOH} 9: 1\right)$.

$-{ }^{1} \mathbf{H}-\mathbf{N M R}\left(\left[\mathrm{D}_{6}\right] \mathrm{DMSO}, 300 \mathrm{MHz}\right): \delta=12.78(\mathrm{br}$, $1 \mathrm{H}, \mathrm{OH}), 8.27$ (s, 1H, 2-H), 8.03 (s, 2H, 2'-H, 6'-H),<smiles>O=c1c(-c2cc([N+](=O)[O-])c(O)c([N+](=O)[O-])c2)coc2cc(O)cc(O)c12</smiles>
$6.12\left(\mathrm{~d},{ }^{4} J=2.1 \mathrm{~Hz}, 1 \mathrm{H}, 8-\mathrm{H}\right), 6.00\left(\mathrm{~d},{ }^{4} J=2.1 \mathrm{~Hz}, 1 \mathrm{H}, 6-\mathrm{H}\right) .-{ }^{13} \mathbf{C}-\mathbf{N M R}\left(\left[\mathrm{D}_{6}\right] \mathrm{DMSO}, 150.8\right.$ $\mathrm{MHz}): \delta=178.9\left(\mathrm{C}_{\mathrm{q}}, \mathrm{C}-4\right), 161.6\left(\mathrm{C}_{\mathrm{q}}, \mathrm{C}-5\right), 159.0\left(\mathrm{C}_{\mathrm{q}}, \mathrm{C}-4^{\prime}\right), 157.9\left(\mathrm{C}_{\mathrm{q}}, \mathrm{C}-8 \mathrm{a}\right), 152.6(\mathrm{CH}, \mathrm{C}-$ 2), $142.9\left(\mathrm{C}_{\mathrm{q}}, \mathrm{C}-3^{\prime}\right), 142.9\left(\mathrm{C}_{\mathrm{q}}, \mathrm{C}-5^{\prime}\right), 130.4\left(\mathrm{CH}, \mathrm{C}-2^{\prime}\right), 130.4\left(\mathrm{CH}, \mathrm{C}-6^{\prime}\right), 119.9\left(\mathrm{C}_{\mathrm{q}}, \mathrm{C}^{\prime} 1^{\prime}\right)$, 105.7 (C $\left.\mathrm{C}_{\mathrm{q}}, \mathrm{C}-3\right), 102.3\left(\mathrm{C}_{\mathrm{q}}, \mathrm{C}-4 \mathrm{a}\right), 100.2$ (CH, C-6), 94.3 (CH, C-8). - (-)-APCI-MS: $m / z$ (\%) $=359$ [M-H] $^{-}$(100). - HRMS ber. für $\mathrm{C}_{15} \mathrm{H}_{8} \mathrm{~N}_{2} \mathrm{O}_{9}$ : 360.02297, gef:: 360.02307. - UV $(\mathrm{MeOH}): \lambda_{\max }(\lg \varepsilon)=268(4.12), 434(3.29) \mathrm{nm}$.

2-Nitro- $\boldsymbol{N}_{\mathbf{B}}$-acetyltryptamin (58): $\mathrm{C}_{12} \mathrm{H}_{13} \mathrm{~N}_{3} \mathrm{O}_{3} \quad$ (247), gelber Feststoff, UV-löschend $(254 \mathrm{~nm}) .-\boldsymbol{R}_{\boldsymbol{f}}=0.55$ $\left(\mathrm{CH}_{2} \mathrm{Cl}_{2} / \mathrm{MeOH}\right.$ 9:1). $-\boldsymbol{R}_{\boldsymbol{t}}=12.8 \mathrm{~min}(\mathrm{LC}-\mathrm{MS}) .-{ }^{\mathbf{1}} \mathbf{H}-$ NMR $\left(\mathrm{CD}_{3} \mathrm{OD}, 300 \mathrm{MHz}\right): \delta=7.74(\mathrm{~d}$ br, $1 \mathrm{H}, 4-\mathrm{H})$,<smiles>CC(=O)NCCc1c([N+](=O)[O-])[nH]c2ccccc12</smiles> 
$7.41(\mathrm{~m}, 2 \mathrm{H}, 6-\mathrm{H}, 7-\mathrm{H}), 7.18(\mathrm{~m}, 1 \mathrm{H}, 5-\mathrm{H}), 3.51\left(\mathrm{t},{ }^{3} \mathrm{~J}=6.8 \mathrm{~Hz}, 2 \mathrm{H}, 9-\mathrm{H}\right), 3.37\left(\mathrm{t},{ }^{3} J=6.8 \mathrm{~Hz}\right.$, 2H, 8-H), 1.78 (s, 3H, Ac). $-{ }^{13}$ C-NMR (CD $\left.{ }_{3} \mathrm{OD}, 150.8 \mathrm{MHz}\right): \delta=173.4\left(\mathrm{C}_{\mathrm{q}}, \mathrm{C}-11\right), 139.2$ $\left(\mathrm{C}_{\mathrm{q}}, \mathrm{C}-2\right), 135.9\left(\mathrm{C}_{\mathrm{q}}, \mathrm{C}-7 \mathrm{a}\right), 129.0(\mathrm{CH}, \mathrm{C}-6), 128.2\left(\mathrm{C}_{\mathrm{q}}, \mathrm{C}-3 \mathrm{a}\right), 122.7(\mathrm{CH}, \mathrm{C}-4), 122.4(\mathrm{CH}$, C-5), $117.9\left(\mathrm{C}_{\mathrm{q}}, \mathrm{C}-3\right), 113.7$ (CH, C-7), $40.1\left(\mathrm{CH}_{2}, \mathrm{C}-9\right), 25.5\left(\mathrm{CH}_{2}, \mathrm{C}-8\right), 22.5\left(\mathrm{CH}_{3}, \mathrm{C}-12\right)$. $(+)$-ESI-MS: $m / z(\%)=517[2 \mathrm{M}+\mathrm{Na}]^{+}(72), 270[\mathrm{M}+\mathrm{Na}]^{+}(100) .-(-)-$ ESI-MS: $m / z(\%)=$ 515 [2M-2H+Na] $]^{-}$(19), 246 [M-H] $]^{-}$(100). - HRMS ber. für $\mathrm{C}_{12} \mathrm{H}_{13} \mathrm{~N}_{3} \mathrm{O}_{3}$ : 247.09569, gef.: 247.09567. - UV (MeOH): $\lambda_{\max }(\lg \varepsilon)=216$ (4.02), 244 (3.84), 355 (4.00) nm. - IR (KBr): $\widetilde{v}\left(\mathrm{~cm}^{-1}\right)=2924,2854,1656,1547,1452,1386,1290$.

\section{6-Nitro- $\boldsymbol{N}_{\boldsymbol{\beta}}$-acetyltryptamin (59): $\quad \mathrm{C}_{12} \mathrm{H}_{13} \mathrm{~N}_{3} \mathrm{O}_{3}$} (247), gelber Feststoff, UV-löschend (254 nm). $\boldsymbol{R}_{\boldsymbol{f}}=0.55\left(\mathrm{CH}_{2} \mathrm{Cl}_{2} / \mathrm{MeOH} 9: 1\right) .-\boldsymbol{R}_{\boldsymbol{t}}=12.9 \mathrm{~min}$ (LC-MS). - ${ }^{\mathbf{1}} \mathbf{H}-\mathbf{N M R}\left(\mathrm{CD}_{3} \mathrm{OD}, 300 \mathrm{MHz}\right): \delta=$<smiles>CC(=O)NCCc1c[nH]c2cc([N+](=O)[O-])ccc12</smiles>
$8.31\left(\mathrm{~d},{ }^{4} J=1.8 \mathrm{~Hz}, 1 \mathrm{H}, 7-\mathrm{H}\right), 7.92\left(\mathrm{dd},{ }^{3} J=8.7\right.$ $\left.\mathrm{Hz},{ }^{4} J=1.8 \mathrm{~Hz}, 1 \mathrm{H}, 5-\mathrm{H}\right), 7.68\left(\mathrm{~d},{ }^{3} J=8.7 \mathrm{~Hz}, 1 \mathrm{H}, 4-\mathrm{H}\right), 7.46(\mathrm{~s}, 1 \mathrm{H}, 2-\mathrm{H}), 3.46\left(\mathrm{t},{ }^{3} J=6.8\right.$ Hz, 2H, 9-H), $2.97\left(\mathrm{t},{ }^{3} J=6.8 \mathrm{~Hz}, 2 \mathrm{H}, 8-\mathrm{H}\right), 1.90$ (s, 3H, Ac). - (+)-ESI-MS: $m / z(\%)=517$ $[2 \mathrm{M}+\mathrm{Na}]^{+}(38), 270[\mathrm{M}+\mathrm{Na}]^{+}(100) .-(-)$-ESI-MS: $m / z(\%)=246[\mathrm{M}-\mathrm{H}]^{-}(100)$.

7-Nitro- $\boldsymbol{N}_{\boldsymbol{\beta}}$-acetyltryptamin (60): $\mathrm{C}_{12} \mathrm{H}_{13} \mathrm{~N}_{3} \mathrm{O}_{3} \quad$ (247), gelber Feststoff, UV-löschend $(254 \mathrm{~nm}) .-\boldsymbol{R}_{\boldsymbol{f}}=0.55$ $\left(\mathrm{CH}_{2} \mathrm{Cl}_{2} / \mathrm{MeOH}\right.$ 9:1). $-\boldsymbol{R}_{\boldsymbol{t}}=12.9 \min (\mathrm{LC}-\mathrm{MS}) .-{ }^{\mathbf{1}} \mathbf{H}-$ NMR $\left(\mathrm{CD}_{3} \mathrm{OD}, 300 \mathrm{MHz}\right): \delta=7.78\left(\mathrm{dd},{ }^{3} J=7.6 \mathrm{~Hz},{ }^{4} J\right.$ $=1.8 \mathrm{~Hz}, 1 \mathrm{H}, 6-\mathrm{H}), 7.71\left(\mathrm{dd},{ }^{3} J=8.4 \mathrm{~Hz},{ }^{4} J=1.8 \mathrm{~Hz}\right.$,<smiles>CC(=O)NCCc1c[nH]c2c([N+](=O)[O-])cccc12</smiles>
1H, 7-H, 4-H), 7.38 (t, $\left.{ }^{3} J=8.2 \mathrm{~Hz}, 1 \mathrm{H}, 5-\mathrm{H}\right), 7.20$ (s, 1H, 2-H), 3.34 (t, ${ }^{3} J=6.8 \mathrm{~Hz}, 2 \mathrm{H}, 9-$ H), $3.04\left(\mathrm{t},{ }^{3} J=6.8 \mathrm{~Hz}, 2 \mathrm{H}, 8-\mathrm{H}\right), 1.89(\mathrm{~s}, 3 \mathrm{H}, \mathrm{Ac}) .-(+)$-ESI-MS: $m / z(\%)=517[2 \mathrm{M}+\mathrm{Na}]^{+}$ (38), $270[\mathrm{M}+\mathrm{Na}]^{+}(100)$. - (-)-ESI-MS: $m / z(\%)=246[\mathrm{M}-\mathrm{H}]^{-}(100)$.

\section{1-Acetyl-1,2,3,3a,8,8a-hexahydropyrrolo[2,3-b]-3a-nitro-}

indol (Salegentipyrrol A, 61): $\mathrm{C}_{12} \mathrm{H}_{13} \mathrm{~N}_{3} \mathrm{O}_{3}$ (247), farbloses Öl, UV-löschend (254 nm). $-\boldsymbol{R}_{\boldsymbol{f}}=0.60\left(\mathrm{CH}_{2} \mathrm{Cl}_{2} / \mathrm{MeOH} 9: 1\right)$. Anfärbeverhalten: Anisaldehyd (orange), Ehrlich (grün). $-\boldsymbol{R}_{\boldsymbol{t}}=$

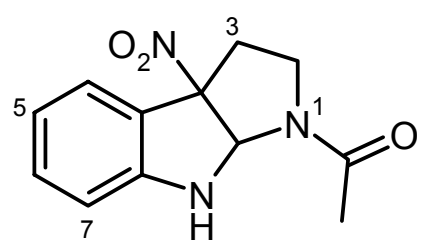
$12.8 \min (\mathrm{LC}-\mathrm{MS}) .-{ }^{\mathbf{1}} \mathbf{H}-\mathbf{N M R}\left(\mathrm{CDCl}_{3}, 300 \mathrm{MHz}\right): \delta=7.42$ $\left(\mathrm{dd},{ }^{3} J=7.7 \mathrm{~Hz},{ }^{4} J=2.1 \mathrm{~Hz}, 1 \mathrm{H}, 4-\mathrm{H}\right), 7.24\left(\mathrm{dt},{ }^{3} J=7.7 \mathrm{~Hz},{ }^{4} J=2.0 \mathrm{~Hz}, 1 \mathrm{H}, 6-\mathrm{H}\right), 6.84$ (dt, $\left.{ }^{3} J=7.7 \mathrm{~Hz},{ }^{4} J=2.1 \mathrm{~Hz}, 1 \mathrm{H}, 5-\mathrm{H}\right), 6.66\left(\mathrm{dd},{ }^{3} J=7.7 \mathrm{~Hz},{ }^{4} J=2.0 \mathrm{~Hz}, 1 \mathrm{H}, 7-\mathrm{H}\right), 6.23(\mathrm{~s}, 1 \mathrm{H}$, 8a-H), 5.34 (br, 1H, 8-NH), $3.84\left(\mathrm{ddd},{ }^{2} J=10.5 \mathrm{~Hz},{ }^{3} J=8.3 \mathrm{~Hz},{ }^{3} J=2.0 \mathrm{~Hz}, 1 \mathrm{H}, 2-\mathrm{H}_{\mathrm{A}}\right), 3.40$ 
(ddd, $\left.{ }^{2} J=10.5 \mathrm{~Hz},{ }^{3} J=10.5 \mathrm{~Hz},{ }^{3} J=6.3 \mathrm{~Hz}, 1 \mathrm{H}, 2-\mathrm{H}_{\mathrm{B}}\right), 3.01\left(\mathrm{ddd},{ }^{2} J=12.9 \mathrm{~Hz},{ }^{3} J=10.5 \mathrm{~Hz}\right.$, $\left.{ }^{3} J=8.3 \mathrm{~Hz}, 1 \mathrm{H}, 3-\mathrm{H}_{\mathrm{A}}\right), 2.81\left(\mathrm{ddd},{ }^{2} J=12.9 \mathrm{~Hz},{ }^{3} J=6.3 \mathrm{~Hz},{ }^{3} J=2.0 \mathrm{~Hz}, 1 \mathrm{H}, 3-\mathrm{H}_{\mathrm{B}}\right.$ ), 2.06 (s, $\left.3 \mathrm{H}, \mathrm{COCH}_{3}\right) .-{ }^{13} \mathbf{C}-\mathbf{N M R}\left(\mathrm{CDCl}_{3}, 150.8 \mathrm{MHz}\right): \delta=170.1\left(\mathrm{C}_{\mathrm{q}}, \mathrm{CO}\right), 150.3\left(\mathrm{C}_{\mathrm{q}}, \mathrm{C}-7 \mathrm{a}\right), 132.4$ (CH, C-6), 125.0 (CH, C-4), 121.8 (C $\left.\mathrm{C}_{\mathrm{q}}, \mathrm{C}-3 \mathrm{~b}\right), 119.6(\mathrm{CH}, \mathrm{C}-5), 110.5(\mathrm{CH}, \mathrm{C}-7), 99.2\left(\mathrm{C}_{\mathrm{q}}, \mathrm{C}-\right.$ 3a), 78.5 (CH, C-8a), $46.8\left(\mathrm{CH}_{2}, \mathrm{C}-2\right), 36.1\left(\mathrm{CH}_{2}, \mathrm{C}-3\right), 22.2\left(\mathrm{CH}_{3}, \mathrm{COCH}_{3}\right)$. - DCI-MS $\left(\mathrm{NH}_{3}\right): m / z(\%)=512\left[2 \mathrm{M}+\mathrm{NH}_{4}\right]^{+}(5), 495[2 \mathrm{M}+\mathrm{H}]^{+}(19), 265\left[\mathrm{M}+\mathrm{NH}_{4}\right]^{+}(46), 248[\mathrm{M}+\mathrm{H}]^{+}$ (100). - HRMS ber. für $\mathrm{C}_{12} \mathrm{H}_{13} \mathrm{~N}_{3} \mathrm{O}_{3}: 247.09569$, gef.: 247.09581. - UV (MeOH): $\lambda_{\max }(\lg \varepsilon)$ $=239$ (3.74), 302 (3.04) nm. - IR $(\mathrm{KBr}): \widetilde{v}\left(\mathrm{~cm}^{-1}\right)=2927,2856,1636,1548,1438,1385$.

\section{1-Acetyl-1,2,3,3a,8,8a-hexahydropyrrolo[2,3-b]-3a,7-dini-}

tro-indol (Salegentipyrrol B, 64): $\mathrm{C}_{12} \mathrm{H}_{12} \mathrm{~N}_{4} \mathrm{O}_{5}$ (292), gelbes Ö1, UV-löschend (254 nm). $-\boldsymbol{R}_{\boldsymbol{f}}=0.49\left(\mathrm{CH}_{2} \mathrm{Cl}_{2} / \mathrm{MeOH} 9: 1\right)$. ${ }^{1} \mathbf{H}-\mathbf{N M R}\left(\mathrm{CDCl}_{3}, 600 \mathrm{MHz}\right): \delta=8.10\left(\mathrm{~d},{ }^{3} \mathrm{~J}=7.8 \mathrm{~Hz}, 1 \mathrm{H}, 4-\right.$ H), $7.70\left(\mathrm{~d},{ }^{3} J=7.8 \mathrm{~Hz}, 1 \mathrm{H}, 6-\mathrm{H}\right), 7.61$ (s br, 1H, 8-NH), 6.84

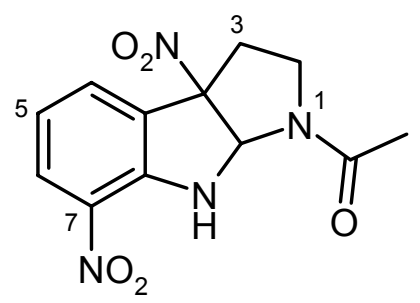
$\left(\mathrm{t},{ }^{3} J=7.8 \mathrm{~Hz}, 1 \mathrm{H}, 5-\mathrm{H}\right), 6.49(\mathrm{~s}, 1 \mathrm{H}, 8 \mathrm{a}-\mathrm{H}), 3.90\left(\mathrm{ddd},{ }^{2} J=\right.$ $\left.10.5 \mathrm{~Hz},{ }^{3} J=8.4 \mathrm{~Hz},{ }^{3} J=2.2 \mathrm{~Hz}, 1 \mathrm{H}, 2-\mathrm{H}_{\mathrm{A}}\right), 3.45\left(\mathrm{ddd},{ }^{2} J=10.5 \mathrm{~Hz},{ }^{3} J=10.5 \mathrm{~Hz},{ }^{3} J=6.1\right.$ $\left.\mathrm{Hz}, 1 \mathrm{H}, 2-\mathrm{H}_{\mathrm{B}}\right), 3.09\left(\mathrm{ddd},{ }^{2} J=13.2 \mathrm{~Hz},{ }^{3} J=10.5 \mathrm{~Hz},{ }^{3} J=8.4 \mathrm{~Hz}, 1 \mathrm{H}, 3-\mathrm{H}_{\mathrm{A}}\right), 2.82\left(\mathrm{ddd},{ }^{2} J=\right.$ $\left.13.2 \mathrm{~Hz},{ }^{3} \mathrm{~J}=6.1 \mathrm{~Hz},{ }^{3} \mathrm{~J}=2.2 \mathrm{~Hz}, 1 \mathrm{H}, 3-\mathrm{H}_{\mathrm{B}}\right), 2.10\left(\mathrm{~s}, 3 \mathrm{H}, 1-\mathrm{COCH}_{3}\right) .-(+)$-ESI-MS: $m / z(\%)$ $=607[2 \mathrm{M}+\mathrm{Na}]^{+}(27), 315[\mathrm{M}+\mathrm{Na}]^{+}(100) .-(-)$-ESI-MS: $m / z(\%)=291[\mathrm{M}-\mathrm{H}]^{-}(100)$. HRMS ber. für $\mathrm{C}_{12} \mathrm{H}_{12} \mathrm{~N}_{4} \mathrm{O}_{5}: 292.07968$, gef.: 292.08089 .

4,5-Dinitro-3-methoxy-phenol (65): $\mathrm{C}_{7} \mathrm{H}_{6} \mathrm{~N}_{2} \mathrm{O}_{6}$, orange Feststoff, UV-löschend (254 nm). $-\boldsymbol{R}_{\boldsymbol{f}}=0.51\left(\mathrm{CH}_{2} \mathrm{Cl}_{2} / \mathrm{MeOH} 9: 1\right) .-\boldsymbol{R}_{\boldsymbol{t}}=14.1$ $\min (\mathrm{LC}-\mathrm{MS}) .-{ }^{1} \mathbf{H}-\mathbf{N M R}\left(\mathrm{CD}_{3} \mathrm{OD}, 600 \mathrm{MHz}\right): \delta=8.59\left(\mathrm{~d},{ }^{4} J=2.4\right.$ $\mathrm{Hz}, 1 \mathrm{H}, 6-\mathrm{H}), 7.55$ (d, $\left.{ }^{4} \mathrm{~J}=2.4 \mathrm{~Hz}, 1 \mathrm{H}, 2-\mathrm{H}\right), 3.87$ (s, 3H, 3- $\left.\mathrm{OCH}_{3}\right)$. ${ }^{13}$ C-NMR $\left(\mathrm{CD}_{3} \mathrm{OD}, 150.8 \mathrm{MHz}\right): \delta=164.6\left(\mathrm{C}_{\mathrm{q}}, \mathrm{C}-1\right), 155.6\left(\mathrm{C}_{\mathrm{q}}, \mathrm{C}-\right.$<smiles>COc1cc(O)cc([N+](=O)[O-])c1[N+](=O)[O-]</smiles>
3), $135.2\left(\mathrm{C}_{\mathrm{q}}, \mathrm{C}-5\right), 132.2\left(\mathrm{C}_{\mathrm{q}}, \mathrm{C}-4\right), 118.3(\mathrm{CH}, \mathrm{C}-6), 106.7(\mathrm{CH}, \mathrm{C}-$ 2), $57.0\left(\mathrm{CH}_{3}, 3-\mathrm{OCH} 3\right)$. - (-)-APCI: $m / z=213[\mathrm{M}-\mathrm{H}]^{-}$. - HRMS ber. für $\mathrm{C}_{7} \mathrm{H}_{6} \mathrm{~N}_{2} \mathrm{O}_{6}$ : 214.02258, gef.: 214.02262. - UV (MeOH): $\lambda_{\max }(\lg \varepsilon)=213$ (4.22), 266 (3.92), 342 (3.68) nm. - IR (KBr): $\widetilde{v}\left(\mathrm{~cm}^{-1}\right)=3391,3109,2946,2835,1778,1607,1556,1449,1344,1261$, 1092, 1027, 946, 918, 804, 711. 
4,6-Dinitro-2-methoxy-phenol (66): $\mathrm{C}_{7} \mathrm{H}_{6} \mathrm{~N}_{2} \mathrm{O}_{6}$, orange Feststoff, UV-löschend (254 nm). $-\boldsymbol{R}_{\boldsymbol{f}}=0.50\left(\mathrm{CH}_{2} \mathrm{Cl}_{2} / \mathrm{MeOH} 9: 1\right) .-\boldsymbol{R}_{\boldsymbol{t}}=14.1$ $\min (\mathrm{LC}-\mathrm{MS}) .-{ }^{1} \mathbf{H}-\mathbf{N M R}\left(\mathrm{CD}_{3} \mathrm{OD}, 300 \mathrm{MHz}\right): \delta=8.51\left(\mathrm{~d},{ }^{4} \mathrm{~J}=2.7\right.$ $\mathrm{Hz}, 1 \mathrm{H}, 5-\mathrm{H}), 7.75$ (d, $\left.{ }^{4} \mathrm{~J}=2.7 \mathrm{~Hz}, 1 \mathrm{H}, 3-\mathrm{H}\right), 3.94$ (s, 3H, 2-OCH $)^{-}$${ }^{13}$ C-NMR $\left(\mathrm{CD}_{3} \mathrm{OD}, 150.8 \mathrm{MHz}\right): \delta=157.8\left(\mathrm{C}_{\mathrm{q}}, \mathrm{C}-1\right), 153.7\left(\mathrm{C}_{\mathrm{q}}, \mathrm{C}-\right.$ 2), $135.5\left(\mathrm{C}_{\mathrm{q}}, \mathrm{C}-6\right), 134.2\left(\mathrm{C}_{\mathrm{q}}, \mathrm{C}-4\right), 116.4(\mathrm{CH}, \mathrm{C}-5), 108.3(\mathrm{CH}, \mathrm{C}-$<smiles>COc1cc([N+](=O)[O-])cc([N+](=O)[O-])c1O</smiles>
3), $57.1\left(\mathrm{CH}_{3}, 2-\mathrm{OCH}_{3}\right)$. - EI-MS (70 eV): m/z (\%) = $214\left(\left([\mathrm{M}]^{\circ+}, 100\right), 197(70), 166(22)\right.$, 121 (28), 53 (29), 50 (33).

2,6-Dimethoxy-1,4-benzoquinon (67): $\mathrm{C}_{8} \mathrm{H}_{8} \mathrm{O}_{4}$ (168), gelber Feststoff. $-{ }^{1} \mathbf{H}-\mathbf{N M R}\left(\mathrm{CDCl}_{3}, 300 \mathrm{MHz}\right): \delta=5.84(\mathrm{~s}, 2 \mathrm{H}, 3-\mathrm{H}, 5-$ $\mathrm{H}), 3.80\left(\mathrm{~s}, 6 \mathrm{H}, 2 \mathrm{OCH}_{3}\right) .-{ }^{13} \mathbf{C}-\mathbf{N M R}\left(\mathrm{CDCl}_{3}, 150.8 \mathrm{MHz}\right): \delta=$ $186.8\left(\mathrm{C}_{\mathrm{q}}, \mathrm{C}-1\right), 176.7\left(\mathrm{C}_{\mathrm{q}}, \mathrm{C}-4\right), 157.3\left(\mathrm{C}_{\mathrm{q}}, \mathrm{C}-2, \mathrm{C}-6\right), 107.4(\mathrm{CH}$, C-3, C-5), $56.5\left(\mathrm{CH}_{3}, 2 \mathrm{OCH}_{3}\right)$. - EI-MS $(70 \mathrm{eV}): m / z(\%)=168$<smiles>COC1=CC(=O)C=C(OC)C1=O</smiles>
$\left(\left([\mathrm{M}]^{+}, 5\right), 104\right.$ (39), 91 (21), 80 (20), 69 (100), 53 (29).

3-Indolyl-ethyl-methylether $\quad \mathbf{( 6 8 ) :} \quad \mathrm{C}_{11} \mathrm{H}_{13} \mathrm{NO} \quad$ (175), farbloses Ö1, UV-löschend (254 nm). $-\boldsymbol{R}_{\boldsymbol{f}}=0.80$ $\left(\mathrm{CH}_{2} \mathrm{Cl}_{2} / \mathrm{MeOH}\right.$ 9:1). - Anfärbeverhalten: Anisaldehyd (rot), Ehrlich (violett). $-\boldsymbol{R}_{\boldsymbol{t}}=15.7 \mathrm{~min}(\mathrm{LC}-\mathrm{MS}) .-{ }^{\mathbf{1}} \mathbf{H}-$<smiles>COCCc1c[nH]c2ccccc12</smiles>
NMR $\left(\mathrm{CD}_{3} \mathrm{OD}, 600 \mathrm{MHz}\right): \delta=7.51\left(\mathrm{~d},{ }^{3} J=7.8 \mathrm{~Hz}, 1 \mathrm{H}, 7^{\prime}-\mathrm{H}\right), 7.31\left(\mathrm{~d},{ }^{3} J=7.8 \mathrm{~Hz}, 1 \mathrm{H}, 4^{\prime}-\right.$ H), 7.06 (t, ${ }^{3} J=7.8 \mathrm{~Hz}, 1 \mathrm{H}, 6$ '-H), 7.05 (s, 1H, 2'-H) 6.98 (t, $\left.{ }^{3} J=7.8 \mathrm{~Hz}, 1 \mathrm{H}, 5^{\prime}-\mathrm{H}\right), 3.66$ (t, $\left.{ }^{3} J=7.2 \mathrm{~Hz}, 2 \mathrm{H}, 1-\mathrm{H}\right), 3.37\left(\mathrm{~s}, 3 \mathrm{H}, \mathrm{OCH}_{3}\right), 2.99\left(\mathrm{t},{ }^{3} J=7.2 \mathrm{~Hz}, 2 \mathrm{H}, 2-\mathrm{H}\right) .-\mathbf{U V}(\mathrm{MeOH}): \lambda_{\max }$ $(\lg \varepsilon)=221,281 \mathrm{~nm} .-\mathbf{I R}(\mathrm{KBr}): \widetilde{v}\left(\mathrm{~cm}^{-1}\right)=3415,2926,1620,1457,1384,1339,1228$, 1107,742 .

Thymin (69): $\mathrm{C}_{5} \mathrm{H}_{6} \mathrm{~N}_{2} \mathrm{O}_{2}$ (126), weißer Feststoff, UV-löschend (254 $\mathrm{nm}) .-\boldsymbol{R}_{\boldsymbol{f}}=0.46\left(\mathrm{CH}_{2} \mathrm{Cl}_{2} / \mathrm{MeOH}\right.$ 9:1). - Keine Anfärbung. - ${ }^{1} \mathbf{H}-\mathbf{N M R}$ ([D $]$ DMSO, $300 \mathrm{MHz}): \delta=10.95(\mathrm{br}, 1 \mathrm{H}, 2-\mathrm{NH}), 10.55$ (br, 1H, 4$\mathrm{NH}), 7.23(\mathrm{~s}, 1 \mathrm{H}, 5-\mathrm{H}), 1.73\left(\mathrm{~s}, 3 \mathrm{H}, 6-\mathrm{CH}_{3}\right)$. - EI-MS $(70 \mathrm{eV}): m / z(\%)=$<smiles>Cc1c[nH]c(=O)[nH]c1=O</smiles>
$126\left(\left([\mathrm{M}]^{\circ+}, 100\right), 55(64)\right.$. 


\subsection{Actinomyces sp. T426}

Der aus litoralem Sediment in Feuerland isolierte mesophile Stamm T426 wuchs auf Agarplatten unter Ausbildung eines gelben Luft- sowie eines beigen Substratmycels.

\subsubsection{Primärscreening}

Beim biologischen Screening des Rohextraktes gegen verschiedenen Organismen (Tabelle 10) konnte lediglich eine cytotoxische Aktivität gegen die angeführten Zelllinien gefunden werden.

Tabelle 10 Für das biologische Screening verwendete Organismen bzw. Zelllinien.

\begin{tabular}{|c|c|c|}
\hline & Organismen & Konzentration ${ }^{*}$ \\
\hline Bakterien & B. brevis, B. subtilis, M. luteus, E. dissolvens & \multirow{4}{*}{$20 \mu \mathrm{l} /$ Plättchen } \\
\hline Pilze & $\begin{array}{l}\text { M. miehei, } N . \text { corylii, } P . \text { notatum, } P . \text { variotii, } \\
\text { C. parapsilosis, C. glabrata, C. krusei, } \\
\text { F. solani, } R . \text { oryzae }\end{array}$ & \\
\hline Pathogenes Hefe & C. albicans & \\
\hline Pathogene Bakterien & $\begin{array}{l}S . \text { aureus, } P \text {. vulgaris, } P \text {. fluorescens, } \\
P . \text { aeruginosa }\end{array}$ & \\
\hline Nematoden & C. elegans & $10 \mu \mathrm{l} / \mathrm{ml}$ \\
\hline Cytotoxizität & $\begin{array}{l}\text { MCF-7, HepG2, HeLA S3, Jurkat, Colo 320, } \\
\text { L1210, MDAMB231 }\end{array}$ & $60 \mu \mathrm{l} / 400 \mu \mathrm{l}$ \\
\hline
\end{tabular}

*Das Extrakt aus $200 \mathrm{ml}$ Kultur wurde in $3 \mathrm{ml} \mathrm{MeOH}$ aufgenommen.

\subsubsection{Kultivierung und Aufarbeitung}

Kultivierung und Aufarbeitung wurden am IBWF (Kaiserslautern) durchgeführt. Für die Vorkulturen wurde Stamm T426 in 500 ml Erlenmeyerkolben mit einer Schikane, welche mit $200 \mathrm{ml}$ B-Medium befüllt waren, bei $120 \mathrm{Upm}$ und $22{ }^{\circ} \mathrm{C}$ inkubiert. Die Fermentation der Hauptkulturen erfolgte ebenfalls bei $22{ }^{\circ} \mathrm{C}$ in B-Medium bei pH 8 . Die so erhaltene Kulturbrühe wurde mit Celite versetzt und filtriert. Das Mycel wurde daraufhin mit Essigester extra- 
hiert. Der pH-Wert des Kulturfiltrats wurde auf 4 eingestellt und anschließend ebenfalls mit Essigester extrahiert.

\subsubsection{Isolierung und Charakterisierung der Metaboliten}

Eine Vortrennung des Rohextraktes erfolgte bereits am IBWF (Kaiserslautern). Es wurden sechs Fraktionen (1 - 6) mittels Säulenchromatographie an Kieselgel unter Verwendung eines Stufengradienten aus Cyclohexan/Essigester (CH/EE 9:1, CH/EE 8:2, CH/EE 7:3, CH/EE 6:4, $\mathrm{CH} / \mathrm{EE}$ 5:5) erhalten. Nachfolgende Trennung an Sephadex LH-20 (MeOH) führte zu den Fraktionen $7-10$, welche jedoch nur in sehr geringen Mengen anfielen und folglich nur einem HPLC-ESI-MS/MS-Screening unterzogen werden konnten. Aus den Fraktionen 3-6 konnten durch Chromatographie an Sephadex LH-20 (MeOH) und mittels präparativer Dickschichtchromatographie $7.3 \mathrm{mg}$ Tryptophol (76) und $2.1 \mathrm{mg} p$-Hydroxybenzoesäuremethylester (77) isoliert werden.

Tryptophol (76): $\mathrm{C}_{10} \mathrm{H}_{11} \mathrm{NO}$ (161), farbloses Ö1, UVlöschend (254 nm). $-\boldsymbol{R}_{\boldsymbol{f}}=0.28\left(\mathrm{CH}_{2} \mathrm{Cl}_{2} / \mathrm{MeOH} 95: 5\right)$. - Anfärbeverhalten: Anisaldehyd (rot), Ehrlich (violett). $-\boldsymbol{R}_{\boldsymbol{t}}=$ $11.8 \mathrm{~min}$ (LC-MS). $-{ }^{\mathbf{1}} \mathbf{H}-\mathbf{N M R}\left(\mathrm{CDCl}_{3}, 300 \mathrm{MHz}\right): \delta=8.03$<smiles>OCCc1c[nH]c2ccccc12</smiles>
(s br, 1H, 1-NH), $7.62\left(\mathrm{~d},{ }^{3} J=8.2 \mathrm{~Hz}, 1 \mathrm{H}, 4-\mathrm{H}\right), 7.37\left(\mathrm{~d},{ }^{3} J=8.2 \mathrm{~Hz}, 1 \mathrm{H}, 7-\mathrm{H}\right), 7.20\left(\mathrm{dt},{ }^{3} J=\right.$ $\left.8.2 \mathrm{~Hz},{ }^{4} J=1.3 \mathrm{~Hz}, 1 \mathrm{H}, 5-\mathrm{H}\right), 7.12\left(\mathrm{dt},{ }^{3} J=8.2 \mathrm{~Hz},{ }^{4} J=1.3 \mathrm{~Hz}, 1 \mathrm{H}, 6-\mathrm{H}\right), 7.08$ (s, 1H, 2-H), $3.90\left(\mathrm{t},{ }^{3} J=6.6 \mathrm{~Hz}, 2 \mathrm{H}, 9-\mathrm{H}\right), 3.03\left(\mathrm{t},{ }^{3} J=6.6 \mathrm{~Hz}, 2 \mathrm{H}, 8-\mathrm{H}\right) .-\mathbf{E I - M S}(70 \mathrm{eV}): m / z(\%)=161$ $\left(\left([\mathrm{M}]^{\circ+}, 25\right), 130(100), 103(6), 77\right.$ (7).

p-Hydroxybenzoesäuremethylester (77): $\mathrm{C}_{8} \mathrm{H}_{8} \mathrm{O}_{3}$ (152), weißer Feststoff, UV-löschend (254 nm). $-\boldsymbol{R}_{\boldsymbol{f}}=0.63\left(\mathrm{CH}_{2} \mathrm{Cl}_{2} / \mathrm{MeOH} 9: 1\right)$. - Keine Anfärbung. $-{ }^{1} \mathbf{H}-\mathrm{NMR}\left(\mathrm{CD}_{3} \mathrm{OD}, 300 \mathrm{MHz}\right): \delta=7.86\left(\mathrm{~d},{ }^{3} J=8.5 \mathrm{~Hz}, 2 \mathrm{H}, 2-\mathrm{H}, 6-\right.$ H), $6.81\left(\mathrm{~d},{ }^{3} \mathrm{~J}=8.5 \mathrm{~Hz}, 2 \mathrm{H}, 3-\mathrm{H}, 5-\mathrm{H}\right), 3.83\left(\mathrm{~s}, 3 \mathrm{H}, \mathrm{OCH}_{3}\right)$. - EI-MS (70 $\mathrm{eV}): m / z(\%)=152\left(\left([\mathrm{M}]^{\circ+}, 44\right), 121(100), 93(18), 65(10)\right.$.<smiles>COC(=O)c1ccc(O)cc1</smiles>

\subsection{Actinomyces sp. T421}

Der aus litoralem Sediment in Feuerland isolierte Stamm T421 bildete auf Agarplatten graues Luft- sowie gelbes Substratmycel aus. 


\subsubsection{Primärscreening}

Beim biologisches Screening des Rohextraktes gegen verschiedene Organismen (siehe Tabelle 10) konnte eine zu Stamm T426 vergleichbare cytotoxische Aktivität festgestellt werden (vgl. Kapitel 10.3.1).

\subsubsection{Kultivierung und Aufarbeitung}

Kultivierung und Aufarbeitung wurden am IBWF (Kaiserslautern) unter den in Kapitel 10.3.2 beschriebenen Bedingungen durchgeführt. Im Unterschied zu Stamm T426 erfolgte die Fermentation hier in LB-Medium bei einer Temperatur von $18^{\circ} \mathrm{C}$.

\subsubsection{Isolierung und Charakterisierung der Metaboliten}

Der Rohextrakt wurde am IBWF (Kaiserslautern) chromatographisch aufgereinigt. Es konnten $1.2 \mathrm{mg}$ trans-3-Methylthioacrylsäure (78), $1.1 \mathrm{mg}$ Benzoesäure (79) und $1.3 \mathrm{mg}$ Phenylessigsäure (80) isoliert werden.

trans-3-Methylthioacrylsäure (78): $\quad \mathrm{C}_{4} \mathrm{H}_{6} \mathrm{O}_{2} \mathrm{~S} \quad$ (118), weißer Feststoff, UV-löschend (254 nm). $-\boldsymbol{R}_{\boldsymbol{f}}=0.60\left(\mathrm{CH}_{2} \mathrm{Cl}_{2} / \mathrm{MeOH}\right.$ 9:1). - Keine Anfärbung. - ${ }^{1} \mathbf{H}-\mathbf{N M R}\left(\mathrm{CDCl}_{3}, 300 \mathrm{MHz}\right): \delta=7.88\left(\mathrm{~d},{ }^{3} \mathrm{~J}\right.$<smiles>CS/C=C/[13C](=O)O</smiles>
$=15.0 \mathrm{~Hz}, 1 \mathrm{H}, 3-\mathrm{H}), 5.66\left(\mathrm{~d},{ }^{3} J=15.0 \mathrm{~Hz}, 1 \mathrm{H}, 2-\mathrm{H}\right), 2.37(\mathrm{~s}, 3 \mathrm{H}, 5-\mathrm{H}) .-$ EI-MS $(70 \mathrm{eV}): \mathrm{m} / z$ $(\%)=118\left(\left([\mathrm{M}]^{\circ+}, 100\right), 103(91), 101(17), 100(30), 85\right.$ (15), 73 (28), 72 (23), 58 (22), 47 (17), 45 (33). - DCI-MS $\left(\mathrm{NH}_{3}\right): m / z(\%)=153\left[\mathrm{M}+\mathrm{NH}_{3}+\mathrm{NH}_{4}\right]^{+}(32), 136\left[\mathrm{M}+\mathrm{NH}_{4}\right]^{+}(100)$.

Benzoesäure (79): $\mathrm{C}_{7} \mathrm{H}_{6} \mathrm{O}_{2}$ (122), weißer Feststoff, UV-löschend (254 nm). $\boldsymbol{R}_{\boldsymbol{f}}=0.60\left(\mathrm{CH}_{2} \mathrm{Cl}_{2} / \mathrm{MeOH}\right.$ 9:1). - Keine Anfärbung. $-{ }^{1} \mathbf{H}-\mathbf{N M R}\left(\mathrm{CDCl}_{3}, 300\right.$ MHz): $\delta=8.12\left(\mathrm{~d},{ }^{3} J=7.9 \mathrm{~Hz}, 2 \mathrm{H}, 2-\mathrm{H}, 6-\mathrm{H}\right), 7.62\left(\mathrm{t},{ }^{3} J=7.2 \mathrm{~Hz}, 1 \mathrm{H}, 4-\mathrm{H}\right)$, $7.48\left(\mathrm{t},{ }^{3} J=7.5 \mathrm{~Hz}, 2 \mathrm{H}, 3-\mathrm{H}, 5-\mathrm{H}\right) .-$ EI-MS $(70 \mathrm{eV}): \mathrm{m} / z(\%)=122\left(\left([\mathrm{M}]^{\bullet+}\right.\right.$,<smiles>O=C(O)c1ccccc1</smiles>
100), 105 (90), 77 (48), 51 (18).

Phenylessigsäure (80): $\mathrm{C}_{8} \mathrm{H}_{8} \mathrm{O}_{2}$ (136), weißer Feststoff, UV-löschend (254 $\mathrm{nm}) .-\boldsymbol{R}_{\boldsymbol{f}}=0.60\left(\mathrm{CH}_{2} \mathrm{Cl}_{2} / \mathrm{MeOH} 9: 1\right)$. - Keine Anfärbung. - ${ }^{1} \mathbf{H}-\mathbf{N M R}$ $\left(\mathrm{CDCl}_{3}, 300 \mathrm{MHz}\right): \delta=7.30(\mathrm{~m}, 5 \mathrm{H}, \mathrm{Ar}-\mathrm{H}), 3.66$ (s, 2H, 2-H). - EI-MS $(70 \mathrm{eV}): m / z(\%)=136\left(\left([\mathrm{M}]^{\circ+}, 38\right), 92(18), 91(100), 65(11)\right.$.<smiles>O=C(O)Cc1ccccc1</smiles> 


\subsection{Stamm T846}

Der Stamm T846 wurde aus einer Wasserprobe des Humboldt-Stroms vor der chilenischen Küste isoliert.

\subsubsection{Primärscreening}

Im Dünnschichtchromatogramm waren einige UV-löschende Zonen zu erkennen. Nach Besprühen mit Anisaldehyd färbten sich zwei Zonen violett an. Eine weitere, stark UVlöschende und sehr polare Zone ging weder mit Anisaldehyd noch mit Ehrlichs Reagenz eine Farbreaktion ein.

\subsubsection{Kultivierung und Aufarbeitung}

Kultivierung und Aufarbeitung wurden am IBWF (Kaiserslautern) durchgeführt.

\subsubsection{Isolierung und Charakterisierung der Metaboliten}

Eine Vortrennung erfolgte am IBWF (Kaiserslautern) durch Säulenchromatographie an Kieselgel. Zwei der dabei erhaltenen Fraktionen wurden aufgrund auffälliger Zonen im Dünnschichtchromatogramm weiter bearbeitet. Nachfolgende Chromatographie an Sephadex LH$20(\mathrm{MeOH})$ und Kieselgel lieferte $3.1 \mathrm{mg}$ 3-Indolylcarbaldehyd (81), $2.8 \mathrm{mg}$ 3-Indolylcarbonsäure (33), 26.2 mg 3-Methylthiopropionsäure (82) und 12.4 mg Triazin (83).

3-Indolylcarbaldehyd (81): $\mathrm{C}_{9} \mathrm{H}_{7} \mathrm{NO}$ (145), farbloses Ö1, UVlöschend $(254 \mathrm{~nm})$. $-\boldsymbol{R}_{\boldsymbol{f}}=0.37\left(\mathrm{CH}_{2} \mathrm{Cl}_{2} / \mathrm{MeOH}\right.$ 9:1). - Anfärbeverhalten: Anisaldehyd (orange), Ehrlich (violett). $-\boldsymbol{R}_{\boldsymbol{t}}=12.0 \mathrm{~min}$ (LC-MS). $-{ }^{1} \mathbf{H}-\mathbf{N M R}\left(\left[\mathrm{D}_{6}\right]\right.$ Aceton, $\left.300 \mathrm{MHz}\right): \delta=\sim 11.2$ (br, $1 \mathrm{H}$,<smiles>O=Cc1c[nH]c2ccccc12</smiles>
$1-\mathrm{NH}), 10.02$ (s, 1H, CHO), $8.21\left(\mathrm{~d},{ }^{3} J=8.0 \mathrm{~Hz}, 1 \mathrm{H}, 7-\mathrm{H}\right), 8.19$ (s, 1H, 2-H), $7.53\left(\mathrm{~d},{ }^{3} \mathrm{~J}=8.0 \mathrm{~Hz}, 1 \mathrm{H}, 4-\mathrm{H}\right), 7.24(\mathrm{~m}, 2 \mathrm{H}, 5-\mathrm{H}, 6-\mathrm{H}) .-$ EI-MS $(70 \mathrm{eV}): \mathrm{m} / z$ $(\%)=145\left(\left([\mathrm{M}]^{\circ+}, 82\right), 144(100), 116(40), 89(54), 63(26)\right.$.

3-Methylthio-propionsäure (82): $\mathrm{C}_{4} \mathrm{H}_{8} \mathrm{O}_{2} \mathrm{~S}$ (120), farbloses Öl, UV-löschend (254 nm). $-\boldsymbol{R}_{\boldsymbol{f}}=0.51\left(\mathrm{CH}_{2} \mathrm{Cl}_{2} / \mathrm{MeOH}\right.$ 9:1). - Keine Anfärbung. - ${ }^{1} \mathbf{H}-\mathbf{N M R}\left(\mathrm{CDCl}_{3}, 300 \mathrm{MHz}\right): \delta=10.76(\mathrm{br}, 1 \mathrm{H}$,<smiles>CSCCC(=O)O</smiles>
$\mathrm{COOH}), 2.74(\mathrm{~m}, 2 \mathrm{H}, 3-\mathrm{H}), 2.66(\mathrm{~m}, 2 \mathrm{H}, 2-\mathrm{H}), 2.11\left(\mathrm{~s}, 3 \mathrm{H}, \mathrm{SCH}_{3}\right)$. 
1,3,5 Triazin (83): $\mathrm{C}_{3} \mathrm{H}_{3} \mathrm{~N}_{3}$ (81), weißer Feststoff, UV-löschend (254 nm). $\boldsymbol{R}_{\boldsymbol{f}}=0.21\left(\mathrm{CH}_{2} \mathrm{Cl}_{2} / \mathrm{MeOH} 9: 1\right)$. Keine Anfärbung. $-\boldsymbol{R}_{\boldsymbol{t}}=6.3 \mathrm{~min}(\mathrm{LC}-\mathrm{MS})$.

$-{ }^{1} \mathbf{H}-\mathbf{N M R}\left(\mathrm{CD}_{3} \mathrm{OD}, 300 \mathrm{MHz}\right): \delta=8.53$ (s, 3H, 2-H, 4-H, 6-H). $-{ }^{13} \mathbf{C}-$ NMR $\left(\mathrm{CD}_{3} \mathrm{OD}, 150.8 \mathrm{MHz}\right): \delta=170.5(\mathrm{CH}, \mathrm{C}-2, \mathrm{C}-4, \mathrm{C}-6)$.

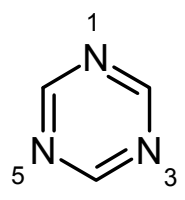

\subsection{Stamm T406}

Der aus Pinguinexkrementen isolierte Stamm T406 wuchs in Form gelber, feiner Kolonien und zeigte psychrophiles Verhalten.

\subsubsection{Primärscreening}

Im Dünnschichtchromatogramm waren mehrere gelbe bzw. UV-löschende Zonen auffällig, von denen eine nach Besprühen mit Anisaldehyd eine dunkelviolette Farbreaktion einging. Das biologische Screening des Rohextraktes gegen verschiedenen Organismen (Tabelle 10) ergab die bereits bei den Stämmen T426 (Kapitel 10.3.1) und T421-A (Kapitel 10.4.1) auffällige Cytotoxizität gegenüber bestimmten Zelllinien. Weiterhin wies Stamm T406 eine Aktivität gegen Nematoden auf.

\subsubsection{Kultivierung und Aufarbeitung}

Kultivierung und Aufarbeitung wurden am IBWF (Kaiserslautern) unter den in Kapitel 10.3.2 beschriebenen Bedingungen durchgeführt. Die Fermentation erfolgte in B-Medium bei $4{ }^{\circ} \mathrm{C}$, wobei sich $3.1 \mathrm{~g}$ Rohextrakt ergaben.

\subsubsection{Isolierung und Charakterisierung der Metaboliten}

Der Rohextrakt wurde mittels Säulenchromatographie an Kieselgel unter Verwendung eines Stufengradienten aus Dichlormethan/Methanol $\left(\mathrm{CH}_{2} \mathrm{Cl}_{2}, \mathrm{CH}_{2} \mathrm{Cl}_{2} / \mathrm{MeOH}\right.$ 99:1, $\mathrm{CH}_{2} \mathrm{Cl}_{2} / \mathrm{MeOH}$ 98:2, $\mathrm{CH}_{2} \mathrm{Cl}_{2} / \mathrm{MeOH}$ 97:3, $\mathrm{CH}_{2} \mathrm{Cl}_{2} / \mathrm{MeOH}$ 95:5, $\mathrm{CH}_{2} \mathrm{Cl}_{2} / \mathrm{MeOH}$ 9:1) getrennt. Anschließende chromatographische Reinigung an Sephadex $\mathrm{LH} 20\left(\mathrm{CH}_{2} \mathrm{Cl}_{2} / \mathrm{MeOH} 3: 2\right.$ und $\left.\mathrm{MeOH}\right)$ sowie Flashchromatographie an Kieselgel $\left(\mathrm{CH}_{2} \mathrm{Cl}_{2} / \mathrm{MeOH}\right)$ ergaben $1.9 \mathrm{mg}$ 2-(Indol-3-ylmethyl)indol-3-ylethanol (84), $3.6 \mathrm{mg}$ Ubichinon Q7 (87), $0.2 \mathrm{mg}$ SS-43405e (88), $1.3 \mathrm{mg}$ 2-(3- 
Indolyl)ethylacetat (89), $1.5 \mathrm{mg}$ Cyclo(Dehydroala-Leu) (90), $18.1 \mathrm{mg}$ Phenylethanol (91) und $8.4 \mathrm{mg}$ 2-(3-Indolyl)ethanol (76).

\section{2-(Indol-3-ylmethyl)-indol-3-ylethanol}

$\mathrm{C}_{19} \mathrm{H}_{18} \mathrm{~N}_{2} \mathrm{O}$ (290), farbloser Feststoff, UV-löschend $(254 \mathrm{~nm}) .-\boldsymbol{R}_{\boldsymbol{f}}=0.75\left(\mathrm{CH}_{2} \mathrm{Cl}_{2} / \mathrm{MeOH} 9: 1\right) .-$ Anfärbeverhalten: Anisaldehyd (violett). $-\boldsymbol{R}_{t}=16.6 \mathrm{~min}$ (LC-MS). $-{ }^{1} \mathbf{H}-\mathbf{N M R}\left(\mathrm{CD}_{3} \mathrm{OD}, 300 \mathrm{MHz}\right): \delta=7.46$ (d br, $\left.{ }^{3} J=7.7 \mathrm{~Hz}, 1 \mathrm{H}, 4^{\prime}-\mathrm{H}\right), 7.43$ (d br, ${ }^{3} J=8.0 \mathrm{~Hz}$, $1 \mathrm{H}, 4$ '’-H), 7.31 (d br, ${ }^{3} J=8.2 \mathrm{~Hz}, 1 \mathrm{H}, 7$ ' $\left.-\mathrm{H}\right), 7.20$ (d br, ${ }^{3} J=7.6 \mathrm{~Hz}, 1 \mathrm{H}, 7$ '-H), 7.05 (t br, ${ }^{3} J=7.6 \mathrm{~Hz}, 1 \mathrm{H}, 6$ ' '-H), 6.97 (m, 1H, 6'-H), 6.96 (m, 1H, 2'’-H), 6.94 (m, 1H, 5'-H), 6.92 (m, 1H, 5' -H), 4.22 (s, 2H, 8'-H), 3.69 (t, $\left.{ }^{3} J=7.6 \mathrm{~Hz}, 2 \mathrm{H}, 1-\mathrm{H}\right), 3.04\left(\mathrm{t},{ }^{3} J=7.6 \mathrm{~Hz}, 2 \mathrm{H}, 2-\mathrm{H}\right)$. ${ }^{13}$ C-NMR (CD $\left.{ }_{3} \mathrm{OD}, 150.8 \mathrm{MHz}\right): \delta=138.3\left(\mathrm{C}_{\mathrm{q}}, \mathrm{C}-7\right.$ ' 'a), $137.2\left(\mathrm{C}_{\mathrm{q}}, \mathrm{C}-7\right.$ 'a $), 136.6\left(\mathrm{C}_{\mathrm{q}}, \mathrm{C}-2\right.$ '), 130.1 (C $\mathrm{q}_{\mathrm{q}}, \mathrm{C}-3$ 'a), 128.6 (C $\mathrm{q}, \mathrm{C}-3$ '’a), 123.8 (CH, C-2'’), 122.3 (CH, C-6'’), 121.4 (CH, C-6'), 119.6 (CH, C-5'), 119.41 (CH, C-4'’), 119.37 (CH, C-5'), $118.6\left(\mathrm{CH}, \mathrm{C}-4\right.$ '), $114.0\left(\mathrm{C}_{\mathrm{q}}, \mathrm{C}-\right.$ 3'’), 112.2 (CH, C-7'’), $111.6\left(\mathrm{CH}, \mathrm{C}-7\right.$ '), $108.0\left(\mathrm{C}_{\mathrm{q}}, \mathrm{C}-3\right.$ '), $63.7\left(\mathrm{CH}_{2}, \mathrm{C}-1\right), 29.0\left(\mathrm{CH}_{2}, \mathrm{C}-2\right)$, $23.3\left(\mathrm{CH}_{2}, \mathrm{C}-8^{\prime}\right)$. - EI-MS (70 eV): $m / z(\%)=290\left(\left([\mathrm{M}]^{\circ+}, 33\right), 259(100), 130(37)\right.$. - DCIMS $\left(\mathrm{NH}_{3}\right): m / z(\%)=582[2 \mathrm{M}+\mathrm{H}]^{+}(0.5), 308\left[\mathrm{M}+\mathrm{NH}_{4}\right]^{+}(14), 291[\mathrm{M}+\mathrm{H}]^{+}(100) .-\mathbf{U V}$ $(\mathrm{MeOH}): \lambda_{\max }(\lg \varepsilon)=224(4.08), 282(2.91), 385(2.55) \mathrm{nm}$.

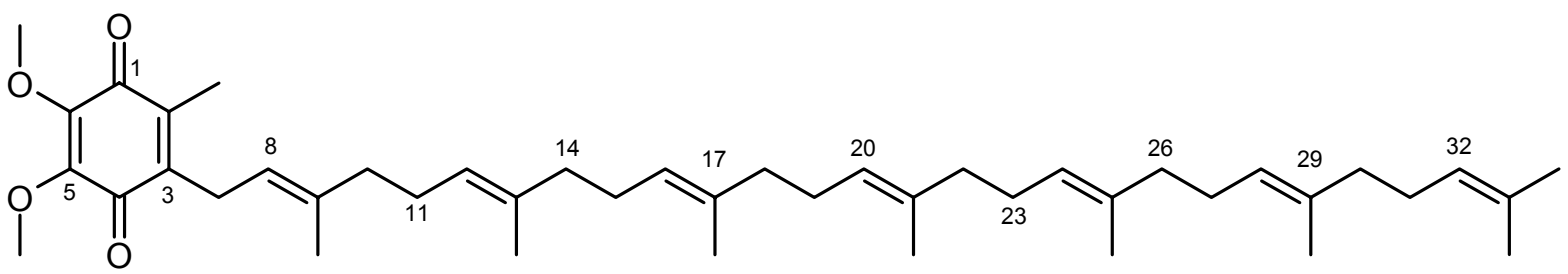

Ubichinon Q7 (87): $\mathrm{C}_{44} \mathrm{H}_{66} \mathrm{O}_{4}$ (658), gelbes Öl, UV-löschend (254 nm). - $\boldsymbol{R}_{\boldsymbol{f}}=0.95$ $\left(\mathrm{CH}_{2} \mathrm{Cl}_{2} / \mathrm{MeOH}\right.$ 95:5). - Anfärbeverhalten: Anisaldehyd (violett). - ${ }^{1} \mathbf{H}-\mathbf{N M R}\left(\mathrm{CDCl}_{3}, 300\right.$ MHz): $\delta=5.10-5.04$ (m, 6H, 12-, 16-, 20-, 24-, 28-, 32-H), 4.91 (t, 1H, 8-H), 3.97 (s, 3H, 5$\left.\mathrm{OCH}_{3}\right), 3.96\left(\mathrm{~s}, 3 \mathrm{H}, 6-\mathrm{OCH}_{3}\right), 3.16(\mathrm{~d}, 2 \mathrm{H}, 7-\mathrm{H}), 2.05-1.94$ (m, 24H, 10-, 11-, 14-, 15-, 18-, 19-, 22-, 23-, 26-, 27-, 30-, 31-H), 1.99 (s, 3H, 2- $\mathrm{CH}_{3}$ ), 1.71 (s, 3H, 9- $\left.\mathrm{CH}_{3}\right), 1.65$ (s, 3H, 13$\left.\mathrm{CH}_{3}\right), 1.56$ (s, 12H, 17-, 21-, 25-, 29- $\left.\mathrm{CH}_{3}\right), 1.55$ (s, 6H, 33- $\left.\mathrm{CH}_{3}, 34-\mathrm{H}\right)$. 
SS-43405e (88): $\mathrm{C}_{22} \mathrm{H}_{18} \mathrm{O}_{5}$ (362), gelber Feststoff, UVlöschend (254 nm). - $\boldsymbol{R}_{\boldsymbol{f}}=0.88\left(\mathrm{CH}_{2} \mathrm{Cl}_{2} / \mathrm{MeOH} 9: 1\right)$. Keine Anfärbung. $-\boldsymbol{R}_{\boldsymbol{t}}=24.7 \mathrm{~min}$ (LC-MS). $-{ }^{\mathbf{1}} \mathbf{H}-\mathbf{N M R}$ $\left(\mathrm{CDCl}_{3}, 600 \mathrm{MHz}\right): \delta=12.92(\mathrm{~s}, 1 \mathrm{H}, \mathrm{OH}), 8.03$ (s, 1H, 6H), $7.80\left(\mathrm{~d},{ }^{3} J=7.6 \mathrm{~Hz}, 1 \mathrm{H}, 8-\mathrm{H}\right), 7.65\left(\mathrm{t},{ }^{3} J=8.2 \mathrm{~Hz}, 1 \mathrm{H}\right.$, 9-H), 7.34 (d, $\left.{ }^{3} J=8.5 \mathrm{~Hz}, 1 \mathrm{H}, 10-\mathrm{H}\right), 6.24$ (s, 1H, 3-H), 3.00 (s, 3H, 5-H), 2.70 (sextett, ${ }^{3} J=7.0 \mathrm{~Hz}, 1 \mathrm{H}, 13-\mathrm{H}$ ),<smiles>CCC(C)c1cc(=O)c2c(C)cc3c(c2o1)C(=O)c1c(O)cccc1C3=O</smiles>
$1.95\left(\mathrm{dq},{ }^{2} J=13.8 \mathrm{~Hz},{ }^{3} J=7.6 \mathrm{~Hz}, 1 \mathrm{H}, 14-\mathrm{H}_{\mathrm{A}}\right), 1.76$ (dq, $\left.{ }^{2} J=13.8 \mathrm{~Hz},{ }^{3} J=7.0 \mathrm{~Hz}, 1 \mathrm{H}, 14-\mathrm{H}_{\mathrm{B}}\right), 1.41$ (d, 3H, 13- $\left.\mathrm{CH}_{3}\right), 0.96$ (t, 3H, 15-H). - (+)-ESIMS: $m / z(\%)=747[2 \mathrm{M}+\mathrm{Na}]^{+}(100), 363[\mathrm{M}+\mathrm{H}]^{+}(41)$. - (-)-ESI-MS: $m / z(\%)=745[2 \mathrm{M}-$ $2 \mathrm{H}+\mathrm{Na}]^{-}(100), 361[\mathrm{M}-\mathrm{H}]^{-}(17)$.

2-(3-Indolyl)ethylacetat (89): $\mathrm{C}_{12} \mathrm{H}_{13} \mathrm{NO}_{2}$ (203), weißer Feststoff, UV-löschend $(254 \mathrm{~nm}) . \quad-\boldsymbol{R}_{\boldsymbol{f}}=0.81$ $\left(\mathrm{CH}_{2} \mathrm{Cl}_{2} / \mathrm{MeOH}\right.$ 9:1). - Anfärbeverhalten: Anisaldehyd (rot), Ehrlich (violett). $-\boldsymbol{R}_{\boldsymbol{t}}=15.7 \mathrm{~min}$ (LC-MS). $-{ }^{\mathbf{1}} \mathbf{H}-$<smiles>CC(=O)OCCc1c[nH]c2ccccc12</smiles>
NMR $\left(\mathrm{CDCl}_{3}, 300 \mathrm{MHz}\right): \delta=8.00(\mathrm{br}, 1 \mathrm{H}, 1-\mathrm{NH}), 7.64\left(\mathrm{~d},{ }^{3} J=7.8 \mathrm{~Hz}, 1 \mathrm{H}, 4-\mathrm{H}\right), 7.38\left(\mathrm{~d},{ }^{3} J\right.$ $=7.9 \mathrm{~Hz}, 1 \mathrm{H}, 7-\mathrm{H}), 7.20\left(\mathrm{t},{ }^{3} J=7.8 \mathrm{~Hz}, 1 \mathrm{H}, 5-\mathrm{H}\right), 7.13\left(\mathrm{t},{ }^{3} J=7.9 \mathrm{~Hz}, 1 \mathrm{H}, 6-\mathrm{H}\right), 7.06\left(\mathrm{~d},{ }^{3} J=\right.$ $7.2 \mathrm{~Hz}, 1 \mathrm{H}, 2-\mathrm{H}), 4.35$ (t, $\left.{ }^{3} \mathrm{~J}=7.2 \mathrm{~Hz}, 2 \mathrm{H}, 9-\mathrm{H}\right), 3.10$ (t, $\left.{ }^{3} \mathrm{~J}=7.2 \mathrm{~Hz}, 2 \mathrm{H}, 8-\mathrm{H}\right), 2.05$ (s, 3H, 12-H). - EI-MS (70 eV): $m / z(\%)=203\left(\left([\mathrm{M}]^{\circ+}, 28\right), 143(100), 130(90)\right.$.

Cyclo(Dehydroala-Leu) (90): $\quad \mathrm{C}_{9} \mathrm{H}_{14} \mathrm{~N}_{2} \mathrm{O}_{2} \quad$ (182), weißer Feststoff, UV-löschend (254 nm). $-\boldsymbol{R}_{\boldsymbol{f}}=0.51\left(\mathrm{CH}_{2} \mathrm{Cl}_{2} / \mathrm{MeOH}\right.$ 9:1). - Anfärbeverhalten: Anisaldehyd (orange), Ehrlich (orange). $-\boldsymbol{R}_{\boldsymbol{t}}=9.7 \mathrm{~min}(\mathrm{LC}-\mathrm{MS}) .-{ }^{1} \mathbf{H}-\mathrm{NMR}\left(\left[\mathrm{D}_{6}\right] \mathrm{DMSO}, 600 \mathrm{MHz}\right): \delta=$ 10.47 (br, 1H, 1-NH), 8.40 (s br, 1H, 4-NH), 5.18 (s, 1H, 11- $\left.\mathrm{H}_{\mathrm{A}}\right)$,<smiles>C=C1NC(=O)C(CC(C)C)NC1=O</smiles>
$4.79\left(\mathrm{~s}, 1 \mathrm{H}, 11-\mathrm{H}_{\mathrm{B}}\right), 3.96\left(\mathrm{t},{ }^{3} \mathrm{~J}=8.0 \mathrm{~Hz}, 1 \mathrm{H}, 6-\mathrm{H}\right), 1.80$ (septett, $\left.{ }^{3} J=6.7 \mathrm{~Hz}, 1 \mathrm{H}, 8-\mathrm{H}\right), 1.58(\mathrm{~m}, 2 \mathrm{H}, 7-\mathrm{H}), 0.86\left(\mathrm{~d},{ }^{3} \mathrm{~J}=6.5 \mathrm{~Hz}, 6 \mathrm{H}, 9-\mathrm{H}, 10-\mathrm{H}\right) .-{ }^{13} \mathbf{C}-\mathbf{N M R}$ ([D $]$ DMSO, $150.8 \mathrm{MHz}): \delta=166.4\left(\mathrm{C}_{\mathrm{q}}, \mathrm{C}-5\right), 158.2\left(\mathrm{C}_{\mathrm{q}}, \mathrm{C}-2\right), 134.7\left(\mathrm{C}_{\mathrm{q}}, \mathrm{C}-3\right), 98.9\left(\mathrm{CH}_{2}\right.$, C-11), 53.7 (CH, C-6), $43.5\left(\mathrm{CH}_{2}, \mathrm{C}-7\right), 23.3$ (CH, C-8), $22.6\left(\mathrm{CH}_{3}, \mathrm{C}-9\right), 22.1\left(\mathrm{CH}_{3}, \mathrm{C}-10\right)$.

Phenylethanol (91): $\mathrm{C}_{8} \mathrm{H}_{10} \mathrm{O}$ (122), farblose Flüssigkeit, UVlöschend (254 nm). - $\boldsymbol{R}_{\boldsymbol{f}}=0.63\left(\mathrm{CH}_{2} \mathrm{Cl}_{2} / \mathrm{MeOH} 9: 1\right)$. - Keine Anfärbung. $-{ }^{1} \mathbf{H}-\mathbf{N M R}\left(\mathrm{CDCl}_{3}, 300 \mathrm{MHz}\right): \delta=7.32\left(\mathrm{~m}, 2 \mathrm{H}, 2{ }^{\prime}-\mathrm{H}\right.$,<smiles>OCCc1ccccc1</smiles>
6'-H), 7.25 (m, 3H, 3'-H, 4'-H, 5'-H), 3.83 (t, ${ }^{3} J=7.9$ Hz, 2H, 1- 
H), $2.86\left(\mathrm{t},{ }^{3} \mathrm{~J}=7.9 \mathrm{~Hz}, 2 \mathrm{H}, 2-\mathrm{H}\right)$. - EI-MS $(70 \mathrm{eV}): m / z(\%)=122\left(\left([\mathrm{M}]^{\circ+}, 34\right), 92(58), 91\right.$ (100), 65 (18).

\subsection{Streptomyces flavogriseus ACT7651}

Der Stamm wurde aus litoralem Sediment isoliert, das aus der Nähe von Punta Arenas (Feuerland) stammte.

\subsubsection{Primärscreening}

Der Rohextrakt von Stamm ACT2298 fiel im Dünnschichtchromatogramm durch mehrere UV-löschende Zonen auf, welche sich beim Besprühen mit Anisaldehyd blau färbten.

Tabelle 11 Aktivität des Rohextraktes von Stamm ACT2298 im biologischen Screening.

\begin{tabular}{|l|c|}
\hline \multicolumn{1}{|c|}{ Testkeim } & Hemmhof $\varnothing[\mathrm{mm}]$ \\
\hline Bacillus subtilis & 30 \\
\hline Staphylococcus aureus & 25 \\
\hline Escherichia coli & 30 \\
\hline Candida albicans & 17 \\
\hline Mucor miehei (Tü 284) & 11 \\
\hline Chlorella vulgaris & 12 \\
\hline Chlorella sorokiniana & 13 \\
\hline Scenedesmus subspicatus & 13 \\
\hline
\end{tabular}

\subsubsection{Kultivierung und Aufarbeitung}

Die Kultivierung erfolgte im 15 I Schüttelkultur-Maßstab in 1 I Erlenmeyerkolben, welche mit jeweils $200 \mathrm{ml}$ M2-Medium befüllt und mit einem $1 \mathrm{~cm}^{2}$ großen Stück einer gut bewachsenen Agarplatte beimpft wurden. Die Fermentation wurde auf einem Linearschüttler bei $28^{\circ} \mathrm{C}$ für vier Tage durchgeführt.

Die Aufarbeitung der Kulturen erfolgte zunächst durch Korrektur des pH-Wertes auf 5.0, Zusatz von Celite und Filtration. Das Kulturfiltrat wurde an XAD-16 adsorbiert, das Adsor- 
berharz anschließend mit 101 Wasser gewaschen und mit 5 I Methanol eluiert. Das nach dieser Behandlung vorliegende Kulturfiltrat wurde zusätzlich mit Essigester extrahiert und führte zu $50 \mathrm{mg}$ hellgelbem Extrakt. Der mit Celite vermischte Zellrückstand wurde dreimal mit Essigester und zweimal mit Aceton unter Ultraschall-Behandlung extrahiert. Die vereinigten organischen Phasen ergaben nach Entfernen des Lösungsmittels 6.8 g Rohextrakt.

\subsubsection{Isolierung und Charakterisierung der Metaboliten}

Der Rohextrakt wurde mittels Säulenchromatographie an Kieselgel mit einem Dichlormethan/ Methanol-Gradienten (1000 ml $\mathrm{CH}_{2} \mathrm{Cl}_{2} / \mathrm{MeOH}$ 95:5 und $2000 \mathrm{ml} \mathrm{CH}_{2} \mathrm{Cl}_{2} / \mathrm{MeOH}$ 9:1) getrennt. Anschließende chromatographische Reinigung an Sephadex LH20 $\left(\mathrm{CH}_{2} \mathrm{Cl}_{2} / \mathrm{MeOH}\right.$ 3:2 und $\mathrm{MeOH})$ sowie präparative Dickschichtchromatographie an Kieselgel $\left(\mathrm{CH}_{2} \mathrm{Cl}_{2} / \mathrm{MeOH}\right.$ 9:1) ergaben $67.7 \mathrm{mg}$ 2-Desoxyuridin (92), $45.1 \mathrm{mg}$ 2-Desoxythymidin (93), $10.9 \mathrm{mg}$ 2'-OMethyluridin (94), $54 \mathrm{mg}$ Enterocin (95), $43 \mathrm{mg}$ 5-Deoxyenterocin (96) und $33 \mathrm{mg}$ transZimtsäureamid (99). Der Essigester-Extrakt des Kulturfiltrates wurde mittels Säulenchromatographie an Kieselgel $\left(\mathrm{CH}_{2} \mathrm{Cl}_{2} / \mathrm{MeOH}\right.$ 95:5) getrennt. Weitere Reinigung an Sephadex LH20 (MeOH) lieferte $4.3 \mathrm{mg}$ Venturicidin A (100).

2-Desoxyuridin (92): $\mathrm{C}_{9} \mathrm{H}_{12} \mathrm{~N}_{2} \mathrm{O}_{5}$ (228), weißer Feststoff, UVlöschend $(254 \mathrm{~nm})$. $\quad-\boldsymbol{R}_{\boldsymbol{f}}=0.05\left(\mathrm{CH}_{2} \mathrm{Cl}_{2} / \mathrm{MeOH}\right.$ 9:1). Anfärbeverhalten: Anisaldehyd (blau), schwach Ehrlich (braun). $\boldsymbol{R}_{\boldsymbol{t}}=2.9 \min (\mathrm{LC}-\mathrm{MS}) .-{ }^{1} \mathbf{H}-\mathrm{NMR}\left(\mathrm{CD}_{3} \mathrm{OD}, 300 \mathrm{MHz}\right): \delta=7.97$ $\left(\mathrm{d},{ }^{3} J=8.3 \mathrm{~Hz}, 1 \mathrm{H}, 6-\mathrm{H}\right), 6.26\left(\mathrm{t},{ }^{3} J=6.6 \mathrm{~Hz}, 1 \mathrm{H}, 1^{\prime}-\mathrm{H}\right), 5.69\left(\mathrm{~d},{ }^{3} J\right.$ $=7.9 \mathrm{~Hz}, 1 \mathrm{H}, 5-\mathrm{H}), 4.37\left(\mathrm{dt},{ }^{3} J=6.0 \mathrm{~Hz}, 3.4 \mathrm{~Hz}, 1 \mathrm{H}, 3^{\prime}-\mathrm{H}\right), 3.92$ (q, $\left.{ }^{3} J=3.4 \mathrm{~Hz}, 1 \mathrm{H}, 4^{\prime}-\mathrm{H}\right), 3.76,3.72\left(\mathrm{ABX},{ }^{2} J=12.0 \mathrm{~Hz},{ }^{3} J=3.3\right.$

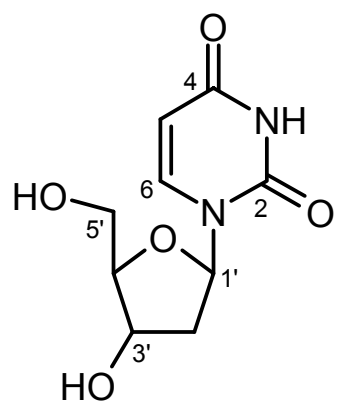
Hz, 2H, 5'-H), 2.22 (m, 2H, 2'-H). - (+)-ESI-MS: $m / z(\%)=251$ (95). - (-)-ESI-MS: $m / z(\%)$ $=227(20)$. EI-MS $(70 \mathrm{eV}): m / z(\%)=228\left(\left([\mathrm{M}]^{\circ+}, 2\right), 117\right.$ (100), $112(29), 96(15), 73(52)$, 69 (51), 45 (39).

2-Desoxythymidin (93): $\mathrm{C}_{10} \mathrm{H}_{14} \mathrm{~N}_{2} \mathrm{O}_{5}$ (242), weißer Feststoff, UVlöschend $(254 \mathrm{~nm})$. $\quad-\boldsymbol{R}_{\boldsymbol{f}}=0.07 \quad\left(\mathrm{CH}_{2} \mathrm{Cl}_{2} / \mathrm{MeOH}\right.$ 9:1). Anfärbeverhalten: Anisaldehyd (blau), Ehrlich (schwach braun). $\boldsymbol{R}_{\boldsymbol{t}}=3.9 \min (\mathrm{LC}-\mathrm{MS}) .-{ }^{\mathbf{1}} \mathbf{H}-\mathbf{N M R}\left(\mathrm{CD}_{3} \mathrm{OD}, 300 \mathrm{MHz}\right): \delta=7.80$ (s, 1H, 6-H), 6.28 (t, ${ }^{3} J=7.0 \mathrm{~Hz}, 1 \mathrm{H}, 1$ '-H), 4.39 (m, 1H, 3'-H),<smiles>Cc1cn([C]2CC(O)[C@@H](CO)O2)c(=O)[nH]c1=O</smiles> 
3.90 (q, $\left.{ }^{3} J 3.4 \mathrm{~Hz}, 1 \mathrm{H}, 4^{\prime}-\mathrm{H}\right), 3.78,3.72\left(\mathrm{~m},{ }^{2} J=12.1 \mathrm{~Hz},{ }^{3} J=3.1 \mathrm{~Hz}, 2 \mathrm{H}, 5^{\prime}-\mathrm{H}\right), 2.23$ (m, 2H, 2'-H), $1.86\left(\mathrm{~s}, 5-\mathrm{CH}_{3}\right) .-(+)$-ESI-MS: $m / z(\%)=507[2 \mathrm{M}+\mathrm{Na}]^{+}(68), 265[\mathrm{M}+\mathrm{Na}]^{+}$ (100), $243[\mathrm{M}+\mathrm{H}]^{+}(2)$. - (-)-ESI-MS: $m / z(\%)=287\left[_{\mathrm{M}+\mathrm{HCOO}^{-}}(100), 241[\mathrm{M}-\mathrm{H}]^{-}(6)\right.$.

2'-O-Methyluridin (94): $\mathrm{C}_{10} \mathrm{H}_{14} \mathrm{~N}_{2} \mathrm{O}_{6}$ (258), weißer Feststoff, UVlöschend $(254 \mathrm{~nm})$. $\quad-\boldsymbol{R}_{\boldsymbol{f}}=0.09 \quad\left(\mathrm{CH}_{2} \mathrm{Cl}_{2} / \mathrm{MeOH}\right.$ 9:1). Anfärbeverhalten: Anisaldehyd (grün), Ehrlich (schwach braun). $\boldsymbol{R}_{\boldsymbol{t}}=3.6 \min (\mathrm{LC}-\mathrm{MS}) .-{ }^{1} \mathbf{H}-\mathbf{N M R}\left(\mathrm{CD}_{3} \mathrm{OD}, 300 \mathrm{MHz}\right): \delta=8.08$ (d, $\left.{ }^{3} J=7.9 \mathrm{~Hz}, 1 \mathrm{H}, 6-\mathrm{H}\right), 5.93\left(\mathrm{~d},{ }^{3} J=3.4 \mathrm{~Hz}, 1 \mathrm{H}, 1^{\prime}-\mathrm{H}\right), 5.69$ (d, ${ }^{3} J$ $=8.2 \mathrm{~Hz}, 1 \mathrm{H}, 5-\mathrm{H}), 4.24\left(\mathrm{t},{ }^{3} \mathrm{~J}=6.0 \mathrm{~Hz}, 1 \mathrm{H}, 3^{\prime}-\mathrm{H}\right), 3.97\left(\mathrm{dt},{ }^{3} J=\right.$ 6.0, 3.0 Hz, 1H, 4'-H), 3.87 (dd, $\left.{ }^{3} J=12.3,3.0 \mathrm{~Hz}, 1 \mathrm{H}, 5^{\prime}-\mathrm{H}_{\mathrm{B}}\right) 3.84$

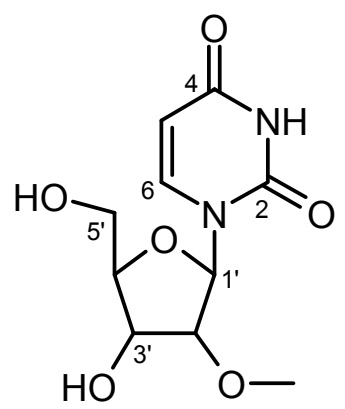
$\left(\mathrm{dd},{ }^{3} J=6.0,3.5 \mathrm{~Hz}, 1 \mathrm{H}, 2^{\prime}-\mathrm{H}\right), 3.74\left(\mathrm{dd},{ }^{3} J=12.3,3.0 \mathrm{~Hz}, 1 \mathrm{H}, 5^{\prime}-\mathrm{H}_{\mathrm{A}}\right), 3.51$ (s, 3H, 2'$\left.\mathrm{OCH}_{3}\right)$.

Enterocin (95): $\mathrm{C}_{22} \mathrm{H}_{20} \mathrm{O}_{10}$ (444), weißer Feststoff, UVlöschend $(254 \mathrm{~nm})$. $-\boldsymbol{R}_{\boldsymbol{f}}=0.21\left(\mathrm{CH}_{2} \mathrm{Cl}_{2} / \mathrm{MeOH} 9: 1\right)$. Anfärbeverhalten: Anisaldehyd (blau), Ehrlich (hellrot). $\boldsymbol{R}_{\boldsymbol{t}}=8.4 \min (\mathrm{LC}-\mathrm{MS}) .-{ }^{\mathbf{1}} \mathbf{H}-\mathbf{N M R}\left(\mathrm{CDCl}_{3}, 300 \mathrm{MHz}\right): \delta=$ $7.84\left(\mathrm{~d},{ }^{3} J=7.7 \mathrm{~Hz}, 2 \mathrm{H}, 18-\mathrm{H}, 22-\mathrm{H}\right), 7.51\left(\mathrm{t},{ }^{3} J=7.5 \mathrm{~Hz}\right.$, 1H, 20-H), 7.39 (t, ${ }^{3} J=7.4$ Hz, 2H, 19-H, 21-H), 6.30 (d, $\left.{ }^{4} J=2.2 \mathrm{~Hz}, 1 \mathrm{H}, 11-\mathrm{H}\right), 5.42\left(\mathrm{~d},{ }^{4} J=2.2 \mathrm{~Hz}, 1 \mathrm{H}, 13-\mathrm{H}\right)$, 4.77 (m, 1H, 6-H), 4.69 (br, 1H, OH), 4.64 (s, 1H, 3-H),

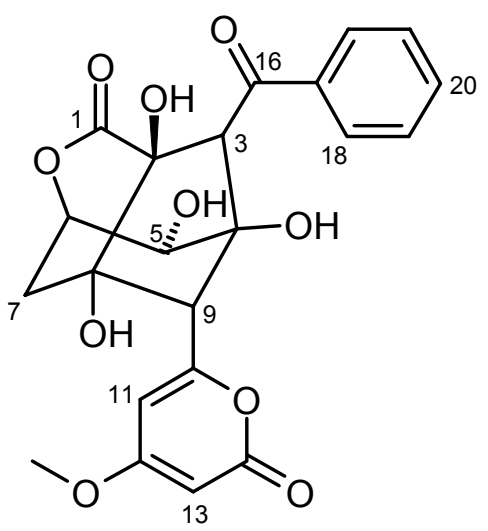
$4.61\left(\mathrm{~d},{ }^{3} J=4.5 \mathrm{~Hz}, 1 \mathrm{H}, 5-\mathrm{H}\right), 4.57$ (br, 1H, OH), 4.42 (d, $\left.{ }^{3} J=2.5 \mathrm{~Hz}, 1 \mathrm{H}, 9-\mathrm{H}\right), 4.35$ (br, $\left.1 \mathrm{H}, \mathrm{OH}\right), 3.75(\mathrm{~s}, 3 \mathrm{H}, 15-\mathrm{H}), 2.53\left(\mathrm{dd},{ }^{2} J=14.7 \mathrm{~Hz},{ }^{3} J=3.1\right.$ Hz, $\left.1 \mathrm{H}, 7-\mathrm{H}_{\mathrm{ax}}\right), 1.84\left(\mathrm{dd},{ }^{2} J=14.7 \mathrm{~Hz},{ }^{3} J=2.6 \mathrm{~Hz}, 1 \mathrm{H}, 7-\mathrm{H}_{\text {äq }}\right) \cdot-(+)$-ESI-MS: $m / z(\%)=911$ $[2 \mathrm{M}+\mathrm{Na}]^{+}(100), 467[\mathrm{M}+\mathrm{Na}]^{+}(16), 445[\mathrm{M}+\mathrm{H}]^{+}(8)$.

\section{5-Desoxyenterocin (96):}

$\mathrm{C}_{22} \mathrm{H}_{20} \mathrm{O}_{9}$ (428), weißer Feststoff, UV-löschend (254 nm). $\boldsymbol{R}_{\boldsymbol{f}}=0.35\left(\mathrm{CH}_{2} \mathrm{Cl}_{2} / \mathrm{MeOH} 9: 1\right)$. - Keine Anfärbung. $-\boldsymbol{R}_{\boldsymbol{t}}=$ $10.2 \min (\mathrm{LC}-\mathrm{MS}) .-{ }^{\mathbf{1}} \mathbf{H}-\mathbf{N M R}\left(\mathrm{CDCl}_{3}, 300 \mathrm{MHz}\right): \delta=$ $7.84\left(\mathrm{~d},{ }^{3} \mathrm{~J}=8.0 \mathrm{~Hz}, 2 \mathrm{H}, 18-\mathrm{H}, 22-\mathrm{H}\right), 7.60\left(\mathrm{t},{ }^{3} J=7.1 \mathrm{~Hz}\right.$, 1H, 20-H), 7.48 (t, $\left.{ }^{3} J=7.4 \mathrm{~Hz}, 2 \mathrm{H}, 19-\mathrm{H}, 21-\mathrm{H}\right), 6.28\left(\mathrm{~d},{ }^{4} J\right.$ $=1.9 \mathrm{~Hz}, 1 \mathrm{H}, 11-\mathrm{H}), 5.48\left(\mathrm{~d},{ }^{4} J=1.9 \mathrm{~Hz}, 1 \mathrm{H}, 13-\mathrm{H}\right), 4.99$ (m, 1H, 6-H), 4.21 (s, 1H, 9-H), 3.99 (s, 1H, 3-H), 3.80 (s,

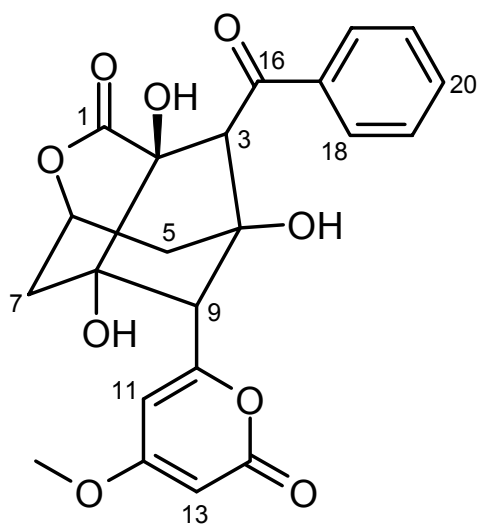


$3 \mathrm{H}, 15-\mathrm{H}), 2.83\left(\mathrm{dd},{ }^{2} J=15.1 \mathrm{~Hz},{ }^{3} J=4.0 \mathrm{~Hz}, 1 \mathrm{H}, 5-\mathrm{H}_{\mathrm{ax}}\right), 2.71\left(\mathrm{dd},{ }^{2} J=14.4 \mathrm{~Hz},{ }^{3} J=2.8 \mathrm{~Hz}\right.$, $\left.1 \mathrm{H}, 7-\mathrm{H}_{\mathrm{ax}}\right), 2.16\left(\mathrm{t},{ }^{3} J=15.1 \mathrm{~Hz}, 1 \mathrm{H}, 5-\mathrm{H}_{\mathrm{äq}}\right), 1.85\left(\mathrm{t},{ }^{3} J=14.4 \mathrm{~Hz}, 1 \mathrm{H}, 7-\mathrm{H}_{\mathrm{äq}}\right) .-(+)-\mathbf{E S I}-\mathbf{M S}$ : $m / z(\%)=879[2 \mathrm{M}+\mathrm{Na}]^{+}(100), 857[2 \mathrm{M}+\mathrm{H}]^{+}(5), 429[\mathrm{M}+\mathrm{H}]^{+}(23)$.

trans-Zimtsäureamid (99): $\mathrm{C}_{9} \mathrm{H}_{8} \mathrm{O}_{2}$ (147), weißer Feststoff, UV-löschend (254 nm). $-\boldsymbol{R}_{\boldsymbol{f}}=0.39\left(\mathrm{CH}_{2} \mathrm{Cl}_{2} / \mathrm{MeOH}\right.$ 9:1). Anfärbeverhalten: Anisaldehyd (hellrot), Ehrlich (gelb). $-\boldsymbol{R}_{\boldsymbol{t}}=$ $11.1 \min (\mathrm{LC}-\mathrm{MS}) .-{ }^{\mathbf{1}} \mathbf{H}-\mathbf{N M R}$ (d-Aceton, $\left.300 \mathrm{MHz}\right): \delta=7.58$<smiles>NC(=O)/C=C/c1ccccc1</smiles>
(m, 2H, 2'-H, 6'-H), 7.57 (d, $\left.{ }^{3} J=15.7 \mathrm{~Hz}, 1 \mathrm{H}, 2-\mathrm{H}\right), 7.38$ (m, 3H, 3'-H, 4'-H, 5'-H), 7.13 (br, 1H, NH), 6.74 (d, ${ }^{3} J=15.7$ Hz, 1H, 3-H), 6.68 (br, 1H, NH). - EI-MS $(70 \mathrm{eV}): m / z(\%)=146\left(\left([\mathrm{M}]^{\circ+}, 100\right), 131\right.$ (56), 103 (85), 102 (44), 77 (79), 51 (62), 44 (82).

\section{Venturicidin A}

(100): $\quad \mathrm{C}_{41} \mathrm{H}_{67} \mathrm{NO}_{11}$ (750), farblose Kristalle, schwach UVlöschend (254 nm). $\boldsymbol{R}_{\boldsymbol{f}}=0.28 \quad\left(\mathrm{CH}_{2} \mathrm{Cl}_{2} /\right.$ $\mathrm{MeOH}$ 9:1). - Anfär-

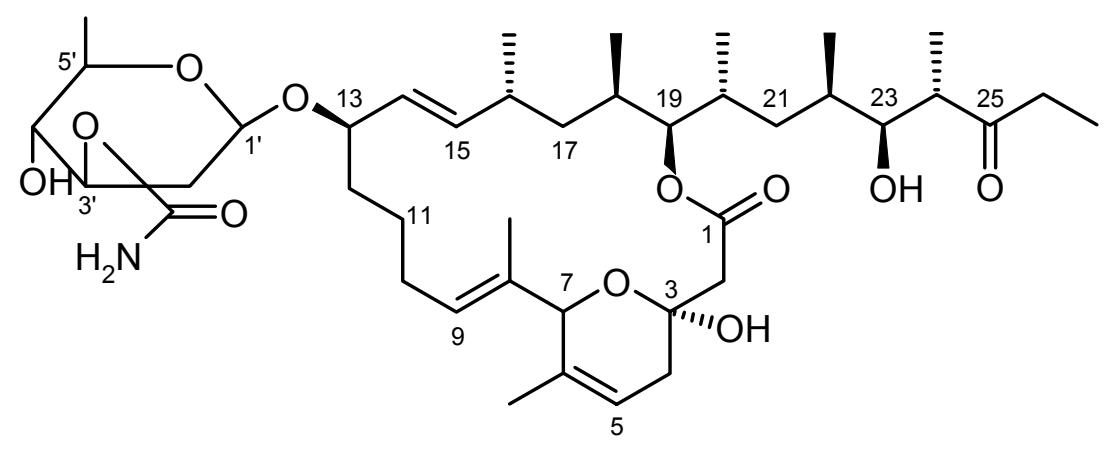
beverhalten: Anisaldehyd (dunkelblau), Ehrlich (dunkelblau). $-\boldsymbol{R}_{\boldsymbol{t}}=20.8 \mathrm{~min}$ (LC-MS). $-{ }^{\mathbf{1}} \mathbf{H}-\mathbf{N M R}\left(\mathrm{CDCl}_{3}, 300\right.$ $\mathrm{MHz}): \delta=5.58(\mathrm{~s}$ br, $1 \mathrm{H}, 3-\mathrm{OH}), 5.48(\mathrm{~m}, 1 \mathrm{H}, 5-\mathrm{H}), 5.39(\mathrm{~m}, 1 \mathrm{H}, 14-\mathrm{H}), 5.38(\mathrm{~m}, 1 \mathrm{H}, 9-\mathrm{H})$, 5.27 (m, 1H, 15-H), 4.82 (br, 2H, $\left.\mathrm{CONH}_{2}\right), 4.64$ (m, 2H, 19-CH, 3'-H), 4.54 (m, 1H, 1'-H), $4.44(\mathrm{~s}, 1 \mathrm{H}, 7-\mathrm{H}), 3.83(\mathrm{~m}, 1 \mathrm{H}, 13-\mathrm{H}), 3.51$ (m, 1H, 23-H), $3.23\left(\mathrm{~m}, 1 \mathrm{H}, 5^{\prime}-\mathrm{H}\right), 3.22$ (m, 1H, $\left.4^{\prime}-\mathrm{H}\right), 3.14$ (br, 1H, 4'-OH), $2.76(\mathrm{~m}, 1 \mathrm{H}, 24-\mathrm{H}), 2.59$ (m, 2H, 2-H), $2.57\left(\mathrm{~m}, 1 \mathrm{H}, 26-\mathrm{H}_{\mathrm{A}}\right)$, $2.53(\mathrm{~s} \mathrm{br}, 1 \mathrm{H}, 23-\mathrm{OH}), 2.30\left(\mathrm{~m}, 1 \mathrm{H}, 26-\mathrm{H}_{\mathrm{B}}\right), 2.24\left(\mathrm{~m}, 1 \mathrm{H}, 2^{\prime}-\mathrm{H}_{\mathrm{A}}\right), 2.14(\mathrm{~m}, 3 \mathrm{H}, 4-\mathrm{H}, 16-\mathrm{H})$, $2.09\left(\mathrm{~m}, 1 \mathrm{H}, 10-\mathrm{H}_{\mathrm{A}}\right), 1.90\left(\mathrm{~m}, 1 \mathrm{H}, 10-\mathrm{H}_{\mathrm{B}}\right), 1.83(\mathrm{~m}, 1 \mathrm{H}, 18-\mathrm{H}), 1.77(\mathrm{~m}, 1 \mathrm{H}, 20-\mathrm{H}), 1.67(\mathrm{~m}$, $\left.1 \mathrm{H}, 2^{\prime}-\mathrm{H}_{\mathrm{B}}\right), 1.60(\mathrm{~m}, 1 \mathrm{H}, 22-\mathrm{H}), 1.47\left(\mathrm{~s}, 3 \mathrm{H}, 6-\mathrm{CH}_{3}\right), 1.44(\mathrm{~m}, 2 \mathrm{H}, 12-\mathrm{H}), 1.44(\mathrm{~m}, 1 \mathrm{H}, 11-$ $\left.\mathrm{H}_{\mathrm{A}}\right), 1.39\left(\mathrm{~s}, 3 \mathrm{H}, 8-\mathrm{CH}_{3}\right), 1.31\left(\mathrm{~d}, 3 \mathrm{H}, 5^{\prime}-\mathrm{CH}_{3}\right), 1.20\left(\mathrm{~m}, 1 \mathrm{H}, 17-\mathrm{H}_{\mathrm{A}}\right), 1.17\left(\mathrm{~m}, 1 \mathrm{H}, 11-\mathrm{H}_{\mathrm{B}}\right)$, $1.15\left(\mathrm{~m}, 1 \mathrm{H}, 21-\mathrm{H}_{\mathrm{A}}\right), 1.08\left(\mathrm{~m}, 3 \mathrm{H}, 24-\mathrm{CH}_{3}\right), 1.03\left(\mathrm{~m}, 1 \mathrm{H}, 17-\mathrm{H}_{\mathrm{B}}\right), 1.03(\mathrm{~m}, 3 \mathrm{H}, 27-\mathrm{H}), 0.98$ $\left(\mathrm{m}, 1 \mathrm{H}, 21-\mathrm{H}_{\mathrm{B}}\right), 0.92\left(\mathrm{~d}, 3 \mathrm{H}, 16-\mathrm{CH}_{3}\right), 0.85\left(\mathrm{~m}, 3 \mathrm{H}, 22-\mathrm{CH}_{3}\right), 0.82\left(\mathrm{~m}, 3 \mathrm{H}, 20-\mathrm{CH}_{3}\right), 0.82(\mathrm{~m}$, $\left.3 \mathrm{H}, 18-\mathrm{CH}_{3}\right) .-{ }^{13} \mathbf{C}-\mathbf{N M R}\left(\mathrm{CDCl}_{3}, 150.8 \mathrm{MHz}\right): \delta=216.8\left(\mathrm{C}_{\mathrm{q}}, \mathrm{C}-25\right), 173.0\left(\mathrm{C}_{\mathrm{q}}, \mathrm{C}-1\right), 157.4$ $\left(\mathrm{C}_{\mathrm{q}}, \mathrm{C}-6^{\prime}\right), 138.8(\mathrm{CH}, \mathrm{C}-15), 134.4\left(\mathrm{C}_{\mathrm{q}}, \mathrm{C}-8\right), 133.1$ (C $\left.\mathrm{q}, \mathrm{C}-6\right), 129.5(\mathrm{CH}, \mathrm{C}-14), 129.4(\mathrm{CH}$, C-9), 116.7 (CH, C-5), 98.3 (CH, C-1'), 93.8 (C $\left.\mathrm{q}_{\mathrm{q}}, \mathrm{C}-3\right), 83.1$ (CH, C-19), 82.7 (CH, C-13), 
80.1 (CH, C-7), 77.8 (CH, C-23), $75.4\left(\mathrm{CH}, \mathrm{C}-3^{\prime}\right), 75.1\left(\mathrm{CH}, \mathrm{C}-4^{\prime}\right), 71.9$ (CH, C-5'), 48.3 (CH, C-24), $43.4\left(\mathrm{CH}_{2}, \mathrm{C}-2\right), 41.7\left(\mathrm{CH}_{2}, \mathrm{C}-21\right), 36.7\left(2 \mathrm{CH}_{2}, \mathrm{C}-17, \mathrm{C}-2^{\prime}\right), 36.0\left(\mathrm{CH}_{2}, \mathrm{C}-26\right)$, $35.1\left(\mathrm{CH}_{2}, \mathrm{C}-4\right), 34.80(\mathrm{CH}, \mathrm{C}-16), 34.78\left(\mathrm{CH}_{2}, \mathrm{C}-12\right), 32.4(\mathrm{CH}, \mathrm{C}-22), 32.1(\mathrm{CH}, \mathrm{C}-20)$, 31.6 (CH, C-18), $27.2\left(\mathrm{CH}_{2}, \mathrm{C}-10\right), 25.9\left(\mathrm{CH}_{2}, \mathrm{C}-11\right), 19.5\left(16-\mathrm{CH}_{3}\right), 19.2\left(6-\mathrm{CH}_{3}\right), 17.8\left(5^{\prime}-\right.$ $\left.\mathrm{CH}_{3}\right), 16.0\left(22-\mathrm{CH}_{3}\right), 14.3\left(24-\mathrm{CH}_{3}\right), 12.5\left(20-\mathrm{CH}_{3}\right), 12.0\left(18-\mathrm{CH}_{3}\right), 10.7\left(8-\mathrm{CH}_{3}\right), 7.5\left(\mathrm{CH}_{3}\right.$, C-27). - (+)-ESI-MS: $m / z(\%)=772[\mathrm{M}+\mathrm{Na}]^{+}(100) .-(-)-E S I-M S: ~ m / z(\%)=748[\mathrm{M}-\mathrm{H}]^{-}$ (60).

\section{$10.8 \quad$ Streptomyces sp. GW24/1811}

Der aus einer Bodenprobe aus Malta isolierte terrestrische Stamm bildete auf Agarplatten weiße Kolonien.

\subsubsection{Primärscreening}

Der nach dreitägiger Kultivierung erhaltene Rohextrakt von Stamm GW24/1811 wies im Dünnschichtchromatogramm eine Reihe farbloser Zonen auf. In einem zweiten, sechstätigen Kultivierungsversuch zeigte der erhaltene Rohextrakt im Dünnschichtchromatogramm mehrere orange und violette Zonen, welche sich bei Behandlung mit $2 \mathrm{~N} \mathrm{NaOH}$ blau bzw. braun verfärbten. Im Cytotoxizitätstest mit Artemia salina ergab sich eine Mortalitätsrate von 95 \%, während diese bei Caenorhabditis elegans $89 \%$ betrug.

Tabelle 12 Aktivität des Rohextraktes von Stamm GW24/1811 im biologischen Screening.

\begin{tabular}{|l|c|}
\hline \multicolumn{1}{|c|}{ Testkeim } & Hemmhof $\varnothing[\mathrm{mm}]$ \\
\hline \hline Bacillus subtilis & 14 \\
\hline Staphylococcus aureus & 11 \\
\hline Streptomyces viridochromogenes (Tü 57) & 11 \\
\hline Escherichia coli & 16 \\
\hline Candida albicans & 0 \\
\hline Mucor miehei (Tü 284) & 0 \\
\hline Chlorella vulgaris & 30 \\
\hline Chlorella sorokiniana & 0 \\
\hline Scenedesmus subspicatus & 0 \\
\hline
\end{tabular}




\subsubsection{Kultivierung und Aufarbeitung}

Die Fermentation erfolgte im 20 I Schüttelkultur-Maßstab in 1 I Erlenmeyerkolben, welche mit jeweils $200 \mathrm{ml} \mathrm{M}$-Medium befüllt waren und mit einem $1 \mathrm{~cm}^{2}$ großen Stück einer gut bewachsenen Agarplatte beimpft wurden. Die Kultivierung wurde auf einem Linearschüttler bei $28^{\circ} \mathrm{C}$ für 3 bzw. 6 Tage durchgeführt. Ab dem fünften Tag zeigten die Kulturen eine auffällige orange Färbung.

Die Kulturbrühe wurde anschließend mittels einer Filterpresse über Celite filtriert. Das erhaltene Filtrat wurde dreimal mit Essigester extrahiert, während das Mycel dreimal mit Essigester und zweimal mit Aceton unter Ultraschall-Behandlung extrahiert wurde. Die vereinigten organischen Phasen wurden vom Lösungsmittel befreit, wobei sich 6.4 g Rohextrakt ergaben.

\subsubsection{Isolierung und Charakterisierung der Metaboliten}

Der Rohextrakt wurde mittels Säulenchromatographie an Kieselgel mit einem Stufengradienten aus Chloroform/Methanol $\left(\mathrm{CHCl}_{3}, \mathrm{CHCl}_{3} / \mathrm{MeOH}\right.$ 99:1, $\mathrm{CHCl}_{3} / \mathrm{MeOH}$ 98:2, $\mathrm{CHCl}_{3} /$ $\mathrm{MeOH}$ 97:3, $\mathrm{CHCl}_{3} / \mathrm{MeOH}$ 96:4, $\mathrm{CHCl}_{3} / \mathrm{MeOH}$ 95:5, $\mathrm{CHCl}_{3} / \mathrm{MeOH}$ 9:1, $\mathrm{CHCl}_{3} / \mathrm{MeOH}$ 4:1 und $\mathrm{CHCl}_{3} / \mathrm{MeOH}$ 3:2) getrennt. Nach eintägiger Lagerung bei $4{ }^{\circ} \mathrm{C}$ im Lösungsmittel Chloroform fielen bei der ersten Fraktion 4.9 mg Hopen-B (101) in Form farbloser Kristalle an. Weitere chromatographische Aufreinigung der zweiten Fraktion an Sephadex LH-20 $\left(\mathrm{CH}_{2} \mathrm{Cl}_{2} /\right.$ $\mathrm{MeOH}$ 3:2) und anschließende präparative Dickschichtchromatographie lieferten $1.3 \mathrm{mg}$ Komodochinon A (103) und 4.6 mg Komodochinon B (104). Aus der dritten Fraktion konnten nach Chromatographie an Sephadex LH-20 $\left(\mathrm{CH}_{2} \mathrm{Cl}_{2} / \mathrm{MeOH} 3: 2\right) 56.0 \mathrm{mg}$ Poly(ßhydroxybuttersäure) (102) isoliert werden.

Hopen-B (101): $\mathrm{C}_{30} \mathrm{H}_{50}$ (410), farblose Kristalle, nicht UV-aktiv. $-\boldsymbol{R}_{\boldsymbol{f}}=0.98\left(\mathrm{CH}_{2} \mathrm{Cl}_{2} / \mathrm{MeOH} 95: 5\right)$. Anfärbeverhalten: Anisaldehyd (violett). $-\boldsymbol{R}_{\boldsymbol{t}}=12.2$ $\min (\mathrm{LC}-\mathrm{MS}) .-{ }^{\mathbf{1}} \mathbf{H}-\mathbf{N M R}\left(\mathrm{CDCl}_{3}, 300 \mathrm{MHz}\right): \delta=$ 4.76 (s, 2H, 23-H), 2.66 (q, 1H, 21-H), 1.83 (m, 2H, 20-H), 1.73 (s, 3H, 24-H), $1.62(\mathrm{~m}, 2 \mathrm{H}, 1-\mathrm{H}), 1.60$

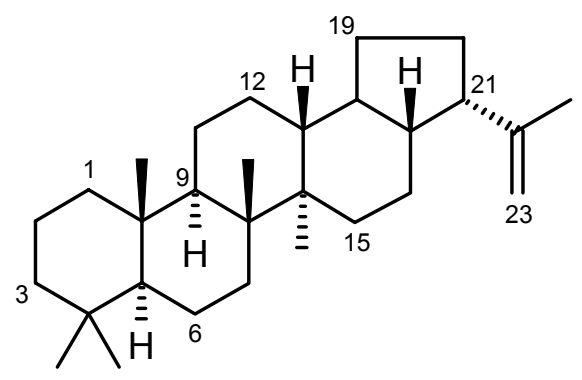
(m, 2H, 16-H), 1.47 (m, 2H, 6-H), 1.35 (m, 2H, 2-H), 1.31 (m, 2H, 3-H), 1.20 (m, 2H, 15-H), 0.94 (s, 3H, 28-H), 0.92 (s, 3H, 29-H), 0.82 (s, 3H, 25-H), 0.79 (s, 3H, 27-H), 0.77 (s, 3H, 26-H), 0.70 (s, 3H, 30-H), 0.68 (m, 1H, 5-H). $-{ }^{13} \mathbf{C}-$ 
NMR $\left(\mathrm{CDCl}_{3}, 125.7 \mathrm{MHz}\right): \delta=148.8\left(\mathrm{C}_{\mathrm{q}}, \mathrm{C}-22\right), 110.1\left(\mathrm{CH}_{2}, \mathrm{C}-23\right), 56.1(\mathrm{CH}, \mathrm{C}-5), 54.9$ (CH, C-17), 50.4 (CH, C-9), $49.4(\mathrm{CH}, \mathrm{C}-13), 46.5$ (CH, C-21), $44.8\left(\mathrm{C}_{\mathrm{q}}, \mathrm{C}-18\right), 42.10\left(\mathrm{CH}_{2}\right.$, C-3), $42.05\left(\mathrm{C}_{\mathrm{q}}, \mathrm{C}-14\right), 41.89\left(\mathrm{C}_{\mathrm{q}}, \mathrm{C}-8\right), 41.88\left(\mathrm{CH}_{2}, \mathrm{C}-19\right), 40.3\left(\mathrm{CH}_{2}, \mathrm{C}-1\right), 37.4\left(\mathrm{C}_{\mathrm{q}}, \mathrm{C}-10\right)$, $33.6\left(\mathrm{CH}_{2}, \mathrm{C}-15\right), 33.4\left(\mathrm{CH}_{3}, \mathrm{C}-25\right), 33.2\left(\mathrm{C}_{\mathrm{q}}, \mathrm{C}-4\right), 33.2\left(\mathrm{CH}_{2}, \mathrm{C}-7\right), 27.4\left(\mathrm{CH}_{2}, \mathrm{C}-20\right), 25.0$ $\left(\mathrm{CH}_{3}, \mathrm{C}-26\right), 24.0\left(\mathrm{CH}_{2}, \mathrm{C}-12\right), 21.7\left(\mathrm{CH}_{2}, \mathrm{C}-16\right), 21.6\left(\mathrm{CH}_{3}, \mathrm{C}-24\right), 20.9\left(\mathrm{CH}_{2}, \mathrm{C}-11\right), 18.7$ $\left(2 \mathrm{CH}_{2}, \mathrm{C}-2, \mathrm{C}-6\right), 16.74\left(\mathrm{CH}_{3}, \mathrm{C}-29\right), 16.68\left(\mathrm{CH}_{3}, \mathrm{C}-28\right), 16.1\left(\mathrm{CH}_{3}, \mathrm{C}-30\right), 15.8\left(\mathrm{CH}_{3}, \mathrm{C}-27\right)$. - EI-MS (70 eV): $m / z(\%)=410\left(\left([\mathrm{M}]^{\circ+}, 13\right), 191\right.$ (68), 189 (38), 137 (34), 123 (54), 111 (47), 109 (70), 97 (52), 95 (98), 85 (39), 83 (79), 81 (89), 71 (63), 69 (100), 67 (45), 43 (58), $41(41)$.

Poly(ß-hydroxybuttersäure) (102): weißer Feststoff, nicht UVaktiv. - Keine Anfärbung. - ${ }^{\mathbf{1}} \mathbf{H}-\mathbf{N M R}\left(\mathrm{CDCl}_{3}, 300 \mathrm{MHz}\right): \delta=$ $5.23\left(\mathrm{sext},{ }^{3} J=6.0 \mathrm{~Hz}, \mathrm{CH}\right), 2.59\left(\mathrm{dd},{ }^{2} J=15.5 \mathrm{~Hz},{ }^{3} J=7.6 \mathrm{~Hz}\right.$,

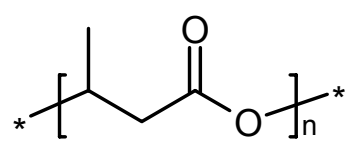
$\left.\mathrm{CH}_{2}, \mathrm{H}_{\mathrm{A}}\right), 2.44\left(\mathrm{dd},{ }^{2} \mathrm{~J}=15.5 \mathrm{~Hz},{ }^{3} \mathrm{~J}=5.8 \mathrm{~Hz}, \mathrm{CH}_{2}, \mathrm{H}_{\mathrm{B}}\right), 1.24(\mathrm{~d}$, $\left.{ }^{3} J=6.0 \mathrm{~Hz}, \mathrm{CH}_{3}\right) .-{ }^{13} \mathbf{C}-\mathrm{NMR}\left(\mathrm{CDCl}_{3}, 150.8 \mathrm{MHz}\right): \delta=169.1(\mathrm{COO}), 67.6(\mathrm{CH}), 40.8$ $\left(\mathrm{CH}_{2}\right), 19.8\left(\mathrm{CH}_{3}\right)$.

Komodochinon A (103): $\mathrm{C}_{27} \mathrm{H}_{31} \mathrm{NO}_{10}$ (529), orange Feststoff, UV-löschend (254 $\mathrm{nm}) .-\boldsymbol{R}_{\boldsymbol{f}}=0.42\left(\mathrm{CH}_{2} \mathrm{Cl}_{2} / \mathrm{MeOH} 95: 5\right) .-$ Keine Anfärbung. - $\boldsymbol{R}_{\boldsymbol{t}}=12.4 \mathrm{~min}$ (LCMS). - ${ }^{1}$ H-NMR ([D $]$ DMSO, $\left.600 \mathrm{MHz}\right)$ : $\delta=8.40$ (s br, 2H, 2OH) 7.95 (m, 1H, 1H), 7.84 (m, 1H, 2-H), 7.74 (m, 1H, 3-H), 5.78 (br s, 1H, 1'-H), 4.97 (m, 1H, 7-H),

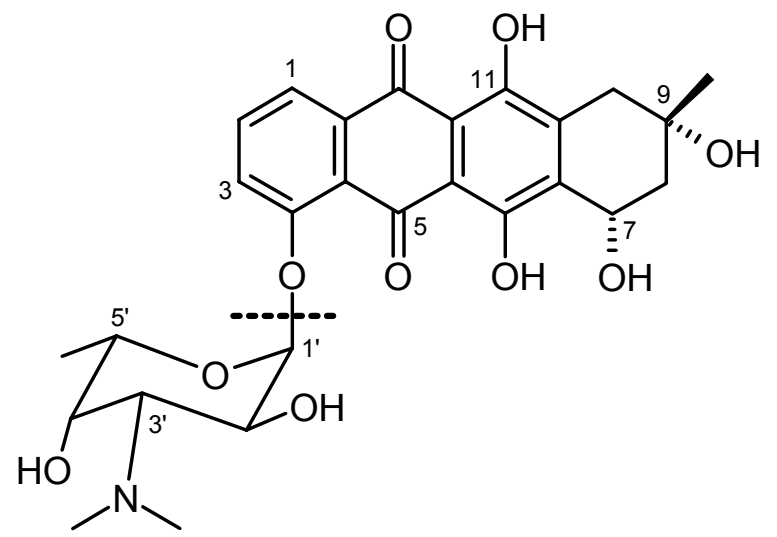
4.18 (d br, 1H, 2'-H), 3.98 (q, ${ }^{3} J=6.1 \mathrm{~Hz}$, $\left.1 \mathrm{H}, 5^{\prime}-\mathrm{H}\right), 3.83$ (d br, 1H, 3'-H), 3.23 (t br, 1H, 4'-H), 2.97 (d, $\left.{ }^{2} J=18.7 \mathrm{~Hz}, 1 \mathrm{H}, 10-\mathrm{H}_{\mathrm{A}}\right), 2.57$ $\left(\mathrm{d},{ }^{2} J=18.7 \mathrm{~Hz}, 1 \mathrm{H}, 10-\mathrm{H}_{\mathrm{B}}\right), 2.57\left(\mathrm{~s}, 3 \mathrm{H}, \mathrm{N}-\mathrm{CH}_{3}\right), 2.58\left(\mathrm{~s}, 3 \mathrm{H}, \mathrm{N}-\mathrm{CH}_{3}\right), 2.02\left(\mathrm{~d},{ }^{2} J=13.7 \mathrm{~Hz}\right.$, 1H, 8- $\left.\mathrm{H}_{\mathrm{A}}\right), 1.83\left(\mathrm{~m}, 1 \mathrm{H}, 8-\mathrm{H}_{\mathrm{B}}\right), 1.28(\mathrm{~s}, 3 \mathrm{H}, 13-\mathrm{H}), 1.04\left(\mathrm{~d},{ }^{3} \mathrm{~J}=6.1 \mathrm{~Hz}, 3 \mathrm{H}, 6\right.$ '-H). - (+)-ESIMS: $m / z(\%)=1609[3 \mathrm{M}+\mathrm{Na}]^{+}(44), 1081[2 \mathrm{M}+\mathrm{Na}]^{+}(100), 1059[2 \mathrm{M}+\mathrm{H}]^{+}(82), 530[\mathrm{M}+\mathrm{H}]^{+}$ (38). - (-)-ESI-MS: $m / z(\%)=1080\left[2 \mathrm{M}+\mathrm{Na}-2 \mathrm{H}^{-}(100), 529[\mathrm{M}-\mathrm{H}]^{-}(72)\right.$. 


\section{Komodochinon B (104):}

$\mathrm{C}_{19} \mathrm{H}_{16} \mathrm{O}_{7}$ (356), roter Feststoff, UV-löschend (254 nm) und orange fluoreszierend (366 nm). $-\boldsymbol{R}_{\boldsymbol{f}}=0.42$ $\left(\mathrm{CH}_{2} \mathrm{Cl}_{2} / \mathrm{MeOH} 95: 5\right)$. - Keine Anfärbung. $-\boldsymbol{R}_{\boldsymbol{t}}=19.6$ $\min$ (LC-MS). $-{ }^{\mathbf{1}} \mathbf{H}-\mathbf{N M R}\left(\left[\mathrm{D}_{6}\right] \mathrm{DMSO}, 300 \mathrm{MHz}\right): \delta$ $=13.40(\mathrm{~s} \mathrm{br}, 1 \mathrm{H}, 11-\mathrm{OH}), 12.06(\mathrm{~s} \mathrm{br}, 1 \mathrm{H}, 4-\mathrm{OH})$,<smiles>C[C@]1(O)Cc2c(c(O)c3[14c](c2O)C(=O)c2cccc(O)c2C3=O)[C@@H](O)C1</smiles>
$7.81\left(\mathrm{dd},{ }^{3} J=7.7 \mathrm{~Hz}, 7.5 \mathrm{~Hz}, 1 \mathrm{H}, 2-\mathrm{H}\right), 7.75\left(\mathrm{dd},{ }^{3} J=7.5 \mathrm{~Hz},{ }^{4} J=1.5 \mathrm{~Hz}, 1 \mathrm{H}, 1-\mathrm{H}\right), 7.37$ (dd, $\left.{ }^{3} J=7.7 \mathrm{~Hz},{ }^{4} J=1.5 \mathrm{~Hz}, 1 \mathrm{H}, 3-\mathrm{H}\right), 5.28$ (s, 1H, OH), 4.94 (s br, 1H, 7-H), 3.02 (d, ${ }^{2} J=18.5$ $\left.\mathrm{Hz}, 1 \mathrm{H}, 10-\mathrm{H}_{\mathrm{A}}\right), 2.53\left(\mathrm{~d},{ }^{2} J=18.5 \mathrm{~Hz}, 1 \mathrm{H}, 10-\mathrm{H}_{\mathrm{B}}\right), 2.05\left(\mathrm{dd},{ }^{2} J=13.7 \mathrm{~Hz}, 1 \mathrm{H}, 8-\mathrm{H}_{\mathrm{A}}\right), 1.84$ $\left(\mathrm{dd},{ }^{2} J=13.7 \mathrm{~Hz}, 1 \mathrm{H}, 8-\mathrm{H}_{\mathrm{B}}\right), 1.32(\mathrm{~s}, 3 \mathrm{H}, 13-\mathrm{H}) .-{ }^{13} \mathbf{C}-\mathbf{N M R}\left(\left[\mathrm{D}_{6}\right] \mathrm{DMSO}, 150.8 \mathrm{MHz}\right): \delta=$ 189.7 ( $\left.\mathrm{C}_{\mathrm{q}}, \mathrm{C}-5\right), 185.7$ ( $\left.\mathrm{C}_{\mathrm{q}}, \mathrm{C}-12\right), 161.4\left(\mathrm{C}_{\mathrm{q}}, \mathrm{C}-4\right), 156.4\left(\mathrm{C}_{\mathrm{q}}, \mathrm{C}-6\right), 156.1\left(\mathrm{C}_{\mathrm{q}}, \mathrm{C}-11\right), 137.9$ $\left(\mathrm{C}_{\mathrm{q}}, \mathrm{C}-6 \mathrm{a}\right), 137.1$ (CH, C-2), $137.0\left(\mathrm{C}_{\mathrm{q}}, \mathrm{C}-10 \mathrm{a}\right), 133.0\left(\mathrm{C}_{\mathrm{q}}, \mathrm{C}-12 \mathrm{a}\right), 124.4(\mathrm{CH}, \mathrm{C}-3), 118.9$ $(\mathrm{CH}, \mathrm{C}-1), 115.9\left(\mathrm{C}_{\mathrm{q}}, \mathrm{C}-4 \mathrm{a}\right), 110.3\left(\mathrm{C}_{\mathrm{q}}, \mathrm{C}-5 \mathrm{a}\right), 109.6\left(\mathrm{C}_{\mathrm{q}}, \mathrm{C}-11 \mathrm{a}\right), 67.5\left(\mathrm{C}_{\mathrm{q}}, \mathrm{C}-9\right), 61.4(\mathrm{CH}, \mathrm{C}-$ 7), $40.4\left(\mathrm{CH}_{2}, \mathrm{C}-8\right), 37.4\left(\mathrm{CH}_{2}, \mathrm{C}-10\right), 29.8\left(\mathrm{CH}_{3}, \mathrm{C}-13\right)$. - (+)-ESI-MS: $m / z(\%)=1091$ $[3 \mathrm{M}+\mathrm{Na}]^{+}(45), 735[2 \mathrm{M}+\mathrm{Na}]^{+}(100), 379[\mathrm{M}+\mathrm{Na}]^{+}(7) .-(-)-\mathbf{E S I}-\mathbf{M S}: m / z(\%)=733$ $[2 \mathrm{M}+\mathrm{Na}-2 \mathrm{H}]^{+}(100), 355[\mathrm{M}-\mathrm{H}]^{-}(52)$.

\section{$10.9 \quad$ Streptomyces sp. GW16/3128}

Sowohl Agarplatten also auch Mycel und Kulturbrühe vom terrestrischen Stamm Streptomyces sp. GW16/3128 waren tief schwarz gefärbt.

\subsubsection{Primärscreening}

Im Dünnschichtchromatogramm des hellgelben Rohextraktes fiel eine Zone auf, die schwach UV-löschend war und sich mit Anisaldehyd schwarz anfärbte. 
Tabelle 13 Aktivität des Rohextraktes von Stamm GW16/3128 im biologischen Screening.

\begin{tabular}{|l|c|}
\hline \multicolumn{1}{|c|}{ Testkeim } & Hemmhof $\varnothing[\mathrm{mm}]$ \\
\hline \hline Bacillus subtilis & 21 \\
\hline Staphylococcus aureus & 18 \\
\hline Streptomyces viridochromogenes (Tü 57) & 20 \\
\hline Escherichia coli & 20 \\
\hline Candida albicans & 0 \\
\hline Mucor miehei (Tü 284) & 0 \\
\hline Chlorella vulgaris & sehr hoch \\
\hline Chlorella sorokiniana & 40 \\
\hline Scenedesmus subspicatus & 32 \\
\hline
\end{tabular}

\subsubsection{Kultivierung und Aufarbeitung}

Die Kultivierung von GW16/3128 erfolgte im 201 Schüttelkultur-Maßstab in 11 Erlenmeyerkolben, welche mit je $200 \mathrm{ml}$ Fleischextrakt-Medium befüllt waren und auf einem Linearschüttler drei Tage bei $28^{\circ} \mathrm{C}$ inkubiert wurden. Zum Beimpfen diente eine sechs Tage alte Vorkultur, welche im selben Medium angezüchtet wurde.

Die Aufarbeitung der Kulturbrühe erfolgte wie in Kapitel 10.8.2 beschrieben, wobei sich $3.3 \mathrm{~g}$ Rohextrakt ergaben.

\subsubsection{Isolierung und Charakterisierung der Metaboliten}

Der Rohextrakt wurde mittels Säulenchromatographie an Kieselgel mit einem Stufengradienten aus Chloroform/Methanol $\left(\mathrm{CHCl}_{3}, \mathrm{CHCl}_{3} / \mathrm{MeOH}\right.$ 99:1, $\mathrm{CHCl}_{3} / \mathrm{MeOH}$ 98:2, $\mathrm{CHCl}_{3} /$ $\mathrm{MeOH}$ 97:3, $\mathrm{CHCl}_{3} / \mathrm{MeOH}$ 96:4, $\mathrm{CHCl}_{3} / \mathrm{MeOH}$ 95:5, $\mathrm{CHCl}_{3} / \mathrm{MeOH}$ 9:1, $\mathrm{CHCl}_{3} / \mathrm{MeOH}$ 4:1 und $500 \mathrm{ml} \mathrm{CHCl}_{3} / \mathrm{MeOH}$ 3:2) getrennt. Anschließende Trennung an Sephadex LH-20 $\left(\mathrm{CHCl}_{3} / \mathrm{MeOH}\right.$ 3:2 und $\left.\mathrm{MeOH}\right)$ und Säulenchromatographie an Kieselgel führte $\mathrm{zu} 6.4 \mathrm{mg}$ Glykolipid 105. 


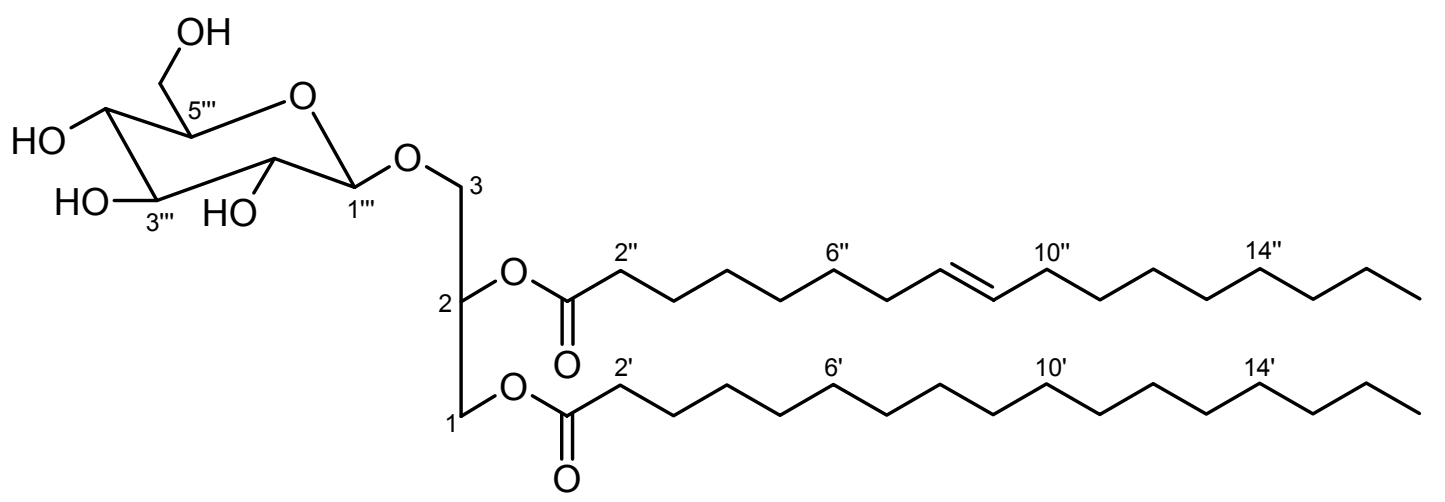

Glykolipid 105: $\mathrm{C}_{43} \mathrm{H}_{80} \mathrm{O}_{10}$ (756), weißer Feststoff, schwach UV-löschend (254 nm). - $\boldsymbol{R}_{\boldsymbol{f}}=$ $0.20\left(\mathrm{CH}_{2} \mathrm{Cl}_{2} / \mathrm{MeOH}\right.$ 95:5). - Anfärbeverhalten: Anisaldehyd (schwarz). - ${ }^{\mathbf{1}} \mathbf{H}-\mathbf{N M R}$ $\left(\mathrm{CD}_{3} \mathrm{OD}, 300 \mathrm{MHz}\right): \delta=5.33\left(\mathrm{t}, 2 \mathrm{H},{ }^{3} J=12.0 \mathrm{~Hz}\right), 5.24(\mathrm{~m}, 1 \mathrm{H}, 2-\mathrm{H}), 4.79\left(\mathrm{~d},{ }^{3} J=8.3 \mathrm{~Hz}\right.$, $1 \mathrm{H}, 1$ ', $-\mathrm{H}), 4.47\left(\mathrm{dd},{ }^{2} J=12.0 \mathrm{~Hz},{ }^{3} J=3.2 \mathrm{~Hz}, 1 \mathrm{H}, 1-\mathrm{H}_{\mathrm{A}}\right), 4.20\left(\mathrm{dd},{ }^{2} J=12.0 \mathrm{~Hz},{ }^{3} J=6.4\right.$ $\left.\mathrm{Hz}, 1 \mathrm{H}, 1-\mathrm{H}_{\mathrm{B}}\right), 3.86\left(\mathrm{dd},{ }^{2} J=11.2 \mathrm{~Hz},{ }^{3} J=6.4 \mathrm{~Hz}, 1 \mathrm{H}, 3-\mathrm{H}_{\mathrm{A}}\right), 3.78\left(\mathrm{dd},{ }^{2} J=10.5 \mathrm{~Hz},{ }^{3} J=2.3\right.$ $\mathrm{Hz}, 1 \mathrm{H}, 6$, $\left.{ }^{\prime}-\mathrm{H}_{\mathrm{A}}\right), 3.66\left(\mathrm{dd},{ }^{2} J=10.5 \mathrm{~Hz},{ }^{3} J=6.3 \mathrm{~Hz}, 1 \mathrm{H}, 6{ }^{\prime \prime}{ }^{-}-\mathrm{H}_{\mathrm{B}}\right), 3.62\left(\mathrm{~m}, 1 \mathrm{H}, 3-\mathrm{H}_{\mathrm{B}}\right), 3.60$

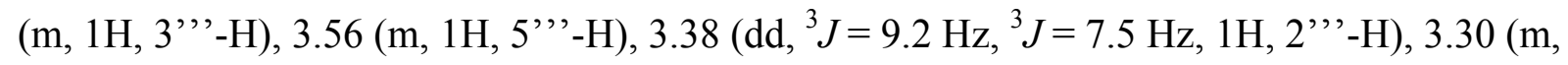
1H, 4','-H), 2.32 (m, 4H, 2'-H, 2' '-H), 2.02 (m, 4H), 1.60 (m, 4H, 3'-H, 3'”-H), 1.31 (br, 44H, 22 Alkyl-CH $), 0.90$ (m, 6H, 17'-H, 17' -H). - ${ }^{13} \mathbf{C}-\mathbf{N M R}\left(\mathrm{CD}_{3} \mathrm{OD}, 150.8 \mathrm{MHz}\right): \delta=175.0$ (C $\mathrm{C}_{\mathrm{q}}, 1$ '-COO), 174.7 (C $\mathrm{q}, 1$ '’-COO), $130.9(\mathrm{CH}), 130.8(\mathrm{CH}), 100.7(\mathrm{CH}, \mathrm{C}-1$ ', '), $74.9(\mathrm{CH}$, C-3",'), 73.9 (CH, C-5','), 73.4 (CH, C-2'”'), 71.62 (CH, C-2), 71.57 (CH, C-4"’'), 67.1 $\left(\mathrm{CH}_{2}, \mathrm{C}-3\right), 63.8\left(\mathrm{CH}_{2}, \mathrm{C}-1\right), 62.6\left(\mathrm{CH}_{2}, \mathrm{C}-6\right.$ '’' $), 35.2\left(\mathrm{CH}_{2}, \mathrm{C}-2\right.$ '), $35.0\left(\mathrm{CH}_{2}, \mathrm{C}-2\right.$ '’), 33.1 $\left(\mathrm{CH}_{2}\right), 33.0\left(\mathrm{CH}_{2}\right), 30.9\left(9 \mathrm{CH}_{2}\right), 28.20\left(\mathrm{CH}_{2}\right), 28.17\left(\mathrm{CH}_{2}\right), 26.1\left(\mathrm{CH}_{2}, \mathrm{C}-3\right), 26.0\left(\mathrm{CH}_{2}, \mathrm{C}-\right.$ 3'’), $23.8\left(2 \mathrm{CH}_{2}, \mathrm{C}-16\right.$ ', C-16''), $14.6\left(\mathrm{CH}_{3}, \mathrm{C}-17\right.$ ', C-17'').

\subsection{Streptomyces sp. GW12/1492}

\subsubsection{Primärscreening}

Der Rohextrakt zeigte im Dünnschichtchromatogramm zwei auffällige Zonen. Die unpolarere Zone besaß eine gelbe Eigenfarbe und färbte sich mit herkömmlichen Sprühreagenzien nicht an, während die polarere Zone farblos war, beim Besprühen mit Anisaldehyd und Ehrlichs Reagenz jedoch eine dunkelblaue Farbreaktion einging. 
Tabelle 14 Aktivität des Rohextraktes von Stamm GW12/1492 im biologischen Screening.

\begin{tabular}{|l|c|}
\hline \multicolumn{1}{|c|}{ Testkeim } & Hemmhof $\varnothing[\mathrm{mm}]$ \\
\hline Bacillus subtilis & 11 \\
\hline Staphylococcus aureus & 13 \\
\hline Streptomyces viridochromogenes (Tü 57) & 20 \\
\hline Escherichia coli & 0 \\
\hline Candida albicans & 0 \\
\hline Mucor miehei (Tü 284) & 0 \\
\hline Chlorella vulgaris & 0 \\
\hline Chlorella sorokiniana & 0 \\
\hline Scenedesmus subspicatus & 0 \\
\hline
\end{tabular}

\subsubsection{Kultivierung und Aufarbeitung}

Die Kultivierung erfolgte im 20 I Schüttelkultur-Maßstab in 1 I Erlenmeyerkolben, welche mit je $200 \mathrm{ml} \mathrm{M}$-Medium befüllt waren und mit einem $1 \mathrm{~cm}^{2}$ großen Stück einer gut bewachsenen Agarplatte beimpft wurden. Die Fermentation wurde auf einem Linearschüttler bei $28^{\circ} \mathrm{C}$ für vier Tage durchgeführt, wobei sich ab dem zweiten Tag eine Gelbfärbung der Kulturbrühe einstellte.

Die Aufarbeitung der Kulturen erfolgte wie in Kapitel 10.8.2 beschrieben. Es ergaben sich so $15.2 \mathrm{~g}$ Rohextrakt.

\subsubsection{Isolierung und Charakterisierung der Metaboliten}

Der Rohextrakt wurde mittels Säulenchromatographie an Kieselgel mit einem Stufengradienten aus Chloroform/Methanol $\left(\mathrm{CHCl}_{3}, \mathrm{CHCl}_{3} / \mathrm{MeOH}\right.$ 99:1, $\mathrm{CHCl}_{3} / \mathrm{MeOH}$ 98:2, $\mathrm{CHCl}_{3} /$ $\mathrm{MeOH}$ 97:3, $\mathrm{CHCl}_{3} / \mathrm{MeOH}$ 96:4, $\mathrm{CHCl}_{3} / \mathrm{MeOH}$ 95:5, $\mathrm{CHCl}_{3} / \mathrm{MeOH}$ 9:1, $\mathrm{CHCl}_{3} / \mathrm{MeOH}$ 4:1 und $\mathrm{CHCl}_{3} / \mathrm{MeOH}$ 3:2) getrennt. Nach mehreren Trennversuchen an Kieselgel gelang die Isolierung von $4.7 \mathrm{mg}$ Cervinomycin $\mathrm{A}_{2}$ (107). Aus der zweiten Fraktion konnten durch Säulenchromatographie an Kieselgel 2 g Hexa- und Heptadecansäure (108) erhalten werden. Die weitere Auftrennung der dritten Fraktion an Sephadex LH-20 $\left(\mathrm{CH}_{2} \mathrm{Cl}_{2} / \mathrm{MeOH}\right)$ lieferte $2.2 \mathrm{~g} \mathrm{X}$-14952B (106), während aus Fraktion vier $2.0 \mathrm{mg}$ Anthranilsäureamid und $4.0 \mathrm{mg}$ Phenylessigsäure isoliert werden konnten. 


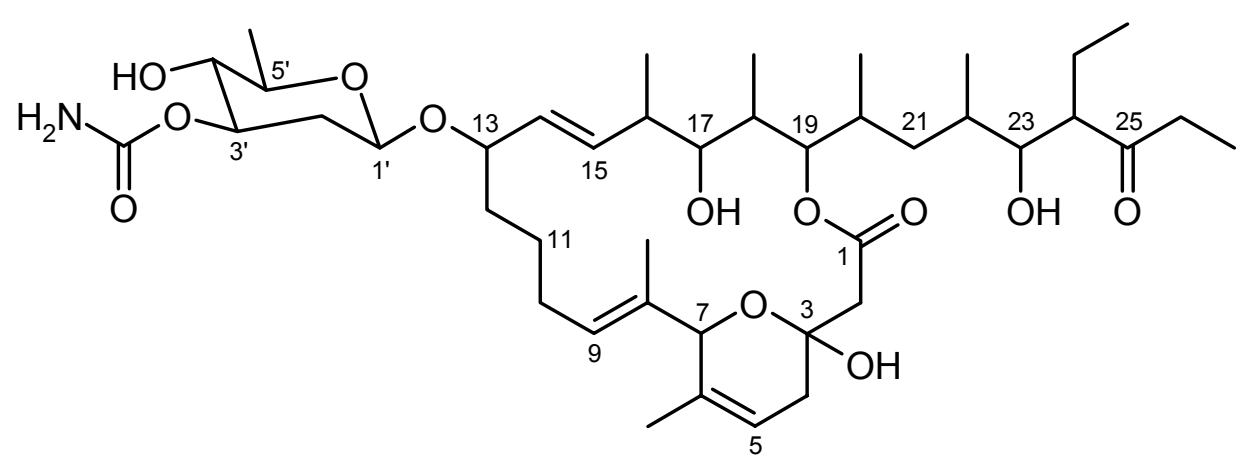

X-14952B (106): $\mathrm{C}_{42} \mathrm{H}_{69} \mathrm{NO}_{12}$ (779), weißer Feststoff, schwach UV-löschend (254 nm). - $\boldsymbol{R}_{\boldsymbol{f}}$ $=0.28\left(\mathrm{CH}_{2} \mathrm{Cl}_{2} / \mathrm{MeOH}\right.$ 95:5). - Anfärbeverhalten: Anisaldehyd (dunkelblau), Ehrlich (dunkelblau). $-\boldsymbol{R}_{\boldsymbol{t}}=20.1 \mathrm{~min}$ (LC-MS). - ${ }^{1} \mathbf{H}-\mathbf{N M R}\left(\mathrm{CDCl}_{3}, 300 \mathrm{MHz}\right): \delta=5.61$ (s br, $1 \mathrm{H}$, $\mathrm{OH}), 5.51\left(\mathrm{dd},{ }^{3} J=15.4 \mathrm{~Hz}, 8.6 \mathrm{~Hz}, 1 \mathrm{H}, 15-\mathrm{H}\right), 5.44(\mathrm{~m}, 1 \mathrm{H}, 5-\mathrm{H}), 5.40$ (dd, ${ }^{3} J=10.3 \mathrm{~Hz}, 5.1$ $\mathrm{Hz}, 1 \mathrm{H}, 9-\mathrm{H}), 5.19$ (dd, $\left.{ }^{3} J=15.4 \mathrm{~Hz}, 9.6 \mathrm{~Hz}, 1 \mathrm{H}, 14-\mathrm{H}\right), 5.03$ (s br, 2H, $\mathrm{NH}_{2}$ ), 4.82 (dd, ${ }^{3} J=$ $8.9 \mathrm{~Hz}, 1.9 \mathrm{~Hz}, 1 \mathrm{H}, 19-\mathrm{H}), 4.59$ (m, 1H, 3'-H), 4.52 (dd, ${ }^{3} J=9.8 \mathrm{~Hz}, 1.9 \mathrm{~Hz}, 1 \mathrm{H}, 1^{\prime}$-H), 4.41 (s, 1H, 7-H), 3.89 (m, 1H, 13-H), 3.50 (m, 1H, 23-H), 3.27 (m, 1H, 17-H), 3.23 (m, 1H, 5'H), $3.18\left(\mathrm{t},{ }^{3} J=8.9 \mathrm{~Hz}, 1 \mathrm{H}, 4^{\prime}-\mathrm{H}\right), 2.64(\mathrm{~m}, 1 \mathrm{H}, 24-\mathrm{H}), 2.62\left(\mathrm{~d},{ }^{2} J=16.7 \mathrm{~Hz}, 1 \mathrm{H}, 2-\mathrm{H}_{\mathrm{A}}\right), 2.54$ $\left(\mathrm{d},{ }^{2} J=16.7 \mathrm{~Hz}, 1 \mathrm{H}, 2-\mathrm{H}_{\mathrm{B}}\right), 2.47$ (q, $\left.{ }^{3} J=7.3 \mathrm{~Hz}, 2 \mathrm{H}, 26-\mathrm{H}\right), 2.22\left(\mathrm{ddd},{ }^{2} J=12.3 \mathrm{~Hz},{ }^{3} J=5.2\right.$ Hz, 1.8 Hz, 1H, 2'- $\left.\mathrm{H}_{\mathrm{A}}\right), 2.12(\mathrm{~m}, 1 \mathrm{H}, 16-\mathrm{H}), 2.08$ (m, 2H, 4-H), $2.06\left(\mathrm{~m}, 1 \mathrm{H}, 10-\mathrm{H}_{\mathrm{A}}\right), 1.95$ (m, $1 \mathrm{H}, 18-\mathrm{H}), 1.83\left(\mathrm{~m}, 1 \mathrm{H}, 10-\mathrm{H}_{\mathrm{B}}\right), 1.74(\mathrm{~m}, 1 \mathrm{H}, 22-\mathrm{H}), 1.64\left(\mathrm{~m}, 1 \mathrm{H}, 2^{\prime}-\mathrm{H}_{\mathrm{B}}\right), 1.56(\mathrm{~m}, 3 \mathrm{H}, 12-$ $\left.\mathrm{H}_{\mathrm{B}}, 24-\mathrm{CH}_{2} \mathrm{CH}_{3}\right), 1.55(\mathrm{~m}, 1 \mathrm{H}, 20-\mathrm{H}), 1.44\left(\mathrm{~s}, 3 \mathrm{H}, 6-\mathrm{CH}_{3}\right), 1.41\left(\mathrm{~m}, 2 \mathrm{H}, 11-\mathrm{H}_{\mathrm{A}}\right), 1.40(\mathrm{~m}, 1 \mathrm{H}$, $\left.12-\mathrm{H}_{\mathrm{B}}\right), 1.36\left(\mathrm{~s}, 3 \mathrm{H}, 8-\mathrm{CH}_{3}\right), 1.28\left(\mathrm{~d},{ }^{3} \mathrm{~J}=6.0 \mathrm{~Hz}, 3 \mathrm{H}, 5^{\prime}-\mathrm{CH}_{3}\right), 1.20\left(\mathrm{~m}, 1 \mathrm{H}, 11-\mathrm{H}_{\mathrm{B}}\right), 1.19$ (m, $\left.1 \mathrm{H}, 21-\mathrm{H}_{\mathrm{A}}\right), 1.00\left(\mathrm{t},{ }^{3} \mathrm{~J}=7.1 \mathrm{~Hz}, 3 \mathrm{H}, 27-\mathrm{H}\right), 0.94\left(\mathrm{~m}, 1 \mathrm{H}, 21-\mathrm{H}_{\mathrm{B}}\right), 0.90\left(\mathrm{~d},{ }^{3} J=6.7 \mathrm{~Hz}, 3 \mathrm{H}\right.$, $\left.16-\mathrm{CH}_{3}\right), 0.86\left(\mathrm{~d},{ }^{3} \mathrm{~J}=7.0 \mathrm{~Hz}, 3 \mathrm{H}, 18-\mathrm{CH}_{3}\right), 0.84\left(\mathrm{t},{ }^{3} \mathrm{~J}=7.4 \mathrm{~Hz}, 3 \mathrm{H}, 24-\mathrm{CH}_{2} \mathrm{CH}_{3}\right), 0.82\left(\mathrm{~d},{ }^{3} \mathrm{~J}\right.$ $\left.=6.7 \mathrm{~Hz}, 3 \mathrm{H}, 20-\mathrm{CH}_{3}\right), 0.80\left(\mathrm{~d},{ }^{3} \mathrm{~J}=6.7 \mathrm{~Hz}, 3 \mathrm{H}, 22-\mathrm{CH}_{3}\right) .-{ }^{13} \mathbf{C}-\mathbf{N M R}\left(\mathrm{CDCl}_{3}, 150.8 \mathrm{MHz}\right)$ : $\delta=217.2\left(\mathrm{C}_{\mathrm{q}}, \mathrm{C}-25\right), 173.5\left(\mathrm{C}_{\mathrm{q}}, \mathrm{C}-1\right), 157.4\left(\mathrm{C}_{\mathrm{q}}, 3^{\prime}-\mathrm{OCONH}_{2}\right), 134.8\left(\mathrm{C}_{\mathrm{q}}, \mathrm{C}-8\right), 134.3(\mathrm{CH}$, C-14), 134.1 (CH, C-15), 132.9 (C $\left.\mathrm{C}_{\mathrm{q}}, \mathrm{C}-6\right), 129.3$ (CH, C-9), 116.8 (CH, C-5), 98.2 (CH, C1'), 94.0 (C $\mathrm{q}, \mathrm{C}-3), 82.3$ (CH, C-13), 81.9 (CH, C-19), 80.0 (CH, C-7), 77.7 (CH, C-17), 76.7 (CH, C-23), 75.2 (CH, C-3'), 75.0 (CH, C-4'), 71.9 (CH, C-5'), 55.1 (CH, C-24), $43.3\left(\mathrm{CH}_{2}\right.$, C-2), $\left.42.0(\mathrm{CH}, \mathrm{C}-16), 38.0\left(\mathrm{CH}_{2}, \mathrm{C}-26\right), 37.0\left(\mathrm{CH}_{2}, \mathrm{C}-21\right), 36.8\left(\mathrm{CH}_{2}, \mathrm{C}-2\right)\right), 35.1\left(\mathrm{CH}_{2}, \mathrm{C}-\right.$ 12), $35.0\left(\mathrm{CH}_{2}, \mathrm{C}-4\right), 34.4(\mathrm{CH}, \mathrm{C}-18), 33.3(\mathrm{CH}, \mathrm{C}-20), 32.5(\mathrm{CH}, \mathrm{C}-22), 27.0\left(\mathrm{CH}_{2}, \mathrm{C}-10\right)$, $26.0\left(\mathrm{CH}_{2}, \mathrm{C}-11\right), 22.7\left(\mathrm{CH}_{2}, 24-\mathrm{CH}_{2} \mathrm{CH}_{3}\right), 19.2\left(\mathrm{CH}_{3}, 6-\mathrm{CH}_{3}\right), 17.8\left(\mathrm{CH}_{3}, 5{ }^{\prime}-\mathrm{CH}_{3}\right), 17.1$ $\left(\mathrm{CH}_{3}, 16-\mathrm{CH}_{3}\right), 15.8\left(\mathrm{CH}_{3}, 20-\mathrm{CH}_{3}\right), 12.7\left(\mathrm{CH}_{3}, 22-\mathrm{CH}_{3}\right), 11.7\left(\mathrm{CH}_{3}, 24-\mathrm{CH}_{2} \mathrm{CH}_{3}\right), 10.7$ $\left(\mathrm{CH}_{3}, 8-\mathrm{CH}_{3}\right), 7.2\left(\mathrm{CH}_{3}, 27-\mathrm{CH}_{3}\right), 5.4\left(\mathrm{CH}_{3}, 18-\mathrm{CH}_{3}\right)$. 
Cervinomycin $\quad \mathbf{A}_{2} \quad$ (107): $\quad \mathrm{C}_{29} \mathrm{H}_{21} \mathrm{NO}_{9}$ (527), gelber Feststoff, UV-löschend (254 $\mathrm{nm}) .-\boldsymbol{R}_{\boldsymbol{f}}=0.62\left(\mathrm{CH}_{2} \mathrm{Cl}_{2} / \mathrm{MeOH}\right.$ 95:5). Keine Anfärbung. $-\boldsymbol{R}_{\boldsymbol{t}}=17.9 \mathrm{~min}$ (LCMS). $-{ }^{1} \mathbf{H}$ - NMR $\left(\mathrm{CD}_{2} \mathrm{Cl}_{2}, 300 \mathrm{MHz}\right): \delta=$ $13.96(\mathrm{~s}, 1 \mathrm{H}, \mathrm{OH}), 8.19\left(\mathrm{~d},{ }^{3} J=8.5 \mathrm{~Hz}\right.$, $1 \mathrm{H}, 10-\mathrm{H}), 7.96\left(\mathrm{~d},{ }^{3} \mathrm{~J}=8.5 \mathrm{~Hz}, 1 \mathrm{H}, 9-\mathrm{H}\right)$, 7.60 (s, 1H, 19-H), 7.21 (s, 1H, 7-H), 7.14<smiles>COc1cc2oc3c(c(=O)c2cc1OC)C(=O)c1c(ccc2cc4c(c(O)c12)C(=O)N1CCOC1(C)C4)C3=O</smiles>
(s, 1H, 16-H), $4.25\left(\mathrm{t},{ }^{3} J=7.1 \mathrm{~Hz}, 2 \mathrm{H}, 2-\right.$ $\mathrm{H}), 4.04(\mathrm{~m}, 1 \mathrm{H}, 1-\mathrm{H}), 4.00\left(\mathrm{~s}, 3 \mathrm{H}, 17-\mathrm{OCH}_{3}\right), 4.00\left(\mathrm{~s}, 3 \mathrm{H}, 18-\mathrm{OCH}_{3}\right), 3.74(\mathrm{~m}, 1 \mathrm{H}, 1-\mathrm{H})$, $3.32\left(\mathrm{~d},{ }^{3} \mathrm{~J}=14.8 \mathrm{~Hz}, 1 \mathrm{H}, 5-\mathrm{H}\right), 3.23\left(\mathrm{~d},{ }^{3} \mathrm{~J}=14.8 \mathrm{~Hz}, 1 \mathrm{H}, 5-\mathrm{H}\right), 1.41$ (s, 3H, 30-H). $-{ }^{13} \mathrm{C}-$ NMR $\left(\mathrm{CD}_{2} \mathrm{Cl}_{2}, 150.8 \mathrm{MHz}\right): \delta=182.0\left(\mathrm{C}_{\mathrm{q}}, \mathrm{C}-23\right), 178.8\left(\mathrm{C}_{\mathrm{q}}, \mathrm{C}-12\right), 172.7\left(\mathrm{C}_{\mathrm{q}}, \mathrm{C}-21\right), 165.1$ $\left(\mathrm{C}_{\mathrm{q}}, \mathrm{C}-28\right), 161.6\left(\mathrm{C}_{\mathrm{q}}, \mathrm{C}-26\right), 156.2\left(\mathrm{C}_{\mathrm{q}}, \mathrm{C}-17\right), 154.2\left(\mathrm{C}_{\mathrm{q}}, \mathrm{C}-13\right), 151.4\left(\mathrm{C}_{\mathrm{q}}, \mathrm{C}-15\right), 149.4\left(\mathrm{C}_{\mathrm{q}}\right.$, C-18), $141.1\left(\mathrm{C}_{\mathrm{q}}, \mathrm{C}-8\right), 138.2\left(\mathrm{C}_{\mathrm{q}}, \mathrm{C}-24\right), 137.1$ (C $\left.\mathrm{q}, \mathrm{C}-6\right), 132.7$ (CH, C-9), $130.1\left(\mathrm{C}_{\mathrm{q}}, \mathrm{C}-11\right)$, $124.0(\mathrm{CH}, \mathrm{C}-10), 121.2\left(\mathrm{C}_{\mathrm{q}}, \mathrm{C}-22\right), 120.0\left(\mathrm{C}_{\mathrm{q}}, \mathrm{C}-25\right), 119.9\left(\mathrm{C}_{\mathrm{q}}, \mathrm{C}-20\right), 117.9(\mathrm{CH}, \mathrm{C}-7)$, 107.9 (C $\left.\mathrm{C}_{\mathrm{q}}, \mathrm{C}-27\right), 105.1$ (CH, C-19), 100.9 (CH, C-16), 92.5 (C $\left.\mathrm{q}_{\mathrm{q}}, \mathrm{C}-4\right), 64.7\left(\mathrm{CH}_{2}, \mathrm{C}-2\right), 57.0$ $\left(17-\mathrm{OCH}_{3}\right), 56.8\left(18-\mathrm{OCH}_{3}\right), 42.4\left(\mathrm{CH}_{2}, \mathrm{C}-1\right), 41.3\left(\mathrm{CH}_{2}, \mathrm{C}-5\right), 22.9\left(\mathrm{CH}_{3}, \mathrm{C}-30\right)$. - (+)-ESIMS: $m / z(\%)=1077[2 \mathrm{M}+\mathrm{Na}]^{+}(100), 550[\mathrm{M}+\mathrm{Na}]^{+}(18)$.

Anthranilsäureamid (109): $\mathrm{C}_{7} \mathrm{H}_{8} \mathrm{~N}_{2} \mathrm{O}$ (136), farbloser Feststoff, UVlöschend (254 nm), fluoreszierend (366 nm). $-\boldsymbol{R}_{\boldsymbol{f}}=0.35\left(\mathrm{CH}_{2} \mathrm{Cl}_{2} / \mathrm{MeOH}\right.$ 95:5). - Anfärbeverhalten: Anisaldehyd (gelb), Ehrlich (gelb). $-\boldsymbol{R}_{\boldsymbol{t}}=4.2$ $\min (\mathrm{LC}-\mathrm{MS}) .-{ }^{1} \mathbf{H}-\mathbf{N M R}\left(\mathrm{CDCl}_{3}, 300 \mathrm{MHz}\right): \delta=7.36\left(\mathrm{~d},{ }^{3} \mathrm{~J}=8.0 \mathrm{~Hz}\right.$,<smiles>NC(=O)c1ccccc1N</smiles>
$1 \mathrm{H}, 6-\mathrm{H}), 7.23\left(\mathrm{t},{ }^{3} J=8.0 \mathrm{~Hz}, 1 \mathrm{H}, 4-\mathrm{H}\right), 6.68\left(\mathrm{t},{ }^{3} \mathrm{~J}=8.0 \mathrm{~Hz}, 1 \mathrm{H}, 3-\mathrm{H}\right)$, $6.64\left(\mathrm{~d},{ }^{3} J=8.0 \mathrm{~Hz}, 1 \mathrm{H}, 5-\mathrm{H}\right), 5.70\left(\mathrm{~s} \mathrm{br}, 4 \mathrm{H}, 2 \mathrm{NH}_{2}\right) .-$ EI-MS $(70 \mathrm{eV}): m / z(\%)=136$ $\left(\left([\mathrm{M}]^{\circ+}, 91\right), 119(100), 92(50), 65(22)\right.$.

\subsection{Streptomyces sp. GW 51/456}

\subsubsection{Primärscreening}

Der Rohextrakt wies eine intensive Gelbfärbung auf. Dünnschichtchromatographie führte zu keiner klaren Trennung der gebildeten Substanzen. 
Tabelle 15 Aktivität des Rohextraktes von Stamm GW 1909 im biologischen Screening.

\begin{tabular}{|l|c|}
\hline \multicolumn{1}{|c|}{ Testkeim } & Hemmhof $\varnothing[\mathrm{mm}]$ \\
\hline \hline Escherichia coli & 19 \\
\hline Bacillus subtilis & 19 \\
\hline Streptomyces viridochromogenes (Tü 57) & 13 \\
\hline Candida albicans & 12 \\
\hline Mucor miehei (Tü 284) & 0 \\
\hline Staphylococcus aureus & 30 \\
\hline Chlorella sorokiniana & 0 \\
\hline Chlorella vulgaris & 0 \\
\hline Scenedesmus subspicatus & 0 \\
\hline
\end{tabular}

\subsubsection{Kultivierung und Aufarbeitung}

Der Stamm GW1909 wurde auf zwei Agarplatten (Nährmedium $\mathrm{M}_{2}$ ) für $72 \mathrm{~h}$ bei $28^{\circ} \mathrm{C}$ inkubiert. Die so erhaltenen Kulturen wurden zum Beimpfen von sechs mit je $200 \mathrm{ml}$ Medium befüllten 11 Schikanekolben verwendet. Diese Vorkulturen wurden anschließend für $72 \mathrm{~h}$ bei $28^{\circ} \mathrm{C}$ und 95 Upm kultiviert. Die Fermentation der Hauptkulturen erfolgte im 201 Schüttelkultur-Maßstab in 11 Erlenmeyerkolben, welche mit jeweils $200 \mathrm{ml} \mathrm{M}$-Medium befüllt und mit ca. $10 \mathrm{ml}$ der Vorkulturen beimpft wurden. Die anschließende Kultivierung erfolgte auf einem Linearschüttler bei $28^{\circ} \mathrm{C}$ für drei Tage. Direkte Animpfung aus den Agarplatten führte zu keinem guten Wachstum von dem Stamm. Ab zweitem Tag wurde gelb gefärbte Kulturbrühe beobachtet.

Die Kulturbrühe wurde darauf hin mittels einer Filterpresse über Celite filtriert. Das erhaltene Kulturfiltrat wurde an XAD-2 adsorbiert, das Adsorberharz mit 201 Wasser gewaschen und mit 101 Methanol eluiert. Der mit Celite vermischte Zellrückstand wurde dreimal mit Essigester und dreimal mit Aceton unter Ultraschall-Behandlung extrahiert. Die vereinigten organischen Phasen ergaben nach Entfernung des Lösungsmittels 6.8 g Rohextrakt.

\subsubsection{Isolierung und Charakterisierung der Metaboliten}

Der Rohextrakt wurde mittels Säulenchromatographie an Kieselgel mit einem Stufengradienten aus Dichlormethan/Methanol $\left(\mathrm{CH}_{2} \mathrm{Cl}_{2}, \mathrm{CH}_{2} \mathrm{Cl}_{2} / \mathrm{MeOH}\right.$ 99:1, $\mathrm{CH}_{2} \mathrm{Cl}_{2} / \mathrm{MeOH}$ 98:2, $\mathrm{CH}_{2} \mathrm{Cl}_{2} /$ 
MeOH 97:3, $\mathrm{CH}_{2} \mathrm{Cl}_{2} / \mathrm{MeOH}$ 96:4, $\mathrm{CH}_{2} \mathrm{Cl}_{2} / \mathrm{MeOH}$ 95:5, $\mathrm{CH}_{2} \mathrm{Cl}_{2} / \mathrm{MeOH}$ 9:1, $\mathrm{CH}_{2} \mathrm{Cl}_{2} / \mathrm{MeOH}$ 4:1 und $\mathrm{CH}_{2} \mathrm{Cl}_{2} / \mathrm{MeOH}$ 3:2) getrennt. Die zweite Fraktion fiel im Dünnschichtchromatogramm durch eine orange Zone auf und lieferte nach Auftrennung mit Kieselgel und Sepha$\operatorname{dex}(\mathrm{MeOH}) 1.8 \mathrm{mg}$ eines Gemisches aus ß-Indomycinon (111) und Saptomycin A (112). Mehrere Trennungsschritte mittels präparativer Dickschichtchromatographie lieferten schließlich $3.7 \mathrm{mg}$ Thiothymin (110).

Thiothymin (110): $\mathrm{C}_{5} \mathrm{H}_{6} \mathrm{~N}_{2} \mathrm{OS}$ (142), weißer Feststoff, UV-löschend $(254 \mathrm{~nm}) .-\boldsymbol{R}_{\boldsymbol{f}}=0.50\left(\mathrm{CH}_{2} \mathrm{Cl}_{2} / \mathrm{MeOH} 9: 1\right)$. - Keine Anfärbung. $-{ }^{1} \mathbf{H}-$ NMR $\left(\mathrm{CD}_{3} \mathrm{OD}, 300 \mathrm{MHz}\right): \delta=7.84(\mathrm{~s}, 1 \mathrm{H}, 6-\mathrm{H}), 2.31\left(\mathrm{~s}, 3 \mathrm{H}, 5-\mathrm{CH}_{3}\right) .-$ DCI-MS $\left(\mathrm{NH}_{3}\right): m / z(\%)=177\left[\mathrm{M}+\mathrm{NH}_{3}+\mathrm{NH}_{4}\right]^{+}(10), 160\left[\mathrm{M}+\mathrm{NH}_{4}\right]^{+}$ (100), $143[\mathrm{M}+\mathrm{H}]^{+}(35) .-$ EI-MS $(70 \mathrm{eV}): m / z(\%)=142\left(\left([\mathrm{M}]^{++}, 48\right)\right.$,<smiles>Cc1c[nH]c(=O)[nH]c1=S</smiles>
86 (10), 68 (34), 43 (38).

B-Indomycinon (111): $\mathrm{C}_{24} \mathrm{H}_{20} \mathrm{O}_{6} \quad$ (404), oranger Feststoff, UV-löschend (254 nm) und orange fluoreszierend (336 nm). $-\boldsymbol{R}_{\boldsymbol{f}}=0.75\left(\mathrm{CH}_{2} \mathrm{Cl}_{2} / \mathrm{MeOH} 95: 5\right)$. - Keine Anfärbung. $-\boldsymbol{R}_{\boldsymbol{t}}=21.8 \mathrm{~min}(\mathrm{LC}-\mathrm{MS}) .-{ }^{\mathbf{1}} \mathbf{H}-$ NMR $\left(\mathrm{CDCl}_{3}, 600 \mathrm{MHz}\right): \delta=12.84$ (s br, $\left.1 \mathrm{H}, \mathrm{OH}\right)$, $8.06(\mathrm{~s}, 1 \mathrm{H}, 6-\mathrm{H}), 7.81\left(\mathrm{~d},{ }^{3} \mathrm{~J}=7.5 \mathrm{~Hz}, 1 \mathrm{H}, 8-\mathrm{H}\right), 7.67$ $\left(\mathrm{t},{ }^{3} J=8.0 \mathrm{~Hz}, 1 \mathrm{H}, 9-\mathrm{H}\right), 7.34\left(\mathrm{~d},{ }^{3} J=8.4 \mathrm{~Hz}, 1 \mathrm{H}\right.$,<smiles></smiles>
10-H), 6.51 (s, 1H, 3-H), $5.70(\mathrm{~m}, 1 \mathrm{H}, 18-\mathrm{H}), 5.35$ (m, 1H, 17-H), 3.02 (s, 3H, 13-H), $2.90\left(\mathrm{dd},{ }^{2} J=14.3 \mathrm{~Hz},{ }^{3} J=6.7 \mathrm{~Hz}, 1 \mathrm{H}, 16-\mathrm{H}_{\mathrm{A}}\right), 2.76$ (s br, 1H, OH), $2.75\left(\mathrm{~m}, 1 \mathrm{H}, 16-\mathrm{H}_{\mathrm{B}}\right), 1.68(\mathrm{~s}, 3 \mathrm{H}, 15-\mathrm{H}), 1.62\left(\mathrm{~d},{ }^{3} J=6.8 \mathrm{~Hz}, 3 \mathrm{H}, 19-\mathrm{H}\right) .-(+)-\mathbf{E S I}-$ MS: $m / z(\%)=831[2 \mathrm{M}+\mathrm{Na}]^{+}, 405[\mathrm{M}+\mathrm{H}]^{+}$.

Saptomycin-A (112): $\mathrm{C}_{24} \mathrm{H}_{20} \mathrm{O}_{6} \quad$ (404), oranger Feststoff, UV-löschend (254 nm) und orange fluoreszierend (366 nm). $-\boldsymbol{R}_{\boldsymbol{f}}=0.70\left(\mathrm{CH}_{2} \mathrm{Cl}_{2} / \mathrm{MeOH} 95: 5\right)$. - Keine Anfärbung mit Anisaldehyd und Ehrlich. $\boldsymbol{R}_{\boldsymbol{t}}=20.7 \min (\mathrm{LC}-\mathrm{MS}) .-{ }^{\mathbf{1}} \mathbf{H}-\mathbf{N M R}\left(\mathrm{CDCl}_{3}, 600\right.$ $\mathrm{MHz}): \delta=12.63(\mathrm{~s} \mathrm{br}, 1 \mathrm{H}, \mathrm{OH}), 8.07(\mathrm{~s}, 1 \mathrm{H}, 6-\mathrm{H})$, $7.81\left(\mathrm{~d},{ }^{3} \mathrm{~J}=7.5 \mathrm{~Hz}, 1 \mathrm{H}, 8-\mathrm{H}\right), 7.67\left(\mathrm{t},{ }^{3} \mathrm{~J}=8.0 \mathrm{~Hz}\right.$, $1 \mathrm{H}, 9-\mathrm{H}), 7.34\left(\mathrm{~d},{ }^{3} J=8.4 \mathrm{~Hz}, 1 \mathrm{H}, 10-\mathrm{H}\right), 6.25$ (s,<smiles>C/C=C/C(O)C(C)c1cc(=O)c2c(C)cc3c(c2o1)C(=O)c1c(O)cccc1C3=O</smiles>
$1 \mathrm{H}, 3-\mathrm{H}), 5.64(\mathrm{~m}, 1 \mathrm{H}, 18-\mathrm{H}), 5.49(\mathrm{~m}, 1 \mathrm{H}, 17-\mathrm{H}), 5.00(\mathrm{~m}, 1 \mathrm{H}, 16-\mathrm{H}), 3.49$ (br d, 1H, OH), 
$3.02(\mathrm{~s}, 3 \mathrm{H}, 13-\mathrm{H}), 2.97$ (m, 1H, 14-H), $1.68\left(\mathrm{~d},{ }^{3} \mathrm{~J}=7.0 \mathrm{~Hz}, 3 \mathrm{H}, 19-\mathrm{H}\right), 1.43\left(\mathrm{~d},{ }^{3} \mathrm{~J}=7.0 \mathrm{~Hz}\right.$, 3H, 15-H). - (+)-ESI-MS: $m / z(\%)=831[2 \mathrm{M}+\mathrm{Na}]^{+}, 405[\mathrm{M}+\mathrm{H}]^{+}$.

\subsection{Streptomyces sp. Mei24}

\subsubsection{Primärscreening}

Das grün gefärbte Rohextrakt des Nordsee-Bakteriums zeigte im Dünnschichtchromatogramm zeigte beim Tageslicht zahlreiche blaue und violette Zonen. Die farblosen und nicht UV-löschenden Zonen wurden mit Anisaldehyd/Schwefelsäure bei der Wärme in verschiedene Farben angefärbt.

Tabelle 16 Aktivität des Rohextraktes von Streptomyces sp. Mei24 im biologischen Screening.

\begin{tabular}{|l|c|}
\hline \multicolumn{1}{|c|}{ Testkeim } & Hemmhof $\varnothing[\mathrm{mm}]$ \\
\hline \hline Bacillus subtilis & 11 \\
\hline Staphylococcus aureus & 27 \\
\hline Streptomyces viridochromogenes (Tü 57) & 0 \\
\hline Escherichia coli & 0 \\
\hline Candida albicans & 13 \\
\hline Mucor miehei (Tü 284) & 11 \\
\hline Chlorella vulgaris & 26 \\
\hline Chlorella sorokiniana & 25 \\
\hline Scenedesmus subspicatus & 27 \\
\hline
\end{tabular}

\subsubsection{Kultivierung und Aufarbeitung}

Die Kultivierung von Stamm Mei 24 erfolgte im 201 Schüttelkultur-Maßstab in 11 Erlenmeyerkolben, welche mit je $200 \mathrm{ml}$ Mei-Stamm-Medium befüllt und mit einem $1 \mathrm{~cm}^{2}$ großen Stück einer gut bewachsenen Agarplatte beimpft wurden. Die Fermentation wurde auf einem Linearschüttler bei $28^{\circ} \mathrm{C}$ für sechs Tage durchgeführt.

Die Aufarbeitung der Kulturen erfolgte wie in Kapitel 10.8.2 beschrieben. Es ergaben sich so $1.7 \mathrm{~g}$ Rohextrakt. 


\subsubsection{Isolierung und Charakterisierung der Metaboliten}

Der Rohextrakt wurde mittels Säulenchromatographie an Kieselgel mit einem Stufengradienten aus Dichlormethan/Methanol $\left(\mathrm{CH}_{2} \mathrm{Cl}_{2}, \mathrm{CH}_{2} \mathrm{Cl}_{2} / \mathrm{MeOH}\right.$ 99:1, $\mathrm{CH}_{2} \mathrm{Cl}_{2} / \mathrm{MeOH} 98: 2, \mathrm{CH}_{2} \mathrm{Cl}_{2}$ l $\mathrm{MeOH}$ 97:3, $\mathrm{CH}_{2} \mathrm{Cl}_{2} / \mathrm{MeOH}$ 96:4, $\mathrm{CH}_{2} \mathrm{Cl}_{2} / \mathrm{MeOH}$ 95:5, $\mathrm{CH}_{2} \mathrm{Cl}_{2} / \mathrm{MeOH}$ 9:1 und $\mathrm{CH}_{2} \mathrm{Cl}_{2} /$ $\mathrm{MeOH} 4: 1)$ getrennt. Weitere chromatographische Aufreinigung der zweiten Fraktion an Kieselgel (Cyclohexan/EE) lieferte $0.8 \mathrm{mg}$ 4-Methylamino-7-methyl-isoquinolin-3,6-dion (113) und $0.8 \mathrm{mg}$ 4-Methylamino-7,8-dimethyl-isoquinolin-3,6-dion (114). Die dritte Fraktion ergab nach Chromatographie an Kieselgel $\left(\mathrm{CH}_{2} \mathrm{Cl}_{2} / \mathrm{MeOH}\right) 346.5 \mathrm{mg}$ eines Fettsäure-Gemischs. Die restlichen Fraktionen führten nach mehreren chromatographischen Trennschritten an Kieselgel sowie Sephadex zu 0.6 mg Indolcarbonsäure (33), 0.4 mg 3-Hydroxyacetylindol (34), 2.4 mg Uracil (35) und 6.5 mg p-Hydroxybenzoesäure (36).

\section{4-Methylamino-7-methyl-isoquinolin-3,6-dion (113):}

$\mathrm{C}_{11} \mathrm{H}_{10} \mathrm{~N}_{2} \mathrm{O}_{2}$ (202), roter Feststoff, UV-löschend (254 nm). $-\boldsymbol{R}_{\boldsymbol{f}}$ $=0.67\left(\mathrm{CH}_{2} \mathrm{Cl}_{2} / \mathrm{MeOH}\right.$ 9:1). - Anfärbeverhalten: Ehrlich (orange). $-\boldsymbol{R}_{\boldsymbol{t}}=11.4 \mathrm{~min}$ (LC-MS). $-{ }^{1} \mathbf{H}-\mathbf{N M R}\left(\mathrm{CDCl}_{3}, 600\right.$<smiles>CNC1=CC(=O)c2cc(C)ncc2C1=O</smiles>
MHz): $\delta=9.13(\mathrm{~s}, 1 \mathrm{H}, 2-\mathrm{H}), 7.75(\mathrm{~s}, 1 \mathrm{H}, 7-\mathrm{H}), 6.06$ (s br, $1 \mathrm{H}$, 4-NH), 5.77 (s, 1H, 5-H), 2.94 (d, $\left.{ }^{3} J=4.6 \mathrm{~Hz}, 3 \mathrm{H}, 4-\mathrm{NHCH}_{3}\right), 2.70$ (s, 3H, 7-H).

\section{4-Methylamino-7,8-dimethyl-isoquinolin-3,6-dion}

(114):

$\mathrm{C}_{12} \mathrm{H}_{12} \mathrm{~N}_{2} \mathrm{O}_{2}$ (216), roter Feststoff, UV-löschend (254 nm). $-\boldsymbol{R}_{\boldsymbol{f}}$ $=0.70\left(\mathrm{CH}_{2} \mathrm{Cl}_{2} / \mathrm{MeOH}\right.$ 9:1). - Anfärbeverhalten: Ehrlich (orange). $-\boldsymbol{R}_{\boldsymbol{t}}=13.9 \mathrm{~min}$ (LC-MS). $-{ }^{\mathbf{1}} \mathbf{H}-\mathbf{N M R}\left(\mathrm{CDCl}_{3}, 600\right.$ $\mathrm{MHz}): \delta=9.00(\mathrm{~s}, 1 \mathrm{H}, 2-\mathrm{H}), 5.79$ (s br, 1H, 4-NH), 5.70 (s,<smiles>CNC1=CC(=O)c2c(cnc(C)c2C)C1=O</smiles>
1H, 5-H), $2.90\left(\mathrm{~d},{ }^{3} \mathrm{~J}=4.6 \mathrm{~Hz}, 3 \mathrm{H}, 4-\mathrm{NHCH}_{3}\right), 2.73$ (s, 3H, 8-H), 2.68 (s, 3H, 7-H). - (+)ESI-MS: $m / z(\%)=217[\mathrm{M}+\mathrm{H}]^{+}(15)$. 


\section{Literaturverzeichnis}

1 M. H. G. Munro, J. W. Blunt, MarinLit Database 2002, University of Canterbury.

2 D. J. Newman, G. M. Cragg, Curr. Med. Chem. 2004, 11, 1693-1713.

3 A. M. S. Mayer, K. R. Gustafson, Int. J. Cancer 2003, 105, 291-299.

4 K. L. Rinehart, J. B. Gloer, R. G. Hughes, H. E. Renis, J. P. Mcgovren, E. B. Swynenberg, D. A.

Stringfellow, S. L. Kuentzel, L. H. Li, Science 1981, 212, 933-935.

5 G. R. Pettit, C. L. Herald, D. L. Doubek, D. L. Herald, E. Arnold, J. Clardy, J. Am. Chem. Soc. 1982, 104, 6846-6848.

6 M. G. Haygood, S. K. Davidson, Appl. Env. Microbiol. 1997, 63, 4612-4616.

7 M. Murata, H. Naoki, T. Iwashita, S. Matsunaga, M. Sasaki, A. Yokoyama, T. Yasumoto, J. Am. Chem. Soc. 1993, 115, 2060-2062.

8 T. Nonomura, M. Sasaki, N. Matsumori, M. Miata, K. Tachibana, T. Yasumoto, Angew. Chem., Int. Ed. Engl. 1996, 35, 1675-1678.

9 M. Spindler, G. S. Dieckmann, Spektrum der Wissenschaft, Februar 1991, 48-57.

10 D. Schröder, Dissertation, Universität Göttingen 2001.

11 C. A. Volk, H. Lippert, E. Lichte, M. Koeck, Eur. J. Org. Chem. 2004, 14, 3154-3158.

12 J. Voss, K. Taraz, H. Budzikiewicz, Z. Naturforsch. C 1999, 54, 156-162.

13 Römpp Chemie Lexikon, 4. Auflage, (Hrsg.: J. Falbe, M. Regitz), Thieme Verlag, Stuttgart/New York, 1990.

14 C. Burk, E. Usleber, E. Martlbauer, Arch. Lebensmittelhyg. 1998, 49, 16-20.

15 M. Kodama, T. Ogata, S. Sato, Agric. Biol. Chem. 1988, 52, 1075-1077.

16 S. Gallacher, E. A. Smith, Protist 1999, 150, 245-255.

17 M. S. Gil-Turnes, M. E. Hay, W. Fenical, Science 1989, 246, 116-118.

18 M. S. Gil-Turnes, W. Fenical, Biol. Bull. 1992, 182, 105.

19 G. M. Lipkind, H. A. Fozzard, Biophys. J. 1994, 66, 1-13.

20 K. Miyazawa, T. Noguchi, J. Toxicol.-Toxin Rev. 2001 20, 11-33.

21 U. Simidu, T. Noguchi, D. F. Hwang, Y. Shida, K. Hashimoto, Appl. Environ. Microbiol. 1987, 53, 17141715.

22 M. J. Lee, D. Y. Jeong, W. S. Kim, H. D. Kim, C. H. Kim, W. W. Park, Y. H. Park, K. S. Kim, H. M. Kim, D. S. Kim, Appl. Environ. Microbiol. 2000, 66, 1698-1701.

23 E. D. Weinberg, Perspect. Biol. Med. 1993, 36, 215-221.

24 K. G. Wooldridge, P. H. Williams, FEMS Microbiol. Rev. 1993, 12, 325-348.

25 B. E. Britigan, G. T. Rasmussen, C. D. Cox, Infect. Immun. 1997, 65, 1071-1076.

26 Y. Dessaux, A. Petit, J. Tempe, Phytochem. 1993, 34, 31-38.

27 J. Köppel, J. Tenczer, J. Am. Soc. Mass Spectrom. 1995, 6, 995-1003.

28 P. G. M. Kienhuis, R. B. Geerdink, J. Chromatogr. A 2002, 974, 161-168.

29 L. L. Lopez, P. R. Tiller, M. W. Senko, J. C. Schwartz, Rapid. Commun. Mass Spectrom. 1999, 13, $663-668$.

30 Laatsch, H. AntiBase 2002, A Natural Products Database for Rapid Structure Determination. Chemical Concepts, Weinheim 2002; see Internet http://www.gwdg.de/ ucoc/laatsch/. 
31 Autorenkollektiv, Chapman \& Hall/CRC Dictionary of Natural Products on CD-ROM, Version 12:1, Chapman \& Hall, CRC, 2003.

32 M. R. Wilkins, C. Pasquali, R. D. Appel, K. Ou, O. Golaz, J. C. Sanchez, J. X. Yan, A. A. Gooley, G. Hughes, I. HumphreySmith, K. L. Williams, D. F. Hochstrasser, BioTechnology 1996, 14, 61-65.

33 P. Edman, Arch. Biochem. Biophys. 1949, 22, 475-483.

34 P. Roepstorff, J. Fohlmann, Biomed. Mass Spectrom. 1984, 11, 601.

35 K. Vekey, J. Mass Spectrom. 1996, 31, 445-463.

36 Z. Tozuka, H. Kaneko, T. Shiraga, Y. Mitani, M. Beppu, S. Terashita, A. Kawamura, A. Kagayama, J. Mass Spectrom. 2003, 38, 793-808.

37 D. A. McCrery, M. L. Gross, Anal. Chim. Acta 1985, 178, 91-103.

38 A. Reis, M. R. M. Domingues, P. Domingues, A. J. Ferrer-Correia, M. A. Coimbra, Carbohydr. Res. 2003, $338,1497-1505$.

39 S. A. Waksman, H. B. Woodruff, Proc. Soc. Biol. Med. 1940, 45, 609-614.

40 H. Brockmann, H. Lackner, Naturwissenschaften 1960, 47, 230-231.

41 H. Lackner, Angew. Chem. 1975, 87, 400-411.

42 A. Mauger, E. Katz, Arch. Biochem. Biophys. 1976, 176, 181-186.

43 H. Schulten in Soft Ionization Biological Mass Spectrometry, (Hrsg.: H. Morris), Heyden, London, 1981, s. 27.

44 A. B. Mauger, O. A. Stuart, J. A. Ferretti, J. V. Silverton, J. Am. Chem. Soc. 1985, 107, 7154-7163.

45 J. Greaves, M. McCamish, J. Roboz, Proceedings of the $32^{\text {nd }}$ ASMS Conference in Mass Spectrometry and Allied Topics, San Antonio, TX, 1984, s. 447.

46 M. Barber, D. Bell, M. Morris, L. Tetler, M. Woods, B. W. Bycroft, J. J. Monaghan, W. E. Morden, B. N. Green, Talanta 1988, 35, 605-611.

47 J. Roboz, E. Nieves, J. F. Holland, M. McCamish, C. Smith, Biomed. Environ. Mass Spectrom. 1988, 16, 6770.

48 D. Thomas, M. Morris, J. M. Curtis, R. K. Boyd, J. of Mass Spectrom. 1995, 30, 1111-1125.

49 D. W. Russel, J. Chem. Soc. 1962, 753-761.

50 C. G. Macdonald, J. S. Shannon, Tetrahedron Lett. 1964, 31, 2087-2092.

51 B. C. Das, P. Varenne, A. Taylor, J. Antibiot. 1979, 32, 569-574.

52 H. A. Gillis, D. W. Russell, A. Taylor, J. A. Walter, Can. J. Chem. 1990, 68, 19-21.

53 M. S. Gil-Turnes, M. E. Hay, W. Fenical, Science 1989, 246, 116-118.

54 L. Liang, Dissertation 2003, Universität Göttingen.

55 H. P. Zhang, H. Shigemori, M. Ishibashi, T. Kosaka, G. R. Pettit, Y. Kamano, J. Kobayashi, Tetrahedron 1994, 50, 10201-10206.

56 H. P. Zhang, Y. Kamano, Y. Ichihara, H. Kizu, K. Komiyama, H. Itokawa, G. R. Pettit, Tetrahedron 1995, $51,5523-5528$.

57 Y. Kamano, H. P. Zhang, Y. Ichihara, H. Kizu, K. Komiyama, G. R. Pettit, Tetrahedron Lett. 1995, 51, 55235528.

58 J. D. Bu'Lock, D. Drake, D. J. Winstanley, Phytochem. 1972, 11, 2011-2018.

59 R. Meganathan, Cell. Molec. Biol. 1996, 642-656. 
60 M. S. Gill-Tunes, Dissertation, San Diego 1988.

61 M. D. Collins, D. Jones, Microbiol. Rev. 1981, 45, 316-354.

62 R. A. Morton in In Quinones in Electron Transport, (Hrsg.: G. E. W. Wolstenholme und C. M. O’Connor), Churchill, London, 1961, 5-35.

63 F. L. Crane in In Quinones in Electron Transport, (Hrsg.: G. E. W. Wolstenholme und C. M. O’Connor), Churchill, London, 1961, 36-78.

64 B. Soballe, R. K. Poole, Microbiol. 1999, 145, 1817-1830.

65 J. R. F. Allen, B. R. Holmstedt, Phytochem. 1980, 19, 1573-1582.

66 K. Yomosa, A. Hirota, H. Sakai, A. Isogai, J. Agric. Biol. Chem. 1987, 51, 921-922.

67 S. Inoue, K. Okuda, H. Tanino, H. Kakoi, T. Goto, Chem. Lett. 1980, 297-298.

68 G. Habermehl, P. Hammann, Naturstoffchemie, Springer-Verlag, Berlin, 1992, s. 151.

69 X. Fu, F. J. Schmitz, R. S. Tanner, J. Nat. Prod. 1995, 58, 1950-1954.

70 M. Lang, P. Spiteller, V. Hellwig, W. Steglich, Angew. Chem, Int. Ed. 2001, 40, 1704-1705.

71 H. Zhang, W. Jin, Y. Wang, Zhongguo Kangshengsu Zazhi 1990, 15, 321-324.

72 T. Morita, H. Aoki, Agric. Biol. Chem. 1974, 38, 1501-1505.

73 R. T. Sherwood, US Patent No. 3179653 (1962); Chem. Abstr. 1965, 63, 1170.

74 J. R. Dimmock, J. Sci. Food Agric. 1967, 18, 368-372.

75 S. I. Sviridov, B. S. Ermolinskii, Chem. Nat. Comp. 1990, 26, 691-696.

76 A. K. Bose, Z. Urbanczyk-Lipkowska, G. V. Subbaraju, M. S. Manhas, S. N. Ganguly, Oceanogr. Indian Ocean 1992, 407-411.

77 Z. Urbańczyk-Lipkowska, M. Y. Lee, Pol. J. Chem. 1992, 66, 1805-1811.

78 R. Jiang, B. Li, C. Xiao, D. Yang, J. Wu, Zhongguo Kangshengsu Zazhi 1997, 22, 81-83, 139.

79 D. Shangguan, R. Jiang, B. Li, C. Xiao, J. Wu, Zhongguo Kangshengsu Zazhi 1999, 24, 254-257, 299.

80 S. Omura, Y. Iwai, A. Hirano, A. Nakagawa, J. Awaya, H. Tsuchiya, Y. Takahashi, R. J. Masuma, J. Antibiot. 1977, 30, 275-282.

81 S. -W. Yang, G. A. Cordell, J. Nat Prod. 1997, 60, 44-48.

82 K. Ohta, Proceedings-International Seaweed Symposium 1979, 9, 401-411.

83 K. Ohta, M. Takagi, Phytochem. 1977, 16, 1085-1086.

84 P. Mende, M. Siddiqi, R. Preussmann, B. Spiegelhalder, Cancer Lett. 1994, 83, 277-282.

85 G. A. Cordell, P. T. O. Chang, H. H. S. Fong, N. R. Farnsworth, Lloydia 1977, 40, 340-343.

86 G. C. Robinson, U.S. 1970, 5 pp.

87 M. Siddiqueullah, R. McGrath, L. C. Vining, F. Sala, D. W. S. Westlake, Can. J. Biochem. 1967, 45, 18811889.

88 R. Cardillo, C. Fuganti, D. Ghiringhelli, D. Giangrasso, P. Grasselli, A. Santopietro-Amisano, Tetrahedron 1974, 30, 459-461.

89 C. J. Chang, H. G. Floss, D. J. Hook, J. A. Mabe, P. E. Manni, L. L. Martin, K. Schroder, T. L. Shieh, J. Antibiot. 1981, 34, 555-566.

90 S. Kirner, K.-H. van Pée, Angew. Chem. 1994, 106, 346-347.

91 G. T. Carter, J. A. Nietsche, J. J. Goodman, M. J. Torrey, T. S. Dunne, M. M. Siegel, D. B. Borders, J. Chem. Soc., Chem. Commun. 1989, 1271-1273. 
92 Y. Li, S. J. Gould, P. J. Proteau, Tetrahedron Lett. 2000, 41, 5181-5185.

93 J. Yinon, M. Laschever, Org. Mass Spectrom. 1981, 16, 264-266.

94 A. G. Harrison, R. K. M. R. Kallury, Org. Mass Spectrom. 1980, 15, 284-288.

95 J. J. Brophy, V. Diakiw, R. J. Goldsack, D. Nelson, J. Shannon, Org. Mass Spectrom. 1979, 14, $201-203$.

96 T. Karancsi, P. Slegel, J. Mass Spectrom. 1999, 34, 975-977.

97 R. G. Gillis, M. J. Lacey, J. S. Shannon, Org. Mass Spectrom. 1974, 9, 359-364.

98 R. Surette, L. C. Vining, J. Antibiot. 1976, 29, 646-652.

99 R. G. Ackman, C. S. Tocher, J. McLachan, J. Fish. Res. Board Can. 1966, 23, 357-364.

100 D. C. Yoch, Appl. Environ. Microbiol. 2002, 68, 5804-5815.

101 P. Mayser, W.Steglich, H.-J. Kraemer, B. Irlinger, Ger. Offen. 2002.

102 R. H. White, J. Bacteriol. 1987, 169, 5859-5860.

103 M. R. Hansen, L. H. Hurley, Acc. Chem. Res. 1996, 29, 249-258.

104 K. W. Glombitza, Archiv für Mikrobiol. 1967, 57, 39-42.

105 P. Fenn, R. D. Durbin, J. E. Kuntz, Phytochem. 1977, 16, 899-901.

106 R. P. Maskey, P. N. Asolkar, E. Kapaun, I. Wagner-Döbler, H. Laatsch, J. Antibiot. 2002, 55, 643-649.

107 O. S. Kwon, S. H. Park, B.-S. Yun, Y. R. Pyun, C.-J. Kim, J. Antibiot. 2000, 53, 954-958.

108 J. Watanabe, J. Kawabata, H. Kurihara, R. Niki, Biosci. Biotech. Biochem. 1997, 61, 177-178.

109 H. Cramer, W. Pfleiderer, Helv. Chim. Acta 1996, 79, 2114-2136.

110 N. Miyairi, H. I. Sakai, T. Konomi, H. Imanaka, J. Antibiot. 1976, 29, 227-235.

111 N. Sitachitta, M. Gadepalli, B. S. Davidson, Tetrahedron 1996, 52, 8073-8080.

112 H. Kang, P. R. Jensen, W. Fenical, J. Org. Chem. 1996, 61, 1543-1546.

113 P. Babczinski, M. Dorgerloch, A. Lobberding, H. J. Santel, R. R. Schmidt, P. Schmitt, C. Wunsche, Pestic. Sci. 1991, 33, 439-446.

114 B. S. Moore, C. Hertweck, Nat. Prod. Rep. 2002, 19, 70-99.

115 H. Seto, T. Sato, S. Urano, J. Uzawa, H. Yonehara, Tetrahedron Lett. 1976, 4367-4370.

116 J. Piel, K. Hoang, B. S. Moore, J. Am. Chem. Soc. 2000, 122, 5415-5416.

117 J. Piel, C. Hertweck, P. R. Shipley, D. M. Hunt, M. S. Newman, B. S. Moore, Chem. Biol. 2000, 7, 943-955.

118 C. E. Salomon, N. A. Magarvey, D. H. Sherman, Nat. Prod. Rep. 2004, 21, 105-121.

119 A. Gogoll, D. Tanner, Magnet. Reson. Chem. 1989, 27, 863-871.

${ }^{120}$ M. Brufani, W. Keller-Schierlein, W. Löffler, I. Mansperger, H. Zähner, Helv. Chim. Acta 1968, 51, 1293.

121 S. Neunlist, P. Bisseret, M. Rohmer, Eur. J. Biochem. 1988, 171, 245-252.

122 M. Rohmer, P. Bouvier, G. Ourisson, J. Gen. Microbiol. 1984, 130, 1137-1150.

123 M. Rohmer, P. Bouvier, G. Ourisson, Proc. Nat. Acad. Sci. USA 1979, 76, 847-851.

124 G. Ourisson, M. Rohmer, K. Poralla, Annu. Rev. Microbiol. 1987, 41, 301-303.

125 D. C. Jordan, Int. J. Syst. Bacteriol. 1982, 32, 136-139.

126 A. M. Berry, O. T. Harriott, R. A. Moreau, S. F. Osman, D. R. Benson, A. D. Jones, Proc. Nat. Acad. Sci. USA 1993, 90, 6091-6094.

127 M. Lemoigne, Ann. Inst. Pasteur 1925, 39, 144.

128 M. Lemoigne, Bull. Soc. Chim. Biol. 1926, 8, 770-782; Ann. Inst. Pasteur 1927, 41, 148.

${ }^{129}$ V. H.-M. Müller, D. Seebach, Angew. Chem. 1993, 105, 483-509. 
130 M. Doudoroff, R. Y. Stainer, Nature 1959, 183, 1440-1442.

131 R. P. Maskey, I. Kock, M. Shaaban, I. Grün-Wollny, E. Helmke, F. Mayer, I. Wagner-Döbler, H. Laatsch, Polym. Bull. 2002, 49, 87-93.

132 T. Itoh, M. Kinoshita, S. Aoki, M. Kobayashi, J. Nat. Prod. 2003, 66, 1373-1377.

133 E. Heinz in Lipids and Lipid Polymers in Higher Plants, (Hrsg.: M. Tevini, H. K. Lichtenthaler), Springer, Berlin, Heidelberg und New York, 1997, s. 102-115.

134 B. W. Nicolas in The Biology of blue-green algae, (Hrsg.: N. G. Carr, B. A. Whitton), University of California Press, Berkeley und Los Angeles, 1973, s. 144-161.

135 J. Shirakibura, N. Murakami, M. Watanabe, A. Nagatsu, J. Sakakibara, H. Tokuda, H. Nishino, A. Iwashima, Chem. Pharm. Bull. 1993, 41, 1664-1666.

${ }^{136}$ H. Kikuchi, Y. Tsukitani, T. Manda, T. Fujii, H. Nakanishi, M. Kobayashi, I. Kitagawa, Chem. Pharm. Bull. 1982, 30, 3544-3547.

137 N. Murakami, H. Imamura, J. Sakakibara, N. Yamada, Chem. Pharm. Bull. 1990, 38, 3497-3499.

138 A. Mitsui, D. Rosner, A. Goodman, G. Reyes-Vasques in Red Tides. Biology, Environmental Science, and Toxicology, (Hrsg.: T. Okaichi, D. M. Anderson, T. Nemoto), Elsevier, New York, 1989, s. 367-370.

139 D. Gordon, S. J. Danishefsky, J. Am. Chem. Soc. 1992, 114, 659-663.

${ }^{140}$ S. Omura, A. Nakagawa, N. Imamura, K. Kushida, C.-M. Liu, L. H. Sello, J. W. Westley, J. Antibiot. 1985, $38,674-676$.

141 J. W. Westley, C.-M. Liu, L. H. Sello, R. H. Evans, N. Troupe, J. F. Blount, A. M. Chiu, L. J. Todaro, P. A. Miller, J. Antibiot. 1984, 37, 1738-1740.

142 S. Omura, N. Imamura, K. Hinotozawa, K. Otoguro, G. Lukacs, R. Faghih, R. Tolmann, B. H. arison, J. L. smith, J. Antibiot. 1983, 36, 1783-1786.

${ }^{143}$ G. Werner, H. Hagenmaier, K. Albert, H. Kohlshorn, H. Drautz, Tetrahedron Lett. 1983, 24, 5193-5196.

144 A. Nakagawa, S. Omura, J. Antibiot. 1987, 40, 301-308.

145 S. Omura, Y. Iwai, K. Hinotozawa, Y. Takahashi, J. Kato, A. Nakagawa, A. Hirano, H. Shimizu, K. Haneda, J. Antibiot. 1982, 35, 645-652.

146 A. Nakagawa, Y. Iwai, H. Shimizu, S. Omura, J. Antibiot. 1986, 39, 1636-1638.

147 M. R. Hansen, L. H. Hurley, Acc. Chem. Res. 1996, 29, 249-258.

148 N. Abe, Y. Nakakita, T. Nakamura, N. Enoki, H. Uchida, M. Munekata, J. Antibiot. 1993, 46, 1530-1535.

149 M. Speitling, Dissertation 1998, Universität Göttingen.

${ }^{150}$ M. A. Shaaban Mahmoud, Dissertation 2004, Universität Göttingen.

${ }^{151}$ M. M. Joullie, J. K. Puthenpurayil, J. Heterocyc. Chem. 1969, 6, 697-705.

${ }^{152}$ G. R. Pettit, J. C. Collins, D. L. Herald, D. L. Doubek, M. R. Boyd, J. M. Schmidt, J. N. A. Hooper, L. P. Tackett, Can. J. Chem. 1992, 70, 1170-1175. 


\section{Danksagung}

Ein großes Dankeschön richtet sich an Dr. H. Frauendorf, dessen exzellente Betreuung in massenspektrometrischen Fragen maßgeblich zum Gelingen dieser Arbeit beigetragen hat. Für die Kultivierung und das biologische Screening der polaren Stämme danke ich Wael Al Zereini und Prof. Dr. H. Anke (Institut für Biotechnologie und Wirkstoffforschung, Kaiserslautern). Dr. E. Helmke (Alfred-Wegener-Institut, Bremerhaven) möchte ich für die Bereitstellung der arktischen und antarktischen Bakterienstämme danken.

Dr. I. Grün-Wollny (Institut für Bodenkunde, Lohra-Kirchvers) und Prof. Dr. M. Meiners (Fachhochschule Ostfriesland, Emden) gilt mein Dank für die Bereitstellung der terrestrischen bzw. marinen Streptomyceten.

Bei Herrn Reinhard Machinek, Carola Zolke, Christiane Siebert und Martin Weitemeyer bedanke ich mich für das oft zeitraubende Messen der vielen NMR-Spektren.

Für die Aufnahme der Massenspektren möchte ich mich bei Györgyi Udvarnoki und Gabriele Krökel bedanken.

Friederike Lissy danke ich für die Unterstützung bei mikrobiologischen Arbeiten. Yvonne Bartoschek und Avan Taha gilt mein Dank für UV- und IR-Messungen sowie das chemische Screening von Bakterienstämmen. Andreas Kohl möchte ich für die Herstellung von PDC-Platten und für die Hilfe bei technischen Problemen danken.

Bei Olaf Senge und Frank Hambloch bedanke ich mich für die Unterstützung bei den HPLC- und GC-MS-Messungen.

Jens Bitzer, Prof. Dr. A. Zeeck und Prof. Dr. H. Lackner gilt mein Dank für die Überlassung der Actinomycine.

Marc Corbella und Isis Ricarno danke ich für die Synthesen der Nitroverbindungen.

Bei allen Mitgliedern der Arbeitsgruppe Laatsch möchte ich mich herzlich für das gute Arbeitsklima, die fruchtbaren Diskussionen und die vielen lustigen Stunden außerhalb der Chemie bedanken.

Großer Dank gilt meinen Eltern, die stets ihr möglichstes getan haben, mich zu unterstützen und - trotz der großen räumlichen Distanz - immer für mich da waren.

Mein ganz besonderer Dank gilt meinem Mann Tim für das sorgfältige Korrekturlesen dieses Manuskripts und die ständige Unterstützung in guten sowie schwierigen Zeiten. 


\section{Lebenslauf}

Am 03.11.1975 wurde ich als erstes Kind von Lianny Irawati und Putu Laksana Oka in Surabaya, Indonesien geboren.

Von Juli 1982 bis Juni 1988 besuchte ich die St. Don Bosco-Grundschule in Jakarta, Indonesien. Danach wechselte ich zur St. Theresia-Mittelschule (Jakarta), wo ich bis zum Juni 1991 blieb. Anschließend war ich Schülerin der St. Theresia-Oberschule (Jakarta), die ich im Juni 1994 mit Bestehen der Abschlussprüfung verließ.

Von Oktober 1994 bis Juli 1995 besuchte ich im Rahmen der Vorbereitung für ein Studium an einer deutschen Hochschule das Studienkolleg in München.

Zum Wintersemester 1995/1996 begann ich das Studium der Chemie an der Universität Bayreuth, wo ich im Januar 1998 die Diplom-Chemiker-Vorprüfung bestand. Im Juli 2001 legte ich die Diplom-Chemiker-Hauptprüfung ab.

In der Zeit von August 2001 bis April 2002 fertigte ich im Arbeitskreis von Prof. Dr. G. Krausch an der Universität Bayreuth meine Diplomarbeit mit dem Titel „AFMUntersuchung an Biopolymeren“ an.

Seit Juni 2002 arbeite ich unter Anleitung von Prof. Dr. H. Laatsch an der vorliegenden Dissertation.

Ich besitze die indonesische Staatsangehörigkeit. 